\title{
Cooling and Internal Waves on the Continental Shelf
}

by

\section{James Maxwell Pringle}

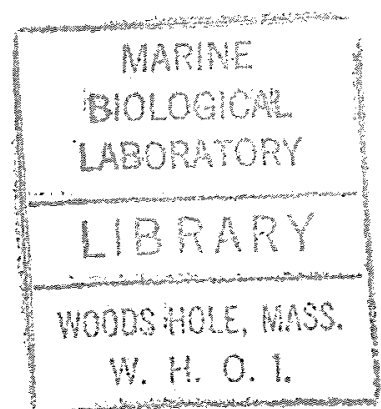

B.A. Dartmouth College, (1990)

Submitted in partial fulfillment of the requirements for the degree of

Doctor of Philosophy

at the

\section{MASSACHUSETTS INSTITUTE OF TECHNOLOGY \\ and the}

\section{WOODS HOLE OCEANOGRAPHIC INSTITUTION}

June 1998

(C) James Maxwell Pringle 1998

The author hereby grants to MIT and to WHOI permission to reproduce and to distribute copies of this thesis document in whole or in part.

Signature of Author .................

Joint Program in Physical Oceanography Massachusetts Institute of Technology

Woods Hole Oceanographic Institution May 1, 1998

Certified by $\ldots \ldots \ldots \ldots \ldots \ldots \ldots \ldots \ldots \ldots \ldots \ldots$

Kenneth H. Brink Senior Scientist Thesis Supervisor

Accepted by

W. Brechner Owens

Chairman, Joint Committee for Physical Oceanography Massachusetts Institute of Technology Woods Hole Oceanographic Institution 


\title{
Cooling and Internal waves on the Continental Shelf
} by

James Maxwell Pringle

Submitted in partial fulfillment of the requirements for the degree of Doctor of Philosophy at the Massachusetts Institute of Technology and the Woods Hole Oceanographic Institution

April 29, 1998

\begin{abstract}
In order to begin to understand the behavior of an ice-free coastal ocean exposed to winter time cooling, the evolution of an initially homogeneous body of water undergoing uniform surface heat loss and wind forcing is examined. The dynamics of this model ocean are modulated by the intense vertical mixing driven by the surface cooling, which in the initially homogeneous ocean is sufficient to mix the entire water column in less than an inertial period. This strong vertical mixing prevents the formation of geostrophic flows and so flows are nearly down pressure gradient and downwind. Cross-shelf temperature gradients are formed by uniform cooling over water of differing depths, and these temperature gradients lead to crossshelf density gradients. The cross-shelf density gradients and flows can prevent the cooling-driven vertical mixing from reaching the bottom when the cross-shelf bottom velocity exceeds

$$
v_{c}=-\frac{Q}{h}\left(\frac{\partial \rho}{\partial y}\right)^{-1}
$$

(where $h$ is the water depth, $Q$ is the surface density flux representing cooling, and $\partial \rho / \partial y$ is the cross-shelf density gradient).

Once the surface cooling is prevented from mixing the entire water column, alongshore geostrophic flows form, and these flows can be baroclinically unstable. The unstable flow quickly can achieve a steady state in which the length scale of the eddies is governed by either the Rhines arrest scale or a frictional arrest scale, and the surface heat flux is balanced by a cross-shelf heat flux driven by the eddies. Scales are found for the cross-shelf density gradient which results from this balance, and these scales are tested successfully in a numerical model. It is found with the numerical model that the balance between surface cooling and the cross-shelf heat flux can be attained in less than a winter with conditions typical of the Mid-Atlantic Bight.
\end{abstract}

Linear internal waves shoal and are dissipated as they cross the continental shelf, and, in order to understand how this affects the internal wave climate on the shelf, internal wave solutions are found for a wedge-shaped bathymetry. These 
solutions for linear internal waves in the presence of linear bottom friction and barotropic alongshore mean flows are approximate; they are based on flat-bottom vertical modes, and the horizontal propagation is found by ray-tracing. It is found that bottom friction is capable of entirely dissipating the waves before they reach the coast, that waves traveling obliquely offshore are reflected back to the coast from an offshore caustic, and that without a mean current, the maximum distance an internal wave ray can travel along the coast is twice the distance from the shore to the offshore caustic.

The solutions for internal waves propagating over a wedge-shaped bathymetry are used to predict the evolution of an ensemble of internal waves whose properties match those of a Garrett and Munk internal wave spectrum at a point offshore. This is meant to be a simple model of the evolution of an oceanic internal wave spectrum across a continental shelf. The shape of the current ellipse caused by this ensemble of internal waves at a given frequency is found to be largely independent of frequency. It is also found that the orientation of the current ellipse is controlled by the alongshore mean currents and the "redness" of the oceanic internal wave spectrum. Because of friction, it is found that internal waves generated in the deep ocean are more likely to be important to the internal wave climate of narrow shelves than wide shelves.

The internal wave climate near two moorings of the Coastal Ocean Dynamics Experiment observation program is analyzed. It is found that the high frequency internal wave energy levels are elevated above the Garrett and Munk spectrum, and the spectrum becomes less red as one moves towards shore. The shape of the current ellipses is largely independent of frequency in the internal wave band, and the major axis is approximately perpendicular to the isobaths. The wave field is dominated by vertical-mode one waves. It is concluded that the internal wave energy is predominantly moving towards the shore from the shelf break, but that a significant portion of the internal wave energy over the continental shelf is generated over the continental shelf.

Thesis Supervisor: Kenneth H. Brink

Senior Scientist, Department of Physical Oceanography

Woods Hole Oceanographic Institution 


\section{Acknowledgments}

First and foremost, I would like to thank Mom, Dad, and Annie, for setting me down this path, and Ken Brink for giving me the advice I needed to stay on it until I had this thesis.

My friends kept me sane. The Dartmouth crowd, all the Jens, all the friends in the Joint Program, they all did their part. Perhaps I would have graduated more quickly without their steady distractions, but it would not have been a life I could have lived. (I would not have played as much tennis, met with a bear in a half-frozen New Hampshire swamp, gone camping in $-25^{\circ} \mathrm{F}$ weather (Amara!), drunk vodka in Nizhny Novgorod (or fixed Russian showers in Moscow), snowboarded, grilled nearly as much, or played the part of an Austrian Duke on a train traveling incognito while suspected of a contrived plot (thanks Casa del Angst)).

My class in physical oceanography: Jay, Lyn, Bill, Natalia, François, Derek, Stefan, Brian, \& Miles, from whom I learned much of what I know about oceanography and fellowship under stress.

My officemates and housemates, Clayton, Ed, Jay, Susan, Kelsey, Alison, Gwyneth, Jim, Sarah \& Steve; they seem to have survived intact.

And, of course, my committee who, with great fortitude, read way to many drafts and still gave me good comments. Without Paola Rizzoli, Steve Lentz, John Trowbridge, Glen Gawarkiewicz, and Bob Beardsley, my thesis would have been much worse.

This work was funded by an Office of Naval Research fellowship and and Office of Naval Research AASERT fellowship, N00014-95-1-0746. 


\section{Contents}

1 Introduction $\mathbf{9}$

1.1 Winter Time Cooling and the Coastal Ocean . . . . . . . . . . . . 9

1.2 Observations and Theory of High Frequency Internal Waves over the Continental Shelf . . . . . . . . . . . . . . . 12

1.3 Bibliography........................... 14

2 The Effect of Surface Cooling-Driven Vertical Mixing on Larger Scale Coastal Dynamics, and Vice Versa. 17

2.1 Introduction . . . . . . . . . . . . . . . . . . 18

2.2 The Parameterization of Convection . . . . . . . . . . . . . . 20

2.3 Flow Fields When Convection Reaches the Bottom . . . . . . . . . . 22

2.4 When Does Convection Not Mix the Entire Water Column? . . . . . 27

2.5 When Convection no Longer Mixes to the Bottom . . . . . . . . . . . 41

2.6 When Cooling Falters . . . . . . . . . . . . . . . . 45

2.7 Conclusions . . . . . . . . . . . . . . . . . . . . . . . . . . . 45

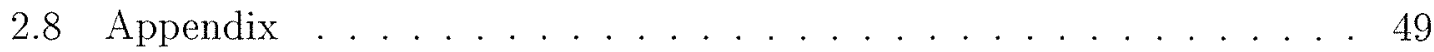

2.9 Bibliography . . . . . . . . . . . . . . . . . 53

3 The Role of Cross-Shelf Eddy Transport in the Steady State Response of a Wind-Free Coastal Ocean to Winter Time Cooling 55

3.1 Introduction . . . . . . . . . . . . . . . . 57

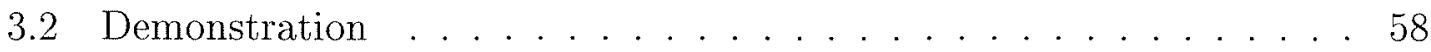

3.3 Outline of the Scaling for the Steady State $\partial \bar{\rho} / \partial y \ldots \ldots$. . . . . . 64

3.4 Buoyancy/Heat Balance . . . . . . . . . . . . . . 65

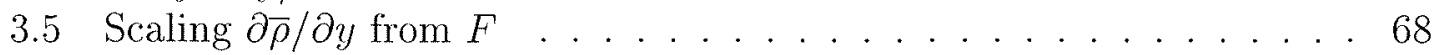

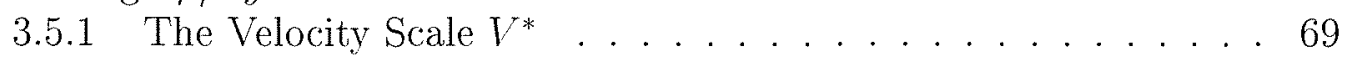

3.5.2 The Correlation Between $v$ and $\rho: \gamma \ldots \ldots . . . . . . . .69$

3.5.3 The Cross-Shelf Length Scale: $L^{*} \ldots . . . . . . . . . .770$

3.5.4 Given $V^{*}$ and $L^{*}$, what are $F, \overline{\rho_{y}}$ and $\mathcal{L}$ ? . . . . . . . . . 73

3.6 Testing the Scales in a Numerical Model . . . . . . . . . . . . . . 74

3.6.1 Flat Bottom Results . . . . . . . . . . . . . . . 74

3.6.2 The Transition Between $\mathcal{L}<1$ to $\mathcal{L}>1 \ldots . . . . .81$ 
3.6.3 Testing the Length and Velocity Scales . . . . . . . . . 8 86

3.6.4 Where the Scaling Breaks Down . . . . . . . . . . . . . 90

3.7 Can the Scalings Above be Used to Understand The Transient Behavior of the Density Field? . . . . . . . . . . . . . . . . . 93

3.8 Extending the Scalings to Passive Tracers . . . . . . . . . 107

3.9 How do the Cross-Shelf Eddy Heat Fluxes Compare With The Wind Driven Heat Fluxes? . . . . . . . . . . . . . . . . . . . . 111

3.10 Conclusion . . . . . . . . . . . . . . . . . . . . . . . 112

3.11 Appendix . . . . . . . . . . . . . . . . 114

3.12 Bibliography . . . . . . . . . . . . . . 117

4 High Frequency Linear Internal Waves on a Sloping Shelf 121

4.1 Introduction . . . . . . . . . . . . . . . . . 122

4.2 Plain Internal Wave Solution . . . . . . . . . . . . . . . 123

4.3 The Path of a Wave with No Mean Flow . . . . . . . . . . . . . 127

4.4 Wave Amplitude: the Inviscid Problem . . . . . . . . . . . . . . 130

4.5 Wave Amplitude: the Frictional Problem . . . . . . . . . . . . . . 133

4.6 Internal Waves in the Presence of a Barotropic Mean Flow . . . . . 140

4.7 Current Meter Observations- The No Mean Flow Case . . . . . . 147

4.8 Current Meter Observations- The Mean Flow Case . . . . . . . . . 152

4.9 Conclusions . . . . . . . . . . . . . . . . . . . . . . 158

4.10 Appendix A: The Internal Wave Bottom Boundary Condition . . . 162

4.11 Appendix B: The Caustic at $x_{c} \ldots \ldots \ldots \ldots \ldots$

4.12 Bibliography . . . . . . . . . . . . . . . . . . . 173

5 Observations of High Frequency Internal Waves in the CODE Region 175

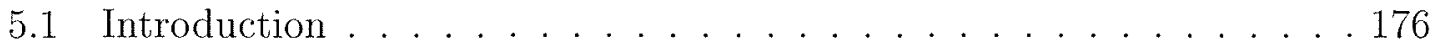

5.2 Internal Wave Background . . . . . . . . . . . 177

5.3 Topography and Coordinates . . . . . . . . . . . . . 178

5.4 Hydrography . . . . . . . . . . . . . . . . . . . . . . . 180

5.5 Low Frequency Currents . . . . . . . . . . . . . . . . . 184

5.6 Analysis at individual Current Meters . . . . . . . . . . . . . 184

5.6 .1 Power . . . . . . . . . . . . . . . . . . . 186

5.6 .2 Spectral Shape . . . . . . . . . . . . . . . . 186

5.6.3 The Lack of Isotropy- Predictions from PB . . . . . . . . . 189

5.6 .4 Ellipticity . . . . . . . . . . . . . . . . . . . . 192

5.6 .5 Current Ellipse Orientation . . . . . . . . . . . 196

5.7 Modal Decomposition . . . . . . . . . . . . . . . . . . . . 199

5.8 Variation in Power with Space and Time . . . . . . . . . 208

5.9 Discussion and Conclusion . . . . . . . . . . . . . . . . . 210

5.10 Acknowledgments . . . . . . . . . . . . . . . . . 211

5.11 Appendix: The Modal Decomposition . . . . . . . . . . . . . 212

5.12 Bibliography . . . . . . . . . . . . . . . . . . 217 
6 Conclusion $\quad 219$

6.1 Winter Time Cooling and the Coastal Ocean . . . . . . . . . . . . . . 219

6.2 Observations and Theory of High Frequency Internal Waves over the

Continental Shelf . . . . . . . . . . . . . . . . . 222

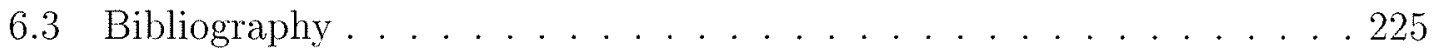




\section{Chapter 1}

\section{Introduction}

\subsection{Winter Time Cooling and the Coastal Ocean}

A hydrographic section taken off of Nantucket Shoals at the end of the winter, figure 1.1, shows cold dense water near the coast which becomes warmer and less dense offshore, until the shelf break front [Beardsley et al., 1985]. The isopyncnals have sufficient slope to intersect the surface and the bottom, satisfying a necessary but not sufficient criterion for baroclinic instability [Pedlosky, 1987].

Chapters two and three present a simple model of the origin of this hydrography inshore of the shelf break front during the winter. Along the east coast of the United States, the winter differs from the summer by being stormier and colder. The simple model concentrates on the effect of cooling and largely neglects the effects of winds on the coastal ocean.

Surface cooling makes the water at the surface denser. This drives convection, and this convection can mix the water column vertically. The cooling can be strong enough to mix the water column in less than an inertial period, erasing vertical density gradients and transporting momentum from the surface to the bottom boundary layer. When the momentum is mixed through the water column in less than an inertial 

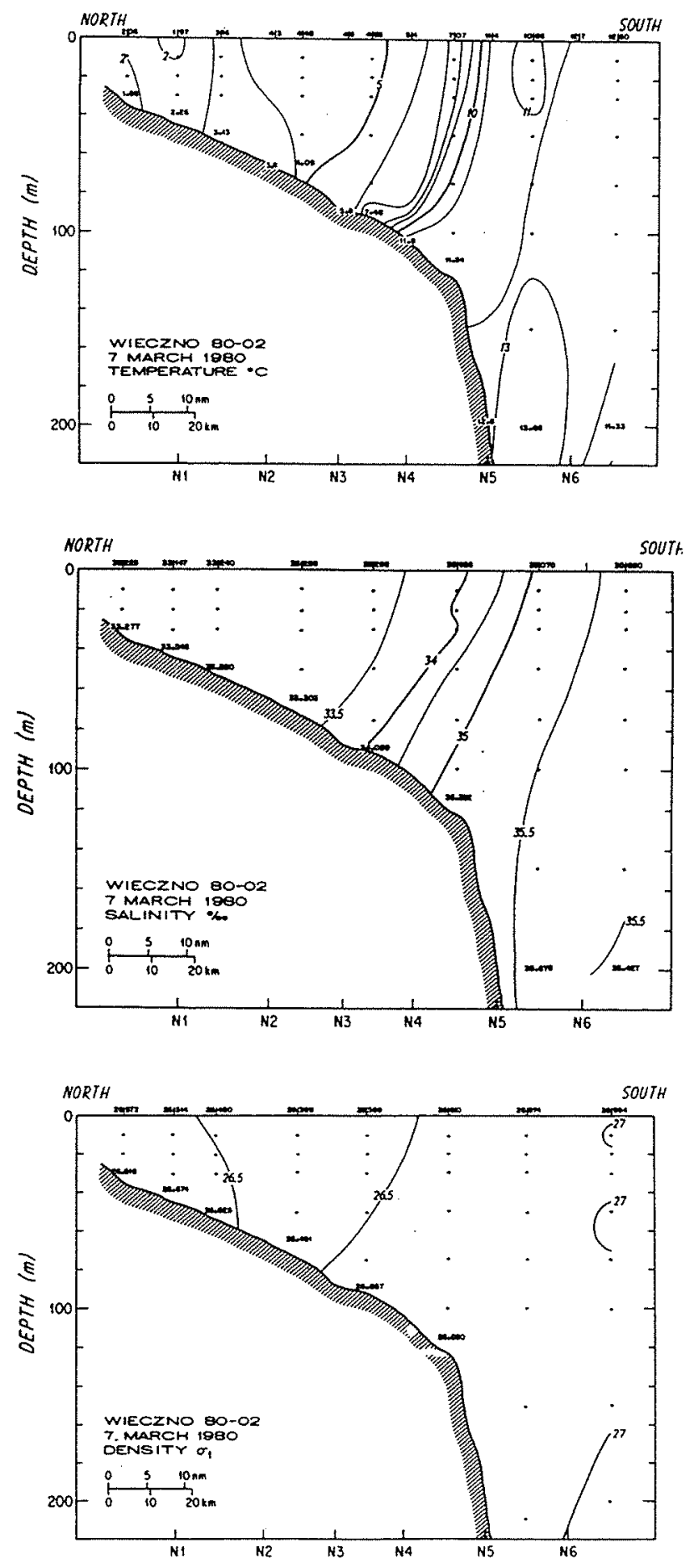

Figure 1.1: The temperature, salinity, and potential density of a hydrographic section of the Middle Atlantic Bight taken in march of 1980 as part of Nantucket Shoals Flux Experiment (NSFE79). Defd as: fig-nsfe 
period, the water becomes part of the inner shelf, where merged bottom and surface boundary layers reduce the role of rotation. This is a very different dynamical regime from that which normally obtains over the shelf, and it is thus important to discover when the winter time ocean is caused by cooling to be part of the inner shelf [Lentz, 1995]. The second chapter discusses when the cooling is capable of mixing the entire water column in less than an inertial period, and how this effects the dynamics.

If there is no ice to insulate the water, the length scales of atmospheric cooling are likely to be set by the large scales of the atmospheric weather systems. If heat is not transported efficiently across the shelf by the ocean circulation, this large scale atmospheric cooling will make the shallower waters colder than the deeper waters. This cross-shelf temperature gradient will produce a cross-shelf density gradient with denser water near the shore. When convectively driven turbulence mixes to the bottom it can suppress the cross-shelf transport of heat, allowing the cross-shelf density gradients to grow stronger as the cooling continues [Nunes Vas, Lennon and de Silva Samarasinghe, 1989]. Once the cooling is prevented from mixing straight, to the bottom and the effects of rotation dominate the flow, the cross-shelf density gradients mainly drive alongshelf geostrophic flows. In either case, the cross-shelf flows driven by the cross-shelf density gradients are ageostrophic, small, and incapable of driving a significant cross-shelf heat flux. The cross-shelf density gradients, however, have the potential to be baroclinically unstable. In the mid-latitudes of the atmosphere, baroclinic instabilities driven by meridional density gradients drive meridional heat fluxes which balance the solar heating [Green, 1970; Stone, 1972]. These baroclinic instabilities in the atmosphere are also important in transporting tracers meridionally in the atmosphere [James, 1994]. Motivated by these atmospheric dynamics, and by the related oceanic work of Visbeck, Marshall and Jones [1996] and Chapman and Gawarkiewicz [1997], chapter 3 examines a balance between surface cooling and the cross-shelf heat flux driven by instabilities in the flow field. 
The transport of passive tracers (nutrients, pollution, etc.) by these instabilities are also examined.

\subsection{Observations and Theory of High Frequency Internal Waves over the Continental Shelf}

Non-barotropic variability over a stratified continental shelf in the frequency band between the inertial frequency and the buoyancy frequency will likely be dominated by internal wave dynamics, and so a first step to understanding this variability is to understand the dynamics of linear internal waves over a sloping bottom.

One source of this high frequency energy is the deep ocean. It is estimated that $10 \%$ of all tidal energy is dissipated by internal waves impinging on the shelf break [Hendershott, 1981 p. 337]. The fate of this internal wave energy after it crosses the shelf break is not well understood. Some predict it will be dissipated by wave breaking [Gordon, 1978], while others suggest bottom friction [Brink, 1988].

This energy flux carried from the deep ocean by the internal waves can affect the coastal ocean. The internal wave energy flux onto the shelf, if it were used entirely to mix away the stratification, would do so relatively rapidly. It can be shown that the energy flux onto the shelf given by assuming a Garrett and Munk [1972] spectrum at the shelf break is capable of mixing a typical west-coast shelf in $\mathrm{O}(10$ days $)$. This calculation could be very misleading, however, because it is unclear how much of the internal wave energy dissipated by bottom friction and other mechanisms is available to mix stratified water.

Understanding the evolution of internal waves as they propagate across the shelf is also important to understanding observations of internal waves on the shelf. If one were to observe the horizontal wave number spectrum of an internal wave field, one could trace the waves back past where they were generated. If one has only a 
horizontally incoherent array of current meters, it is impossible to find the horizontal wavenumber spectrum of the waves, and one way to begin to understand the sources and sinks of the internal waves is to hypothesize a source and calculate how the internal wave spectrum evolves across the shelf. This can then be compared to the observations.

Chapter 4 takes three steps towards understanding the evolution of the high frequency internal wave spectrum across the shelf. First, the evolution of a single linear internal wave over a sloping bottom in an inviscid ocean is derived. A solution to this was found by McKee [1973]; however, that solution, while exact, involves Bessel functions of high imaginary orders, making it difficult to evaluate and difficult to extend to differing bottom geometries. The solution in chapter 4 is an approximate one found by assuming that the vertical structure of the waves is given by flat bottom vertical modes and using ray tracing to solve for the horizontal propagation of the waves. While this solution is only valid for waves whose frequency is higher than the frequency for critical reflection from the bottom, the solution is considerably easier to manipulate than the exact solutions. The solutions are then extended to include bottom friction and mean barotropic alongshore flows.

The solutions for individual internal waves are then used to derive the evolution of the internal wave spectra across the shelf given the assumption that a Garrett and Munk [1972] spectrum impinges on the shelf break.

In chapter 5 these results are used to help interpret observations of internal waves taken as part of the CODE II experiment on the California continental shelf. It is found that internal wave energy was propagating in from the ocean to the coast and being dissipated along the way, but that an appreciable fraction of the internal wave energy over the continental shelf was likely to have been generated over the continental shelf. 


\subsection{Bibliography}

Beardsley, R. C., D. C. Chapman, K. H. Brink, S. R. Ramp and R. Schlitz, The Nantucket Shoals Flux Experiment (NSFE79). Part I: A Basic Description of the Current and Temperature Variability, J. Phys. Oceanogr., 15(6), 713-748, 1985.

Brink, K. H., On the effect of bottom friction on internal waves, Continental Shelf Research, 8(4), 397-403, 1988.

Chapman, D. C. and G. Gawarkiewicz, Shallow Convection and Buoyancy Equilibration in an Idealized Coastal Polynya , J. Phys. Oceanogr., 27(4), 555-566, 1997.

Garrett, C. and W. Munk, Space-Time Scales of Internal Waves, Geophys. Fluid Dyn., $3,225-264,1972$.

Gordon, R. L., Internal wave climate near the coast of northwest Africa during JOINT-1, Deep Sea Res., 25, 625-643, 1978.

Green, J. S. A., Transfer Properties of the Large-Scale Eddies and the General Circulation of the Atmosphere, Quart. J. R. Met. Soc., 96(408), 157-185, 1970.

Hendershott, M. C., Long Waves and Ocean Tides, in Evolution of Physical Oceanography, edited by B. A. Warren and C. Wunsch, pp. 292-339, Massachusetts Institute of Technology, 1981.

James, I. N., Introduction to Circulating Atmospheres, Cambridge University Press, New York, 1994.

Lentz, S. J., Sensitivity of the Inner-Shelf Circulation to the Eddy Viscosity Profile, J. Phys. Oceanogr., 25, 19-28, 1995.

McKee, W. D., Internal-inertial Waves in a Fluid of Variable Depth, Proc. Camb. Phil. Soc., 73, 205-213, 1973. 
Nunes Vas, R. A., G. W. Lennon and J. R. de Silva Samarasinghe, The Negative role of Turbulence in Estuarine Mass Transport, Estuarine, Coastal and Shelf Science, 28, 361-377, 1989.

Pedlosky, J., Geophysical Fluid Dynamics (second edition), Springer-Verlag, New York, 1987.

Stone, P. H., A Simplified Radiative-Dynamical Model for the Static Stability of Rotating Atmospheres, J. Atmos. Sci., 29(3), 405-418, 1972.

Visbeck, M., J. Marshall and H. Jones, Dynamics of Isolated convective Regions in the Ocean, J. Phys. Oceanogr., 26, 1721-1734, 1996. 


\title{
Chapter 2
}

\section{The Effect of Surface}

\section{Cooling-Driven Vertical Mixing on Larger Scale Coastal Dynamics,}

\section{and Vice Versa.}

\begin{abstract}
A coastal ocean undergoing spatially-uniform winter-time cooling will become colder in the shallows near the shore than in the deeper waters as the same heat loss is distributed over less depth. This cross-shelf temperature gradient can cause a crossshelf density gradient which can drive a circulation over the shelf. This circulation can be in one of two differing regimes, depending on whether the vertical mixing caused by cooling-driven convection mixes the entire water column in less than an inertial period, or not. The cross-shelf circulation can prevent convection from mixing the entire water column by advectively restratifying the water near the bottom.

The two dynamical regimes are studied, and a criterion for the transition between them is found. The criterion is tested in a primitive equation model. Oscillations between the two regimes and dependencies on the history of the forcing are found and explained.
\end{abstract}




\section{$2.1 \quad$ Introduction}

In the more northern latitudes, coastal waters are subject to strong cooling for much of the winter. In the absence of ice, the spatial scale for the surface heat flux is set by the large spatial scales of the atmosphere. The effect of the cooling on the coastal waters is governed by the depth of the water (for shallower waters will become colder than deeper waters for a given heat loss), and by the circulation of the water which can transport heat across the shelf.

The circulation that transports heat across the shelf is driven by the wind and the cross-shelf density gradients formed when shallower nearshore waters become cooler than deeper offshore waters. The circulation is modified by the vertical mixing driven by cooling and two regimes can be identified: one where vertical mixing dominates the solution and the Earth's rotation little affects the flow, and another where vertical mixing is suppressed by the cross-shelf circulation so that the Earth's rotation strongly affects the flow. This chapter describes the dynamics of these two regimes and the transition between them.

Previous works examining cooling of a coastal ocean have not specifically considered the effect of cooling-driven vertical mixing. In Chapman and Gawarkiewicz [1997] and Chapman [1998], cooling-driven convective mixing was not examined because the cooling was only applied to a limited portion of the domain and the circulation examined was primarily outside of the cooling region. Condie and Rhines [1994] apply cooling over the entire domain, but assume a priori that the cross-shelf flow is capable of preventing convection from mixing to the bottom in all but a small portion of the domain. They run their laboratory experiments until this true.

The simplest model of the response of shelf waters to cooling is a wedge shaped bathymetry with a surface heat loss that is uniform everywhere (figure 2.1). The forcing, geometry and flow are assumed not to vary in the alongshore direction. This model will be used to address the following questions: 


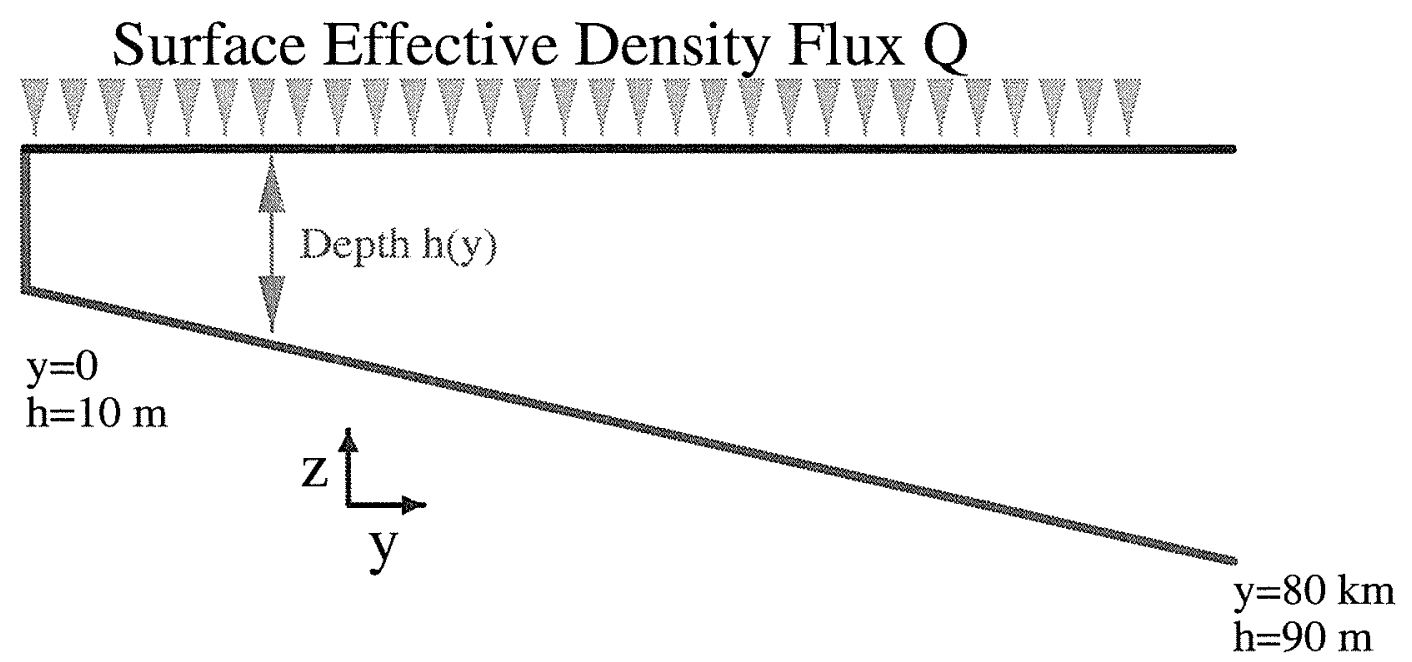

Figure 2.1: The geometry and forcing of the model considered here.

- What are the bulk effects of convection on the flow field, e.g. how can one represent the overall effect of many small convection cells?

- What does the flow field look like when the vertical mixing driven by cooling dominates the solution?

- When does the circulation prevent the cooling-driven mixing from mixing the entire water column?

- How does the circulation change when the cooling-driven mixing is prevented from mixing the entire water column?

These questions will be addressed both analytically and numerically. The numerical model used is SPEM 5.1, a rigid lid, Boussinesq, finite difference primitive equation model whose adaptation to this problem is described in the appendix. The equation of state for both the analytic and numerical work is linear, which allows the heat flux to be represented as a density flux. The geometry and cooling are chosen to be representative of the Mid-Atlantic Bight, which has a gentle bottom slope of $\mathrm{O}\left(10^{-3}\right)$ and experiences winter time cooling rates of $\mathrm{O}\left(200 \mathrm{~W} \mathrm{~m}^{-2}\right)[$ Brown 
and Beardsley, 1978; Mountain, Stout and Beardsley, 1996]. The cooling will be represented as a density flux, $Q$, of $7 \times 10^{-6} \mathrm{~kg} \mathrm{~m}^{-2} \mathrm{~s}^{-1}$, which is equivalent to a heat loss of $170 \mathrm{~W} \mathrm{~m}^{-2}$ out of $10^{\circ} \mathrm{C}$ water or $300 \mathrm{~W} \mathrm{~m}^{-2}$ out of $3^{\circ} \mathrm{C}$ water.

\subsection{The Parameterization of Convection}

The dynamics of convection are non-hydrostatic and occur on horizontal scales of the same order as the water depth [Emanuel, 1994]. This work parameterizes the effects of convection in order to focus on the larger scale flow. The parameterization below gives vertical diffusivities of the same order as Mellor and Yamada [1982] and is used instead of Mellor and. Yamada [1982] for simplicity.

A diffusive parameterization of convection implies eddy diffusivities which scale as the product of a convective length scale and a convective velocity scale. A reasonable length scale for the vertical mixing caused by convection on the shelf is the water depth. Given a buoyancy flux at the surface of $g Q / \rho_{0}$ and the depth $h$, the only parameter with units of velocity one can assemble is

$$
w^{*}=\left[-\frac{g h}{\rho_{0}} Q\right]^{\frac{1}{3}} .
$$

In both atmospheric boundary layer experiments [Mellor and Yamada, 1982; Stull, 1988] and laboratory experiments with water [Fernando, Chen and Boyer, 1991; Maxworthy and Narimousa, 1994], this scale has proven to be a good one for the vertical velocity of a non-rotationally controlled convective region.

Using $w^{*}$ as a velocity scale and the water depth $h$ as a length scale, a diffusivity for heat, $\nu$, and momentum, $A$, can be scaled as $A=\nu=O\left(w^{*} h\right)$. The constant of proportionality relating $w^{*} h$ to $A$ and $\nu$ will be assumed to be 0.25 hereafter in order to agree better with measurements of $w$ presented in Mellor and Yamada [1982] and Stull [1988] (They observe turbulent convective velocities of about $0.5 w^{*}$, and if the 


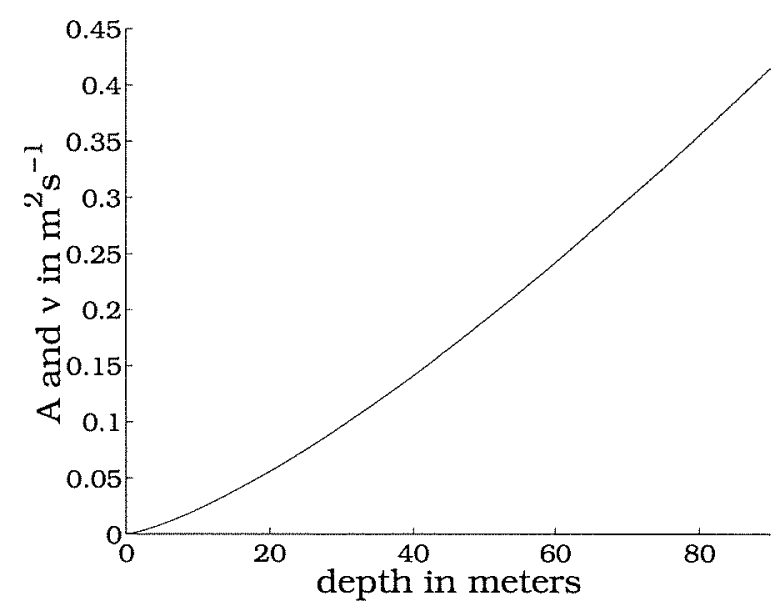

Figure 2.2: The mass diffusivity and momentum viscosity as a function of water depth during unstable stratification for $Q=7 \times 10^{-6}$.

mixing length is about the distance from a boundary, $0.5 h$ is a good length scale.)

$$
A=\nu=\frac{1}{4} w^{*} h=\frac{1}{4} h^{\frac{4}{3}}\left[\frac{g}{\rho_{0}} Q\right]^{\frac{1}{3}} .
$$

A density flux of $Q=7 \times 10^{-6} \mathrm{~kg} \mathrm{~m}^{-2} \mathrm{~s}^{-1}$ gives the diffusivities shown in figure 2.2.

The generation of turbulence by the wind has been neglected even though it would be expected to increase the turbulent diffusivities. A $0.1 \mathrm{~N} \mathrm{~m}^{-2}$ wind stress produces a turbulent velocity $u^{*}$ of

$$
u^{*}=\sqrt{\frac{\tau}{\rho_{0}}}=10^{-2} \mathrm{~ms}^{-1} .
$$

The free convective velocities $w^{*}$ from $(2.1)$ range from $10^{-2} \mathrm{~m} \mathrm{~s}^{-1}$ in 20 meters of water to $2 \times 10^{-2} \mathrm{~m} \mathrm{~s}^{-1}$ in 100 meters of water for the cooling used in this paper, and so are of the same order as $u^{*}$. It is unclear in the literature how the additional turbulence from the wind would be distributed through the water column, but if it 
worked over the entire water column it could as much as double the eddy diffusivity. Since the scaling for $\nu$ and $A$ is only meant to be good to an $\mathrm{O}(1)$ constant, the effect of wind-generated turbulence is less than the uncertainty of the convective diffusivity.

It is important to determine when rotation affects convection. Rotation ought not to affect convection when the time scale of convection, $2 h w^{*-1}$, is less than an inertial period, $2 \pi f^{-1} .2 h w^{*-1}$ is less than $2 \pi f^{-1}$ when

$$
h<\left(\frac{\pi}{2}\right)^{\frac{3}{2}} \sqrt{\frac{g Q}{\rho_{0} f^{3}}}
$$

Experiments bear out this depth limit, though the constant before (2.4) is often a value other than $(0.5 \pi)^{\frac{3}{2}}$ (about 2). Maxworthy and Narimousa [1994] find that the constant should be 12.7, and Fernando, Chen and Boyer [1991] in their experiments find a value of 4.5 .

This length scale is 700 meters for a mass flux of $Q=7 \times 10^{-6} \mathrm{~kg} \mathrm{~m}^{-2} \mathrm{~s}^{-1}$. Thus a convection cell in the the 10 to 100 meter ocean that is examined here will not feel the Earth's rotation.

\subsection{Flow Fields When Convection Reaches the Bottom}

When surface cooling is started in the homogeneous fluid of density $\rho_{0}$, convection quickly extends to the bottom. The fluid then has very high vertical mixing coefficients, and thus the two dimensional flow field is unable to transport significant heat across the shelf, and the heat balance is one dimensional (this will be justified in section 2.4). The density field forced by a surface density flux $Q$ is thus 
approximately

$$
\rho=\frac{Q t}{h}+\rho_{0}
$$

and the cross-shelf density gradient is

$$
\frac{\partial \rho}{\partial y}=-\frac{Q t}{h^{2}} \frac{\partial h}{\partial y}
$$

where $t$ is the amount of time the cooling has been active and $y$ is the cross-shelf direction. The time scale of the change in this density gradient is the time since cooling began, and the time it takes for the momentum dynamics to adjust to the density gradient is the much smaller diffusion time scale $h^{2} A^{-1}$ (of order hours). The momentum equations can thus be solved as if they were steady. The nonlinear terms can be neglected because of the large spatial scale of the forcing. The equations of motion

$$
\begin{aligned}
\frac{\partial u}{\partial t}+\vec{u} \nabla u-f v & =\frac{\partial}{\partial z}\left(A \frac{\partial u}{\partial z}\right), \\
\frac{\partial v}{\partial t}+\vec{u} \nabla v+f u & =-\frac{1}{\rho_{0}} \frac{\partial P}{\partial y}+\frac{\partial}{\partial z}\left(A \frac{\partial v}{\partial z}\right), \\
P & =P_{0}+\int_{z}^{0} g \rho d z, \\
v_{y}+w_{z} & =0,
\end{aligned}
$$

can then be reduced to

$$
\begin{aligned}
f v & =\frac{\partial}{\partial z}\left(A \frac{\partial u}{\partial z}\right) \\
f u & =-\frac{1}{\rho_{0}} \frac{\partial P}{\partial y}+\frac{\partial}{\partial z}\left(A \frac{\partial v}{\partial z}\right) \\
P & =P_{0}+g \frac{\partial \rho}{\partial y} z
\end{aligned}
$$


where $P_{0}$ is the surface pressure.

The ratio of the Ekman depth, $\sqrt{2 A / f}$, to the water depth varies from 4 to 1.4 for the viscosities given in (2.2) and the cooling rate used here of $7 \times 10^{-6}$ $\mathrm{kg} \mathrm{m}^{-2} \mathrm{~s}^{-1}$. Since the Ekman depth is greater than the water depth, it would seem that rotation could be ignored. However, if the forcing in one direction is greater than the forcing in the other direction this may not be true. This can be seen by scaling the equations with (where primes denote non-dimensional variables)

$$
\begin{aligned}
& z=h z^{\prime} \\
& u=U\left(u_{0}^{\prime}+2 \frac{h^{2}}{\delta_{E}^{2}} u_{1}^{\prime}\right), \\
& v=V\left(v_{0}^{\prime}+2 \frac{h^{2}}{\delta_{E}^{2}} v_{1}^{\prime}\right),
\end{aligned}
$$

where $\delta_{E}$ is the Ekman depth, $U$ is the scale alongshelf velocity and $V$ the scale crossshelf velocity. The parameter $h^{2} / \delta_{E}^{2}$ is assumed small, though when the results are compared to full solutions, they are found to be reasonable even for $h^{2} / \delta_{E}^{2} \approx 1$. When the alongshelf and cross-shelf forcing are of the same order, and $U=V$, the equations can then be written in a non-dimensional form as

$$
\begin{aligned}
0 & =\frac{\partial^{2} u_{0}^{\prime}}{\partial z^{\prime 2}} \\
0 & =-\frac{h^{2}}{V A \rho_{0}} \frac{\partial P_{0}^{0}}{\partial y}-\frac{g h^{3}}{V A} \frac{\partial \rho}{\partial y} z^{\prime}+\frac{\partial^{2} v_{0}^{\prime}}{\partial z^{\prime 2}}
\end{aligned}
$$

to first order and with

$$
\begin{aligned}
& \frac{V}{U} v_{0}^{\prime}=\frac{\partial^{2} u_{1}^{\prime}}{\partial z^{\prime 2}} \\
& \frac{U}{V} u_{0}^{\prime}=-\frac{h^{2}}{V A \rho_{0}} \frac{\partial P_{0}^{1}}{\partial y}+\frac{\partial^{2} v_{1}^{\prime}}{\partial z^{\prime 2}}
\end{aligned}
$$

as a second order correction. $\left(P_{0}^{0}\right.$ and $P_{0}^{1}$ are surface pressure gradients associated with the first and second order solutions, and are not part of the forcing. They 
will be found as part of the solution.) The boundary conditions for the first order equations are:

$$
\begin{aligned}
\frac{\partial u_{0}^{\prime}}{\partial z} & =\frac{\tau_{\text {wind }}^{x} h}{U A \rho_{0}} @ z=0 \\
\frac{\partial u_{0}^{\prime}}{\partial z} & =\frac{r h}{A} u_{0}^{\prime} @ z=-h \\
\frac{\partial v_{0}^{\prime}}{\partial z} & =\frac{\tau_{\text {wind }}^{y} h}{V A \rho_{0}} @ z=0 \\
\frac{\partial v_{0}^{\prime}}{\partial z} & =\frac{r h}{A} v_{0}^{\prime} @ z=-h \\
0 & =\int_{-h}^{0} v_{0}^{\prime} d z,
\end{aligned}
$$

and for the second order equations the boundary conditions are:

$$
\begin{aligned}
\frac{\partial u_{1}^{\prime}}{\partial z} & =0 @ z=0 \\
\frac{\partial u_{1}^{\prime}}{\partial z} & =\frac{r h}{A} u_{1}^{\prime} @ z=-h \\
\frac{\partial v_{1}^{\prime}}{\partial z} & =0 @ z=0 \\
\frac{\partial v_{1}^{\prime}}{\partial z} & =\frac{r h}{A} v_{1}^{\prime} @ z=-h \\
0 & =\int_{-h}^{0} v_{1}^{\prime} d z .
\end{aligned}
$$

The depth-integral of the cross-shelf velocity is zero by the combination of (2.7d) and no flow through the coast, and $\tau_{\text {wind }}^{x}$ and $\tau_{\text {wind }}^{y}$ are the along- and cross-shelf wind stress respectively. The bottom boundary condition is a linear drag law. Because the windstress and density gradient forcing enter only in the first order equations, the non-dimensionalization indicates that the cross-shelf velocity will not be affected by rotation except for a correction of order $h^{2} \delta_{E}^{-2}$ of the alongshelf velocity, and similarly the alongshelf velocity will only be affected by rotation to an amount which scales as $h^{2} \delta_{E}^{-2}$ the cross-shelf velocity.

When the forcing is predominantly in the alongshelf direction and $U$ is much 
greater than $V,(2.10 \mathrm{~b})$ and $(2.11 \mathrm{~b})$ are no longer consistent because $(U / V) *\left(h^{2} / \delta_{E}^{2}\right)$ will be order one. However, the solution to (2.10b) and (2.11b) can be summed in order to find a solution valid to within an error of $h^{4} / \delta_{E}^{4} u_{0}$. In this case the sum of the solutions to (2.10b) and (2.11b) represent the first order solution to the cross-shelf momentum balance, and implies cross-shelf velocities of order $h^{2} / \delta_{E}^{2} u_{0}$. The sum of the solutions to (2.10a) and (2.11a) are similarly valid even when the cross-shelf forcing is much greater than the alongshelf forcing.

Because of the residual effects of rotation, rotation can only be neglected for a flow in a given direction if the flow in the orthogonal direction is much less than $h^{2} \delta_{E}^{-2}$ its strength.

The solutions to (2.10) and (2.11) are, in dimensional terms,

$$
\begin{aligned}
v & =O\left(\frac{g}{6 A \rho_{0}} \frac{\partial \rho}{\partial y} h^{3}+\frac{\tau_{\text {wind }}^{y}}{A \rho_{0}} h+\frac{h^{2}}{\delta_{E}^{2}} \frac{\tau_{\text {wind }}^{x}}{A \rho_{0}} h\right) \\
& =-\frac{g}{6 A \rho_{0}} \frac{\partial \rho}{\partial y} z^{3}+\frac{1}{2 A \rho_{0}} \frac{\partial P_{0}}{\partial y} z^{2}+\frac{\tau_{\text {wind }}^{y}}{A \rho_{0}} z+\frac{1}{r}\left[-\frac{1}{\rho_{0}} \frac{\partial P_{0}}{\partial y} h-\frac{g}{2 \rho_{0}} \frac{\partial \rho}{\partial y} h^{2}+\frac{\tau_{\text {wind }}^{y}}{\rho_{0}}\right] \\
& -\left[\frac{1}{2 A \rho_{0}} \frac{\partial P_{0}}{\partial y} h^{2}+\frac{g}{6 A \rho_{0}} \frac{\partial \rho}{\partial y} h^{3}-\frac{\tau_{\text {wind }}^{y}}{A \rho_{0}} h\right] \\
& +2 \frac{h^{2}}{\delta_{E}^{2}}\left\{-\frac{\tau_{\text {wind }}^{x}}{6 A \rho_{0}} \frac{z^{3}}{h^{2}}+\frac{\tau_{\text {wind }}^{x}}{2 A \rho_{0}}\left(\frac{A}{r}+h\right) \frac{z^{2}}{h}-\frac{1}{r}\left[\frac{\tau_{\text {wind }}^{x}}{h \rho_{0}}\left(\frac{A}{r}+h\right)+\frac{\tau_{\text {wind }}^{x}}{2 \rho_{0}}\right]\right. \\
& \left.-\left[\frac{\tau_{\text {wind }}^{x}}{2 A \rho_{0}}\left(\frac{A}{r}+h\right)+\frac{h \tau_{\text {wind }}^{x}}{6 A \rho_{0}}\right]\right\}+O\left(\frac{h^{4}}{\delta_{E}^{4}} u_{0}\right)+O\left(\frac{h^{4}}{\delta_{E}^{4}} v_{0}\right),
\end{aligned}
$$

where

$$
\begin{aligned}
\frac{\partial P_{0}}{\partial y} & =-g \frac{\partial \rho}{\partial y} \frac{\left(\frac{3 h^{4}}{24}+\frac{A h^{3}}{2 r}\right)}{\left(\frac{h^{3}}{3}+\frac{A h^{2}}{r}\right)}+\tau_{\text {wind }}^{y} \frac{\left(\frac{h^{2}}{2}+\frac{A h}{r}\right)}{\left(\frac{h^{3}}{3}+\frac{A h^{2}}{r}\right)} \\
& +2 \frac{h^{2}}{\delta_{E}^{2}}\left[-\frac{\tau_{\text {wind }}^{x}}{h^{2}} \frac{\left(\frac{3 h^{4}}{24}+\frac{A h^{3}}{2 r}\right)}{\left(\frac{2 h^{3}}{3}+\frac{A h^{2}}{r}\right)}+\frac{\tau_{w i n d}^{x}}{h^{2}}\left(\frac{A}{r}+h\right)\right]+O\left(\frac{h^{4}}{\delta_{E}^{4}} \frac{\partial P_{0}}{\partial y}\right) .
\end{aligned}
$$

The solution for the alongshelf velocity is similar in structure. Because the following 
sections will be concerned primarily with the cross-shelf flow, and because the second order solution to the alongshelf velocity is algebraically formidable, only the first order solution will be given:

$$
u_{0}=\frac{\tau_{w i n d}^{x}}{A \rho_{0}} z+\frac{\tau_{w i n d}^{x}}{\rho_{0}}\left(\frac{1}{r}+\frac{h}{A}\right)+O\left(\frac{h^{2}}{\delta_{E}^{2}} v_{0}+\frac{h^{4}}{\delta_{E}^{4}} u_{0}\right) .
$$

The analytical solutions for the density, the first order solution for the cross-shelf velocity $v$, and the full solution (2.14) are compared with a numerical model with no windstress (figure 2.3 ), and with a model with a $0.1 \mathrm{~N} \mathrm{~m}^{-2}\left(\approx 10 \mathrm{~m} \mathrm{~s}^{-1}\right)$ alongshore, downwelling favorable, wind stress (figure 2.4). There is an open boundary at the seaward edge that lets the flow leave the domain undisturbed, and it is described in the appendix to this chapter. The numerical model uses the same parameterization for convection as the analytical work when the convection mixes to the bottom. The numerical model agrees nicely with (2.14), even near the offshore boundary where $h^{2} / \delta_{E}^{2} \approx 1$. The cross-shelf order one solution, $v_{0}$, which includes no rotational effects, predicts the cross-shelf velocity in the numerical model well when there is no alongshelf forcing, but both the first and second order solutions are needed when there is an alongshore windstress. When there is an alongshore windstress, the cross-shelf wind forced velocity is about one-quarter the alongshelf velocity at the seaward edge of the model.

\subsection{When Does Convection Not Mix the Entire Water Column?}

When convection extends through the water column, the flow is sluggish and the water is viscous and weakly stratified, incapable of driving a significant horizontal mass flux. Only when convection is prevented from mixing through to the bottom, and thus prevented from transmitting stress and buoyancy from the surface to the 

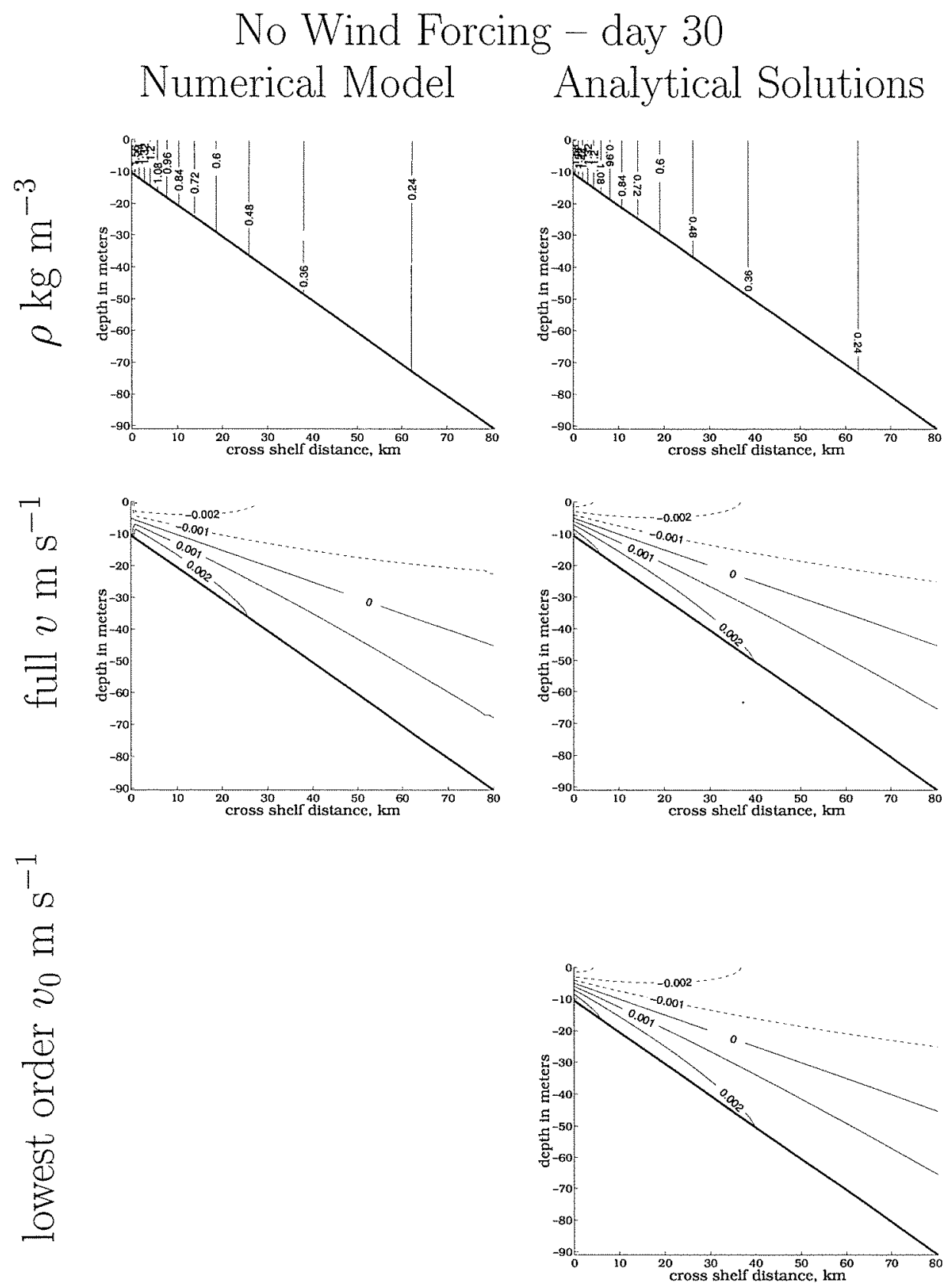

Figure 2.3: The solutions for the cross-shelf velocity and the density from $(2.14),(2.5)$ and the numerical model. The bottom panel is the lowest order analytic solution for the cross-shelf flow. Dashed contours are negative. 


\section{$0.1 \mathrm{~N} \mathrm{~m}^{-2}$ Downwelling Favorable Alongshore Wind day 30 Numerical Model Analytical Solutions}
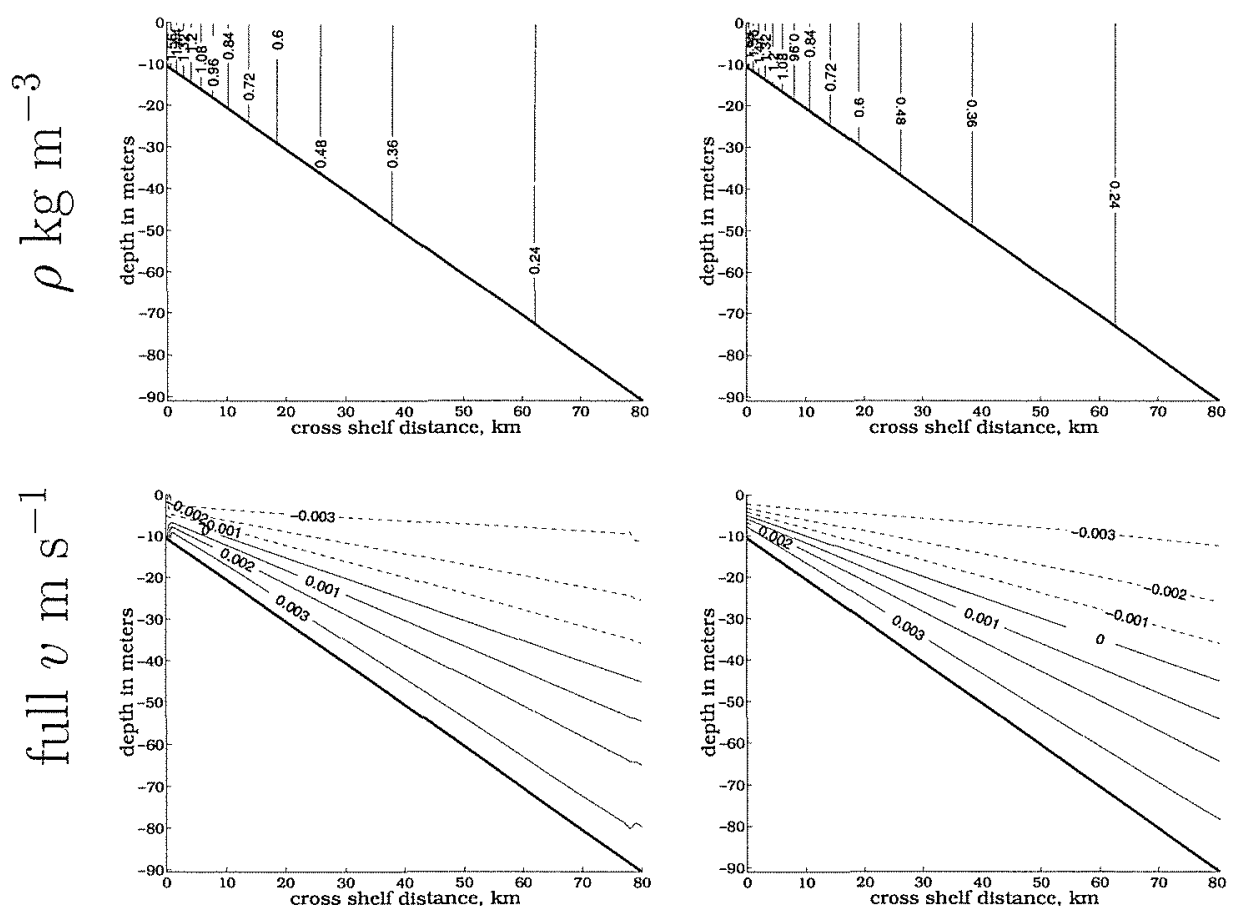

\begin{tabular}{l}
1 \\
0 \\
$\Xi$ \\
0 \\
5 \\
0 \\
0 \\
0 \\
0 \\
0 \\
0 \\
0 \\
\hline
\end{tabular}

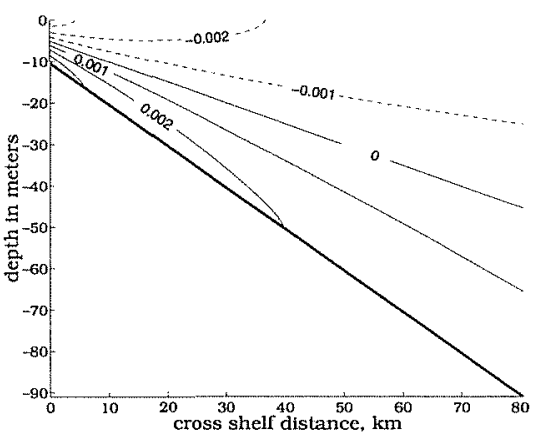

Figure 2.4: The solutions for the cross-shelf velocity and the density from (2.14), (2.5) and the numerical model. The bottom panel is the lowest order analytic solution for the cross-shelf flow. Dashed contours are negative. 
bottom, can significant stratification form and a strongly rotationally affected circulation develop. Once this occurs, significant amounts of heat can be transported offshore (c.f. chapter 3).

In the atmospheric boundary layer, turbulent convection extends from the surface to where the air becomes stably stratified, and the mean stratification of the convective region is slightly unstable [Mellor and Yamada, 1982; Stull, 1988]. This is assumed to be true in the ocean, and the mean unstable stratification formed by the cooling is compared to the stable stratification formed advectively by the circulation to determine when convection mixes to the bottom. Once the water near the bottom is stably stratified, it is assumed that convection does not penetrate through it and the diffusivity in the stably stratified water is much reduced. (In the numerical models the diffusivity of heat and momentum of the stably stratified water are calculated according to a scheme by Pacanowski and Philander [1981], though other parameterizations yield comparable results. The diffusivity of any statically unstable region is still given by 2.2.)

The unstable stratification formed by the cooling, $\rho_{z}^{\text {cooling }}$, is found from the system:

$$
\begin{array}{rl}
\rho_{t}= & \frac{\partial}{\partial z}\left(\nu \frac{\partial \rho}{\partial z}\right) \\
\nu \rho_{z}=Q & @ z=0 \\
\nu \rho_{z}=0 & @ z=-h
\end{array}
$$

In the limit of time much longer than the time of a convective overturning, $\left(h^{2} \nu^{-1}\right)$, and assuming the viscosity varies slowly compared to this time scale, the solution is

$$
\rho_{z}^{\text {cooling }}=\frac{Q}{h \nu}(z+h)
$$

The solution for $\rho_{z}^{\text {cooling }}$ is valid even if $\nu$ varies with depth. This is important, for more realistic parameterization of convection-driven mixing will prescribe mixing 
that varies with, among other things, the distance from the wall and the local vertical stratification (including that forced by advective processes).

The stratification induced by the cross-shelf circulation near the bottom, $\rho_{z}^{\text {advec }}$, can be found from:

$$
\begin{aligned}
& v \rho_{y}= \frac{\partial}{\partial z}\left(\nu \frac{\partial \rho}{\partial z}\right) \\
& \nu \rho_{z}=0 \quad @ z=-h, 0 .
\end{aligned}
$$

This system of equations assumes that $w \rho_{z}$, the vertical advective term, is negligible (which is justified below), and that the horizontal advection of density does not change the mean density of the water column, i.e. the heat/density balance is one dimensional. This is a good approximation when the depth mean density-flux divergence is much less then the density-flux divergence in most of the water column:

$$
\left|\frac{1}{h} \int_{-h}^{0} v \rho_{y} d z\right| \ll\left|v \rho_{y}\right| .
$$

Since the depth integral of $v$ is zero to enforce no flow through the coast, this can be scaled as

$$
\left|h v \rho_{y z}\right| \ll v \rho_{y}
$$

Substituting (2.18) and (2.5) into (2.21) gives

$$
\frac{t}{h^{2}} \ll \frac{1}{\nu}
$$

This is easily true for $h \approx 50 \mathrm{~m}, t \approx 10^{6} \mathrm{~s}$, and $\nu \approx 0.1 \mathrm{~m}^{2} \mathrm{~s}^{-1}$. The advectively driven stratification near the bottom can now be found by integrating (2.19a) from 
the bottom, again assuming that $\rho_{y z} \approx 0$, to get

$$
\rho_{y} \int_{-h}^{\delta-h} v d z=\left.\nu \rho_{z}^{\operatorname{advec}}\right|_{\delta-h}
$$

where $\delta$ is an arbitrary height above the bottom. To compare this result near the bottom with the stratification from cooling, the integral of $v$ must be approximated. The Taylor series approximation

$$
\int_{-h}^{\cdot \delta-h} v d z=\left.\delta v\right|_{z=-h+\delta}
$$

is accurate near the bottom if a stress condition is used as the bottom boundary condition for the momentum equation. An example stress condition is

$$
A v_{z}=r v @ \quad @ z=-h
$$

where $r$ is the bottom linear drag coefficient. Near the bottom, the length scale of velocity change is $A / r$, which for realistic values of $A\left(10^{-3}\right.$ to $\left.10^{-1} \mathrm{~m}^{2} \mathrm{~s}^{-1}\right)$ and $r\left(10^{-4} \mathrm{~m} \mathrm{~s}^{-1}\right)$ gives length scales of tens to thousands of meters, depending on whether the water column is stable or not. (This does not mean that the boundary layer extends that far, only that there is a region near the bottom where $v$ is approximately constant). If near the bottom a log-layer boundary condition is used, (2.24) has a slightly different interpretation but is till approximately valid. In the $\log$-layer, the velocity profile is

$$
v_{l l}=\frac{u^{*}}{\kappa} \ln \left(\frac{\delta}{z_{0}}\right)
$$

where $\kappa$ is von Karman's constant and $z_{0}$ is the roughness scale of the bottom [Stull, 
1988, p. 376]. Equation (2.24) is then tantamount to approximating

$$
\left.\int_{-h}^{\delta-h} v_{l l} d z \approx \delta v_{l l}\right|_{z=\delta-h}
$$

Integrating (2.26) directly and taking the ratio of the integral and (2.27) allows one to estimate the error caused by neglecting the log layer:

$$
\left.\delta v_{l l}\right|_{z=\delta-h}\left(\int_{-h}^{\delta-h} v_{l l} d z\right)^{-1}=\ln \left(\frac{\delta}{z_{0}}\right)\left(\ln \left(\frac{\delta}{e z_{0}}\right)\right)^{-1}
$$

Assuming $\delta \approx 1 \mathrm{~m}$ and $z_{0} \approx 10^{-2} \mathrm{~cm}$, this ratio is about 1.16 , indicating that $(2.24)$ over-estimates the transport by about $16 \%$. The stratification formed by advection near the bottom, (2.23), can now be written with (2.24) as

$$
\left.\rho_{z}^{\operatorname{advec}}\right|_{\delta-h}=\frac{\left.\delta \rho_{y} v\right|_{z=\delta-h}}{\nu}
$$

Again, this solution is valid even if $\nu$ varies with depth.

At this point the assumption that $w \rho_{z}$ is negligible compared to $v \rho_{y}$ can be checked. $w$ is scaled as

$$
w=O\left(v \frac{\partial h}{\partial y}\right)
$$

so $w \rho_{z}$ is much less than $v \rho_{y}$ when

$$
\rho_{y} \gg \frac{\partial h}{\partial y} \rho_{z}
$$

Substituting (2.29) for $\rho_{z}$ and rearranging the resulting equation leads to

$$
1 \gg \frac{(z+h) v}{\nu} \frac{\partial h}{\partial y}
$$

which is easily true for the cases presented here where $v$ is $O\left(10^{-2} \mathrm{~m} \mathrm{~s}^{-1}\right), \partial h / \partial y$ is 
$\mathrm{O}\left(10^{-3}\right)$ and $\nu$ is $\mathrm{O}\left(10^{-1}\right.$ to $\left.10^{-3} \mathrm{~m} \mathrm{~s}^{-2}\right)$.

The total stratification forced by the cooling and advection is, near the bottom and from (2.18) and (2.29),

$$
\left.\frac{\partial \rho}{\partial z}\right|_{\delta-h}=\frac{\delta}{\nu}\left(\left.\frac{\partial \rho}{\partial y} v\right|_{\delta-h}+\frac{Q}{h}\right) .
$$

The water column is thus stably stratified near the bottom when

$$
-\left.\frac{\partial \rho}{\partial y} v\right|_{\delta-h} \geq \frac{Q}{h}
$$

which is when the divergence of the advective flux of density is greater than the divergence of the density flux caused by cooling. This can be solved for the minimum velocity needed to make the water stably stratified near the bottom, $v_{c}$,

$$
v_{c}=-\frac{Q}{h}\left(\frac{\partial \rho}{\partial y}\right)^{-1} .
$$

This can then be written

$$
v_{c}=\frac{h}{t}\left(\frac{\partial h}{\partial y}\right)^{-1}
$$

with $\partial \rho / \partial y$ from (2.6). Once the cross-shelf velocity near the bottom exceeds $v_{c}$, convection cannot reach the bottom, so the enhanced mixing due to convection cannot reach the bottom. The velocity $v_{c}$ should be compared with the velocity either at the bottom, when $A$ is constant, or at the top of the log-layer, when there is one. The diffusivity of density, $\nu$, does not enter into (2.35) or (2.36) because both the unstable stratification forced by the cooling, (2.18), and the stable stratification forced by the circulation, (2.29), scale linearly with $\nu^{-1}$. It takes a smaller cross-shelf velocity to make the water stably stratified as time increases because as time increases the cross-shelf density gradient increases, increasing the stabilizing stratification caused by a given cross-shelf velocity (2.29). 
To test the criterion (2.36), five numerical model runs were made, each with the same geometry and surface cooling as previously described, and each with initially homogeneous water. The model uses the exact same vertical viscosity and density diffusion as the analytic work. In one model run, no wind forcing was used, and in the other four an $0.1 \mathrm{~N} \mathrm{~m}^{-2}\left(\approx 10 \mathrm{~m} \mathrm{~s}^{-1}\right)$ wind was applied in the onshore, offshore, or one of the alongshore directions. The cross-shelf velocities near the coast in the model within 0.3 days of the onset of stable stratification are plotted in figure 2.5 after being scaled by (2.36). All five models first become stratified near the coastal boundary, and in all five cases the scaled cross-shelf velocity is very nearly one where the water becomes stably stratified. Thus (2.35) and (2.36) are good measures of when the cross-shelf current can prevent convection from reaching the bottom.

In section 2.3, an analytic solution was found which predicted the flow fields of the numerical model well, and in this section a criterion was found for the cross-shelf velocity needed to make the water column stable. It is possible to combine equations (2.14) and (2.36) to provide an expression for the onset of stable stratification in the model, but the result is algebraically formidable. However, in the limit of $A r^{-1} \gg h$, the near bottom velocity can be written as:

$$
v_{\text {bot }}=\frac{h^{3}}{24 A}\left(-\frac{g}{\rho_{0}} \frac{\partial \rho}{\partial y}+\frac{f \tau_{\text {wind }}^{x}}{A \rho_{0}}\right)-\frac{h \tau_{\text {wind }}^{y}}{6 A \rho_{0}}
$$

This scale for the bottom velocity is compared with the velocity from the numerical model, the full analytic solution (2.14), and the criterion for the onset of stability (2.36) in figure 2.6. The velocity scale $v_{b o t}$ compares well with both the numerical model and (2.14), and when these three estimates of the near bottom velocity exceed (2.36) the increased cross-shelf currents associated with the onset of stable 


\section{Scaled Cross-Shelf Velocities Shaded where $v / v_{c}>1$}
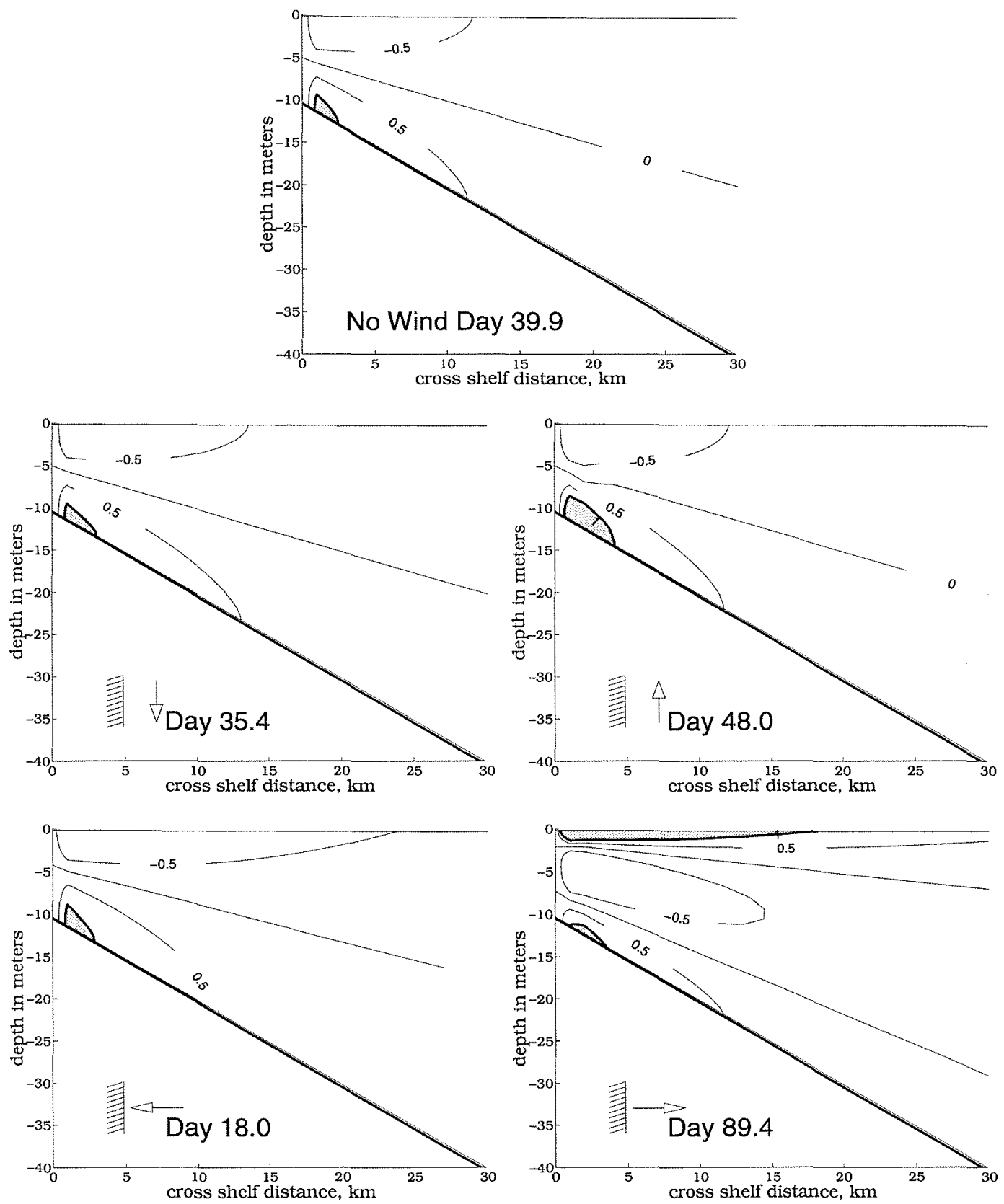

Figure 2.5: Scaled cross-shelf velocity just before convection is prevented from mixing to the bottom for five model runs with different wind forcing. The arrow indicate the direction of the wind relative to the coast. Only the inner $30 \mathrm{~km}$ of the model domain are shown. 


\section{Cross-Shelf Bottom Velocity Where Water First Becomes Stable, $3 \mathrm{~km}$ offshore}
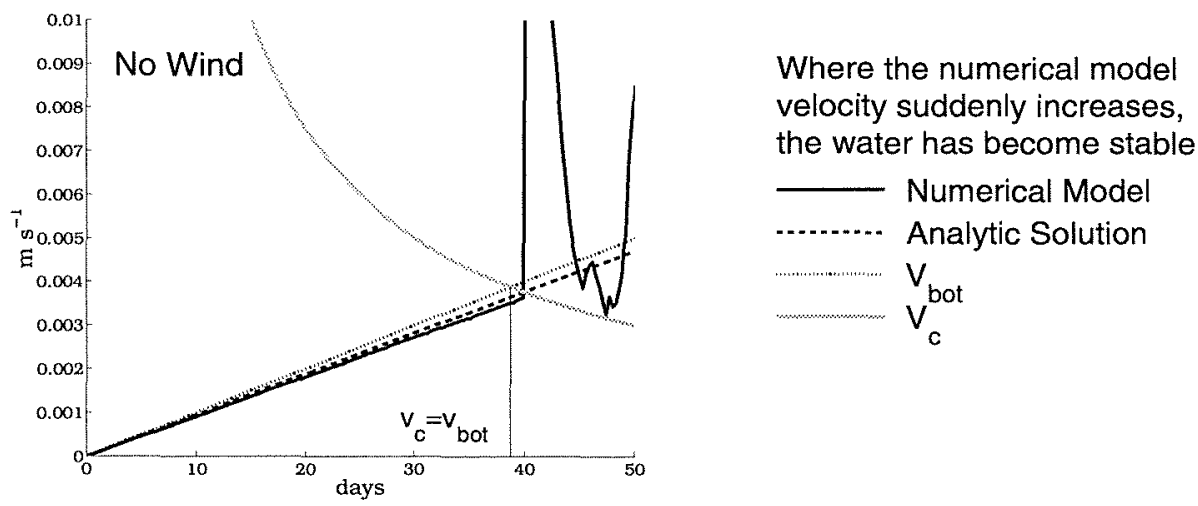

the water has become stable

- Numerical Mode

Analytic Solution
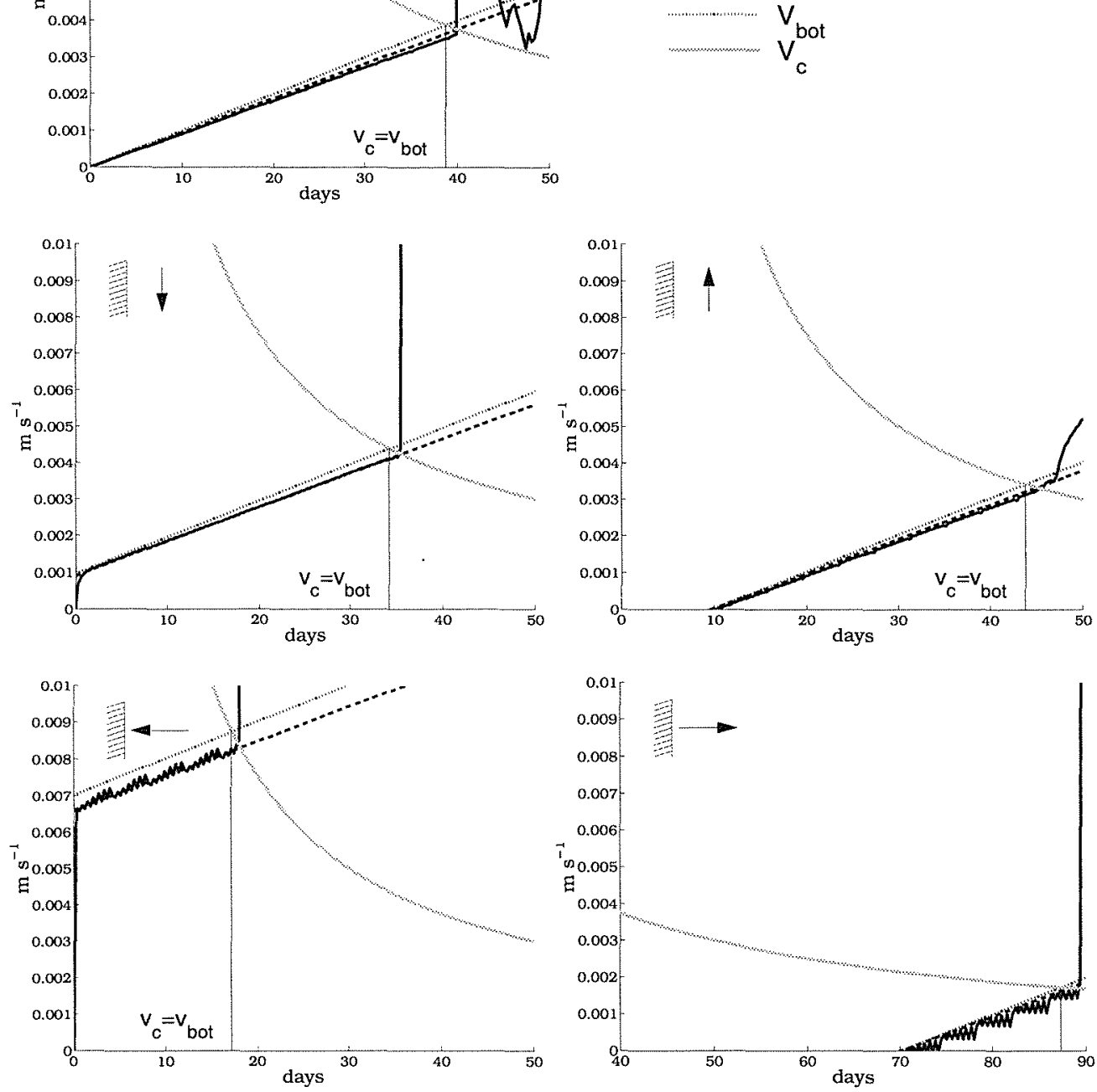

Figure 2.6: The cross-shelf bottom velocity for the five numerical model runs shown in figure 2.5. the velocity is computed $3 \mathrm{~km}$ offshore, where the model first becomes stable. The thin vertical line marks where $v_{c}=v_{b o t}$, the criterion for stability given in (2.38). The sharp increase in the cross-shelf velocity in the numerical model indicates the onset of stable stratification near the bottom. The arrows represent the wind direction relative to the coast. 
stratification are seen. ${ }^{1}$ The velocity scale $v_{b o t}$ exceeds $v_{c}$ when

$$
1=\frac{g Q}{24 A \rho_{0}}\left(\frac{\partial h}{\partial y}\right)^{2} t^{2}+\frac{h^{2} f \tau_{\text {wind }}^{x}}{24 A^{2} \rho_{0}} \frac{\partial h}{\partial y} t-\frac{\tau_{\text {wind }}^{y}}{6 A \rho_{0}} \frac{\partial h}{\partial y} t .
$$

The time for the onset of stability can be found by solving (2.38) with the quadratic equation. These times are marked in figure 2.6. From (2.38) it can be seen that the water will become stable where $A$ is the least. When the mixing is driven by the cooling alone and increases as the four thirds power of depth, (2.2), this occurs where the water is shallowest (in the numerical model stability first occurs 3 gridpoints (or $3 \mathrm{~km}$ ) from the coastal wall because of the boundary layer associated with the coastal wall). However, in a real ocean the mixing may increase as the water gets shallower (if, for instance, the mixing is driven by surface gravity waves), so this result may well break down as the water becomes shallow. Furthermore, (2.38) depends on a cross-shelf gradient of density which increases as $h^{-2},(2.6)$. As the water become shallower and the cross-shelf density gradient increases, it is likely that some form of lateral mixing would reduce the gradient, reducing the effectiveness of advective restratification and moving the location of the first stable stratification offshore. Equation (2.38) also illustrates that an alongshelf wind is less able to make the water stable than a comparable cross-shelf windstress by a factor $O\left(h^{2} / \delta_{E}^{2}\right)$, because the alongshelf wind generates a cross-shelf current that is $O\left(h^{2} / \delta_{E}^{2}\right)$ weaker than an equivalent cross-shelf windstress. The importance of a density-driven circulation relative to wind-driven circulation is seen to increase with time, because the strength of the density driven circulation increases in time while the wind driven circulation remains steady.

\footnotetext{
${ }^{1}$ The velocity in the numerical model exhibits small $\Delta t$ noise, which is damped every 3.6 days by an Euler timestep. Removing it more often would introduce spurious numerical diffusion.
} 
In the absence of wind, the water is predicted to become stable by (2.38) when

$$
\begin{aligned}
t & =\sqrt{\frac{24 A \rho_{0}}{g Q}}\left(\frac{\partial h}{\partial y}\right)^{-1} \\
& =38.4 \text { days for the no wind case. }
\end{aligned}
$$

The numerical model becomes stable at about 39.9 days, and the discrepancy arises not from the failure of the scale for $v_{c}$ but, as can be seen from figure 2.6, from the approximation of the bottom velocity by (2.37). An $0.1 \mathrm{~N} \mathrm{~m}^{-2}$ alongshore windstress drives cross-shelf velocities of about $10^{-3} \mathrm{~m} \mathrm{~s}^{-1}$. This retards the onset of stable stratification by 8.1 days when the alongshore wind is upwelling favorable, and advances the onset by 4.5 days when the wind is downwelling favorable. The change in time from the no wind case is unequal because, as can be seen from (2.38) and figure $2.6, v_{c}$ scales as $t^{-1}$, so reducing the bottom velocity by a constant changes the time when $v_{c}=v_{b o t}$ by more than increasing the bottom velocity. An $0.1 \mathrm{~N} \mathrm{~m}^{-2}$ cross-shelf wind drives cross-shelf currents almost seven times greater then the equivalent alongshelf windstress, and so changes the time of the onset of stable stratification by more than the equally strong alongshelf wind. An offshore wind retards the onset of stable stratification by 49.5 days while an onshore wind advances it by 21.9 days.

The solution for the near-bottom velocity needed to make the water stably stratified does not depend on the magnitude or shape of the eddy viscosity $A$ or density diffusion $\nu$ except to the extent that a vanishing eddy viscosity near the bottom introduces errors into (2.24), and these errors are shown to be small in (2.28). The estimates of the actual cross-shelf velocity in (2.37) and (2.14) do, however, depend on the magnitude and the vertical profile of $A$. From the expression for $V_{b o t},(2.37)$, the effect of changing the magnitude of $A$ can be estimated: the cross-shelf flow driven by cross-shelf winds and density gradients scales as $A^{-1}$, and the cross-shelf flow driven by alongshelf winds scales as $A^{-2}$. 
The cross-shelf velocity profile also depends on the vertical structure of $A$. Observations of the turbulent velocity for free convection find that it varies with height above the boundary and goes towards zero at the boundaries [Mellor and Yamada, 1982; Stull, 1988]. Realistic mixing schemes account for this by varying $A$ with the distance from the boundary. The level 2 turbulence closure presented in Mellor and Yamada [1982] varies the mixing approximately quadratically, so that

$$
A \propto-\frac{w^{*}}{h} z(z+h)
$$

[Nunes Vas and Simpson, 1994]. Another approach which has been used is to assume that the vertical mixing varies linearly with distance away from the wall,

$$
A \propto w^{*}\left(\frac{h}{2}-\left|z+\frac{h}{2}\right|\right)
$$

which could be regarded as an extension of the log-layer throughout the water column. These and other schemes are presented and discussed in the context of wallgenerated turbulence by Lentz [1995] ((2.40) reduces to the cubic case in Lentz when $w^{*}$ is used to scale the turbulent velocity). To get a rough idea of the sensitivity of the near bottom velocity to the vertical profile of $A$, the cross-shelf velocity is found for the two profiles given in (2.40) and (2.41), and for a constant eddy viscosity. Each will be normalized so the depth average $A$ will be $0.25 w^{*} h$, as in (2.2). Since the viscosities in (2.40) and (2.41) go to zero at the bottom and top, the full numerical model cannot be used to solve for the velocity profiles. Instead, (2.10) is solved by a Runge-Kutta solver with the boundary conditions

$$
\begin{aligned}
\frac{\partial v}{\partial z} & =0 @ z=-z_{0}, \\
v & =0 @ z=-h+z_{0},
\end{aligned}
$$

where $z_{0}$ is a surface roughness scale, to which the solutions are not very sensitive 
[Lentz, 1995]. Herein, $z_{0}=10^{-2} \mathrm{~m}$. The constant eddy viscosity solution is that of (2.14), and as long as $A r^{-1} \gg h$, it is not sensitive to the value of $r$. Figure 2.7 shows $A$ and the cross-shelf velocity for the three vertical eddy viscosity profiles. The cross-shelf velocities in 13 meters of water are found for day 39.6 of the no wind case, which is where and just before the water column becomes stable in the vertically uniform $A$ case. The only qualitative difference in the cross-shelf velocity profiles is at the bottom, where the velocities in the depth variable $A$ cases go to zero in a log-layer. This is caused by the boundary condition (2.42), and as discussed above $v_{c}$ is then compared to the velocity many $z_{0}$ above the bottom. The velocity one meter above the bottom, which is approximately where the peak cross-shelf velocities occur in the depth dependent $A$ cases, is about $30 \%$ lower than the near bottom velocities in the constant $A$ case. The velocity is about $12 \%$ lower when $A$ is given by (2.40) then when given by (2.41). (In both cases (2.28) accurately predicts the error in (2.24), about 18\%.) Since the changes in the near bottom velocities for the more realistic mixing profiles are relatively small, it seems reasonable to use (2.37) $(2.2)$, and (2.35) to estimate the time for the onset of stable stratification in the ocean. In a case with no wind, a $30 \%$ reduction in the near bottom cross-shelf velocity implies a $20 \%$ increase in the time until $v_{b o t}>v_{c}$ and the water column becomes stable (2.39).

\subsection{When Convection no Longer Mixes to the Bottom}

When convection extends to the bottom, pressure gradients and wind forcing can be balanced directly against bottom friction. Once the velocity near the bottom exceeds $v_{c}$ and convection no longer mixes to the bottom, the viscosity near the bottom is reduced to levels appropriate for neutral and stably stratified waters. This allows rotation to affect the circulation strongly. Wind will then force a transport to the 

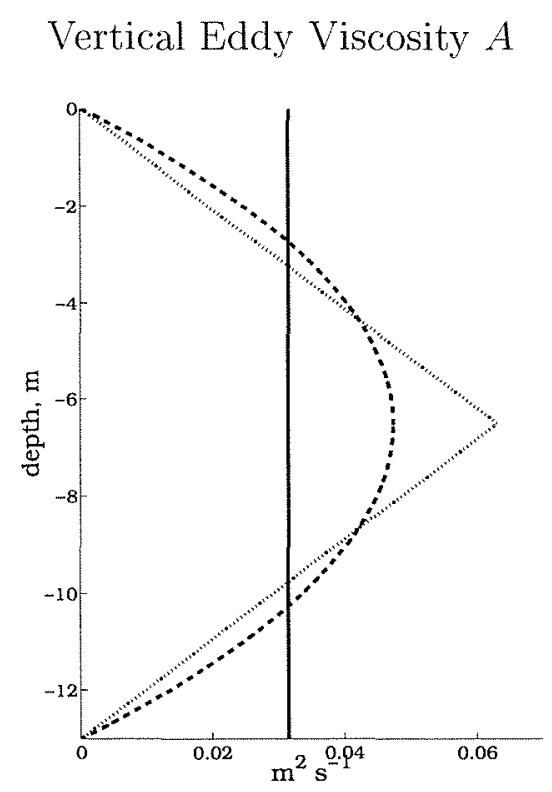

Cross-Shelf Velocity $v$

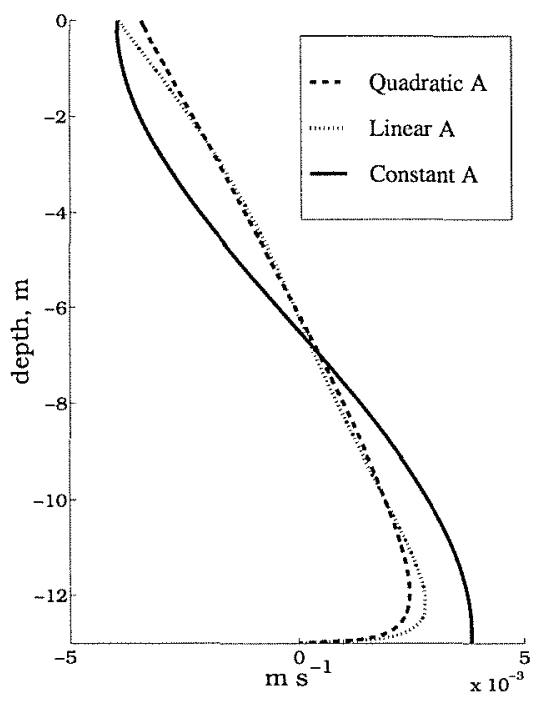

Figure 2.7: The vertical eddy viscosity and the cross-shelf velocity profile for three different profiles of vertical eddy viscosity. Day 39.6 of the no wind case.

right of the wind (when $f>0$ ), and cross-shelf density gradients will drive velocities by thermal wind. If this new flow field maintains a bottom velocity greater than $v_{c}$, convection will not remix to the bottom and the rotationally affected circulation will persist.

When the model with no wind forcing makes the transition to stable stratification near the bottom, the offshore flow in the bottom boundary layer remains strong enough to prevent convection from mixing back down to the bottom, and an alongshore flow in thermal wind balance develops (figure 2.8). The cross-shelf flow after the onset of stability is also shown scaled by $v_{c}$ in figure 2.8 , and the scaled velocity is greater than one everywhere the water column is stable. In a model which allows alongshore variability in the flow, the alongshore flow then quickly forms baroclinic instabilities which develop into geostrophic turbulence which extends over the entire model domain (chapter 3). This unsteady flow field can transport cold dense water offshore quickly enough to balance the surface cooling, and this balance is examined 


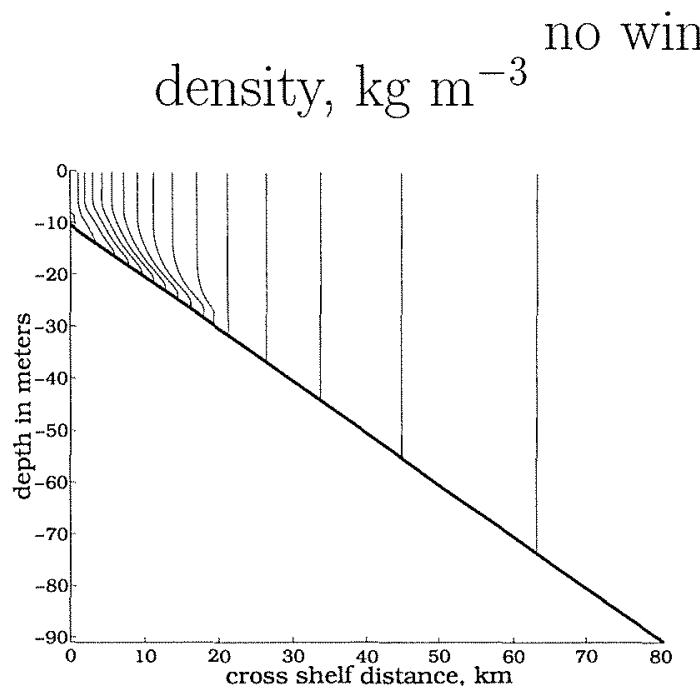

case

alongshelf velocity $\mathrm{m} \mathrm{s}^{-1}$
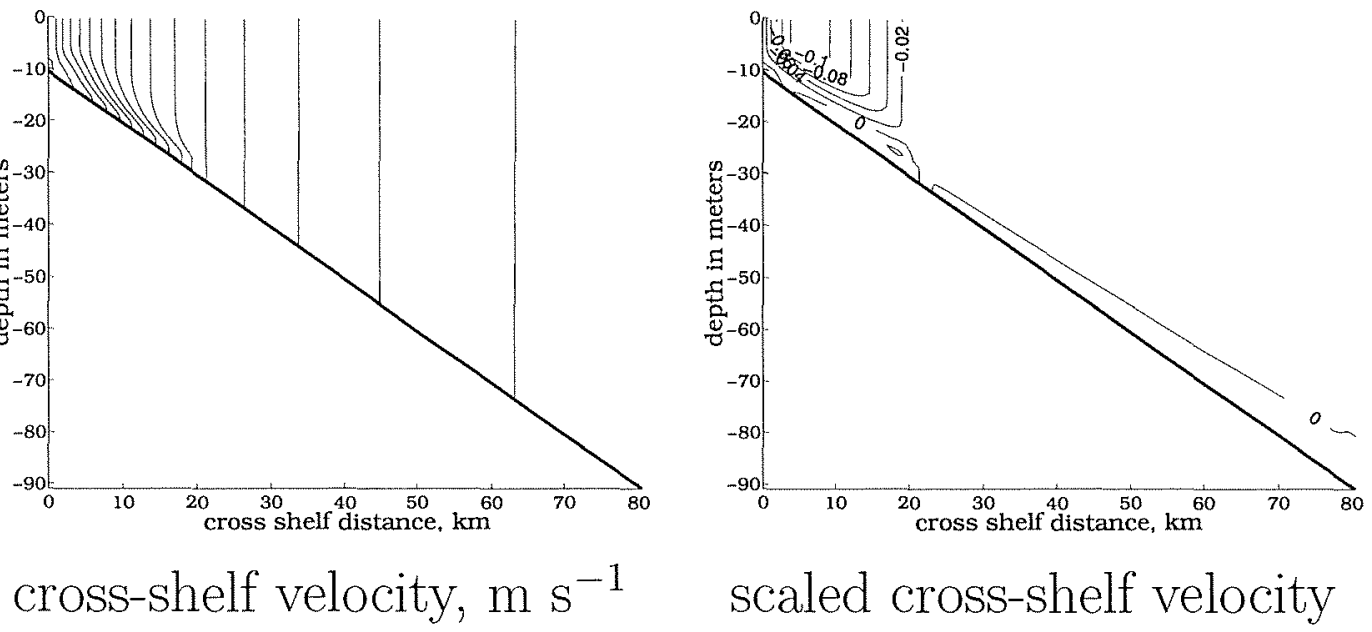

scaled cross-shelf velocity
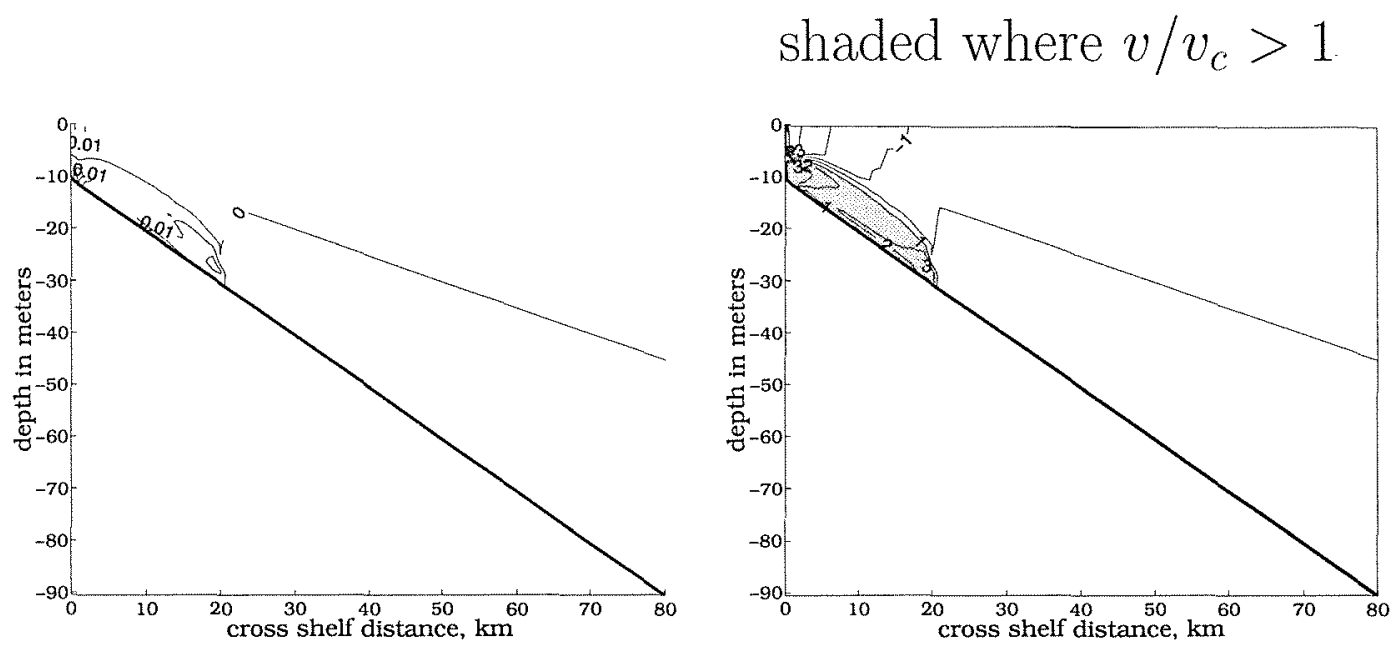

Figure 2.8: The density, the alongshore velocity, and the dimensional and scaled crossshelf velocity for the numerical model with no wind forcing at day 46 , shortly after the onset of stable stratification. The contour interval of the density is $0.12 \mathrm{~kg} \mathrm{~m}^{-3}$. 


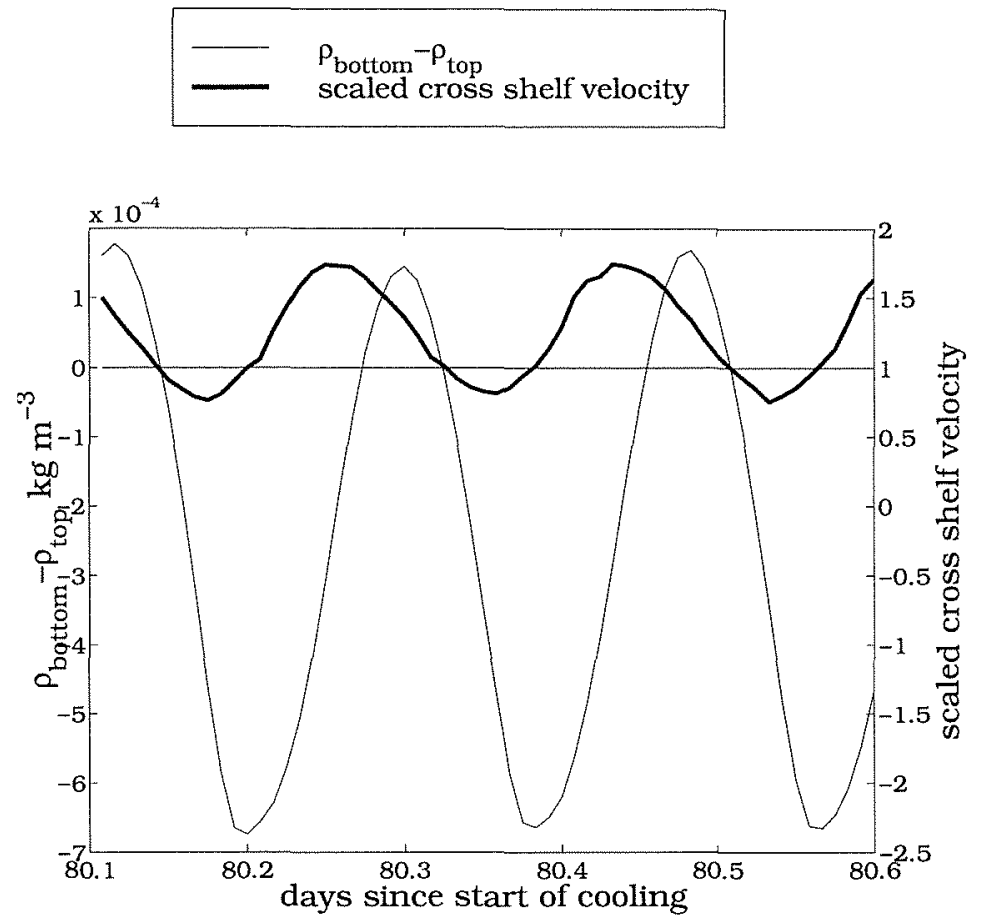

Figure 2.9: $\rho_{\text {bottom }}-\rho_{\text {top }}$ and scaled near bottom cross-shelf velocity $17 \mathrm{~km}$ offshore of the coast. The model is run with a $0.1 \mathrm{~N} \mathrm{~m}^{-2}$ alongshore upwelling favorable wind.

in chapter 3.

In contrast, the model with the alongshore upwelling favorable wind does not retain its stable stratification once it gains it. When the downwelling circulation driven by the cross-shelf density gradient exceeds $v_{c}$ near the bottom, convection is prevented from reaching the bottom and, as before, rotation begins to affect the flow. Unlike the no wind case, the rotationally affected flow in this case works to reverse the velocity near the bottom in waters deeper than 15 meters, for the wind driven flow in the bottom boundary layer with an upwelling favorable wind is towards the coast. Once the upwelling flow begins to develop, the near bottom velocity falls below $v_{c}$ and convection again reaches the bottom, allowing the density gradient driven flow to resume and prevent convection from reaching the bottom again. This 
oscillation is illustrated in figure 2.9 , in which the water column can be seen to become unstably stratified as the scaled cross-shelf velocity falls below one.

\subsection{When Cooling Falters}

When the cooling falters and there is no longer convection to mix to the bottom, rotation will again affect the circulation. When the cooling resumes, convection will not mix to the bottom if the cross-shelf velocity near the bottom exceeds $v_{c}$. To illustrate this, a numerical model is run with cooling and a $0.1 \mathrm{~N} \mathrm{~m}^{-2}$ downwelling favorable alongshore wind, and the cooling is interrupted for 2 days after 15 days of cooling. In figure 2.10 the scaled cross-shelf velocity of this model is plotted before, during, and after the pause in cooling, along with plots from the same time in the model with no pause in cooling but the same wind forcing. In the model with the pause in cooling, a wind driven downwelling circulation develops when the cooling ceases, and this circulation drives an offshore velocity near the bottom which is greater than $v_{c}$. Thus, when the cooling resumes, convection does not mix to the bottom, and the alongshore wind continues to drive a downwelling circulation. The model without the pause in cooling remains mixed through to the bottom by convection in all three plots, and this does not change until 35.4 days after the onset of cooling when the density driven cross-shelf circulation becomes strong enough to prevent convection from reaching the bottom (c.f. section 2.4).

\subsection{Conclusions}

If the cooling over a continental shelf is strong enough that

$$
h<\pi^{\frac{3}{2}}\left(\frac{g Q}{\rho_{0} f^{3}}\right)^{\frac{1}{2}}
$$




\section{Scaled Cross-Shelf Velocity}

Shaded Where $v / v_{c}>1$

\section{With Pause in Cooling No Pause in Cooling}
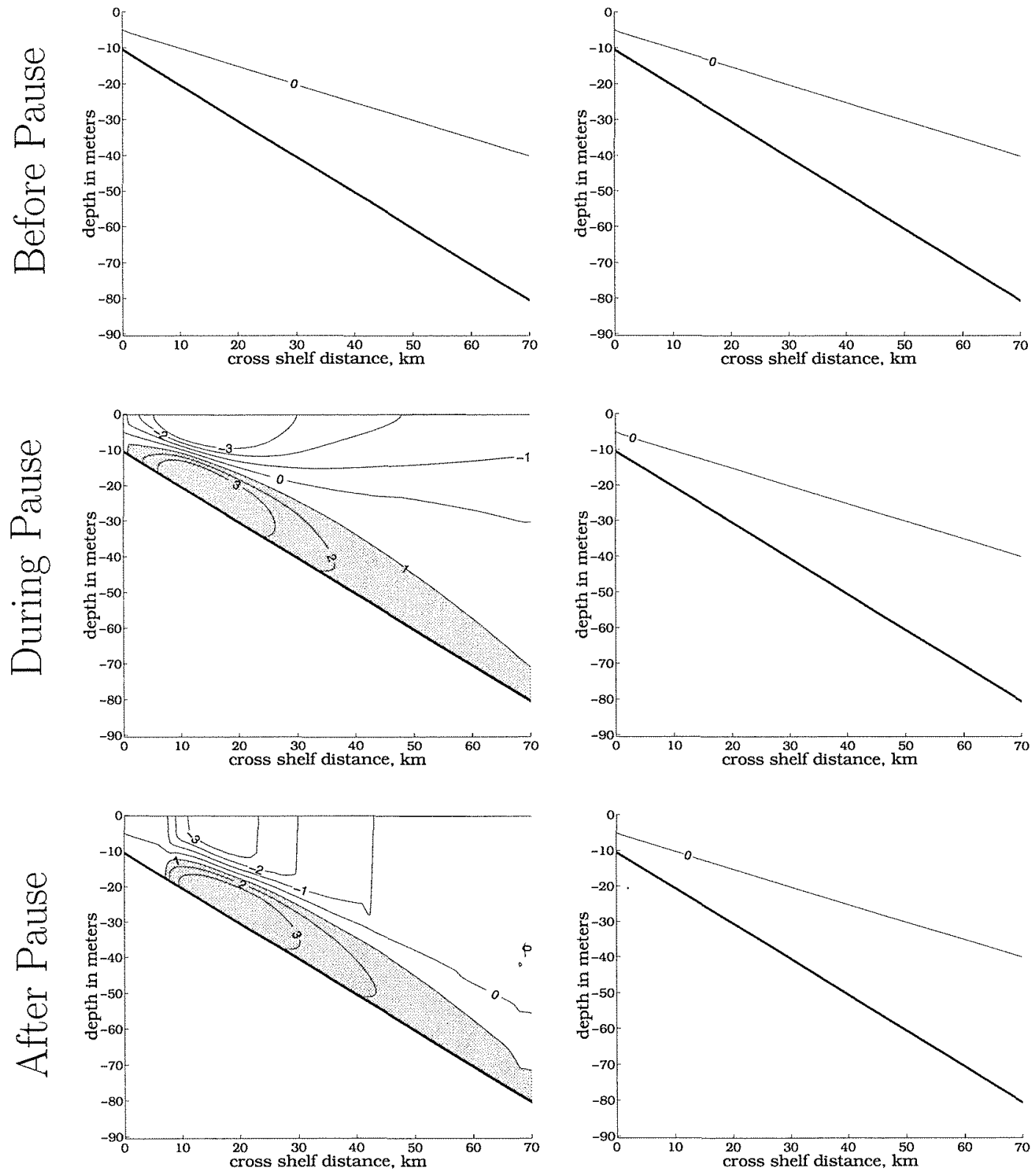

Figure 2.10: Scaled cross-shelf velocity for models with alongshore downwelling favorable winds. The left hand plots are for a model with a pause in the cooling, the right hand plots are for a model without a pause in the cooling. 
convection can mix the water column in less than an inertial period, and if the cross-shelf velocity near the bottom is less than

$$
v_{c}=-\frac{Q}{h}\left(\frac{\partial \rho}{\partial y}\right)^{-1}
$$

it will. If the water column is mixed to the bottom by convection in less than an inertial period, pressure gradients and wind forcing can be balanced by bottom friction. If these forces are balanced by bottom friction, the familiar rotationally dominated flow, with boundary layer transport to the right of the wind $(f>0)$, interior flow along isobars, and viscous effects confined to boundary layers, will not occur. Instead, viscous effects will dominate the entire water column, with flow nearly down-wind and down pressure gradient. If the cross-shelf velocity near the bottom is greater than $v_{c}$ the water column stabilizes and cooling-driven mixing will not reach the bottom and rotation can affect the flow.

These simple rules can lead to complex behaviors because the cross-shelf velocity near the bottom depends on whether convection mixes to the bottom or not. Thus, as seen in section 2.6, convection can be prevented from mixing to the bottom by a cross-shelf flow that exists because convective mixing does not reach the bottom. Conversely, in 2.5, a model run is presented in which the convective mixing is prevented from reaching the bottom by the cross-shelf flow, allowing a new cross-shelf flow to be established which then allows mixing to reach the bottom again. This is repeated ad infinitum. The former case illustrates that the dynamics of the flow can depend on the past history of the forcing, while the later example shows that the dynamics can oscillate between two different regimes.

These complex behaviors are a symptom of the non-linearities of a continental shelf undergoing surface cooling. The chief non-linearity addressed here is the dramatic change in vertical mixing that can be brought about by a small change in the vertical stratification, from stable to unstable. The transition from unstable to stable stratification occurs when the near bottom flow exceeds $v_{c}$, and thus even 
small changes in the wind or thermal forcing can cause dramatic changes in the flow field if they nudge the near bottom flow past $v_{c}$. 


\subsection{Appendix}

The numerical model is SPEM 5.1, an enhanced version of the model described in Hedstrom [1994]. This version of SPEM uses finite differences in the vertical and uses an implicit mixing scheme. The model is now built on a full 3D Arakawa C grid and is a rigid lid primitive equation model.

The model uses a modified sigma coordinate system in the vertical, in which the vertical resolution near the top and bottom is kept constant while the interior vertical resolution scales with the water depth [Song and Haidvogel, 1994]. The model was run with thirty levels in the vertical, concentrating eight levels in both the top and bottom ten meters in order to resolve the boundary layers.

I made two changes to the numerics of the model which have improved the computational speed by about an order of magnitude for these runs. The $2 \mathrm{D}$ case was changed so that the vorticity is no longer inverted by a general elliptic equation solver, but by a tri-diagonal solver. This improves the speed of the model by a factor of 3 . This has no effect on the model solution. The implicit vertical mixing scheme can now run at a shorter timestep than the rest of the model. This is necessary because of the extremely high diffusivities needed to model convection. This approach works if the time scale of diffusion, $\Delta z^{2} \nu^{-1}$, is much less than the timescale over which the flow and density fields evolve, which is true for these runs. The general approach used is to write the equations of the model (using the scalar equation as an example) with all but the diffusion terms as forcing terms, e.g.

$$
\rho_{t}-\partial_{z}\left(\nu \partial_{z}(\rho)\right)=\nabla(\vec{u} \rho)+\mathcal{F}_{\rho}
$$

where $\mathcal{F}_{\rho}$ are the physical forcing terms. The left hand side is then computed on a smaller time step assuming the right hand side is a constant, and the right hand side is re-evaluated at the coarser time step. This keeps the solution to the equation well conditioned, keeping numerical noise from corrupting the model. The same implicit 


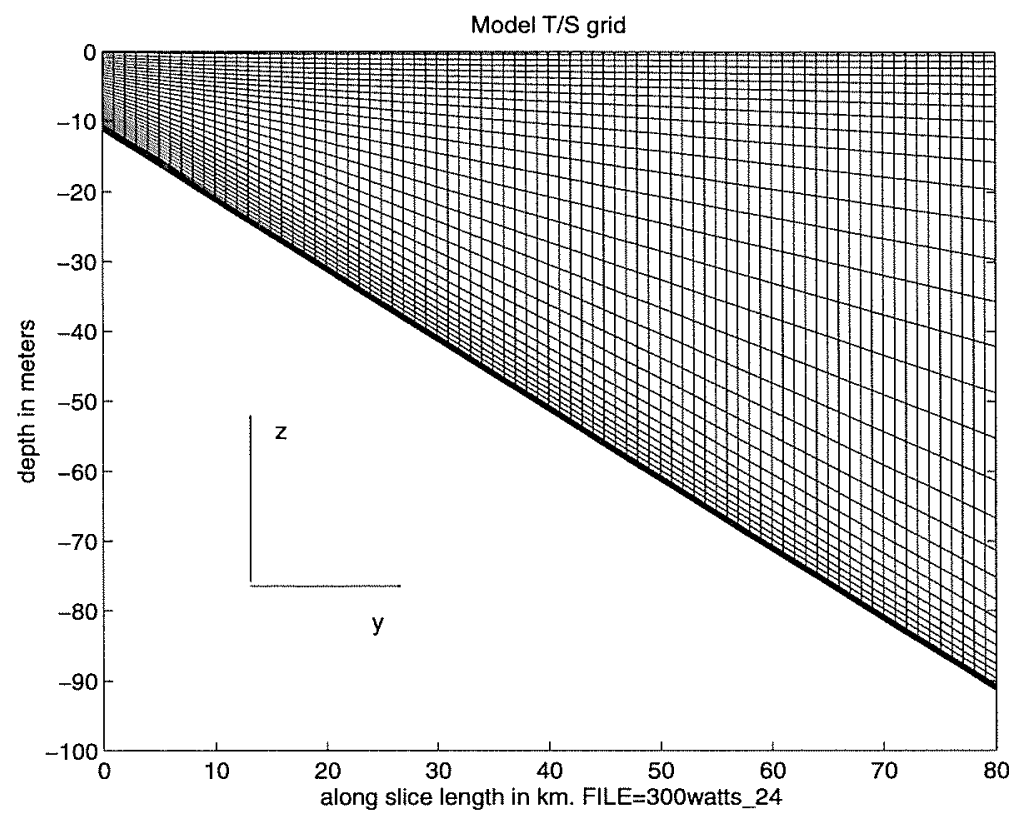

Figure 2.11: The model geometry that is used. The intersection of the lines are the density points, and the rest of the points are staggered as in an Arakawa $\mathrm{C}$ grid.

Crank-Nicolson scheme is used to evaluate the diffusion equation as originally used in SPEM 5.1. The slow time step is 80 seconds and the fast one $262 / 3$ seconds, giving another factor of 3 improvement in speed for the parameter range used below.

The model domain is two dimensional, $80 \mathrm{~km}$ in the cross-shelf direction with $1 \mathrm{~km}$ resolution offshore and a slope of one part in a thousand, so that the water depth ranges from 10 meters to 90 meters. Figure 2.11 displays the grid of the model.

The seaward boundary is open. The boundary conditions assume that the dynamics are quasi-balanced, i.e. that the flow field can be diagnosed from the mass field. This is, of course, an approximation, for if it were true, there would be no internal gravity waves. An open boundary condition at the seaward edge consistent with this is no cross-shelf gradients in $u$ and $v$ and no cross-shelf gradients in the gradients of density, so $\partial^{2} \rho / \partial y^{2}=0$. This insures that where there is no cross-shelf 
variation in $u$ and $v$, there is no cross-shelf variation in the pressure gradients which force the flow. The grid point immediately outside the model domain is assumed to have the same depth as the grid point immediately inside the domain. Several runs were done to compare the open boundary to a case where the domain was extended, and the comparison was good (errors in the near boundary velocity $<5 \%$ ). The lack of any flow irregularities at the boundary also supports the validity of this scheme.

The model was run with the Pacanowski and Philander (1981) Richardson number dependent mixing scheme. This mixing scheme has given good results in previous studies [Allen and Newberger, 1996; Nunes Vas and Simpson, 1994]. The diffusivity and viscosity at each stably stratified point are given as a function of the gradient Richardson number:

$$
\begin{aligned}
A & =\frac{10^{-2}}{(1+5 R i)^{2}}+10^{-4} \mathrm{~m} \mathrm{~s}^{-2} \\
\nu & =\frac{A}{(1+5 R i)}+10^{-5} \mathrm{~m} \mathrm{~s}^{-2}
\end{aligned}
$$

where

$$
R i=\frac{g \rho_{z}}{\rho_{0}\left(u_{z}^{2}+v_{z}^{2}\right)}
$$

Convective adjustment is handled at each unstably stratified point by an enhanced eddy diffusivity, with a scheme described in section 2.2 .

The heat flux at the surface has been converted to a mass flux for the model runs described here. If one assumes a linear equation of state, this is straightforward. Multiplying the boundary condition on temperature,

$$
\nu T_{z}=\frac{F}{C_{p} \rho_{0}} \quad @ z=0
$$

by the thermal expansion coefficient, $\alpha=\frac{\partial \rho}{\partial T}$, gives a boundary condition on density 


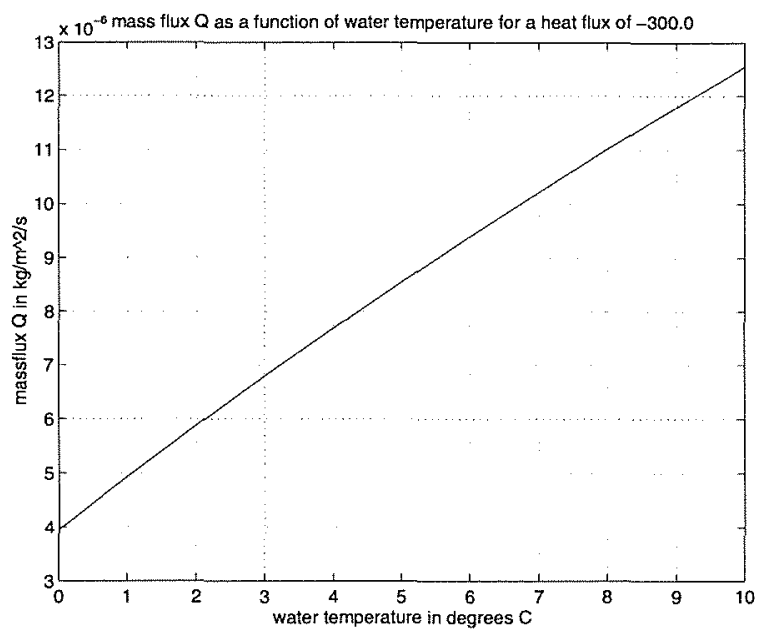

Figure 2.12: This is a plot of the equivalent mass flux for a heat flux of 300 Watts per meter squared as a function of water temperature.

of

$$
\nu \rho_{z}=Q \quad @ z=0
$$

where $F$ is the heat flux in watts per meter squared, and $Q$ the equivalent surface density flux in kilograms per meter squared per second. A problem arises from the fact that $\alpha$ is a strong function of temperature, unlike $\rho_{0}$ and $C_{p}$. For a given heat flux, the equivalent mass flux is 2.2 times greater at $5^{\circ} \mathrm{C}$ than at $0^{\circ} \mathrm{C}$. Figure 2.12 is a plot of the equivalent mass flux for a heat loss of $300 \mathrm{~W} \mathrm{~m}^{-2}$ as a function of water temperature. I use a mass flux of $7 \times 10^{-6} \mathrm{~kg} \mathrm{~m}^{-2} \mathrm{~s}^{-1}$, which is equivalent to $300 \mathrm{~W} \mathrm{~m}^{-2}$ into $3{ }^{\circ} \mathrm{C}$ water or $170 \mathrm{~W} \mathrm{~m}^{-2}$ into $10^{\circ} \mathrm{C}$ water. 


\subsection{Bibliography}

Allen, J. S. and P. A. Newberger, Downwelling Circulation on the Oregon Continental Shelf. Part I: Response to Idealized Forcing, J. Phys. Oceanogr., 26, 2011-2035, 1996.

Brown, W. S. and R. C. Beardsley, Winter Circulation in the Western Gulf of Maine: Part I cooling and Water Mass Formation, J. Phys. Oceanogr., 8(2), 265-277, 1978.

Chapman, D. C., Setting the Scales of the Ocean Response to Isolated Convection, J. Phys. Oceanogr., 28, 606-620, 1998.

Chapman, D. C. and G. Gawarkiewicz, Shallow Convection and Buoyancy Equilibration in an Idealized Coastal Polynya, J. Phys. Oceanogr., 27(4), 555-566, 1997.

Condie, S. A. and P. B. Rhines, Topographic Hadley Cells, J. Fluid Mech., 280, 349-368, 1994.

Emanuel, K. A., Atmospheric Convection, Oxford University Press, New York, 1994.

Fernando, H. J. S., R-R. Chen and D. L. Boyer, Effcts of Rotation on Convective Turbulance, J. Fluid Mech., 228, 513-547, 1991.

Hedstrom, K., SPEM 3.0 User's Guide, Inst. for Naval Oceanography, Technical Report SR-1, 1994.

Lentz, S. J., Sensitivity of the Inner-Shelf Circulation to the Eddy Viscosity Profile, J. Phys. Oceanogr., 25, 19-28, 1995.

Maxworthy, T. and S. Narimousa, Unsteady, Turbulent Convection into a Homogenous, Rotating Fluid with Oceanographic Applications, J. Phys. Oceanogr., $24,865-887,1994$.

Mellor, G. L. and T. Yamada, Devolopment of a Turbulance Closure Model for Geophysical Fluid Problems, Rev. Geophys., 20, 851-875, 1982. 
Mountain, D. G., G. A. Stout and R. C. Beardsley, Surface Heat Flux in the Gulf of Maine, Deep Sea Res., 43(II), 1533-1546, 1996.

Nunes Vas, R. A. and J. H. Simpson, Turbulence Closure Modeling of Esturine Stratification, J. Geophys. Res., 99(C8), 16143-16160, 1994.

Pacanowski, R. C. and S. G. H. Philander, Parameterization of Vertical Mixing in Numerical Models of Tropical Oceans, J. Phys. Oceanogr., 11, 1443-1451, 1981.

Song, Y. and D. Haidvogel, A Semi-Implicit Ocean Circulation Model Using a Generalized Topography-Following Coordinate System, J. Comp. Phys., 115, 228244, 1994.

Stull, R. B., An Introduction to Boundary Layer Meteorology, Kluwer Academic Publishers, Boston, 1988. 


\title{
Chapter 3
}

\section{The Role of Cross-Shelf Eddy \\ Transport in the Steady State}

\section{Response of a Wind-Free Coastal}

\section{Ocean to Winter Time Cooling}

\begin{abstract}
The steady state cross-shelf density gradient of a wind-free coastal ocean undergoing winter time cooling is found for geometries and cooling distributions which do not vary in the alongshore direction. To do this, a relation is found between the gradient, in the depth averaged density and the cross-shelf transport of heat by eddies in the flow field. This scaling is also applied to the cross-shelf transport of a passive tracer by eddies and to the transient response of an ocean being cooled only near the shore. The scaling differs from those of Visbeck, Marshall and Jones [1996], and Chapman and Gawarkiewicz [1997] because it is a turbulent mixing scaling, not an eddy propagation model. The regimes in which Chapman and Gawarkiewicz [1997] and the scalings below are valid are discussed. The scalings are compared favorably to the results of a primitive equation numerical model.
\end{abstract}




\begin{tabular}{|c|c|c|}
\hline \multicolumn{3}{|c|}{ Table of Symbols } \\
\hline SYMBOL & DESCRIPTION & DEFINED BY \\
\hline & horizontal diffusivity of density or tracer & eq. (3.46) and (3.38) \\
\hline$c$ & concentration of passive tracer & eq. (3.46) \\
\hline $\mathcal{C}$ & fit between scaling and numerical model & eq. (3.25) \\
\hline$F$ & $\begin{array}{l}\text { cross-shelf depth integrated density flux, } \\
F=\int_{-h}^{0} v \rho d z \text { averaged in the along-shelf } \\
\text { direction }\end{array}$ & eq. $(3.4)$ \\
\hline$F_{0}$ & $\begin{array}{l}\text { cross-shelf depth integrated density flux at } \\
\text { ocean boundary }\end{array}$ & eq. (3.6) \\
\hline$F_{c}$ & depth integrated cross-shelf flux of tracer & eq. (3.45) \\
\hline$h$ & water depth & \\
\hline$L_{d}$ & first internal radius of deformation & \\
\hline$L_{R h}$ & Rhines arrest scale & eq. (3.13) \\
\hline$L_{f r}$ & frictional arrest scale & eq. (3.19) \\
\hline$L^{*}$ & cross-shelf length scale of eddies & eq. $(3.20)$ \\
\hline$L^{\prime}$ & numerical model length scale divided by $L^{*}$ & eq. (3.33) \\
\hline $\mathcal{L}$ & $L_{R h} / L_{f r}$ & eq. (3.21) \\
\hline$\overline{\text { overbar }}$ & alongshore and depth average operator & \\
\hline$Q$ & surface density flux, $\mathrm{kg} \mathrm{m}^{-2} \mathrm{~s}$ & \\
\hline$r$ & bottom friction in linear drag law $\vec{\tau}=\rho_{0} r \vec{u}$ & eq. $(3.16)$ \\
\hline $\mathcal{T}_{\text {advec }}$ & advective time scale & eq. (3.15) \\
\hline $\mathcal{T}_{\text {diff }}$ & diffusive time scale & eq. (3.48) \\
\hline $\mathcal{T}_{f r}$ & frictional time scale & eq. $(3.16)$ \\
\hline$x$ & alongshelf direction & figure 3.1 \\
\hline$y$ & cross-shelf direction & figure 3.1 \\
\hline$y_{\text {cool }}$ & offshore limit of cooling & eq. (3.7) \\
\hline$y_{0}$ & offshore boundary location & figure 3.1 \\
\hline$V^{*}$ & velocity scale & eq. (3.12) \\
\hline$V^{\prime}$ & $\begin{array}{l}\text { numerical model velocity divided by veloc- } \\
\text { ity scale }\end{array}$ & eq. $(3.31)$ \\
\hline$\alpha$ & negative of bottom slope & eq. (3.7) \\
\hline$\beta$ & $\begin{array}{l}\text { topographic } Q G \text { potential vorticity } \\
\text { gradient }\end{array}$ & eq. (3.14) \\
\hline$\gamma$ & $\begin{array}{l}\text { correlation between depth averaged den- } \\
\text { sity and depth averaged cross-shelf velocity }\end{array}$ & eq. (3.11) \\
\hline$\overline{\rho_{y}}$ & $\begin{array}{l}\text { alongshore average of cross-shelf gradient } \\
\text { of depth averaged density }\end{array}$ & \\
\hline
\end{tabular}




\subsection{Introduction}

An ice-free coastal ocean in the winter will be cooled over length scales large compared to the shelf width or an internal radius of deformation of the coastal ocean. The shallow waters become colder than the deeper waters, thus causing a crossshelf density gradient. Because of rotation, this temperature gradient forces a mean alongshore flow which is unable to drive a cross-shelf heat flux strong enough to balance the surface cooling. A cross-shelf heat flux that balances the cooling can, however, be driven by eddies in the mean alongshore flow.

This situation is very similar to that found in the atmosphere, where meridional gradients in solar heating are balanced by meridional heat fluxes driven by instabilities in the mean zonal flow. Green [1970] and Stone [1972] developed parameterizations based on various eddy closure schemes for the instability-driven meridional transport of heat in the atmosphere. The work of Green [1970] has been extended to isolated cooling in the deep ocean [Visbeck, Marshall and Jones, 1996] and the coastal ocean [Chapman, 1998; Chapman and Gawarkiewicz, 1997]. The dynamics responsible for the success of the scalings in the oceanic papers has been explained by Spall and Chapman [1998]. They attribute the heat flux to the self advection of isolated eddy pairs away from the cooling region.

The work below analyzes the case in which the cross-shelf heat flux is driven by eddies which are not isolated, but instead interact and form a turbulent flow field which cascades to larger scales. The eddies are forced to interact because the large spatial scales of cooling create baroclinically unstable density fields over the entire shelf, so that eddies are generated everywhere across the shelf and are unable to escape the shelf without interacting. Furthermore, even when the cooling is limited to a small portion of the shelf, bottom friction inhibits the cross-shelf propagation of eddies, again forcing the eddies to interact.

First, a representative numerical model run with a bottom slope and cooling similar to the Mid-Atlantic Bight will be presented in order to provide context for 
the following scaling arguments.

Next, a scaling for the cross-shelf density gradient needed to drive a cross-shelf heat flux sufficient to balance the surface cooling and maintain a statistically steady state flow field will be presented. This is the heart of this work: all else is an elaboration on this theme.

This scaling will then be tested in numerical models. Once it has been found sufficient, the scaling will be used to devise a simple model of the transient response of a wind-free coastal ocean to surface cooling, under the assumption that the time scales of change for the density field are long enough to consider the response of the ocean to be quasi-steady. This simple model is then tested and compared to the ideas of Chapman and Gawarkiewicz [1997] and Spall and Chapman [1998].

The simple model is then used to describe the transport of a passive tracer in an eddy-filled coastal ocean.

\subsection{Demonstration}

Before deriving scalings for the steady state density structure for a continental shelf exposed to winter time cooling, it is useful to choose one geometry and one surface heat flux distribution and examine in a numerical model the evolution of a coastal ocean from a homogeneous state to one with a steady state cross-shelf density gradient. The geometry chosen has a linear bottom slope of $10^{-3}$ between a depth of 10 $\mathrm{m}$ and a $170 \mathrm{~m}$, and is meant to model crudely the northeast coast of North America [Brown and Beardsley, 1978; Mountain, Stout and Beardsley, 1996]. This geometry is illustrated in figure 3.1. The cooling is modeled with a density flux of $7 \times 10^{-6}$ $\mathrm{kg} \mathrm{m}^{-2} \mathrm{~s}^{-1}$, which is equivalent to a heat loss of $300 \mathrm{~W} \mathrm{~m}^{-2}$ from $3^{\circ} \mathrm{C}$ water or 170 $\mathrm{W} \mathrm{m}^{-2}$ from $10^{\circ} \mathrm{C}$ water. (The details of this conversion are given in the appendix.) This cooling is also meant to be typical of the northeast coast of North America. The model used is SPEM 5.1 [Hedstrom, 1994], a primitive equation, modified sigma 


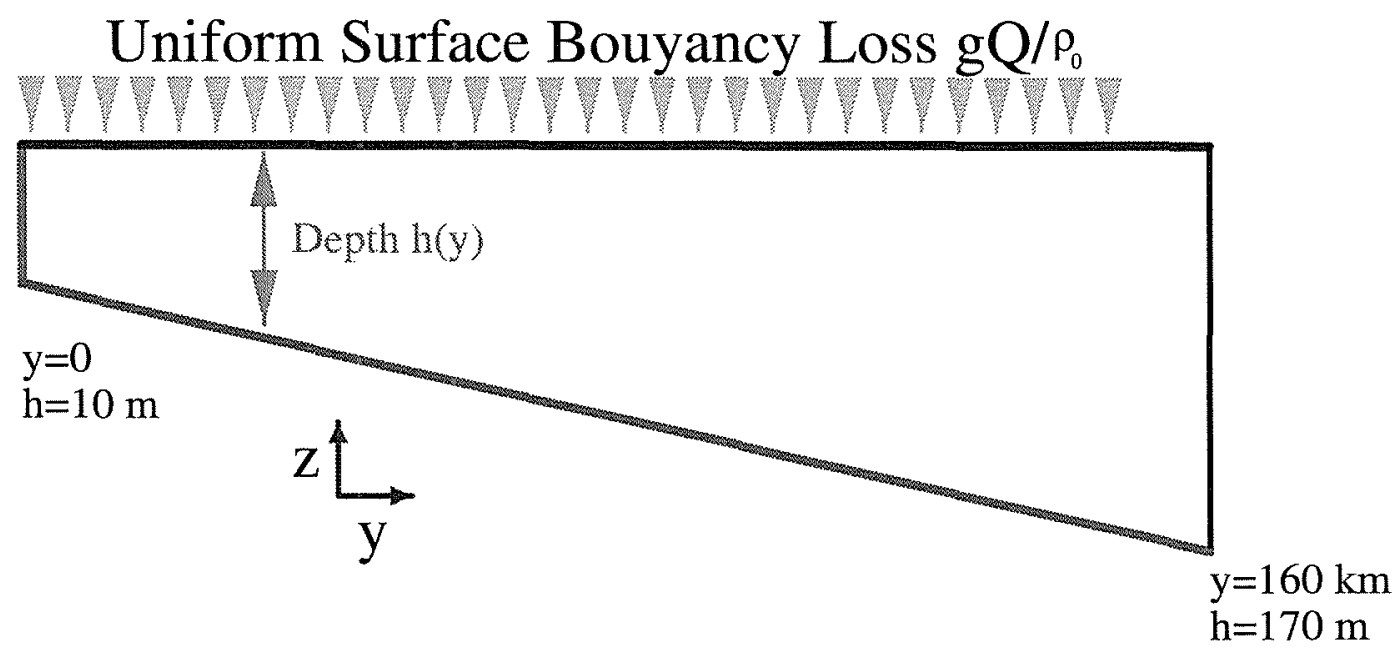

Figure 3.1: The geometry and forcing of the model described in section 3.2.

coordinate, hydrostatic model. The model is run in an alongshelf periodic domain with walls at the coast and at the "offshore" boundary (runs are made later with an open offshore boundary). The vertical walls have free slip boundary conditions. The details of the model are given in the appendix.

When cooling is imposed on the initially homogeneous water of the model, convection initially mixes the water from top to bottom in less then an inertial period $\left(2 \pi f^{-1}\right)$, and keeps the entire water column slightly unstably stratified. This strong vertical mixing inhibits the cross-shelf heat flux while preventing the earth's rotation from affecting the flow strongly. During this regime, the cross-shelf heat transport is small, the heat balance is essentially one dimensional, and the density evolves as

$$
\begin{aligned}
\frac{\partial \rho}{\partial t} & =\frac{Q}{h} \\
\rho & =\frac{Q t}{h} .
\end{aligned}
$$

Since there is a bottom slope, there is a cross-shelf density gradient of

$$
\frac{\partial \rho}{\partial y}=-\frac{Q t}{h^{2}} \frac{\partial h}{\partial y}
$$


which drives a weak $\left(<1 \mathrm{~cm} \mathrm{~s}^{-1}\right)$ ageostrophic cross-shelf flow. The top panels of figure 3.2 shows the density field in the model run during this regime, at $t=40$ days.

Chapter 2 shows that the cross-shelf flow $v$ driven by the cross-shelf density gradient forces the water column to become stably stratified near the bottom when the cross-shelf flow near the bottom exceeds

$$
\left.v\right|_{z=-h} \geq \frac{h}{t}\left(\frac{\partial h}{\partial y}\right)^{-1}
$$

This first occurs in this run at the coastal edge after 40 days of cooling. Once the cross-shelf flow stabilizes the water column, convection no longer reaches the bottom. The stable stratification reduces mixing at the base of the water column and allows rotation to affect the dynamics, allowing baroclinic instabilities to form. The onset of the instabilities is illustrated in the middle panel of figure 3.2.

Once instabilities in the flow form, they quickly start to transport heat across the shelf, reducing the cross-shelf density gradient by $40 \%$ in 15 days (fig 3.3). In another week, the cross-shelf density gradient achieves a statistical steady state in which surface cooling is balanced by a cross-shelf heat flux driven by the cross-shelf density gradient. It is for this steady state that the scaling below solves. Because there is a wall for the "offshore" boundary, and the surface is everywhere cooled, the mean density of the domain always increases. However, it increases everywhere at the same rate, so the mean cross-shelf density gradient does not change. The time evolution of the mean cross-shelf density gradient is plotted in figure 3.3 and the density field for the steady state density gradient is shown in the bottom panels of figure 3.2 .

The cross-shelf heat flux which leads to the statistical steady state in the crossshelf density field is caused by turbulent mixing driven by a dense field of eddies. This eddy field is illustrated in figure 3.4. The eddies seen in this picture are transient, appearing only to be sheared apart in a few days. No consistent cross- 

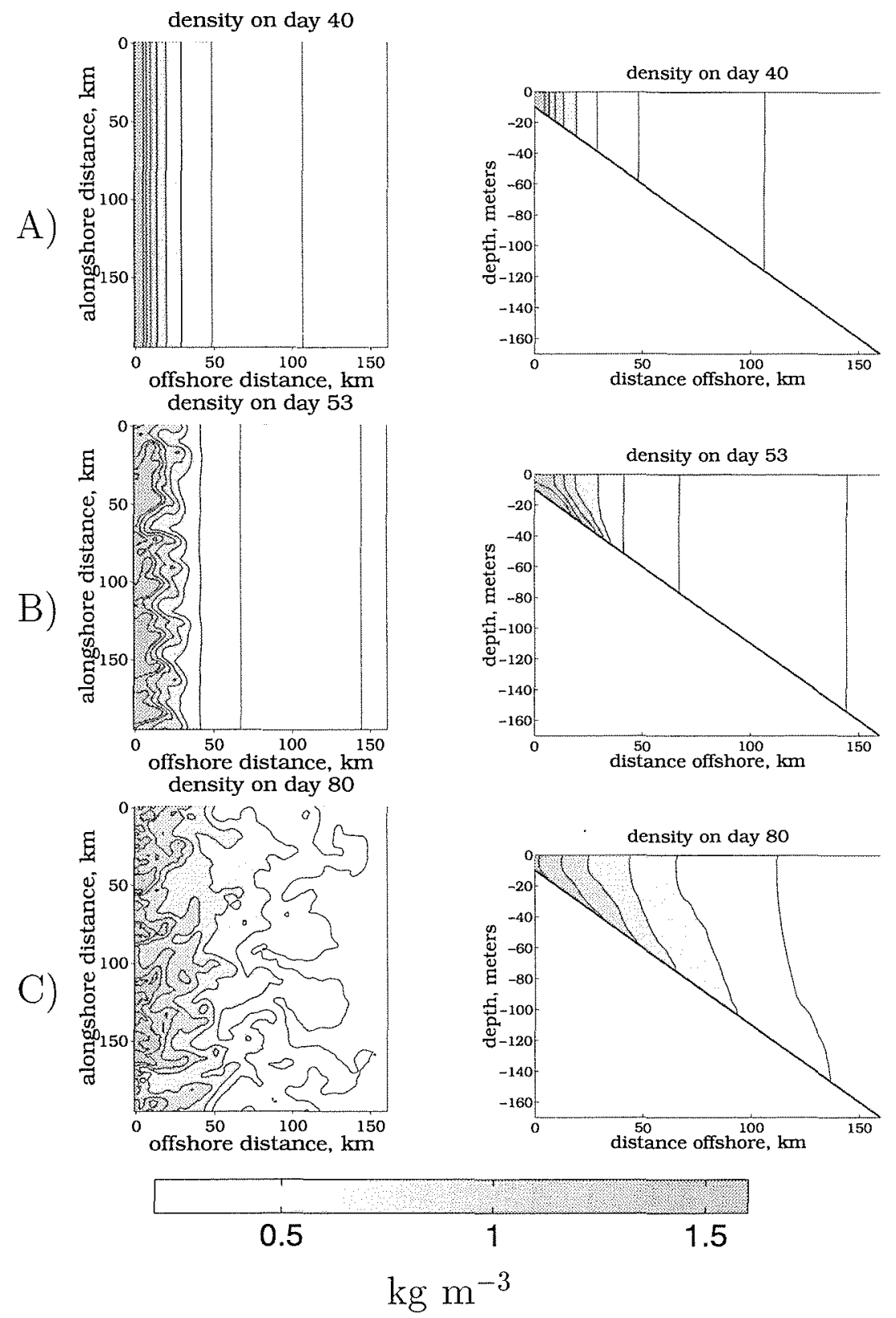

Figure 3.2: The lefthand plots are of the depth averaged density, the righthand of the alongshelf averaged density. Panel $\mathrm{A}$ is from 40 days after the start of cooling, panel B after 53 days, and panel $\mathrm{C}$ after 80 days. 


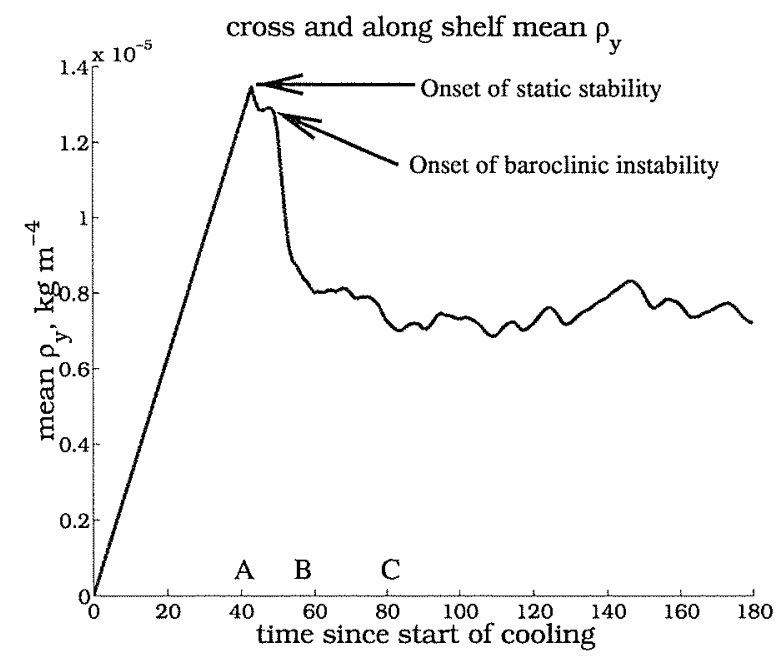

Figure 3.3: The cross-shelf averaged cross-shelf gradient in the depth and alongshelf averaged density. The average is computed across the entire model domain. The letters on the abscissa refer to the panels of figure 3.2 .

shelf eddy propagation is seen in the model.

It is not only the cross-shelf density gradient that reaches steady state. The mean velocities, the length scales of the eddies, and the variance of the velocities all reach statistically steady states. The flow at this point appears turbulent in the sense that the flow at a point is unpredictable (i.e. decorrelates after a finite time).

Another route to this steady state, which may be more likely in reality, is a cessation of cooling for a short time any time after the density gradient predicted by the one dimensional model becomes greater than the eventual steady state cross-shelf density gradient (day 25 in this case). While the cooling is halted, the instabilities which transport heat across the shelf can spin up, providing the mechanism to maintain the steady state once the cooling resumes. 


\section{Surface Currents}

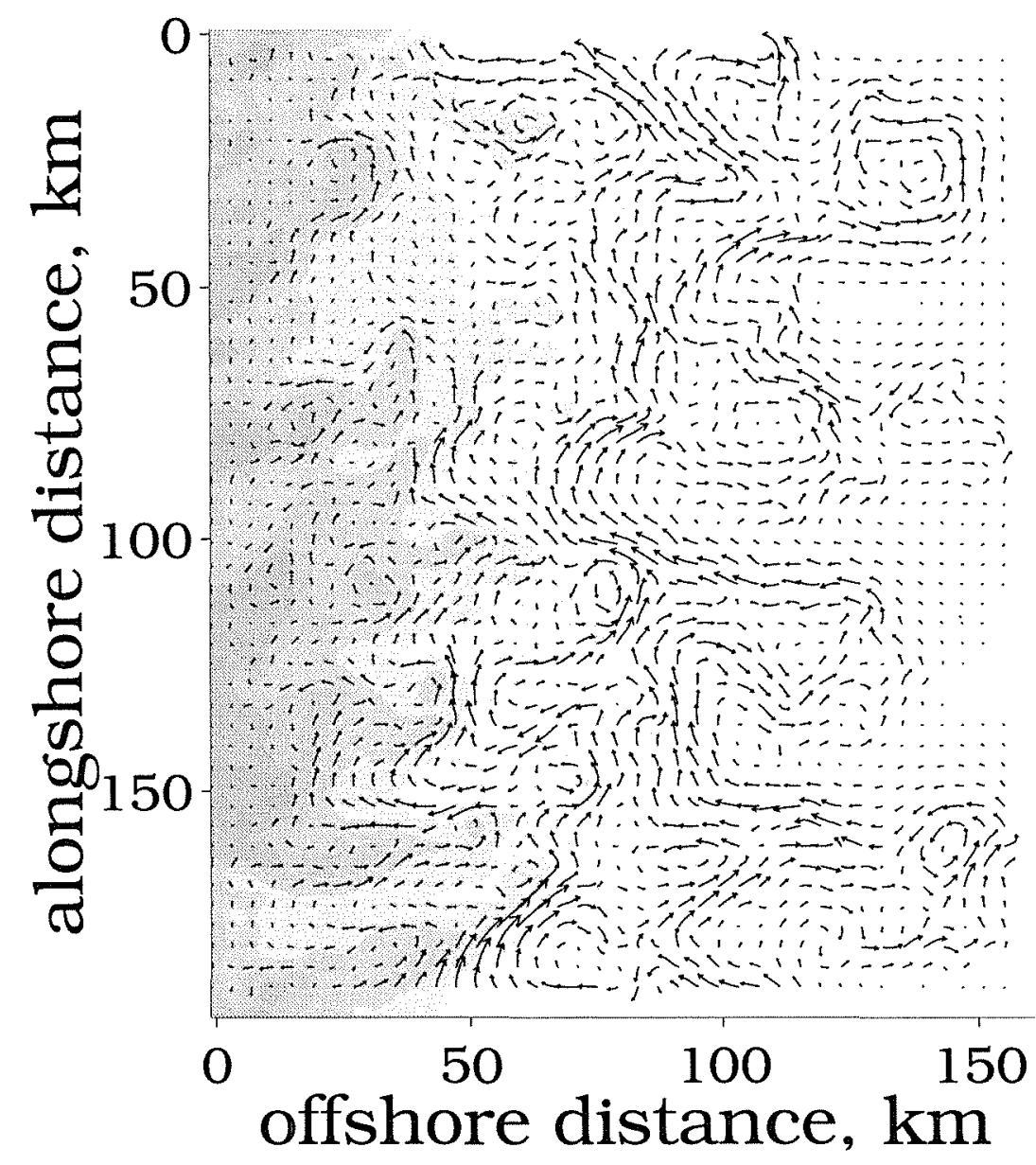

Figure 3.4: The depth averaged density from panel $\mathrm{C}$ of figure 3.2 (gray shading) overlaid by the surface current vectors. A $1 \mathrm{~m} \mathrm{~s}^{-1}$ flow would produce a $20 \mathrm{~km}$ arrow. The maximum current in this plot is $0.47 \mathrm{~m} \mathrm{~s}^{-1}$. 


\subsection{Outline of the Scaling for the Steady State $\partial \bar{\rho} / \partial y$}

In the preceding section, numerical model results were shown that described the evolution of a coastal ocean from a homogeneous body of water to a stratified ocean with a statistically steady cross-shelf density gradient. This density gradient was forced by a surface heat loss, and the density gradient reached steady state when it drove a cross-shelf heat flux sufficient to maintain that steady state.

The scalings below solve for that cross-shelf density gradient in two steps. First, given the distribution of surface cooling and assuming the heat flux at the shelf break is known, the cross-shelf heat flux for a steady state temperature (and hence density) gradient is diagnosed. Assuming that the cross-shelf heat flux at the seaward side of the domain is known is clearly artificial, but making that assumption allows one to focus on the processes occurring on the shelf. It is important to note that unless the cross-shelf heat flux at the oceanward boundary exactly matches the surface cooling, the mean density of the water over the shelf changes with time. This in no way precludes the existence of a statistically steady cross-shelf density gradient or a statistically steady flow field. It is also assumed that the geometry, surface cooling, and heat flux at the shelf break do not vary in time or the alongshelf direction.

Once the cross-shelf heat (and thus density) flux has been diagnosed, it can be converted to a mean cross-shelf temperature (density) gradient by finding a relation between the cross-shelf heat flux and the cross-shelf density gradient that drives it. Thus the second step in deriving the cross-shelf temperature (density) gradient is

finding that scaling which relates the cross-shelf density gradient to the cross-shelf flux it forces. 


\subsection{Buoyancy/Heat Balance}

The derivation below solves for the cross-shelf flux of density which leaves the mean flow fields in a statistical steady state even as the water is being cooled by the atmosphere. For the currents to be in a statistical steady state, the cross-shelf density gradient must also be in a statistical steady state. For the cross-shelf density gradient to be in a steady state, the time rate of change of density must be the same everywhere on the shelf. Thus the derivation below solves for the cross-shelf density flux for which the time rate of change of density, $\partial \rho / \partial t$, is a constant across the shelf. Density is used instead of temperature because it is the density gradient, not the temperature gradient, which drives the flow fields. The derivation below assumes a linear equation of state, which, while a rather cavalier assumption in near-freezing water, greatly simplifies the analysis of this idealized problem.

The assumption of a linear equation of state allows one to write the conservation of buoyancy and volume as

$$
\frac{\partial \bar{\rho}}{\partial t}+\frac{1}{h} \frac{\partial F}{\partial y}=\frac{Q}{h}
$$

where the overbar is a depth and alongshore averaging operator and $F$ is the depth integrated cross-shelf heat flux

$$
F \equiv h \overline{v \rho}
$$

The density flux at the seaward edge of the shelf, $F_{0}=F\left(y_{0}\right)$, is assumed to be known, and the flux at the coast must be zero. Since $\partial \bar{\rho} / \partial t$ must be the same 
everywhere, (3.4) can be solved for

$$
\begin{aligned}
\frac{\partial \bar{\rho}}{\partial t} & =\frac{\int_{0}^{y_{0}} Q(y) d y-F_{0}}{,} \int_{0}^{y_{0}} h(y) d y \\
F & =\int_{0}^{y} Q(y) d y-\frac{\partial \bar{\rho}}{\partial t} \int_{0}^{y} h(y) d y .
\end{aligned}
$$

To illustrate the solution to (3.4), (3.6), three sets of bathymetry and cooling are examined below and in figure 3.5 , for both the case when $F_{0}=0$ and when $F_{0}$ balances the surface forcing. All three cases are subsets of

$$
\begin{aligned}
& h=\alpha y+h_{0}, \\
& Q=\left\{\begin{array}{ll}
Q_{0} & \text { for } y \leq y_{\text {cool }} \\
0 & \text { for } y>y_{\text {cool }}
\end{array} .\right.
\end{aligned}
$$

If the offshore lateral flux of density balances the surface cooling so there is no net cooling of the water, e.g. $F_{0}=Q y_{\text {cool }}$, the steady state $F$ for this geometry is

$$
F=\left\{\begin{array}{ll}
Q_{0} y & \text { for } y \leq y_{\text {cool }} \\
Q_{0} y_{\text {cool }} & \text { for } y>y_{\text {cool }}
\end{array} .\right.
$$

If there is no heat flux from the ocean $\left(F_{0}=0\right)$, the steady state $F$ for this geometry is

$$
F=\left\{\begin{array}{cl}
Q_{0} y-Q_{0} y_{\text {cool }} \frac{0.5 \alpha y^{2}+h_{0} y}{0.5 \alpha y_{0}^{2}+h_{0} y_{0}} & \text { for } y \leq y_{\text {cool }} \\
Q_{0} y_{\text {cool }}-Q_{0} y_{\text {cool }} \frac{0.5 \alpha y^{2}+h_{0} y}{0.5 \alpha y_{0}^{2}+h_{0} y_{0}} & \text { for } y>y_{\text {cool }}
\end{array} .\right.
$$

It is important to note that the existence of a non-zero cross-shelf heat flux does not depend on the existence of a cross-shelf gradient in the surface heat flux or bottom depth, but can be driven by the oceanic boundary condition. 


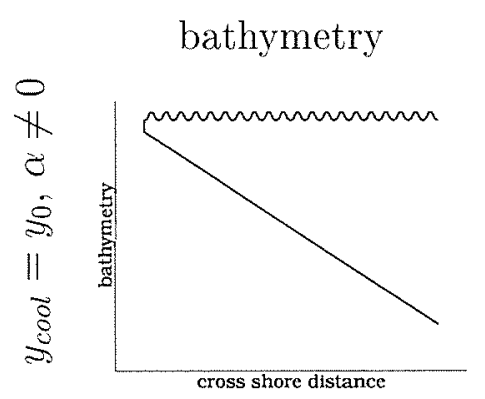

surface cooling depth integrated density flux
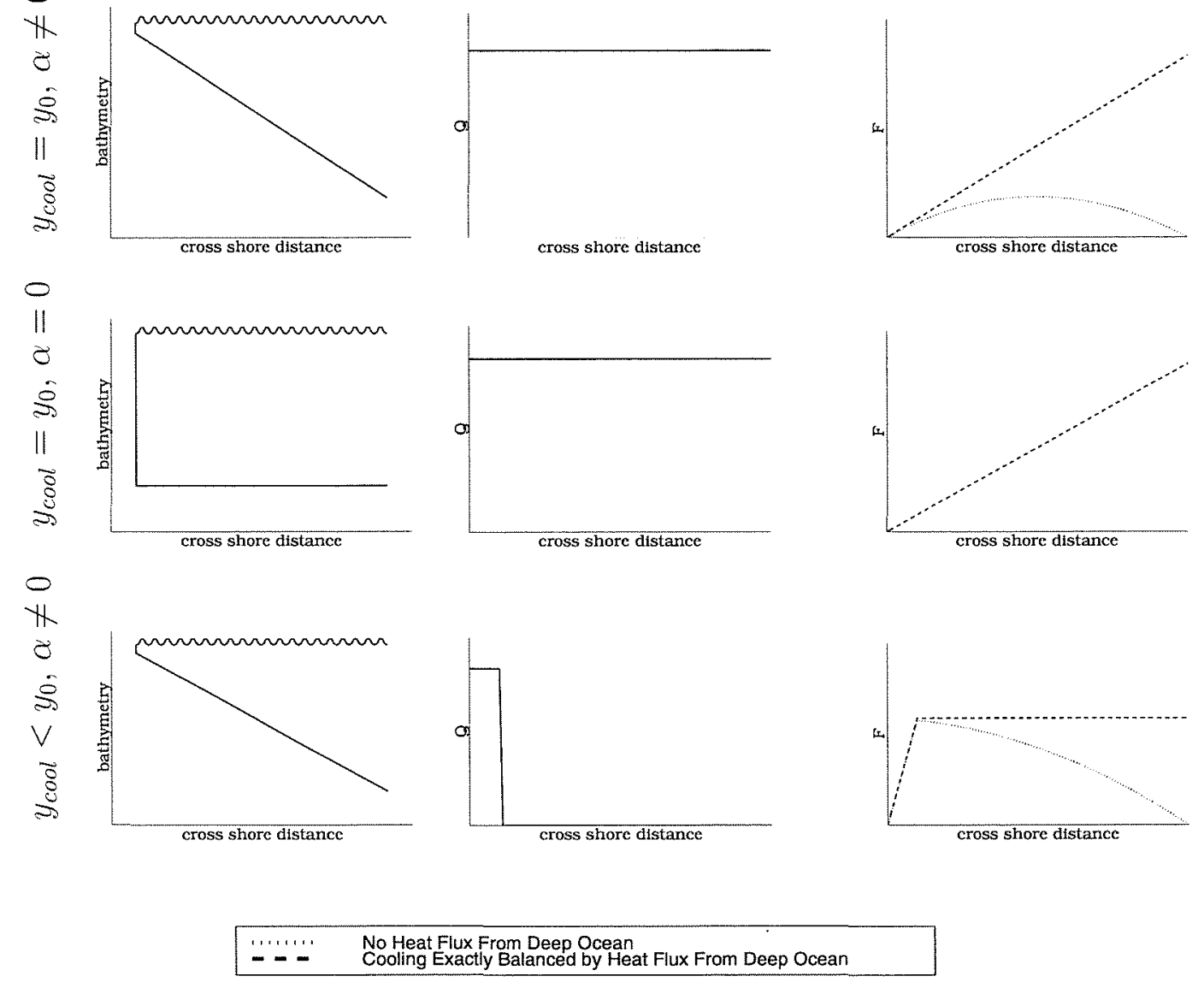

Figure 3.5: The cross-shelf, depth-integrated density flux for three different cooling distributions and geometry. The top panel is for $\alpha \neq 0, y_{\text {cool }}=y_{0}$, the middle panel for $\alpha=0, y_{\text {cool }}=y_{0}$, and the bottom panel is for $\alpha=0, y_{\text {cool }}<y_{0}$. 


\subsection{Scaling $\partial \bar{\rho} / \partial y$ from $F$}

In the previous section, the cross-shelf depth-integrated density flux which leaves the flow fields and density gradients in a statistical steady state were found. In this section the relation between the cross-shelf depth-integrated density flux and the gradient in the depth-mean density is obtained, and then that relation is inverted for the gradient of the depth-mean density implied by the surface cooling.

The cross-shelf depth integrated density flux $F$ can be written

$$
F=h \overline{v \rho} .
$$

This quantity can be scaled as

$$
\begin{aligned}
F & =h \gamma V^{*} \rho^{*}, \\
\rho^{*} & =\overline{\rho_{y}} L^{*},
\end{aligned}
$$

where $V^{*}$ is a cross-shelf velocity scale, $\rho^{*}$ is a density anomaly scale, $\overline{\rho_{y}}$ is the alongshore averaged cross-shelf gradient in the depth-mean density, $L^{*}$ is the crossshelf length scale of the eddies which transport heat across the shelf, and $\gamma$ is the correlation between the cross-shelf velocity and the density field. $\gamma$ will not be used as a fitting parameter in this work. This scale for the cross-shelf flux assumes that the flux is the product of the depth averaged velocity times the depth average density anomaly and that the transport carried by the mean overturning circulation is small. The scale is a classical turbulent mixing scale, which makes the strong assumption that there is no transport of density in the absence of a mean density gradient. A good examination of the restrictions on a flow field needed for this last approximation to be valid is given in Davis [1987].

The scalings below for $V^{*}$ and $L^{*}$ will make a further limiting assumption: they will be based on the local cross-shelf density gradient. It is possible to conceive of a 
turbulent mixing scale with the form of (3.11) where the eddies represented by $V^{*}$ and $L^{*}$ are remotely forced. There are circumstances where this must be correct, e.g. near a jet radiating eddies. Nonetheless, I shall neglect this case.

\subsubsection{The Velocity Scale $V^{*}$}

The velocity scale is

$$
V^{*}=\frac{g h}{\rho_{0} f} \overline{\rho_{y}}
$$

which is an assumption that the cross-shelf velocity perturbation scales as the mean alongshelf thermal wind velocity, and that the bottom Ekman layer occupies a negligible fraction of the water column. This assumption is traditionally justified by invoking an equipartition of kinetic and potential energy in the instability, which is the same as assuming that the length scale of the instabilities is the radius of deformation [Pedlosky, 1987 p. 374 , VMJ]. Since, in general, the length scales found below are not the radius of deformation, this argument is not very convincing in this case. There is some hope in deriving a rigorous upper bound on $V^{*}$ by the method of Shepherd [1988] but with a more realistic dissipation scheme (c.f. Held and Larichev [1995]). As of now, however, the scale must be regarded as an ad hoc assumption with a good pedigree [Chapman, 1998; Chapman and Gawarkiewicz, 1997; Stone, 1972, VMJ].

\subsubsection{The Correlation Between $v$ and $\rho: \gamma$}

The theoretical basis for choosing a correlation between the crossshelf velocity and the density perturbation is currently limited to linear instability theory. It is unclear what relevance linear instability theory has to finite amplitude eddies. However, it is not unreasonable to expect the linear instability analysis to capture approximately the dynamics of the eddies, and several authors have gone so far as to base eddy 
turbulence closure schemes on the linear instability solutions [Killworth, 1998; Stone, 1972]. Blumsack and Gierasch [1972] have performed a linear stability analysis for an Eady instability over a sloping bottom, and found a correlation coefficient $\gamma$ which varies from $\approx 0.4$ for a flat bottom to $\approx 0.7$ for a bottom slope half that of the isopyncnal slope.

\subsubsection{The Cross-Shelf Length Scale: $L^{*}$}

It is in its particular specification of a cross-shelf (meridional) length scale that this work differs most significantly from previous coastal [Chapman and Gawarkiewicz, 1997], oceanic [Killworth, 1998, VMJ] and atmospheric [Green, 1970; Stone, 1972] work. Simply choosing the radius of deformation or the wavelength of the most unstable mode ignores much work that shows a cascade to larger horizontal and vertical scales in unstable geostrophic flows. Rhines [1977], Held and Larichev [1995], and LaCasce [1996] show that in flows where the internal radius of deformation $L_{d}$ is less than the Rhines arrest scale $L_{R h}$, a turbulent flow will experience a cascade to larger horizontal and vertical scales until the cross-shelf length scale is the Rhines arrest scale

$$
L_{R h}=\sqrt{\frac{2 V^{*}}{\beta}} .
$$

This scale is most easily understood as the scale at which advective terms in the quasigeostrophic potential vorticity equations becomes smaller than the topographic $\beta$ term where

$$
\beta=\frac{f}{h} \frac{\partial h}{\partial y}
$$

Once the scales of flow have increased to $L_{R h}$, linear terms dominate the non-linear terms, thus preventing a further cascade to large scales [Pedlosky, 1987, p. 174]. 
Thus one candidate for the cross-shelf length scale of the eddies which transport heat is $L_{R h}$.

However, the time scale of the cascade of energy to larger scales, and the time scale for the conversion of potential to kinetic energy, is

$$
\mathcal{T}_{\text {advect }}=\frac{L^{*}}{V^{*}}
$$

[Pedlosky, 1987, p. 174]. The time scale for the dissipation by bottom friction of barotropic features, and baroclinic features where $L^{*} \gg L_{D}$, is

$$
\mathcal{T}_{\text {fric }}=\frac{h}{r},
$$

where $r$ is the friction coefficient for a linear drag law of the form $\vec{\tau}=\rho_{0} r \vec{u}[S t$ Maurice and Veronis, 1975]. If $\mathcal{T}_{\text {advec }}>\mathcal{T}_{\text {fric }}$, the energy at the largest scales would be dissipated by bottom friction faster than energy could cascade to that scale. If the choice of $L^{*}=L_{R h}$ caused $\mathcal{T}_{\text {advec }}>\mathcal{T}_{\text {fric }}$ it is improbable that $L_{R h}$ be the length scale, and instead it seems probable that the energy-containing scale would be the one for which the conversion of potential energy to kinetic would be balanced by the dissipation of energy by friction, e.g.

$$
\mathcal{T}_{\text {advec }}=\mathcal{T}_{\text {fric }}
$$

or

$$
\frac{L}{V}=\frac{h}{r},
$$

so

$$
L_{f r}=\frac{h}{r} V^{*} .
$$


$L^{*}$ can thus have one of two values,

$$
L^{*}= \begin{cases}L_{R h} & \text { if } \mathcal{L}<1 \\ L_{f r} & \text { if } \quad \mathcal{L}>1\end{cases}
$$

where

$$
\mathcal{L}=\frac{L_{R h}}{L_{f r}}
$$

A problem with the derivation of $L_{\text {fric }}$ is the assumption that the flow is close to barotropic - e.g. that $V^{*}$ is the appropriate scale to use for the bottom stress estimate. In favor of this approximation is the fact that, as the flow cascades to larger scales, $L^{*} \gg L_{D}$ and the flow becomes more barotropic. This problem is re-addressed in section 3.6.3.

The derivation of $L_{R h}$ begs the question of the fate of the energy that has cascaded to that scale. In the barotropic numerical experiments of Rhines [1977], and in the later baroclinic work of Held and Larichev [1995] and LaCasce [1996], the flow forms zonal (alongshore) barotropic jets once the cascade has occurred. Here it is assumed that the jets will grow in strength till their dissipation through bottom friction matches the cascade of energy to the jets. In the numerical runs discussed below, this seems to be the case - however, if the friction is small enough, the relative vorticity of the jets dominates the planetary vorticity, and so $L_{R h}$ becomes irrelevant. In the real ocean, this point is likely moot since the shelf and slope is a waveguide, and the energy which cascades to $L_{R h}$ can propagate down the coast as coastal trapped waves away from the cooling region. 


\subsubsection{Given $V^{*}$ and $L^{*}$, what are $F, \overline{\rho_{y}}$ and $\mathcal{L}$ ?}

The scales for $V^{*}$ and $L^{*}$ can be substituted into (3.11) to obtain an estimate of $F$ :

$$
F=\left\{\begin{array}{ll}
r^{-1} \frac{\gamma g^{2} h^{4}}{\rho_{0}^{2} f^{2}} \bar{\rho}_{y}^{3} & \text { if } L=L_{f r}, \text { i.e. } \mathcal{L} \geq 1 \\
2^{\frac{1}{2}} \frac{\partial h^{-\frac{1}{2}}}{\partial y} \frac{\gamma h^{3} g^{\frac{3}{2}}}{\rho_{0}^{\frac{3}{2}} f^{2}} \bar{\rho}_{y}^{\frac{5}{2}} & \text { if } L=L_{R h}, \text { i.e. } \mathcal{L} \leq 1
\end{array} .\right.
$$

These can be solved for $\overline{\rho_{y}}$

$$
\overline{\rho_{y}}= \begin{cases}r^{\frac{1}{3}} \frac{\rho_{0}^{\frac{2}{3}} f^{\frac{2}{3}}}{\gamma^{\frac{1}{3}} h^{\frac{4}{3}} g^{\frac{2}{3}}} F^{\frac{1}{3}} & \text { if } \mathcal{L} \geq 1 \\ \frac{\partial h^{\frac{1}{5}}}{\partial y} \frac{\rho_{0}^{\frac{3}{5}} f^{\frac{4}{5}}}{2^{\frac{1}{5}} \gamma^{\frac{2}{5}} h^{\frac{6}{5}} g^{\frac{3}{5}}} F^{\frac{2}{5}} & \text { if } \mathcal{L} \leq 1\end{cases}
$$

which can be substituted into $(3.21)$ to get $\mathcal{L}$

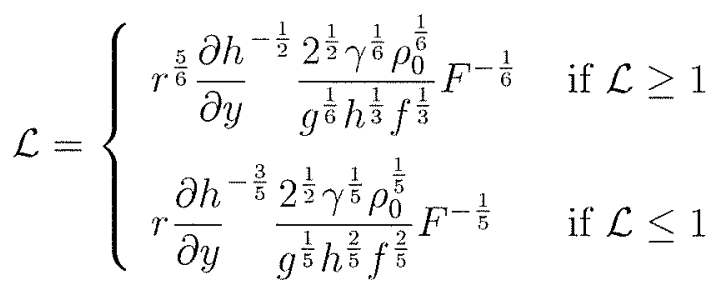

(Since both estimates of $\mathcal{L}$ are equal to one at the same time, this is mathematically consistent.) These scales can be combined with (3.6) to find $\overline{\rho_{y}}$ and $\mathcal{L}$ for a given bathymetry and cooling distribution.

Some implications of these scales are:

- Bottom friction does not affect the solution when $\mathcal{L} \leq 1$ (but see section 3.6.4, "Where the Scalings Fail and Why").

- Bottom slope does not affect the solution when $\mathcal{L} \geq 1$ (but see section 3.6.4, "Where the Scalings Fail and Why").

- The steady state cross-shelf density gradient is only weakly dependent on the 
cooling rate.

\subsection{Testing the Scales in a Numerical Model}

The scalings derived above are tested in a series of numerical model experiments for the geometry given in (3.7). The models are run until they reach a statistical steady state, and then they are run for 50-100 days more to reduce the errors in the averaged quantities. The model is defined as being in a steady state when the RMS cross-shelf velocities, the mean eddy length scales, and the mean cross-shelf density gradients no longer evolve in time.

The scales for the density gradient are first tested for a flat bottom $(\mathcal{L}=\infty)$, and then the transition from the frictional length scale $(\mathcal{L}>1)$ to the Rhines arrest scale $(\mathcal{L}<1)$ is examined. The limits of validity of the scaling are then discussed, and the scales for the velocity and length of the eddies are compared to the numerical model.

\subsubsection{Flat Bottom Results}

Over a flat bottom, the Rhines arrest scale is infinite and thus irrelevant. Runs over a flat bottom allow one to check the $\mathcal{L}>1$ solutions without being concerned about which dynamical regime is relevant. For the runs described below, the cooling is limited to the first $10 \mathrm{~km}$ offshore $\left(y_{\text {cool }}=10 \mathrm{~km}\right)$, and the shelf is $160 \mathrm{~km}$ wide. The offshore boundary has no flux through it $\left(F_{0}=0\right)$. The base case chosen to vary the parameters around has a surface cooling represented by a density flux of $1 \times 10^{-4}$ $\mathrm{kg} \mathrm{m}^{-2} \mathrm{~s}^{-1}$, a depth of $100 \mathrm{~m}$, and a bottom drag of $4.5 \times 10^{-4} \mathrm{~m} \mathrm{~s}^{-1}$. The surface density flux is about twice the base case of Chapman and Gawarkiewicz [1997], as is the water depth. The time it takes for the entire $160 \mathrm{~km}$ wide shelf to reach steady state is 50 to 100 days, a time not short compared to a winter. Thus these models, while useful in examining the scalings, are not likely to be directly relevant to an 
existing ocean. However, the transient response of the models to cooling is likely to be relevant to the arctic, and this response is studied in section 3.7. Section 3.7 also examines the relation between the scalings in (3.23) and the work of Chapman and Gawarkiewicz [1997].

Once the models have reached steady state, eddies inhabit the entire domain, mixing density across the shelf. Figure 3.6 gives the depth averaged density and the transport streamfunction for a day in the base flat-bottom model. The eddies appear, propagate a short distance, and then get sheared apart by other eddies. Even though the forcing is confined to within $10 \mathrm{~km}$ of the coast, the eddies are not generated solely at this region, but can be generated outside this region as well. The lives of four eddies are followed for twelve days in figure 3.7, starting from the day shown in 3.6 .

In figure 3.8, the cross-shelf depth-integrated density flux $F$ from the base case is plotted. $F$ is broken into two parts- $F_{D I}$, which is the flux caused by the depth averaged velocity and depth averaged density field, and $F_{D D}$, which is the flux forced by the depth dependent parts of the velocity and density. The flux in the model is dominated by $F_{D I}$. Since the scaling in section 3.5 assumes that the cross-shelf density flux is driven by the depth average velocity and density fields, it is reassuring that it is so in the model.

The results of the model runs are compared to the scales derived above by fitting the $\partial \bar{\rho} / \partial y$ observed in the model:

$$
\text { Numerical model } \frac{\partial \bar{\rho}}{\partial y}=\mathcal{C} \times\left(\text { Scale } \frac{\partial \bar{\rho}}{\partial y}\right)
$$

The fit is made by computing a scale for the cross-shelf density gradient by integrating 3.23 across the shelf, computing the depth, time and alongshore averaged density from the steady state of the numerical model, de-meaning both, and finding the least squares optimal regression between the two. The fit is done at least one eddy length scale from the walls at $y=0$ and $y=160 \mathrm{~km}$. Error bars on the fit are 


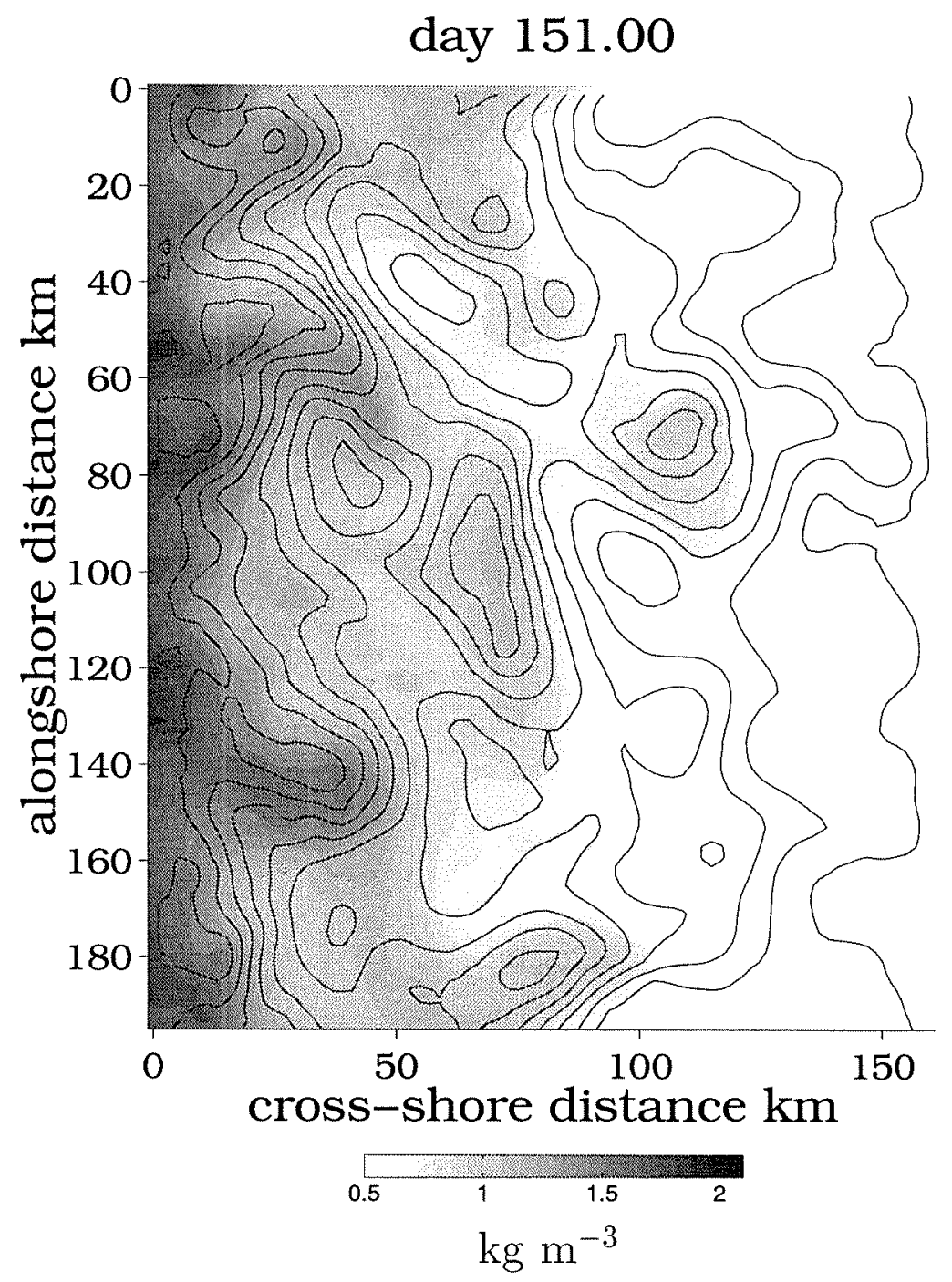

Figure 3.6: The depth averaged density and transport streamfunction for day 151 of the base flat bottom case. The contour interval for the streamfunction is $5 \times 10^{4} \mathrm{~m}^{3} \mathrm{~s}^{-1}$. 

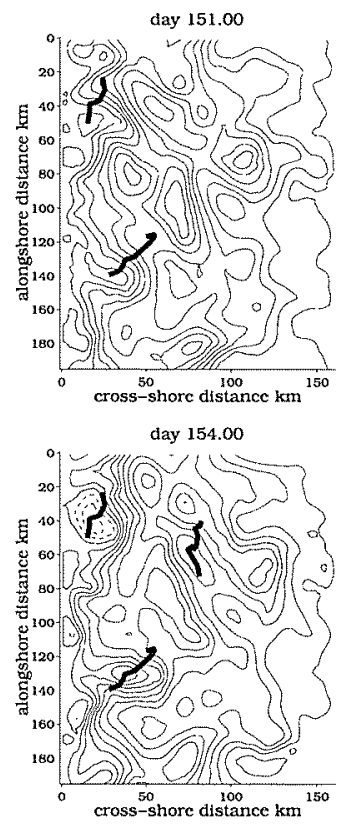

day 157.00

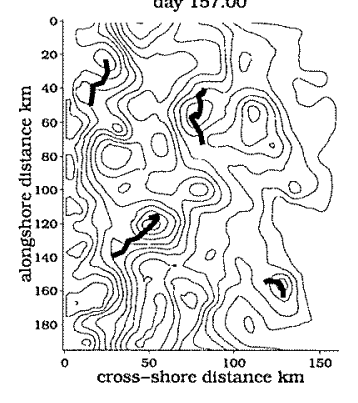

day 160.00

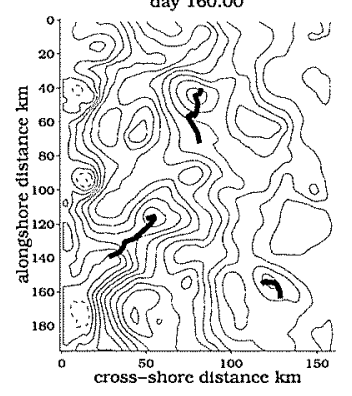

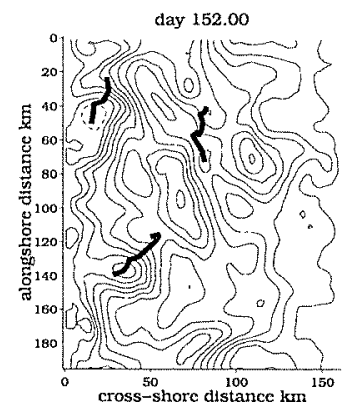

day 155.00

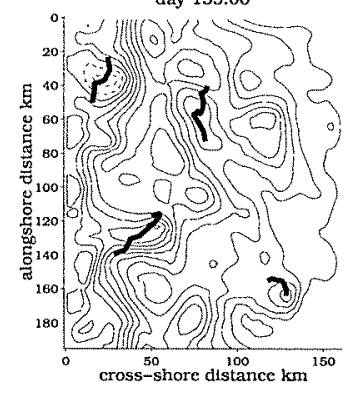

day 158.00

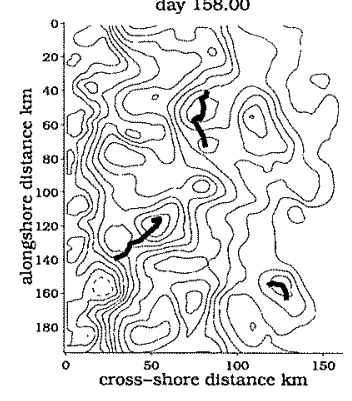

day 161.00

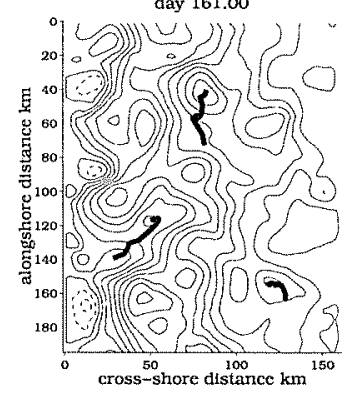

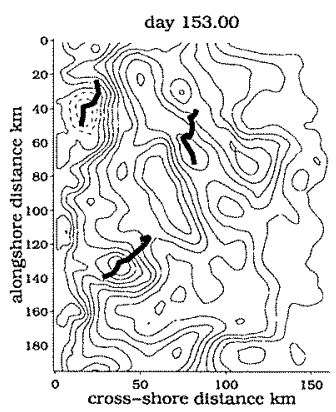

day 156.00

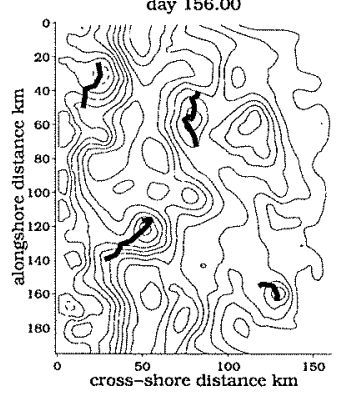

day 159.00

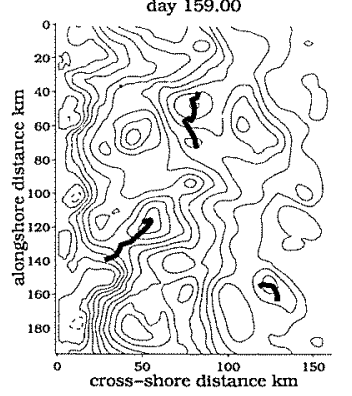

day 162.00

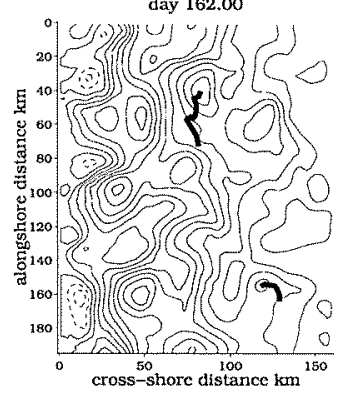

Figure 3.7: The paths of four eddies tracked for 12 days (heavy line) overlaying the transport streamfunction from the base flat bottom case, with bottom friction. The first day corresponds to figure 3.6. The eddy locations are defined by the local maximum or minimum in streamfunction. The paths are only plotted while the eddies exist. The contour interval for the streamfunction is $5 \times 10^{4} \mathrm{~m}^{3} \mathrm{~s}^{-1}$. 


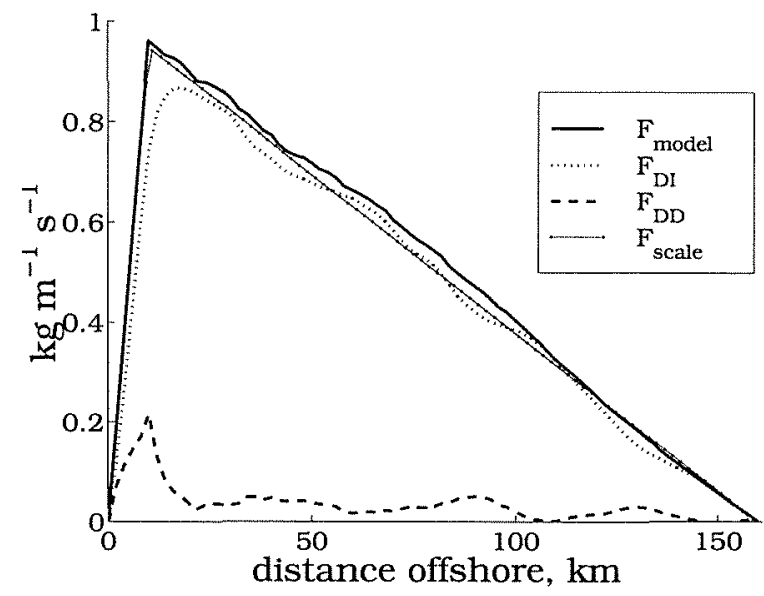

Figure 3.8: The alongshelf averaged depth integrated cross-shelf density flux from the scale (3.9) and the numerical model for the base flat bottom case. The numerical model flux, $F_{\text {model }}$ is broken up into a flux carried by the depth averaged velocity, $F_{D I}$, and flux carried by the depth varying portion of the density and velocity fields, $F_{D D}$. This decomposition is described in section 3.6.1.

computed from the standard deviation and degrees of freedom of $\mathcal{C}$ computed every model day once the model had reached steady state. There is also a systematic error in $\mathcal{C}$ because the domain is periodic in the alongshelf direction, and thus there must be an integer number of eddies in the alongshelf direction. This error is estimated and included in the error bars by calculating what effect a $\pm 0.5 L_{\text {model }}^{2} L_{\text {domain }}^{-1}$ change in the lengthscale $L^{*}$ would have on the cross-shelf density gradient scale, where $L_{\text {model }}$ is the eddy length observed in the model, and $L_{\text {domain }}$ is the alongshelf size of the domain. No estimate of the error induced by cross-shelf quantization was made, since that would be an error in the scaling, not the numerical model. For the fits presented below, $\gamma$, the correlation between the crossshelf depth averaged velocity and the depth averaged density field, is computed from the model runs, and varies between 0.39 and 0.52 . If a fixed $\gamma$ of 0.44 is used, none of the fits change by more than $6 \%$. For checking the assumptions of the scaling, it is most correct to use the $\gamma$ computed in the model. For prediction, one should use the typical $\gamma$ of 


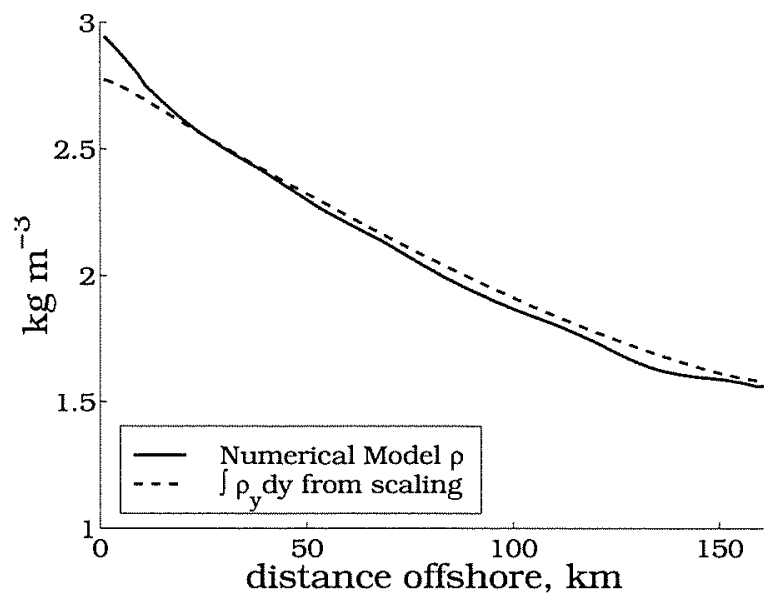

Figure 3.9: The depth and alongshelf averaged density from the base flat bottom model run and from the scaling (3.25). The scale density has not been fit to the density from the full numerical model.

0.44. It is reassuring that this matters little. A compilation of $\gamma$, and other relevant model parameters, is found in table 3.1.

For the base case, the fit $\mathcal{C}$ is $1.05 \pm 0.07$, and the agreement between the depth averaged density in the model and the cross-shelf integral of the scale $\partial \bar{\rho} / \partial y$, shown in figure 3.9 , is good except near the walls at $y=0$ and $y=160 \mathrm{~km}$. Near the walls the scaling goes awry as the length scale of the eddies is inhibited by the vertical walls. It is tempting but incorrect to say this scaling is good because the fit $\mathcal{C}$ is very near one. The scale can be deemed successful if $\mathcal{C}$ is $O(1)$ and if the crossshelf density gradient in the scaling varies as that in the model when the external parameters are varied, i.e. if the fit $\mathcal{C}$ remains a constant as $h, r, Q$, etc. are varied. The scaling (3.23) is thus tested by varying the depth, bottom friction and cooling from the base case, and comparing the percent change in the cross-shelf density gradient predicted by the scaling and observed in the numerical model. The percent 


$\begin{array}{lllll}\text { run number } & \text { geometry } & \gamma & \Delta x & \text { change from base case } \\ \text { f1 } & \text { flat bottom } & 0.45 & 2 & \text { base case } \\ \text { f2 } & \text { flat bottom } & 0.47 & 2 & h / 2 \\ \text { f3 } & \text { flat bottom } & 0.43 & 2 & 2 h \\ \text { f4 } & \text { flat bottom } & 0.48 & 2 & r / 4 \\ \text { f5 } & \text { flat bottom } & 0.44 & 2 & r / 2 \\ \text { f6 } & \text { flat bottom } & 0.39 & 2 & 2 r \\ \text { f7 } & \text { flat bottom } & 0.40 & 2 & Q / 4 \\ \text { f8 } & \text { flat bottom } & 0.44 & 2 & Q / 2 \\ \text { f9 } & \text { flat bottom } & 0.47 & 2 & 2 Q \\ \text { f10 } & \text { flat bottom } & 0.50 & 2 & h / 2, r / 2 \\ \text { f11 } & \text { flat bottom } & 0.51 & 2 & h / 2, r / 4 \\ \text { f12 } & \text { flat bottom } & 0.52 & 2 & h / 2, r / 10 \\ & & & & \\ 1 & \text { local cooling } & 0.31 & 2 & 2 Q, 2 f, 1 / 2 f \\ 2 & \text { local cooling } & 0.38 & 4 & r / 4 \\ 3 & \text { open wedge } & 0.40 & 2 & r / 2 \\ 4 & \text { closed wedge } & 0.43 & 2 & r / 2 \\ 5 & \text { local cooling } & 0.38 & 4 & r / 2 \\ 6 & \text { open wedge } & 0.45 & 2 & 2 f \\ 7 & \text { local cooling } & 0.34 & 4 & \text { double slope } \\ 8 & \text { open wedge } & 0.40 & 2 & \text { base case } \\ 9 & \text { closed wedge } & 0.45 & 2 & 2 Q \\ 10 & \text { closed wedge } & 0.47 & 2 & \text { base case } \\ 11 & \text { local cooling } & 0.38 & 4 & \text { base case } \\ 12 & \text { local cooling } & 0.39 & 2 & \text { slope/1.6 } \\ 13 & \text { local cooling } & 0.42 & 2 & \text { slope/2 } \\ 14 & \text { closed wedge } & 0.47 & 2 & \text { slope/2 } \\ 15 & \text { local cooling } & 0.37 & 4 & 2 r \\ 16 & \text { local cooling } & 0.42 & 2 & \text { slope/5 } \\ 17 & \text { local cooling } & 0.42 & 2 & \text { slope/10 } \\ 18 & \text { local cooling } & 0.45 & 2 & \text { slope/24 } \\ 19 & \text { local cooling } & 0.44 & 2 & \text { slope/60 } \\ 20 & \text { local cooling } & 0.45 & 2 & \text { slope/160 } \\ & & & \end{array}$

Table 3.1: A table of the geometry and changes from the base case for the model runs enumerated in figures 3.11 and 3.10. $\gamma$ is the correlation between the depth averaged $v$ and $\rho$ fields observed in the model. $\Delta x$ is the alongshelf grid spacing in kilometers. $2 Q$ means double the cooling of the base case, $2 f$ means double the rotation rate, etc. $r$ is bottom friction, "Slope" is the bottom slope, $h$ is the water depth. 
change in the model is computed from the fit $\mathcal{C}$ as

$$
\text { \%change }=100\left[\frac{\mathcal{C}}{\mathcal{C}_{\text {base }}} \frac{\partial \bar{\rho}_{\text {scale }}}{\partial y}\left(\frac{\partial \bar{\rho}_{\text {base }}}{\partial y}\right)^{-1}-1\right]
$$

where $\mathcal{C}_{\text {base }}$ is an average of all the $\mathcal{C}$ 's from the $h=100 \mathrm{~m}$ runs. $\mathcal{C}_{\text {base }}=0.98 \pm 0.03$ These comparisons and the corresponding values of $\mathcal{C}$ are given in figure 3.10. The scaling correctly predicts the percent change in the mean density gradient to within the error bars except when the depth is halved to 50 meters. This discrepancy likely occurs because the scalings assume that the Ekman depth is a negligible portion of the water column. In the model runs presented in chapter 2, the Ekman depths were about $10 \mathrm{~m}$, and if all depths are reduced by this amount, the scalings for the $h=50 \mathrm{~m}$ case are improved. The adjusted comparison is also shown in figure 3.10 .

\subsubsection{The Transition Between $\mathcal{L}<1$ to $\mathcal{L}>1$}

$\mathcal{L}$ is the ratio between the Rhines arrest scale $L_{R h}$ and the friction arrest scale $L_{f r}$. Any cascade of energy to large scales will be arrested at the lesser of the two length scales, thus if $\mathcal{L}>1$ the friction length scale should govern the dynamics, and if $\mathcal{L}<1$, the Rhines arrest scale should dominate the dynamics. Thus if $\mathcal{L}<1$, the scale to use is (3.23a) and if $\mathcal{L}>1$, the scale to use is (3.23b). The fit to each scale will be $\mathcal{C}_{R h}$ and $\mathcal{C}_{f r}$ respectively.

In order to test the two scales, many model runs were made over a broad range of $\mathcal{L}$. The models were run with three geometry and cooling configurations. One configuration, called "local cooling," has the same cooling and boundary conditions as the model in the previous section $\left(Q=1 \times 10^{-4} \mathrm{~kg} \mathrm{~m}^{-2} \mathrm{~S}^{-1}\right.$ when $y<10 \mathrm{~km}$, $\left.F_{0}=0\right)$. The bottom is, however, sloping:

$$
h=\alpha\left(y-23.33 \times 10^{3}\right)+100 \text { meters, }
$$


The Boxes are the Predictions of the Scaling of the Percent Change of $\partial \bar{\rho} / \partial y$ From the Base Case, and the Gray Bars are the Numerical Model Results percent change from base case adjusted percent change

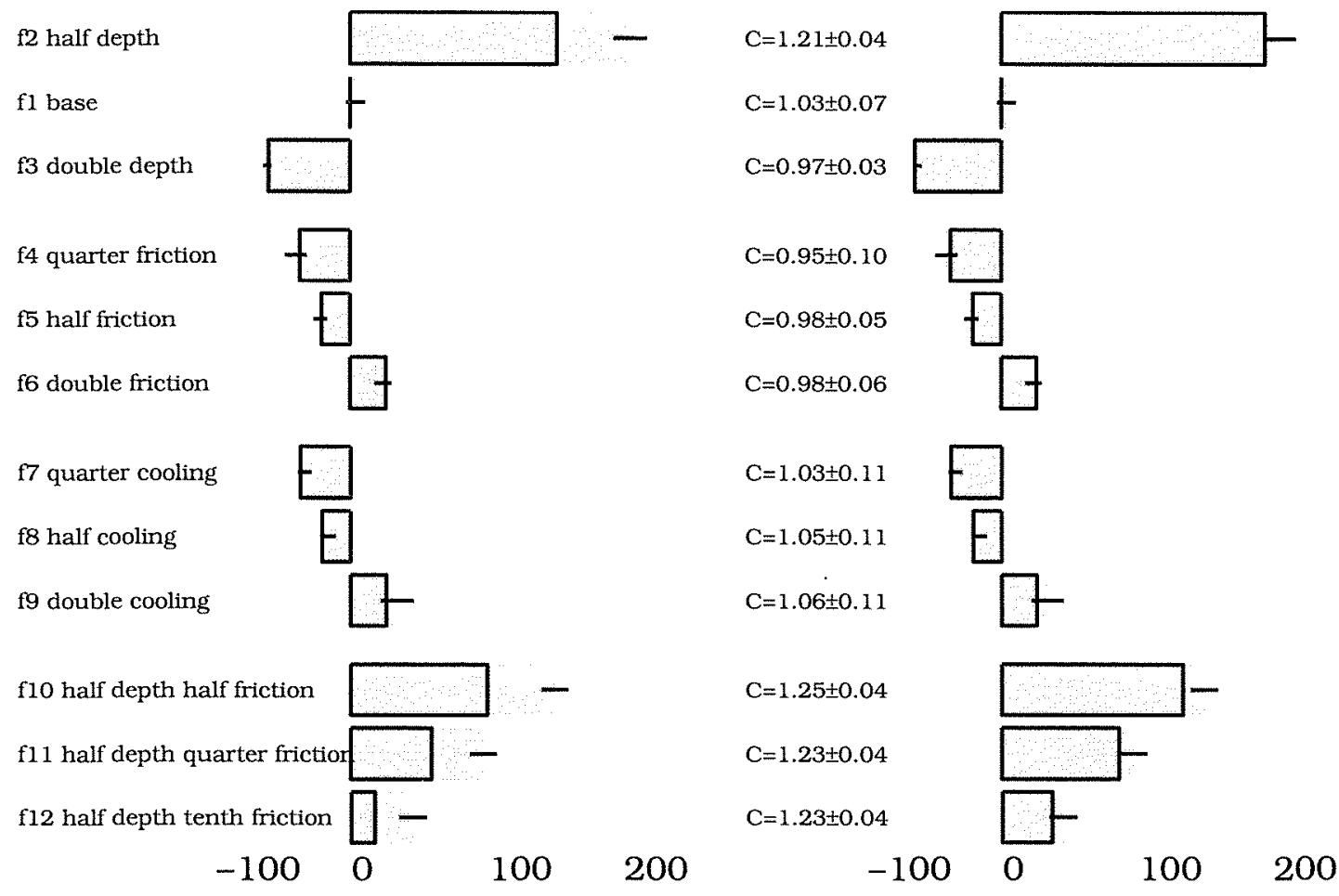

Figure 3.10: The percent change of the cross-shelf density gradient from the base case. The hollow boxes are the predictions from the scaling, the gray filling the results from the numerical model. The horizontal lines are one standard deviation error bars. The righthand side has been adjusted for a finite Ekman depth, the lefthand side has not. 
where $\alpha=0.5 \times 10^{-3}$ in the base case. This geometry and cooling are used for most runs because they are computationally convenient (the water depth does not become very shallow, and $\mathcal{L}$ is nearly constant over the domain). It is also convenient because as $\alpha \rightarrow 0$, the configuration limits to the flat bottom $\mathcal{L}=\infty$ case of the previous section. The two other configurations are more realistic, with a base case density flux of $Q=7 \times 10^{-6} \mathrm{~kg} \mathrm{~m}^{-2} \mathrm{~s}^{-1}$ into the entire domain, equivalent to $300 \mathrm{~W} \mathrm{~m} \mathrm{~m}^{-2}$ out of $3^{\circ} \mathrm{C}$ water or $170 \mathrm{~W} \mathrm{~m}^{-2}$ out of $10^{\circ} \mathrm{C}$ water. In both configurations the bottom slopes uniformly from $10 \mathrm{~m}$ depth, i.e.

$$
h=\alpha y+10 \text { meters, }
$$

where $\alpha=10^{-3}$ in the base case. For one configuration, called the "closed wedge,", $F_{0}=0$, while in the "open wedge" configuration the surface cooling is entirely balanced by the heat flux from the ocean and $F_{0}=Q y_{0}$. For all of the base cases, $r=4.5 \times 10^{-4} \mathrm{~m} \mathrm{~s}^{-1}$, as in the flat bottom base case. For all of the runs, the stated $\mathcal{L}$ will be an average of $\mathcal{L}$ over the region that the fit $\mathcal{C}$ is made.

For each run, $\mathcal{C}_{R h}$ and $\mathcal{C}_{f r}$ are computed. The scalings can be considered successful if $\mathcal{C}_{R h}$ limits to a constant as $\mathcal{L} \rightarrow 0$ and $\mathcal{C}_{f r}$ limits to a constant as $\mathcal{L} \rightarrow \infty$. Physically, this would mean that when $\mathcal{L}<1, \overline{\rho_{y}}$ does not depend on the bottom friction $r$, and when $\mathcal{L}>1, \overline{\rho_{y}}$ does not depend on the background potential vorticity gradient.

In figure $3.11, \mathcal{C}_{R h}$ and $\mathcal{C}_{f r}$ are plotted against $\mathcal{L}$ for a series of model runs whose parameters are given in table 3.1. $\mathcal{C}_{R h}$ and $\mathcal{C}_{f r}$ are computed with $\gamma$ 's computed from the model runs, but, as discussed below, this makes little difference. As $\mathcal{L}$ goes to zero, $\mathcal{C}_{R h}$ goes to 0.65 and $\mathcal{C}_{f r}$ increases. As $\mathcal{L}$ goes to infinity, $\mathcal{C}_{f r}$ goes to 0.98 and $\mathcal{C}_{R h}$ goes to infinity. This is consistent with the scalings derived above, and a 


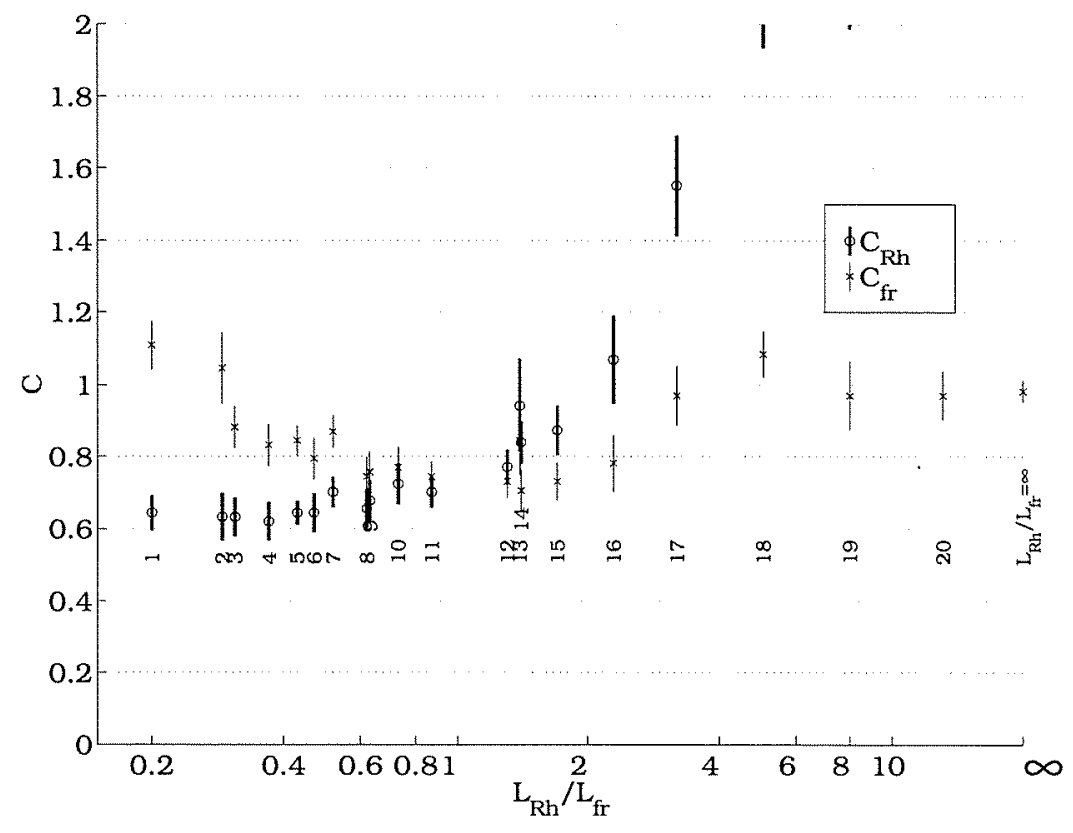

Figure 3.11: The fit between $\bar{\rho}_{\text {scale }}$, and the numerical model for the $\mathcal{L} \leq 1$ case, $\mathcal{C}_{R h}$, and the $\mathcal{L} \geq 1$ case, $\mathcal{C}_{f r}$. $\gamma$ is computed from the model. The fit for $\mathcal{L}=\infty$ is $\mathcal{C}_{\text {base }}$ from section 3.6.1. The error bars are \pm 1 standard error as described in section 3.6.1. 


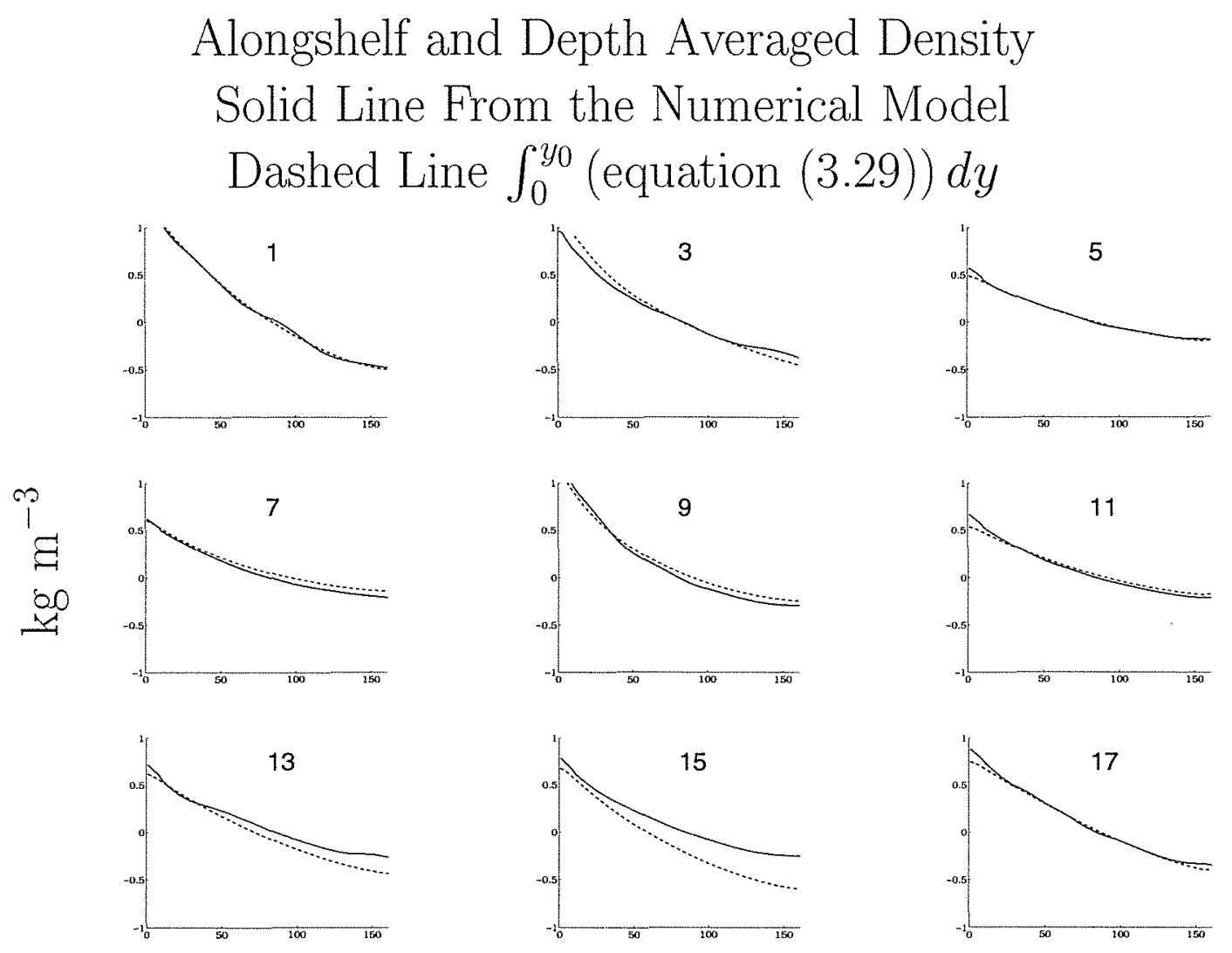

\section{Offshore Distance, km}

Figure 3.12: The alongshore and depth averaged steady state density anomaly from the numerical model runs (solid line) and the cross-shelf integral of (3.29) (dashed line). The run number is on each panel. 
good prediction for $\overline{\rho_{y}}$ can be made with these numbers and (3.23):

$$
\overline{\rho_{y}}=\left\{\begin{array}{ll}
0.98 r^{\frac{1}{3}} \frac{\rho_{0}^{\frac{2}{3}} f^{\frac{2}{3}}}{\gamma^{\frac{1}{3}} h^{\frac{4}{3}} g^{\frac{2}{3}}} F^{\frac{1}{3}} & \text { if } \mathcal{L} \geq 1 \\
0.65 \frac{\partial h^{\frac{1}{5}}}{\partial y} \frac{\rho_{0}^{\frac{3}{5}} f^{\frac{4}{5}}}{2^{\frac{1}{5}} \gamma^{\frac{2}{5}} h^{\frac{6}{5}} g^{\frac{3}{5}}} F^{\frac{2}{5}} & \text { if } \mathcal{L} \leq 1
\end{array} .\right.
$$

$\bar{\rho}_{\text {model }}$ and the cross-shelf integral of (3.29) are shown for every other model run in figure 3.12 .

The two scalings have different implications for coastal oceans. The physics based on the frictional length scale are local, while the Rhines arrest based physics imply the generation of topographic waves which can propagate down the coast. Nonetheless, the shape and character of the eddies does not seem to change in the two limits, as can be seen by comparing figure 3.4 of the $L^{*}=L_{R h}$ limit with figure 3.6 of the $L^{*}=L_{f r}$ limit.

As in the previous section, $\gamma$ is computed from the model runs when the scalings are evaluated. This is appropriate when checking whether the physics of the scaling agrees with the numerical model, but is less useful when trying to make an a priori prediction of $\overline{\rho_{y}}$. Thus it is reassuring that figure 3.11 does not change much when ploted in figure 3.13 with the $\mathcal{C}_{R h}$ and $\mathcal{C}_{f r}$ calculated with a $\gamma$ given by the rule

$$
\gamma= \begin{cases}0.44 & \text { if } \mathcal{L} \geq 1 \\ 0.38 & \text { if } \mathcal{L}<1\end{cases}
$$

Values for $\gamma$ from any model run can be found in table 3.1 .

\subsubsection{Testing the Length and Velocity Scales}

The scales developed for the depth-averaged cross-shelf velocity, $V^{*}$, and the crossshelf length scale, $L^{*}$, are of interest both in their own right and as tools towards understanding sources of error in the theory which underlies (3.23). The Rhines 


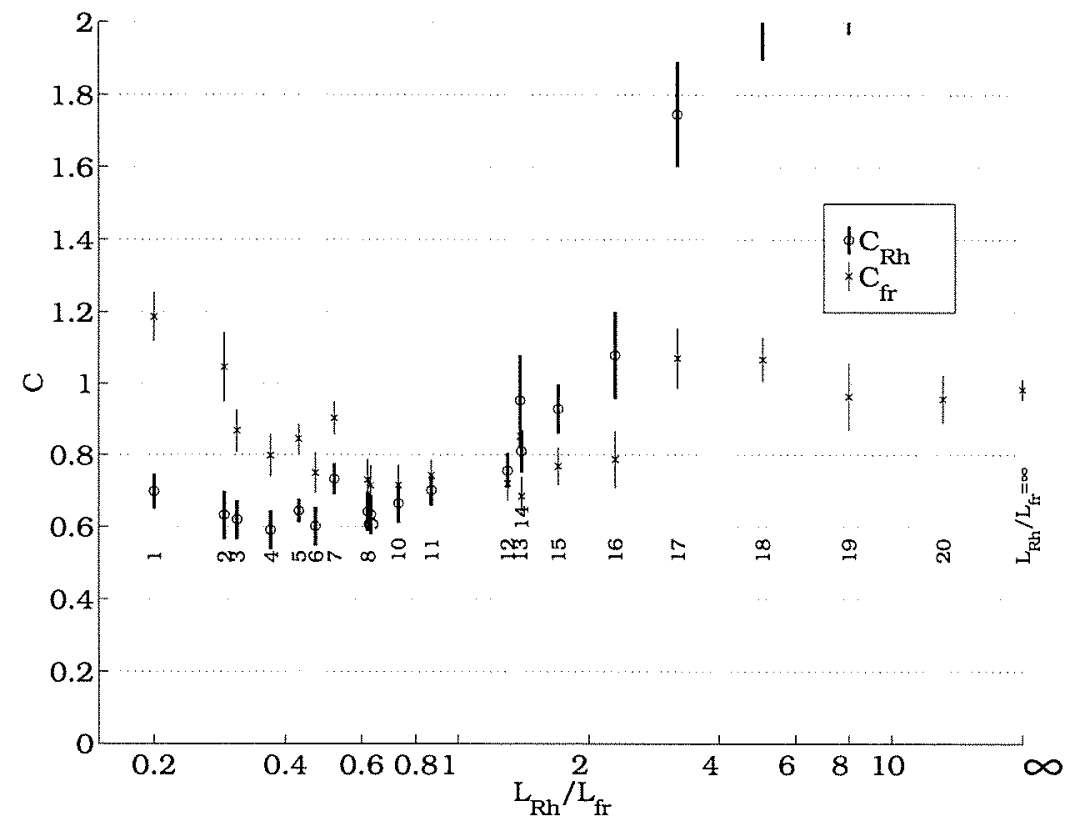

Figure 3.13: The same plot as figure 3.11 except the fits are computed with a $\gamma$ obtained from $(3.30)$. 
arrest scale has been examined in previous work and so this section concentrates on the frictional scalings [LaCasce and Brink, 1998; Rhines, 1977]. The numerical model runs suggest that $V^{*}$ is a good scale for the cross-shelf velocity when $L^{*}$ is $L_{R h}$.

The velocity scalings can be evaluated by calculating $V^{\prime}$

$$
V^{\prime}=\frac{V^{\text {model }}}{V^{*}}=V^{\text {model }} \frac{\rho_{0} f}{g h}\left(\frac{\partial \bar{\rho}}{\partial y}\right)^{-1},
$$

where $V^{\text {model }}$ is $\sqrt{2}$ times the root mean square of the depth averaged cross-shelf velocity, which makes it, hypothetically, the swirl speed of an eddy. It is difficult to calculate the cross shelf length scale of the model since the length scale $L^{*}$ changes in the cross-shelf direction, but the alongshore length scale, $L^{\text {model }}$, can be calculated easily as four times the alongshore decorrelation length scale of the depth averaged density. The alongshore length scale can be related to the cross-shelf length scale with the continuity equation

$$
\frac{U^{\text {model }}}{L^{\text {model }}} \approx \frac{V^{*} V^{\prime}}{L^{\prime} L^{*}},
$$

where $L^{\prime} L^{*}$ is the cross-shelf length scale in in the model and $U^{\text {model }}$ is $\sqrt{2}$ times the root mean square of the depth-averaged alongshelf velocity in the model. The alongshelf length scale and (3.19) can then be used to evaluate $L^{\prime}$

$$
L^{\prime}=\frac{r}{h} \frac{L^{\text {model }}}{U^{\text {model }}} V^{\prime} .
$$

Table 3.2 tabulates the cross-shelf average of $V^{\prime}$ and $L^{\prime}$ for six flat bottom cases where the depth and bottom friction are varied. $\overline{V^{\prime} L^{\prime}}$ is included in the table because the scale $F$ is linearly dependent on it, and $\left(\overline{V^{\prime} L^{\prime}}\right)^{-\frac{1}{3}}$ is included because the frictional scale for $\partial \bar{\rho} / \partial y$ is linearly dependent on it.

If the scalings were completely successful, $h$ and $r$ could be halved or doubled 


$\begin{array}{lcccc}\text { run type } & \overline{L^{\prime}} & \overline{V^{\prime}} & \overline{L^{\prime} V^{\prime}} & \left(\overline{L^{\prime} V^{\prime}}\right)^{-\frac{1}{3}} \\ \text { half depth } & 7.7 & 0.20 & 1.5 & 0.87 \\ \text { base case } & 6.6 & 0.22 & 1.5 & 0.88 \\ \text { double depth } & 5.8 & 0.21 & 1.2 & 0.93 \\ & & & & \\ \text { quarter friction } & 3.9 & 0.49 & 1.9 & 0.81 \\ \text { half friction } & 4.8 & 0.31 & 1.5 & 0.87 \\ \text { base case } & 6.6 & 0.23 & 1.5 & 0.87 \\ \text { double friction } & 9.1 & 0.19 & 1.7 & 0.83\end{array}$

Table 3.2: The cross-shelf average of $L^{\prime}, V^{\prime}, L^{\prime} V^{\prime}$, and $\left(L^{\prime} V^{\prime}\right)^{-\frac{1}{3}}$ for six flat bottom cases.

and $V^{\prime}$ and $L^{\prime}$ would remain constant.

If $h$ were to half and the depth averaged velocities and alongshelf length scales did not change, $V^{\prime}$ would half and $L^{\prime}$ would double. When $h$ is doubled and halved in the model, $V^{\prime}$ remains around 0.22 . When $h$ is increased, $L^{\prime}$ decreases very slowly. The scalings for the velocity and length thus do well as $h$ changes.

If $r$ were to half and the depth averaged velocities and alongshelf length scales did not change, $V^{\prime}$ would not change and $L^{\prime}$ would half. When $r$ is halved in the model, $V^{\prime}$ increases by about $40 \%$ and $L^{\prime}$ decreases by about $30 \%$, and likewise when $r$ is doubled. Thus the scalings over predict the change in the flow field caused by a change in $r$. Indeed,

$$
\frac{r^{\frac{1}{2}}}{h} \frac{L^{\text {model }}}{U^{\text {model }}}
$$

is much more nearly constant then $L^{\prime}$, though it cannot be a complete non-dimensional $L^{\prime}$, for it has units.

These results are consistent with LaCasce [1998], who found that bottom friction inhibited the cascade to the barotropic state (which would reduce $V^{\prime}$ ), but that doubling friction less than halved the decay time of energy (which would increase 
$\left.L^{\prime}\right)$. In a very different calculation, Allen [1984] found that linear coastal-trapped waves on the shelf were damped more slowly than $h r^{-1}$, and that doubling friction did not halve the decay time of the waves. In both Allen's and LaCasce's work, energy loss to the bottom was reduced because the flow in a stratified ocean arranged itself so that the flow near the bottom was weakened.

There is a strong correlation between the inverse of the Burger number computed from the model $\left(L^{\text {model }} / L_{d}\right)$ and $V^{\prime}$, and a corresponding anti-correlation between $L^{\prime}$ and the inverse Burger number in the flat bottom runs (figure 3.14). This is an intriguing result which highlights the need for a scale for the vertical stratification. Similar results were found by Held and Larichev [1995] and Shepherd [1988], even though their work depends on the existence of $\beta$, a background potential vorticity gradient. This is an intriguing result worthy of further work.

Nonetheless, $V^{\prime} L^{\prime}$ remains much more nearly constant than $V^{\prime}$ or $L^{\prime}$ individually

as $h$ and $r$ are varied, and $\left(V^{\prime} L^{\prime}\right)^{-\frac{1}{3}}$ is even more nearly constant. Whether the constancy of $V^{\prime} L^{\prime}$ is fundamental to the dynamics controlling $V^{\prime}$ and $L^{\prime}$ or merely fortuitous, it has the result of making the scale for $\partial \bar{\rho} / \partial y$ more robust than the scales for the velocity and length scale individually.

\subsubsection{Where the Scaling Breaks Down}

The scales presented above breakdown in three limits, when $r$ goes to zero, when $r$ becomes large, and when $f$ becomes small but the Ekman number remains small.

When $\mathcal{L} \ll 1$, alongshore jets form, and in the scales above, it is assumed that energy is removed from the system by the dissipation of these jets by bottom friction. If friction is weak enough, the alongshore jets can become strong enough that the gradients of their potential vorticity are of the same order as $\beta$. At that point, the scalings fail because they assume the relevant potential vorticity gradient is the topographic $\beta$. The physics of flows where the vorticity of jets dominates the mean vorticity has been extensively studied since it is relevant to the dynamics of Jupiter, 

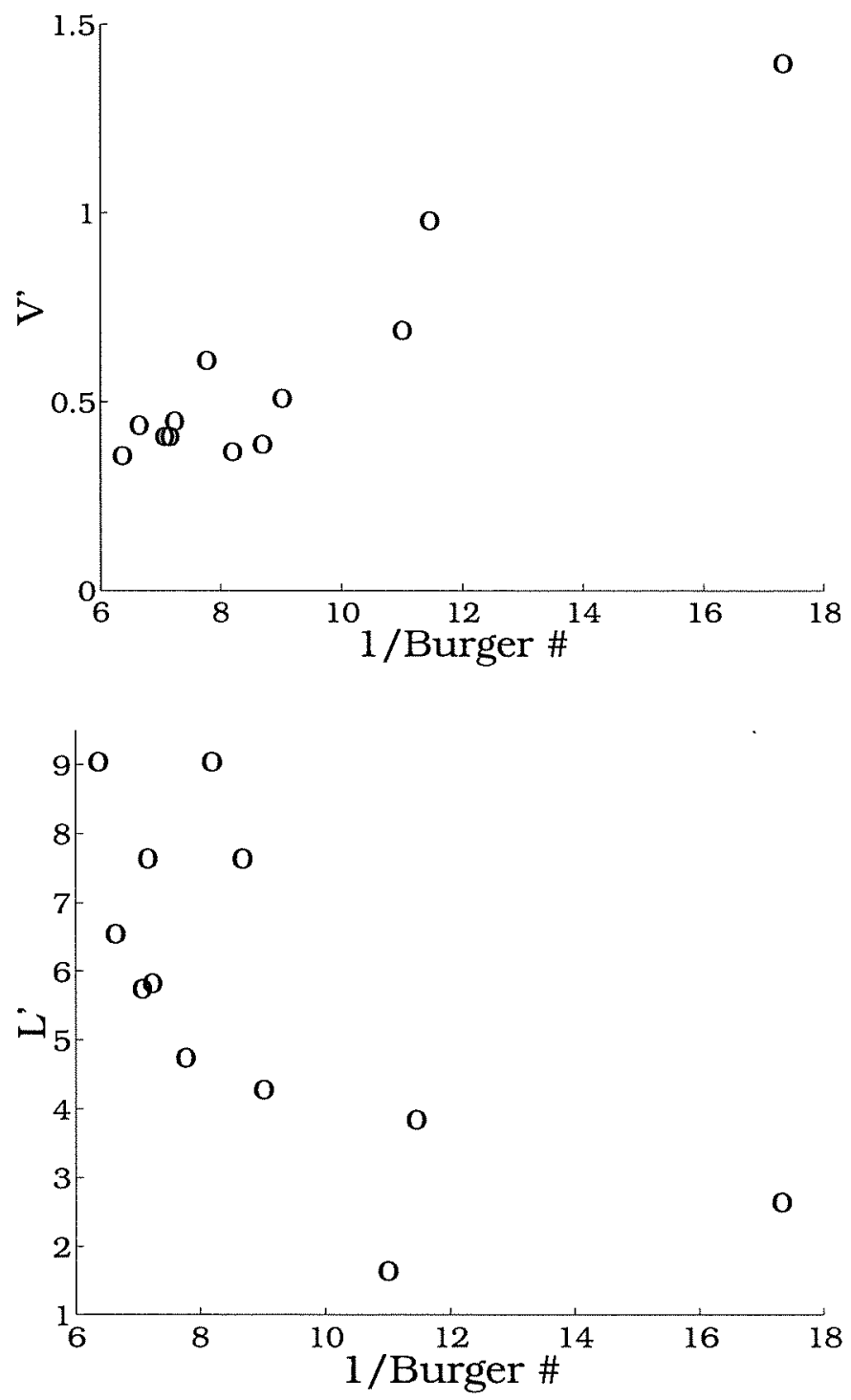

Figure 3.14: $V^{\prime}$ and $L^{\prime}$ against the inverse Burger number $\left(L^{\text {model }} / L_{d}\right)$ as computed from the flat bottom model runs. 
Saturn, and Neptune. A good review of the literature can be found in Dowling [1995]. It is this breakdown in the scalings that prevents the numerical modeling from being extended to $\mathcal{L}<0.15$. ( $\mathcal{L}$ is a relevant parameter because, when $\mathcal{L}<1$, $\mathcal{L}$ is both the ratio of the Rhines arrest scale to the friction length scale and the ratio of the time scale of energy generation, $\mathcal{T}_{\text {advec }}$, to the time scale of energy dissipation, $\left.\mathcal{T}_{\text {fric }}.\right)$ It is unclear if this breakdown of the scalings is relevant in the real ocean because the energy that cascades to the arrest scale can be radiated down the coast, away from the cooling, as coastal trapped waves, a mechanism not available to these model runs which are periodic in the alongshelf direction. For the base case "local cooling" run to breakdown in this fashion, the cooling would have to increase by $\approx 8000$, the friction decrease by 6 , the bottom slope increase by 20 , or the earth's rotation increase by 80 .

Regardless of $\mathcal{L}$, the scalings break down if the length scale predicted by the scaling exceeds the width of the shelf.

When friction increases to the point that the friction decay time is less than an inertial period, $h r^{-1}<2 \pi f^{-1}$, the scalings break down. For the flat bottom runs, as the friction time scale becomes less than the inertial period, more of the cross-shelf flux of density is carried by a mean overturning circulation, and so the fraction of the cross-shelf flux of density carried by the eddies is reduced. Nonetheless, even when the friction time scale is half an inertial period, the error in (3.29) is less than $30 \%$. When there is a bottom slope, the character of the flow changes very quickly when the friction time scale becomes less than an inertial period. When the friction becomes large, the flow breaks up into a series of gravity currents which flow offshore. However, because of rotation, the flow field associated with the gravity currents extend to the surface. This is akin to the gravity current instabilities discussed in Swaters [1991].

The scalings also fail if both vertical mixing and $f$ become small, so that the internal radius of deformation becomes larger than the cross-shelf extent of the 
domain but the Ekman number remains small. This limit leads to a Hadley cell like flow in which a single rotationally affected convection cell occupies the domain. Held and Hou [1980] find the meridional extent of the Hadley cell in the atmosphere to equal an internal radius of deformation as defined by the total meridional (crossshelf) density difference (c.f. James [1994]). Thus the scalings above are likely to be invalid if

$$
\begin{aligned}
y_{0} & <y_{\text {Hadley }}, \\
y_{\text {Hadley }} & =\sqrt{\frac{\Delta \rho g h}{\rho_{0} f^{2}},} \\
\Delta \rho & =\int_{0}^{y_{0}} \frac{\partial \bar{\rho}}{\partial y} d y,
\end{aligned}
$$

where $\partial \bar{\rho} / \partial y$ is from (3.23). $y_{\text {Hadley }}$ is about $10 \mathrm{~km}$ for the runs presented here. Condie and Rhines [1994] analyze a coastal Hadley cell circulation which is appropriate to the $y_{0} \leq y_{\text {Hadley }}$ case.

\subsection{Can the Scalings Above be Used to Under- stand The Transient Behavior of the Density Field?}

The scaling for the cross-shelf flux of density was found under the assumption that the flow was in statistical steady state. However, (3.4) and (3.22) can be written as a diffusion equation

$$
\frac{\partial \bar{\rho}}{\partial t}+\frac{1}{h} \frac{\partial}{\partial y}\left(h A \frac{\partial \bar{\rho}}{\partial y}\right)=\frac{Q}{h}
$$


where

$$
A= \begin{cases}r^{-1} \mathcal{C}_{f r}^{-3} \frac{\gamma g^{2} h^{3}}{\rho_{0}^{2} f^{2}}{\overline{\rho_{y}}}^{2} & \text { if } L=L_{f r}, \text { i.e. } \mathcal{L} \geq 1 \\ 2^{\frac{1}{2}} \mathcal{C}_{R h}^{-\frac{5}{2}} \frac{\partial h^{-\frac{1}{2}}}{\partial y} \frac{\gamma h^{2} g^{\frac{3}{2}}}{\rho_{0}^{\frac{3}{2}} f^{2}} \bar{\rho}_{y}^{\frac{3}{2}} & \text { if } L=L_{R h}, \text { i.e. } \mathcal{L} \leq 1\end{cases}
$$

and it is tempting to use (3.38) to provide a simple model for the time evolution of the depth averaged density field, avoiding the need to resolve the eddies which transport density offshore. However, since the scales in (3.23) assume a spun up eddy-field, this simple model will not be valid until the eddies have had a chance to form and interact. In a field that is everywhere baroclinically unstable, the time scale of the growth of the instabilities is $L_{d} / V^{*}$ (where $L_{d}$ is the internal radius of deformation), and the time scale of the cascade to large scales is $L^{*} / V^{*}$ [Larichev and Held, 1995; Pedlosky, 1987]. $\left(L^{*} / V^{*}=h / r\right.$ if $L^{*}=L_{f r}$, and $L^{*} / V^{*}=\sqrt{2 /\left(\beta V^{*}\right)}$ if $\left.L^{*}=L_{R h}\right)$.

An interesting test of the simple model is given when the cooling is limited to the region within $10 \mathrm{~km}$ of the coast. This problem has been studied by Chapman and Gawarkiewicz [1997] and Spall and Chapman [1998] for a coastal polyna, where the cooling has a finite cross-shore and alongshore extent, but the solution can be extended to include cooling with no alongshore variation. (It is the limit $a \gg b$ in Chapman and Gawarkiewicz [1997] with $W=L_{d}$ [Chapman, 1998]). In numerical model runs without bottom friction but with a flat bottom, Spall and Chapman [1998] and Chapman [1998] find that the density averaged over the cooling region stops increasing in a time

$$
t_{\text {steady }} \approx\left(\frac{2}{c_{e}}\right)^{\frac{2}{3}}\left(\frac{\rho_{0} y_{\text {cool }}^{2}}{g Q}\right)^{\frac{1}{3}}
$$


and the steady state density is

$$
\rho_{\text {steady }} \approx \frac{1}{h}\left(\frac{2}{c_{e}}\right)^{\frac{2}{3}}\left(\frac{\rho_{0} y_{\text {cool }}^{2}}{g}\right)^{\frac{1}{3}} Q^{\frac{2}{3}}
$$

where the scalings have been written for the cooling geometry and notation used here. The length $y_{\text {cool }}$ is the offshore extent of the cooling. Spall and Chapman [1998] interpret $c_{e}$ as the ratio of the propagation speed of hetons moving away from the cooling region and the swirl speed of the eddies which compose the heton. The swirl velocity is scaled with thermal wind. In Spall and Chapman [1998], as illustrated in the top panel of the cartoon in figure 3.15, these hetons are assumed to carry dense water away from the cooling region, and the cooling region is assumed to spawn hetons frequently enough to keep the density in steady state. They find, from theory, that $c_{e}$ is about 0.04 , so the hetons leave the cooling region at a speed about $1 / 25$ of their swirl speed.

In figure 3.16, the evolution of the mean density of the cooling region is plotted for two full numerical model runs, each with the same parameters as the base case of section 3.6 .1 (cooling inshore of $10 \mathrm{~km}, Q=10^{-4} \mathrm{~kg} \mathrm{~m}^{-2} \mathrm{~s}^{-1}, h=100 \mathrm{~m}$ ), but one with bottom friction $\left(r=4.5 \times 10^{-4} \mathrm{~m} \mathrm{~s}^{-1}\right)$ and one without $(r=0)$.

In the full numerical model run without bottom friction, the density averaged over the cooling region is seen to reach a nearly steady state of about $0.4 \mathrm{~kg} \mathrm{~m}^{-3}$ at day 8 , which agrees well with the prediction of about $0.6 \mathrm{~kg} \mathrm{~m}^{-3}$ from (3.41). (Spall and Chapman [1998] discuss why using the theoretical $c_{e}$ will slightly underestimate the steady state density.) There is a very small upward trend in the density between days 8 and 40, perhaps because the water entering the cooling region to replace the water removed by the hetons has been slightly cooled by the previous hetons leaving the domain. This upward trend is so slight that it is not visible if the density is averaged over an alongshore extent which contains only four or five eddies. The density begins to increase more rapidly after day 40 when the eddies are stopped by 


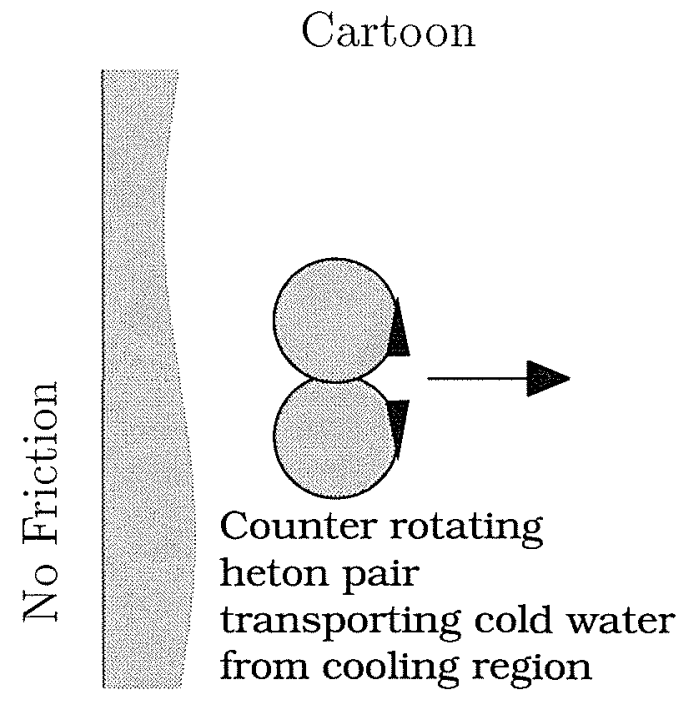

Numerical Model $\Psi$ and $\bar{\rho}$
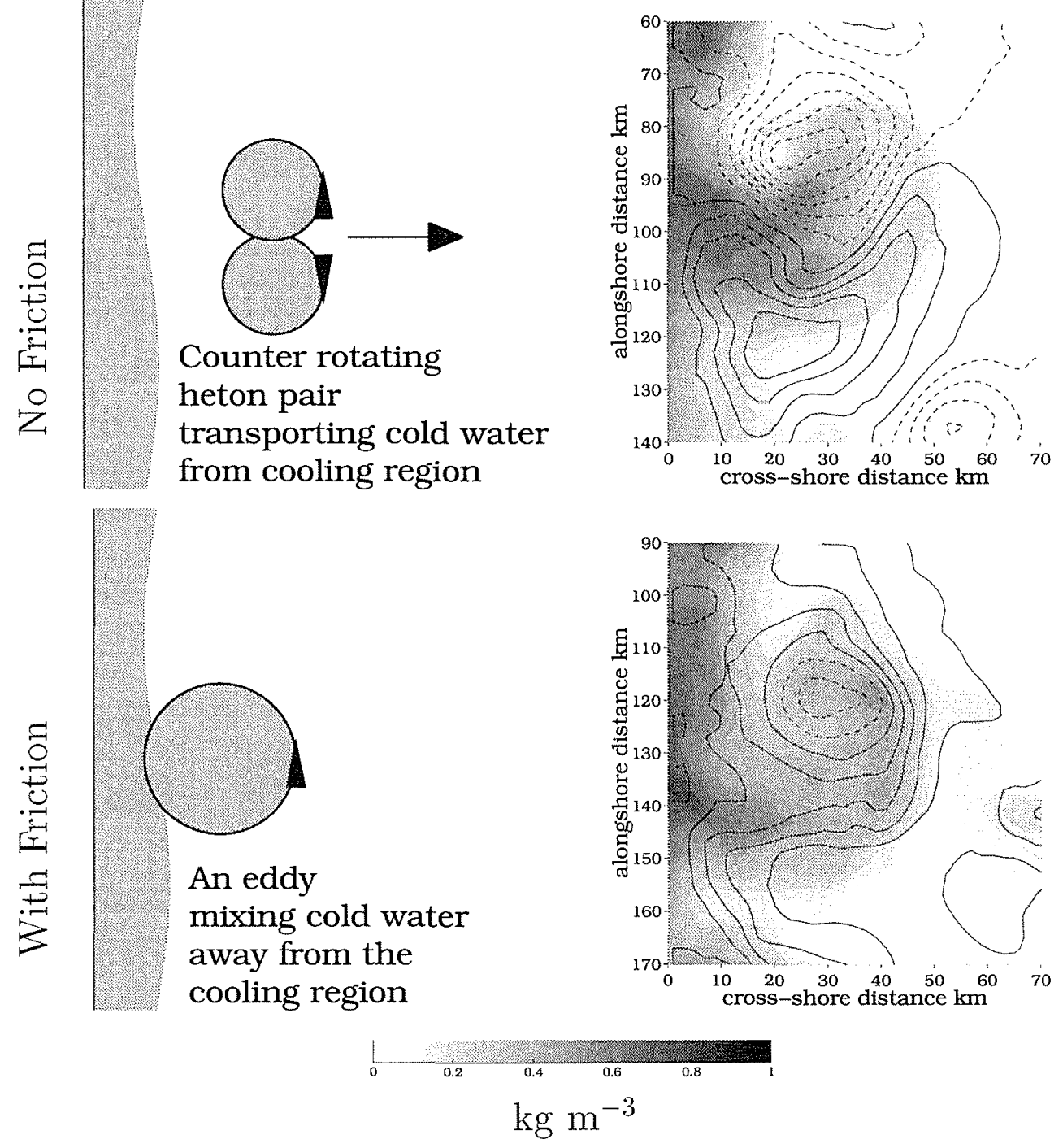

Figure 3.15: Two cartoons illustrating eddy transport of heat offshore in a model with and without bottom friction, and plots of the barotropic streamfunction overlaying the depth averaged density from day 20 of two corresponding model runs. Negative streamlines are dashed, and the contour interval is $5 \times 10^{4} \mathrm{~m}^{3} \mathrm{~s}^{-1}$. 


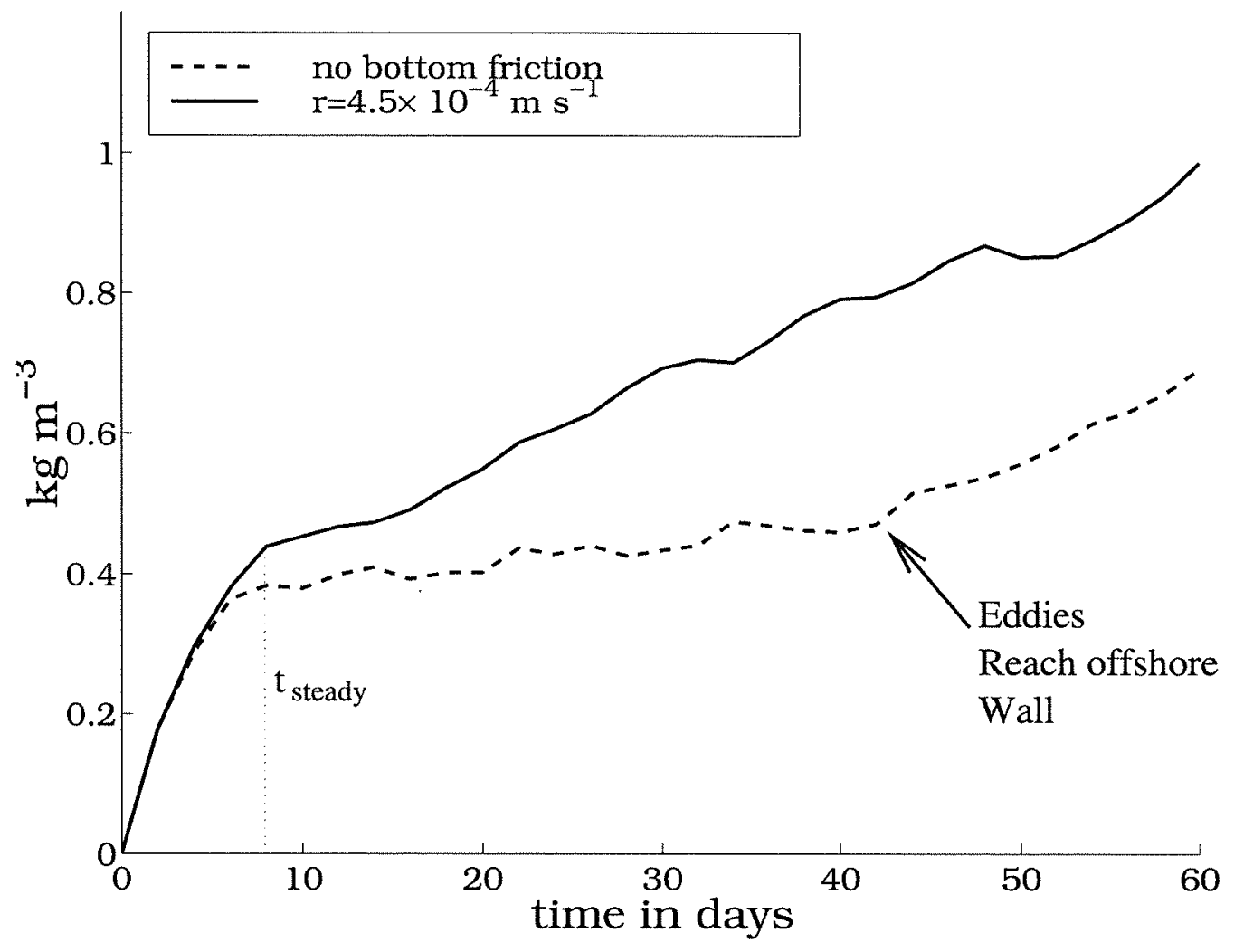

Figure 3.16: The average density of the cooling region for two full numerical model runs with cooling limited to within $10 \mathrm{~km}$ of the coast. Each curve is the result of the average of four model runs, each initialized with an RMS noise in the density field of $10^{-3} \mathrm{~kg} \mathrm{~m}^{-3}$.

the offshore wall and prevented from removing heat from the domain. Because the offshore wall is, to some extent, artificial, Chapman and Gawarkiewicz [1997] stop their model runs before eddies reach it.

In the full numerical model run with bottom friction, however, the density in the cooling region does not reach a steady state but always increases as only part of the surface cooling is balanced by a cross-shelf eddy driven heat flux. Understanding the difference between these two cases is important to understanding when the simple model (3.38) becomes valid.

What prevents the steady state in the frictional case? The model of heton 
propagation in Hogg and Stommel [1985] and Spall and Chapman [1998] has only been formalized in layered systems, where the heton is composed of two horizontallyoffset counter-rotating eddies, one intensified at the surface, one at the bottom. Either of these two eddies alone would not propagate horizontally, but the pair together can. Bottom friction in the layer model would consume the bottom eddy, leaving the surface intensified eddy unable to propagate across the shelf. The surface intensified eddy would then be trapped, unable to escape its generation region at the boundary of the cooling region. This is illustrated in the lower panel of figure 3.15. (A similar mechanism for disrupting heton propagation over a frictionless sloping bottom is given in LaCasce [1996].)

It is reasonable to expect similar dynamics in continuously stratified flows, and thus to expect friction to retard the cross-shelf propagation of hetons within a friction time scale $(h / r)$ of their generation. Once bottom friction prevents the eddies from propagating across the shelf, the heton mechanism posited by Spall and Chapman [1998] for maintaining a steady state density in the cooling region breaks down, and there is no reason to expect the density in the cooling region to remain steady. Once the eddies are no longer able to propagate across the shelf, they will begin to interact with other eddies created at the boundary of the cooling region. Since the friction time scale is about 2.5 days in the base flat bottom case, short compared to the time scale for the achievement of a steady state density in the cooling region in the inviscid case, (3.40), it is unlikely that heton dynamics are ever important in the run with bottom friction. Figure 3.17 shows the streamfunction and the sense of the relative vorticity for day 8 both with and without bottom friction, and the difference is striking: the case without bottom friction is populated by counter-rotating pairs of eddies, while the case with bottom friction contains almost exclusively cyclonic eddies. The positive relative vorticity anomalies are also strengthened relative to the negative anomalies in the frictional case. There is scant evidence of heton pairs in the case with bottom friction. The cross-shelf structure of the density fields in 


\section{Transport Streamfunction $\Psi$ \\ Shaded where $\nabla^{2} \Psi>0$}

\section{No Friction}

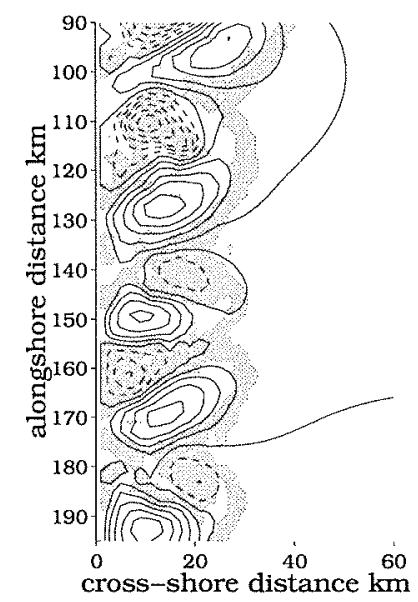

Friction

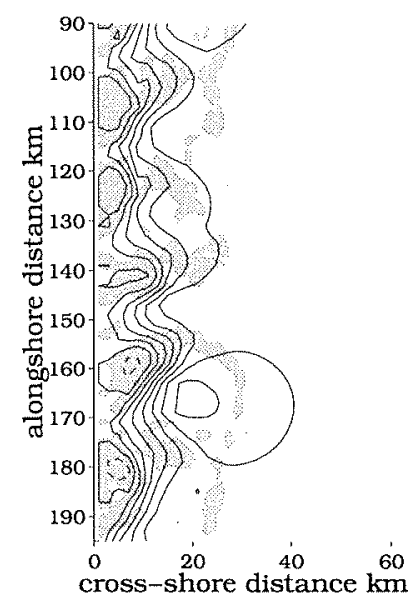

Figure 3.17: The transport streamfunction $\Psi$, dashed where negative and shaded where $\nabla^{2} \Psi>0$. Day 8 of base case geometry and forcing, with and without bottom friction. Note the lack of counter rotating eddy pairs in the run without bottom friction.

the base frictional case and the no bottom friction case are compared in figure 3.18, and the faster transport of density across the shelf in the latter case can be seen. Once the eddies begin to interact, it becomes impossible to track any one eddy a significant distance across the shelf, for new eddies are continuously being spawned only to be sheared apart a few days later (c.f. figure 3.7).

Once the eddies in the model have grown vigorous enough to make the heat balance in the cooling region no longer one dimensional (which in all cases described below occurs in a time predicted well by the scalings in Chapman and Gawarkiewicz [1997] given in (3.40)), and a time comparable to the cascade to larger scales has passed, the simple model of equation (3.38) describes the evolution of the depth averaged density. Equation (3.38) can be integrated numerically with the same boundary conditions as on (3.4). The solution for the base friction case are compared to $\bar{\rho}$ from the model in figure 3.19, and the solution to the simple model agrees well with the full numerical model after day 14. The density near the coast is slightly 
No Friction
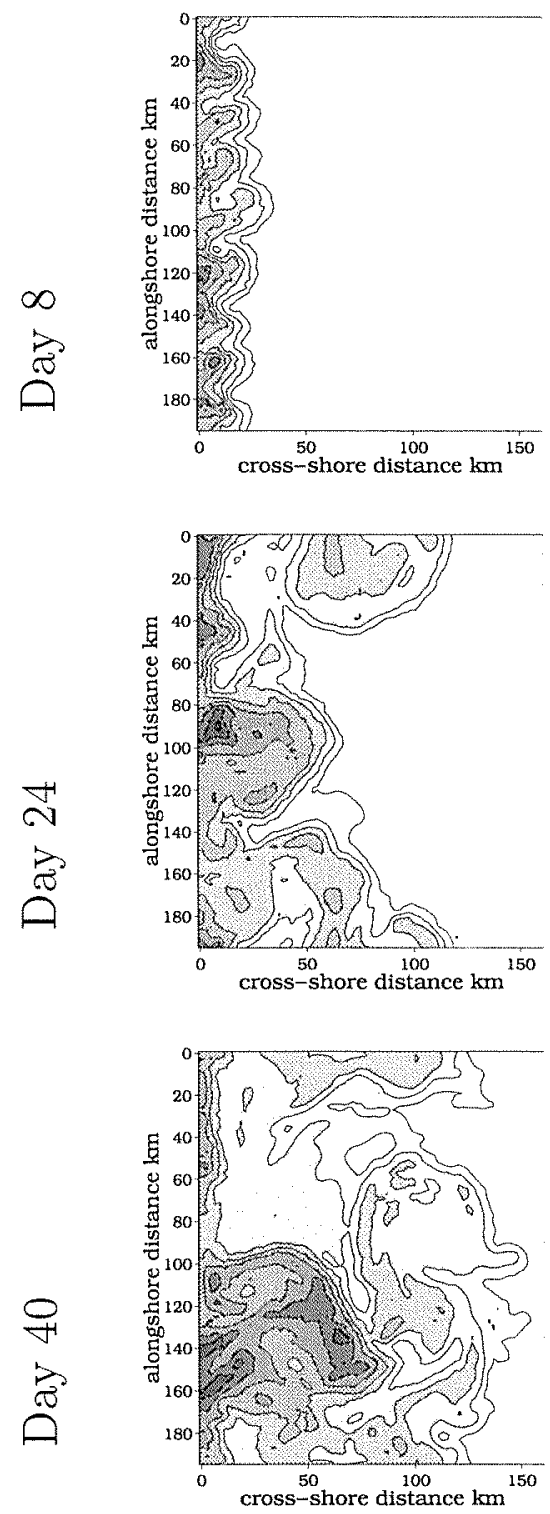

Friction
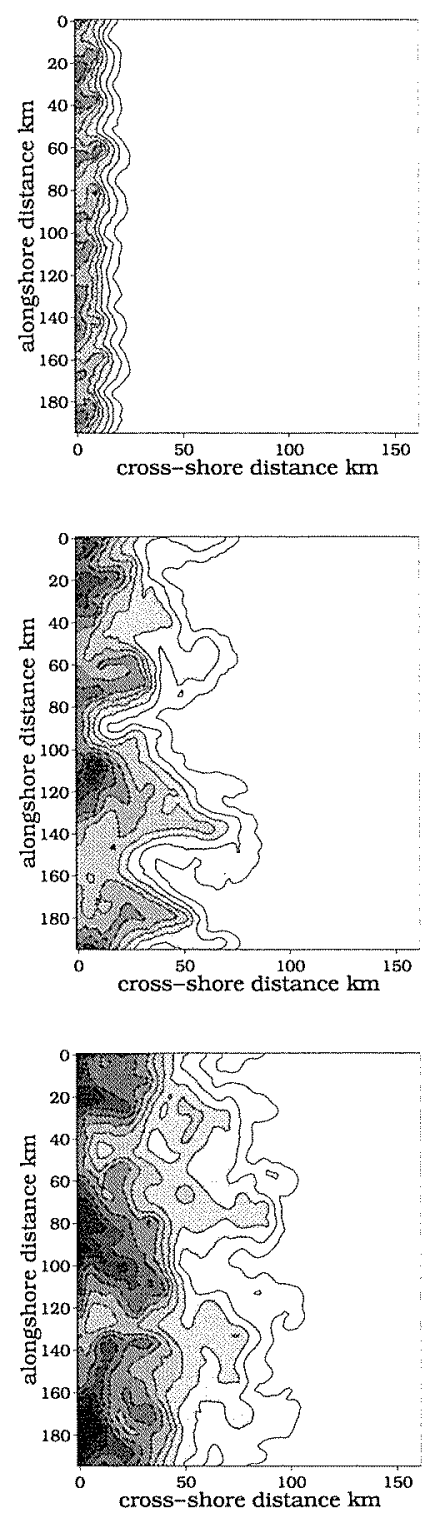

$\mathrm{kg} \mathrm{m}^{-3}$

Figure 3.18: The depth averaged density for the base flat bottom case, and for a model with the same geometry and forcing but no bottom friction. Plotted for days 8, 10, and 40 after the inception of cooling. 
Frictional case, $r=4.5 \times 10^{-4} \mathrm{~m} \mathrm{~s}^{-1}$

Dashed Line is $\bar{\rho}$ from Simple Model

Solid Line is $\bar{\rho}$ from Primitive Equation Model
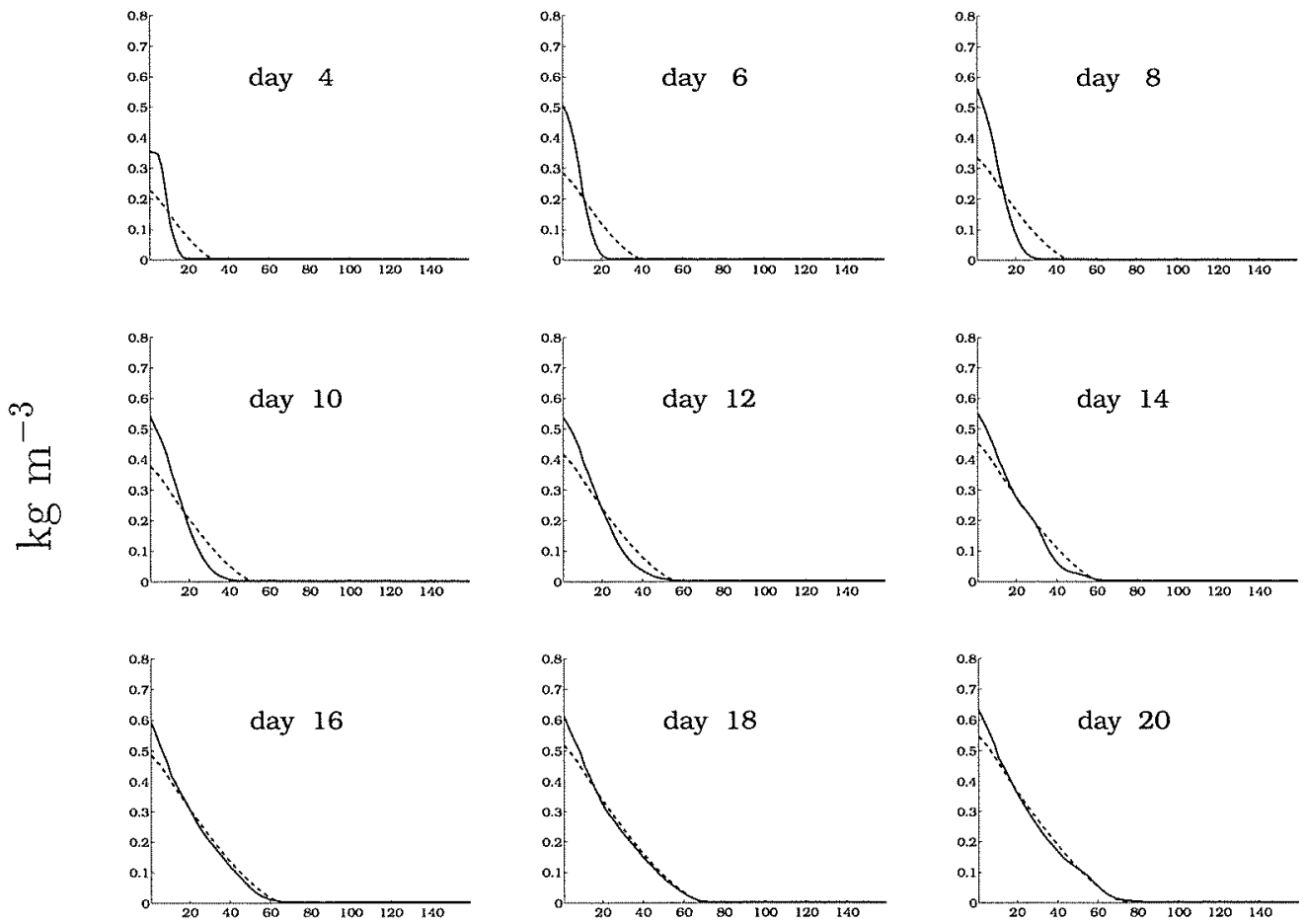

\section{$\mathrm{km}$ offshore}

Figure 3.19: The solid line is $\bar{\rho}$ from the full numerical model run of the base flat bottom case, while the dashed line is $\bar{\rho}$ from the simple model. As before, four full numerical model runs were averaged to find $\bar{\rho}$.

high because the length scale of the eddies are reduced near the coastal wall by the wall. $\left(\mathcal{C}_{f r}\right.$ for the simple model presented here and later is taken from (3.29) and $\gamma$ from (3.30).)

The density averaged over the cooling region is computed from both the simple model and the full numerical model for the base case and for runs with friction one half, one quarter and one eighth the base friction. These results are compared with the no bottom friction case in figure 3.20. In none of the cases with bottom friction is the density in the cooling region constant, and in all cases the simple 
model accurately describes the density increase of the cooled region after the initial transient.

The simple model cannot describe the behavior of the run with no bottom friction, for $L_{f r}$ and $L_{R h}$ are both infinite, causing the simple model to break down (c.f. 3.6.4). The model of Chapman and Gawarkiewicz [1997] breaks down when the eddies encounter the offshore wall on day 40. While perhaps of little relevance to an actual ocean, it is interesting to note that once the eddies are confined by the offshore wall, they merge to fill the entire domain with a single eddy, as predicted by models of $f$-plane turbulence [Larichev and Held, 1995]. A snapshot of this domain-filling eddy is given in figure 3.21 .

$L^{*}=L_{f r}$ for all of the flat bottom runs, and so to test the simple model when $L^{*}=L_{R h}$, a run was made with half the cooling of the base flat bottom case $\left(5 \times 10^{-5}\right.$ $\mathrm{kg} \mathrm{m} \mathrm{m}^{-2} \mathrm{~s}^{-1}$ inshore of $10 \mathrm{~km}$ ), with a depth of 50 meters at the coast and increasing with a slope of $10^{-3}$ offshore, and with a bottom friction of $5 \times 10^{-4} \mathrm{~m} \mathrm{~s}^{-1} \cdot \mathcal{L}=0.5$ when the model is fully spun-up. The geometry, forcing, and bottom friction are the same as that used in figures 10 and 11 of Chapman and Gawarkiewicz [1997], except for the infinite alongshore extent of the cooling. This is the only model run they present which includes bottom friction or bottom slope. A plot of the evolution of the average density of the cooling region in the full numerical model and the simple model is given in figure 3.22. The increase in the average density of the cooling region slows between day six and eight in the full numerical model, which agrees well with the estimate of Chapman and Gawarkiewicz [1997] of 6.3 days (3.40). The density remains nearly steady until day 20, when it begins to agree with the simple model and increase. The apparent steady state density of the cooling region shown in figure 10 of Chapman and Gawarkiewicz [1997] is likely an illusory one caused by their stopping of the model run at day 24. They are forced to stop the model run at day 24 when the eddies begin to reach their "offshore" boundary, $75 \mathrm{~km}$ from the coast. In the model run shown in figure 3.22 , the offshore boundary is $160 \mathrm{~km}$ 


\section{Density Under Region Being Cooled}

Solid Line is Primitive Equation Model

Dashed Line is Simple Model
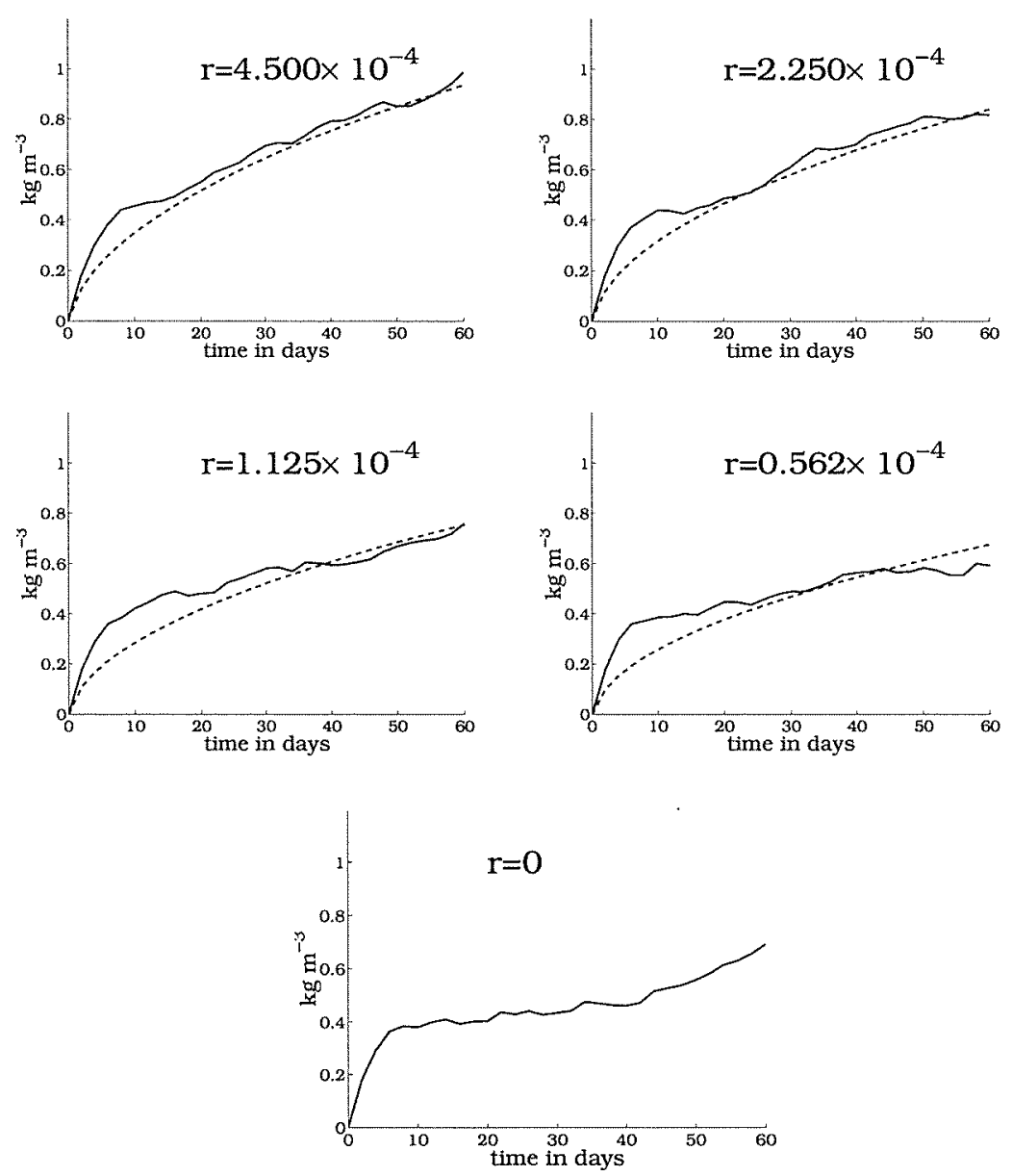

Figure 3.20: The average density beneath the cooling region for four flat bottom runs made with varying bottom friction, and one with no bottom friction. The geometry and cooling are as the base flat bottom case. The solid line is from the full numerical model, and the dashed line is from the simple model. As before, four full numerical model runs were averaged to find the density beneath the cooling region. The units of $r$ are meters per second. No simple model run is made for the run without bottom friction because the simple model is not valid when there is no bottom friction. 
Depth averaged $\rho$ day 140

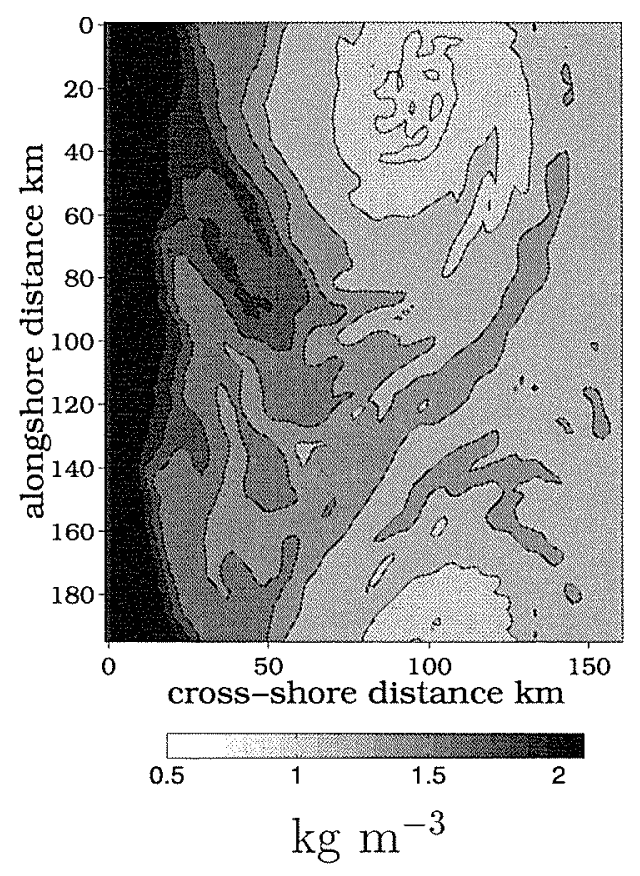

Figure 3.21: The depth averaged density from day 140 of a model run whose geometry and forcing were the same as the base flat bottom case, but with no friction. 
offshore, and the eddies do not reach it until day 50, well after the density of the cooling region began to increase again at day 20.

It is possible, however, that the steady state shown in figure 10 of Chapman and Gawarkiewicz [1997] would persist if the model domain were enlarged and the model run for a longer time, because in their model the cooling has a limited alongshore extent. The eddies could avoid interacting by propagating along the shelf (though their figures do not seem to show much alongshelf eddy propagation at the time their model run is stopped.) This illustrates a weakness of the simple model given by (3.38): since it is based on forcing which does not vary in the alongshelf, it cannot, yet, be used for forcing that varies in the alongshelf direction.

Even though the simple model does well in the runs above, it still could not be used to model completely a coastal ocean undergoing winter time cooling. Even if the forcing is assumed not to vary in the alongshore, the simple model is not complete. Some failures of the simple model can be illustrated by several simple thought experiments. If the ocean being modeled has a cross-shelf density gradient, sloping bottom, but no cooling:

$$
\begin{aligned}
h & =\alpha y+h_{0}, \\
Q & =0 \\
\frac{\partial \rho}{\partial y} & =\text { a constant }
\end{aligned}
$$

and if the slopes of the isopyncnals are less than the slope of the bottom:

$$
\frac{\partial \rho}{\partial y}\left(\frac{\partial \rho}{\partial z}\right)^{-1}<\alpha
$$

the flow is stable to infinitesimal perturbations, and thus there will be no instabilities to transport heat across the shelf [Blumsack and Gierasch, 1972; Pedlosky, 1987]. Nevertheless, the simple model would predict a cross-shelf eddy driven density flux and thus an evolving density field simply because there is a mean cross-shelf density 


\section{Density Under Region Being Cooled}

Solid Line is Primitive Equation Model

Dashed Line is Simple Model

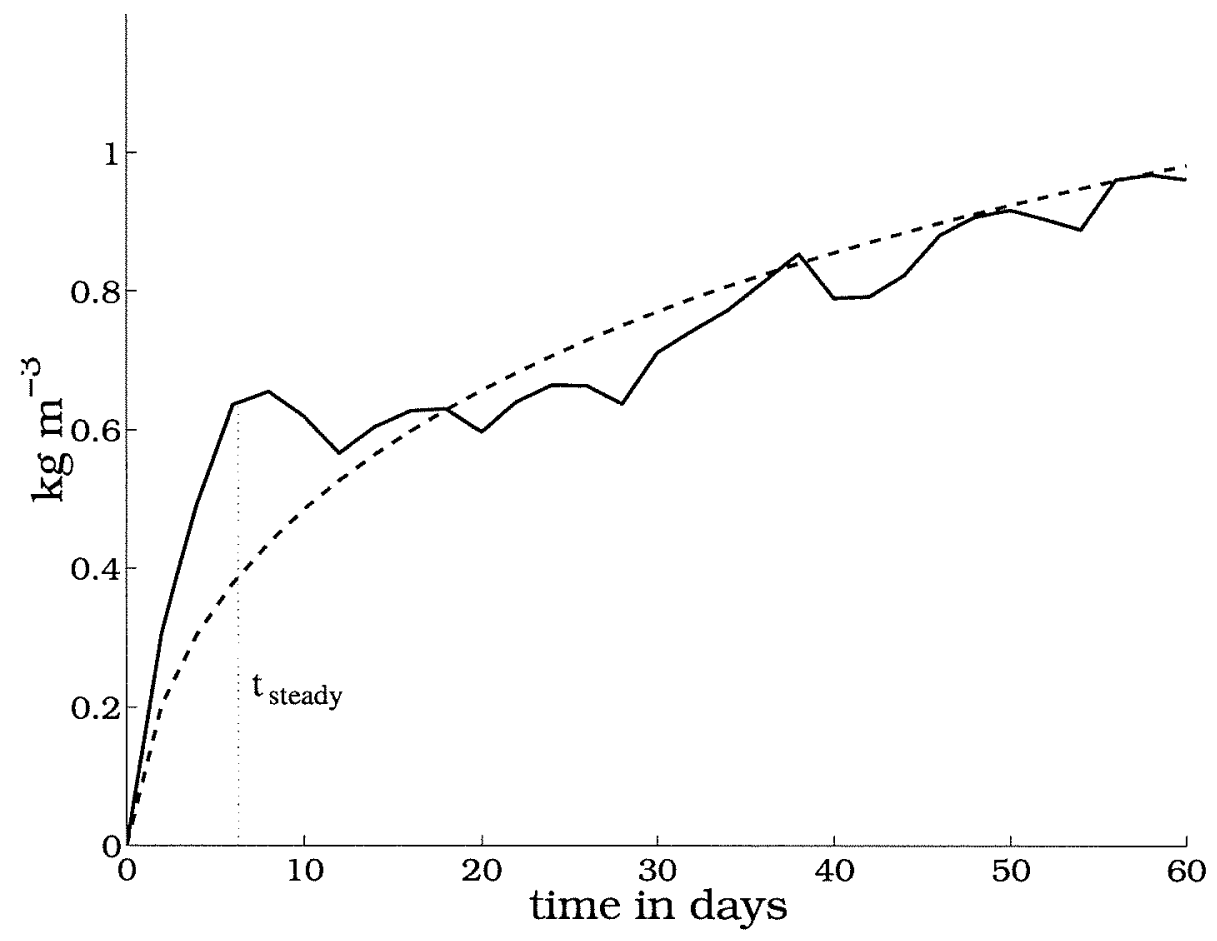

Figure 3.22: The average density beneath the cooling region for a run made with half the cooling of the base flat bottom case $\left(5 \times 10^{-5} \mathrm{~kg} \mathrm{~m}^{-2} \mathrm{~s}^{-1}\right.$ inshore of $\left.10 \mathrm{~km}\right)$, with a depth of 50 meters at the coast and increasing with a slope of $10^{-3}$ offshore, and with a bottom friction of $5 \times 10^{-4} \mathrm{~m} \mathrm{~s}^{-1}$. The solid line is from the full numerical model, and the dashed line is from the simple model. As before, four full numerical model runs were averaged to find the density beneath the cooling region. 
gradient. (This case cannot happen forever when there is cooling, for when there is cooling and no cross-shelf flux, $\partial \bar{\rho} / \partial y$ increases until the the system is unstable.)

Another case where the simple model breaks down is described in section 3.2 , where the vertical mixing caused by cooling extends to the bottom and the entire water column is statically unstable. As described in chapter 2, when this occurs on the shelf, geostrophic motions are inhibited by vertical mixing and the heat balance is one dimensional. Nevertheless, the simple model would predict a cross-shelf heat flux regardless of the mixing as long there was a cross-shelf density gradient.

It would be difficult to modify the simple model to account for these cases, because the simple model does not predict the vertical stratification needed to diagnose whether convection extends to the bottom, and whether the flow field is baroclinically unstable. Thus while the simple model provides insight into the time evolution of the density field when the cross-shelf density flux is dominated by eddies in the flow field, it is not a general purpose parameterization for the flux of density across the shelf.

In order to extend the simple model, one could start by finding a scale for the vertical stratification, and then devising tests for the scenarios above. The question of how the turbulent field reacts to wind driven flows also needs to be addressed for a more complete simple model.

\subsection{Extending the Scalings to Passive Tracers}

In the previous sections, the depth-integrated cross-shelf density flux was modeled successfully as

$$
F=h \gamma V^{*} L^{*} \overline{\rho_{y}}
$$


and it seems reasonable to attempt to model the transport of a passive tracer $c$ in the same fashion:

$$
\begin{gathered}
F_{c}=h \gamma V^{*} L^{*} \overline{c_{y}} \\
\frac{\partial \bar{c}}{\partial t}+h^{-1} \frac{\partial F_{c}}{\partial y}=0 .
\end{gathered}
$$

Again modeling the transport as a diffusive process and assuming the flow has reached a steady state, (3.45), (3.20), and (3.12) can be written:

$$
\begin{gathered}
A=\gamma V^{*} L^{*}= \begin{cases}\mathcal{C}_{f r}^{-1} \frac{\gamma^{\frac{1}{3}} g^{\frac{2}{3}} h^{\frac{1}{3}}}{r^{\frac{1}{3}} \rho_{0}^{\frac{2}{3}} f^{\frac{2}{3}}} F^{\frac{2}{3}} & \text { if } \mathcal{L} \geq 1 \\
2^{\frac{1}{5}} \mathcal{C}_{R h}^{-1} \frac{\gamma^{\frac{2}{5}} g^{\frac{3}{5}} h^{\frac{1}{5}}}{\frac{\partial h}{\partial y} \rho_{0}^{\frac{1}{3}} f^{\frac{4}{5}}} F^{\frac{3}{5}} & \text { if } \mathcal{L} \leq 1\end{cases} \\
\frac{\partial \bar{c}}{\partial t}+h^{-1} \frac{\partial}{\partial y} h A \frac{\partial \bar{c}}{\partial y}=0
\end{gathered}
$$

where $\mathcal{C}$ is the fit found in the previous sections. The value of $\gamma$ is assumed to be the same for both density and passive tracer, which is an assumption that the arrest or friction scale is much larger than the radius of deformation [Rhines, 1977].

However, when the time and space scales of the tracer are small compared to the eddy scales, diffusion becomes an inappropriate model for the turbulent mixing process. The reason for this is explained in detail in Davis [1987], and is explained heuristically below: the fastest a tracer can be advected in the turbulent flow field is the velocity of the field, $V^{*}$. Thus the least possible time it can take tracer to move a distance $L$ is

$$
\mathcal{T}_{\text {advec }}=\frac{L}{V^{*}} .
$$


The time scale of diffusion over the same length scale is

$$
\mathcal{T}_{\text {diff }}=\frac{L^{2}}{A}=\frac{L^{2}}{\gamma V^{*} L^{*}}
$$

This time scale is less than the smallest possible time scale $\mathcal{T}_{\text {advec }}$ when

$$
L<\gamma L^{*}
$$

Thus when the length scales of the tracer are less than the eddy length scales, diffusion over predicts the cross-shelf flux of tracer because it under predicts the time it takes the tracer to move across an eddy. Because of this, diffusive parameterizations like (3.46) are only trustworthy when the length scale of the tracer is greater than an eddy length scale.

Figure 3.23 illustrates the success of (3.46) when the length scales are larger than an eddy length, and the failure when length scales are less than an eddy length. These plots illustrate the evolution of an alongshore uniform bar of tracer placed between 70 and $90 \mathrm{~km}$ offshore in a fully spun up flow field of the base flat bottom case, and compare it to the prediction of that evolution by (3.46). The depth and alongshore averaged tracer concentration are calculated from ten independent realizations of the flow field, which represent an average over $\approx 50$ eddies in the alongshore direction. The snapshots of the depth averaged tracer field are taken from one of these realizations. (3.46) over predicts the diffusion at day 2 after the initialization, but then agrees nicely with the model results at later times. This occurs because the initial distribution is a tophat, with a length scale much less than an eddy lengthscale $(\approx 30 \mathrm{~km})$. Once the length scale is enlarged by mixing, the diffusion parameterization of turbulent mixing is valid. 

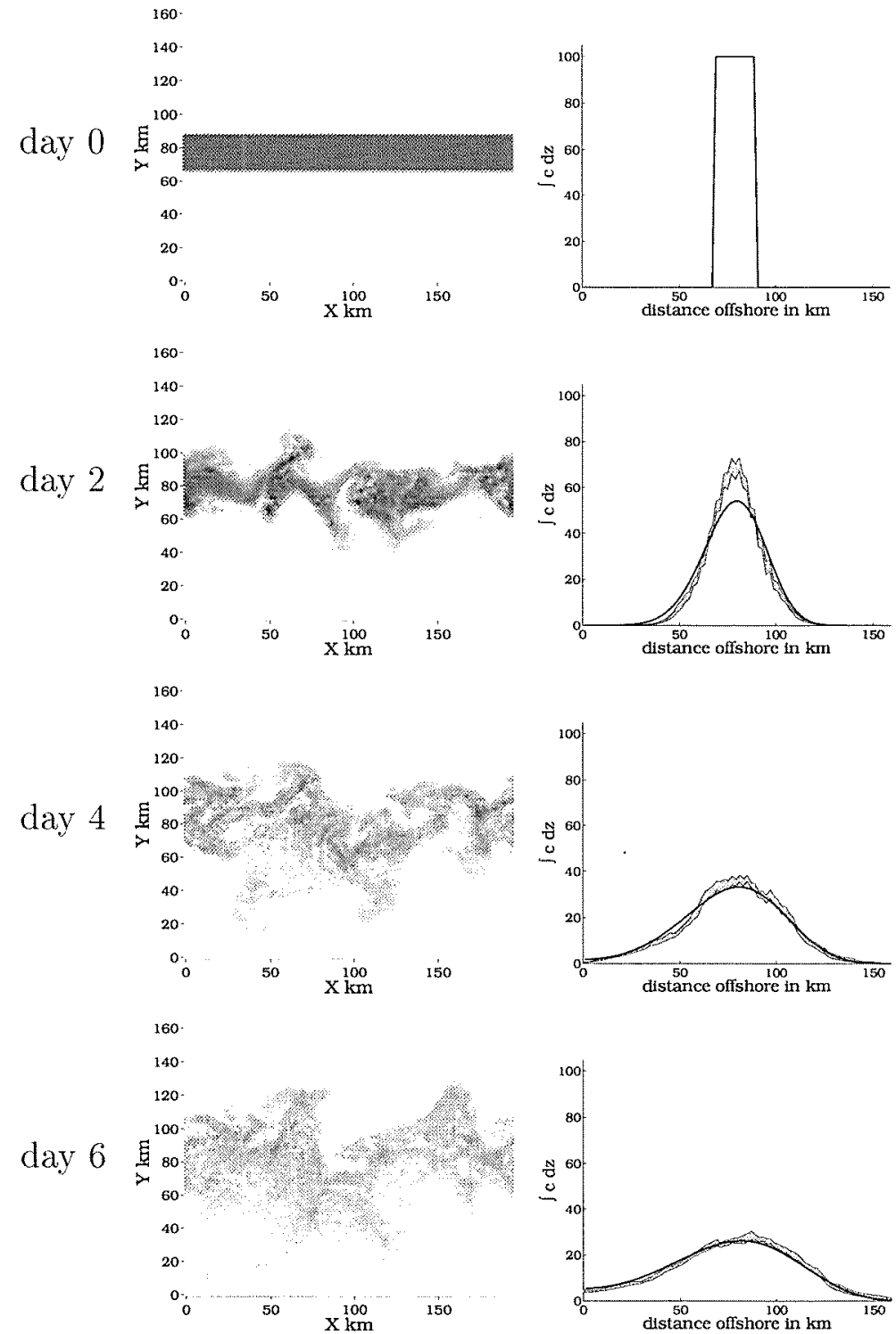

Figure 3.23: The lefthand side is the depth averaged tracer concentration, the righthand side the alongshelf and depth averaged tracer distribution from the diffusion model (3.46) (the solid line) and from the numerical model (the gray region, which is \pm 1 standard error wide). 


\subsection{How do the Cross-Shelf Eddy Heat Fluxes Compare With The Wind Driven Heat Fluxes?}

Throughout this study, the wind driven heat flux across the shelf has been ignored. This could be justified if the heat flux driven by the wind were small compared to the eddy heat flux, and the effect of the wind on the eddies were negligible. The latter is not tested here, but the former can be judged from figure 3.2. The cross-shelf heat flux carried by eddies across the middle of the shelf at $y=80 \mathrm{~km}$ is about one half of the heat flux taken out of the water inshore of $y=80 \mathrm{~km}$ (3.6). The density flux is then $0.25 y_{0} Q$, or $0.28 \mathrm{~kg} \mathrm{~m}^{-1} \mathrm{~s}^{-1}$. The top to bottom density difference at $y=80$ $\mathrm{km}, \Delta \rho$, on day 80 of the model discussed in section 3.2 is about $0.15 \mathrm{~kg} \mathrm{~m}^{-3}$. A downwelling-favorable alongshore wind would force a cross-shelf density flux of

$$
\begin{aligned}
F_{\text {wind }} & =\text { Ekman Transport } \times \Delta \rho \\
& =\frac{\tau^{x}}{\rho_{0} f} \Delta \rho
\end{aligned}
$$

(this assumes thin Ekman layers, but errors in this approximation would only decrease the wind driven density flux). For a $10 \mathrm{~m} \mathrm{~s}^{-1}$ wind, the wind stress is about $10^{-1} \mathrm{~kg} \mathrm{~m}^{-1} \mathrm{~s}^{-1}$, so $F_{\text {wind }}=0.15 \mathrm{~kg} \mathrm{~m}^{-1} \mathrm{~s}^{-1}$, or about half of the eddy driven flux. This supports the idea that eddy driven heat fluxes can be important on the shelf, but are unlikely to always predominate over wind effects. This scaling must be treated with caution, however, because the cross-shelf transport of heat by the wind could alter the stratification upon which the scaling is based. Careful study is needed on the interaction of the winds and eddies. It is conceivable that larger downwellingfavorable alongshore winds could obviate the need for eddy-driven fluxes, but that upwelling-favorable winds will place a greater demand on the eddy fluxes. 


\subsection{Conclusion}

The cross-shelf heat flux driven by a given cross-shelf density gradient is found for a coastal ocean with no mean alongshore variation in forcing or geometry (3.22). It is found under the assumption that the baroclinic instabilities in the flow field have grown to form eddies, and that the cascade of these eddies to larger length scales has been completed. The resulting length scales are estimated with the lesser of the Rhines arrest scale (3.13) or a friction arrest scale (3.19).

This relation between the cross-shelf density gradient and the cross-shelf heat flux is then used to find a scale for the steady-state cross-shelf density gradient of a continental shelf exposed to steady winter-time cooling and a known flux of heat from the deep ocean (3.23). The steady state is attained when the sum of the divergence of the cross-shelf heat flux and the surface cooling is everywhere the same, so that the density of the water increases everywhere at the same rate, leaving the cross-shelf density gradient unchanged (3.6).

The steady-state cross-shelf density gradient scaling is tested in numerical models of the continental shelf run over a broad range of parameters (table 3.1), and it is found that the scaling predicts the cross-shelf density gradient well (figure 3.13). In numerical model runs with geometry and forcing similar to the Mid-Atlantic Bight, the density gradient reaches a steady state in a time comparable with a winter (figure 3.3).

Emboldened by the success of the scaling for the cross-shelf density gradient when the model was run to steady state, the scaling for the cross-shelf flux was written to predict the evolution of the depth averaged density field (3.38). While not strictly valid (for if the density gradient is evolving, the eddy field is not in steady state), (3.38) is found to predict well the evolution of the depth averaged density field in a case where the cooling is suddenly applied near the coast (section $3.7)$.

The results of section 3.7 indicate that the solution to a similar problem by Spall 
and Chapman [1998] is changed by the addition of bottom friction. Bottom friction disrupts the propogation of hetons accross the shelf, thus preventing the density of a cooling region confined near the coast from reaching a steady state.

The eddy mixing scaling is applied to the transport of a passive tracer (3.46), and the parameterization is found to work well once the length scales of the tracer field are larger than an eddy length scale (figure 3.23).

The scales for the cross-shelf transport of heat do not predict the vertical density gradient. Because the vertical density gradient is important for better velocity and length scales (figure 3.14), and is important in predicting the baroclinic stability of the ocean (section 3.7), this is an important deficiency in this scaling.

The effect of ageostrophic wind-driven boundary-layer currents forced by the wind on the eddies has not been considered. Since these currents can be considerable, their effect on the eddies must be considered in any more complete theory.

\section{Acknowledgments}

This paper would have been impossible without Ken Brink's many readings and numerous pieces of scientific advice. Many talks with Mike Spall and Dave Chapman helped to clarify my thinking. Melissa Bowen was kind enough to wade through an early draft. The work was funded by an Office of Naval Research fellowship and and Office of Naval Research AASERT fellowship, N00014-95-1-0746. 


\subsection{Appendix}

The numerical model is SPEM 5.1, an enhanced version of the model described in Hedstrom [1994]. This version of SPEM uses finite differences in the vertical and uses an implicit mixing scheme. The model is now built on a full 3D Arakawa $\mathrm{C}$ grid and is a rigid lid primitive equation model.

The model uses a modified sigma coordinate system in the vertical, in which the vertical resolution near the top and bottom is kept constant while the interior vertical resolution scales with the water depth [Song and Haidvogel, 1994]. The model was run with thirty levels in the vertical, concentrating eight levels in both the top and bottom ten meters in order to resolve the boundary layers. The crossshelf resolution was $2 \mathrm{~km}$, and the alongshelf resolution was between 2 and $4 \mathrm{~km}$, as required to resolve eddies with at least 7 grid-points.

The numerics of the model were changed in order to improve the computational speed by a factor of three for these runs. The implicit vertical mixing scheme is now run at a shorter timestep than the rest of the model. This is necessary because of the extremely high diffusivities needed to model convection. This approach works if the time scale of diffusion, $\Delta z^{2} \nu^{-1}$, is much less than the timescale over which the flow and density fields evolve, which is true for these runs. The general approach used is to write the equations of the model (using the scalar equation as an example) with all but the diffusion terms as forcing terms, e.g.

$$
\rho_{t}-\partial_{z}\left(\nu \partial_{z}(\rho)\right)=\nabla(\vec{u} \rho)+\mathcal{F}_{\rho}
$$

where $\mathcal{F}_{\rho}$ are the physical forcing terms. The left hand side is then computed on a smaller time step assuming the right hand side is a constant, and the right hand side is re-evaluated at the coarser time step. This keeps the solution to the equation well conditioned, keeping numerical noise from corrupting the model. The same implicit Crank-Nicolson scheme is used to evaluate the diffusion equation as originally used 
in SPEM 5.1. The slow time step is 80 seconds and the fast one $262 / 3$ seconds, giving another factor of 3 improvement in speed for the parameter range used below.

The seaward boundary is a free slip vertical wall in the $F_{0}=0$ cases. When the seaward boundary is required to supply an $F_{0}$ capable of balancing the surface cooling, the boundary is modeled with a $15 \mathrm{~km}$ seaward extension to the model domain in which the density is relaxed back to $\rho_{0}$ with a time scale of 3 days. The bottom is flat in this region. The horizontal eddy viscosity in that region is raised to $20 \mathrm{~m} \mathrm{~s}^{-2}$ in the boundary region to dissipate the eddy momentum.

The model was run with the Pacanowski and Philander (1981) Richardson number dependent mixing scheme. This mixing scheme has given good results in previous studies [Allen and Newberger, 1996; Nunes Vas and Simpson, 1994]. The diffusivity and viscosity during stable stratification are given as a function of the gradient Richardson number:

$$
\begin{aligned}
& A=\frac{10^{-2}}{(1+5 R i)^{2}}+10^{-4} \mathrm{~m} \mathrm{~s}^{-2} \\
& \nu=\frac{A}{(1+5 R i)}+10^{-5} \mathrm{~m} \mathrm{~s}^{-2}
\end{aligned}
$$

where

$$
R i=\frac{g \rho_{z}}{\rho_{0}\left(u_{z}^{2}+v_{z}^{2}\right)}
$$

Convective adjustment is handled by an enhanced eddy diffusivity, with a scheme described in chapter 2.

The heat flux at the surface has been converted to a mass flux for the model runs described here. If one assumes a linear equation of state, this is straightforward. Multiplying the boundary condition on temperature

$$
\nu T_{z}=\frac{F}{C_{p} \rho_{0}} \quad(@) z=0
$$




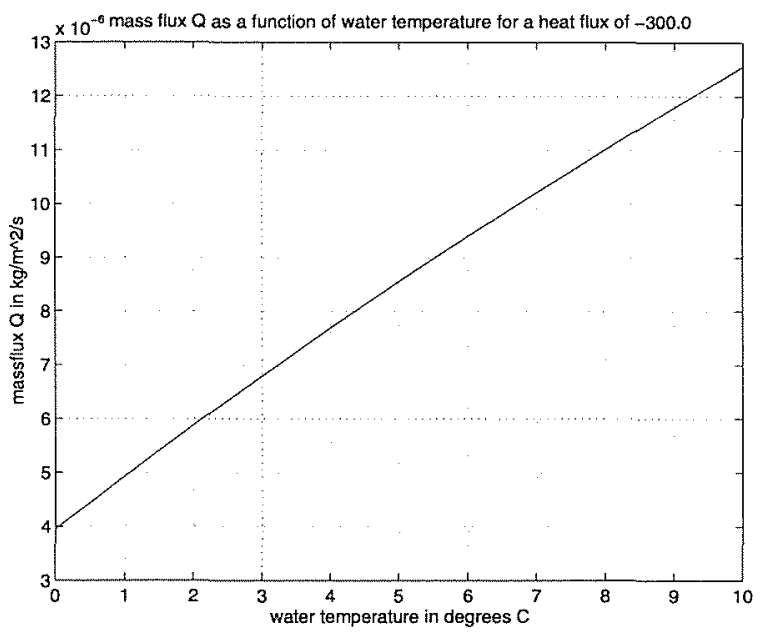

Figure 3.24: This is a plot of the equivalent mass flux for a heat flux of 300 Watts per meter squared as a function of water temperature.

by the thermal expansion coefficient, $\alpha=\frac{\partial \rho}{\partial T}$, gives a boundary condition on density of

$$
\nu \rho_{z}=Q \quad @ z=0
$$

where $F$ is the heat flux in watts per meter squared, and $Q$ the equivalent surface density flux in kilograms per meter squared per second. A problem arises from the fact that $\alpha$ is a strong function of temperature, unlike $\rho_{0}$ and $C_{p}$. For a given heat flux, the equivalent mass flux is 2.2 times greater at $5^{\circ} \mathrm{C}$ than at $0{ }^{\circ} \mathrm{C}$. Figure 3.24 is a plot of the equivalent mass flux for a heat loss of $300 \mathrm{~W} \mathrm{~m}^{-2}$ as a function of water temperature. A mass flux of $7 \times 10^{-6} \mathrm{~kg} \mathrm{~m}^{-2} \mathrm{~s}^{-1}$ is used for the base sloping bottom cases, which is equivalent to a heat loss of $300 \mathrm{~W} \mathrm{~m}^{-2}$ from $3^{\circ} \mathrm{C}$ water or $170 \mathrm{~W} \mathrm{~m} \mathrm{~m}^{-2}$ from $10^{\circ} \mathrm{C}$ water. The surface density flux is fourteen times greater in the base flat bottom cases. 


\subsection{Bibliography}

Allen, J. S. and P. A. Newberger, Downwelling Circulation on the Oregon Continental Shelf. Part I: Response to Idealized Forcing, J. Phys. Oceanogr., 26, 2011-2035, 1996.

Allen, J., Simple Model for Stratified Shelf Flow Fields With Bottom Friction, J. Phys. Oceanogr., 14, 1200-1214, 1984.

Blumsack, S. L. and P. J. Gierasch, Mars: The Effects of Topography on Baroclinic Instability, J. Atmos. Sci., 29, 1081-1089, 1972.

Brown, W. S. and R. C. Beardsley, Winter Circulation in the Western Gulf of Maine: Part I cooling and Water Mass Formation, J. Phys. Oceanogr., 8(2), 265-277, 1978.

Chapman, D. C., Setting the Scales of the Ocean Response to Isolated Convection, J. Phys. Oceanogr., 28, 606-620, 1998.

Chapman, D. C. and G. Gawarkiewicz, Shallow Convection and Buoyancy Equilibration in an Idealized Coastal Polynya, J. Phys. Oceanogr., 27(4), 555-566, 1997.

Condie, S. A. and P. B. Rhines, Topographic Hadley Cells, J. Fluid Mech., 280, 349-368, 1994.

Davis, R. E., Modeling Eddy Transport of Passive Tracers, J. Marine Res., 45, $635-666,1987$.

Dowling, T. E., Dynamics of Jovian Atmospheres, Ann. Rev. Fluid Mech., 27, 293$334,1995$.

Green, J. S. A., Transfer Properties of the Large-Scale Eddies and the General Circulation of the Atmosphere, Quart. J. R. Met. Soc., 96(408), 157-185, 1970. 
Hedstrom, K., SPEM 3.0 User's Guide, Inst. for Naval Oceanography, Technical Report SR-1, 1994.

Held, I. and A. Y. Hou, Nonlinear Axially Symetric Circulation in a Nearly Inviscid Atmosphere, J. Atmos. Sci., 37, 515-533, 1980.

Held, I. M. and V. D. Larichev, A Scaling Theory for Horizontally Homogeneous, Baroclinically Unstable Flow on a Beta Plane, J. Atmos. Sci., 53(7), 946-952, 1995.

Hogg, N. G. and H. M. Stommel, The Heton, an Elementary Interaction Between Discrete Baroclinic Geostrophic Vortices and its Implications Concerning Eddy Heat Flow, Proc. Roy. Soc. Lond., 397, 1-20, 1985.

James, I. N., Introduction to Circulating Atmospheres, Cambridge University Press, New York, 1994.

Killworth, P. D., On the parameterisation of eddy transfer. Part II: Tests with a channel model, J. Marine Res., In Press, 1998.

LaCasce, J. H., Baroclinic Vortices Over a Sloping Bottom, MIT/WHOI Joint Program, PhD Thesis, 1996.

LaCasce, J. H., Geostrophic Turbulence over a Slope: Part II: Potential Vortices, J. Phys. Oceanogr., In submission, 1998.

LaCasce, J. H. and K. H. Brink, Geostrophic Turbulence over a Slope: Part I: Energetics, J. Phys. Oceanogr., In Submission, 1998.

Larichev, V. D. and I. M. Held, Eddy Amplitudes and Fluxes in a Homogeneous Model of Fully Devoloped Baroclinic Instability, J. Phys. Oceanogr., 25, $2285-2297,1995$.

Mountain, D. G., G. A. Stout and R. C. Beardsley, Surface Heat Flux in the Gulf of Maine, Deep Sea Res., 43(II), 1533-1546, 1996. 
Nunes Vas, R. A. and J. H. Simpson, Turbulence Closure Modeling of Esturine Stratification, J. Geophys. Res., 99(C8), 16143-16160, 1994.

Pedlosky, J., Geophysical Fluid Dynamics (second edition), Springer-Verlag, New York, 1987.

Rhines, P. B., The Dynamics of Unsteady Currents, in The Sea, edited by E. A. Goldberg, I. N. McCave, J. J. O'Brien and J. H. Steele, 6, pp. 189-318, Wiley, 1977.

Shepherd, T. G., Nonlinear Saturation of Baroclinic Instability. Part I: The TwoLayer Model, J. Atmos. Sci., 45(14), 2014-2025, 1988.

Song, Y. and D. Haidvogel, A Semi-Implicit Ocean Circulation Model Using a Generalized Topography-Following Coordinate System, J. Comp. Phys., 115, 228$244,1994$.

Spall, M. A. and D. C. Chapman, On the Efficiency of Baroclinic Eddy Heat Transport Across Narrow Fronts, J. Phys. Oceanogr., In Press, 1998.

St-Maurice, J-P. and G. Veronis, A Multi-Scaling Analysis of the Spin-up Problem, J. Fluid Mech., 68, 417-445, 1975.

Stone, P. H., A Simplified Radiative-Dynamical Model for the Static Stability of Rotating Atmospheres, J. Atmos. Sci., 29(3), 405-418, 1972.

Swaters, G. E., On the Baroclinic Instability of Cold-Core Coupled Density Fronts on a Sloping Continental Shelf, J. Fluid Mech., 224, 361-382, 1991.

Visbeck, M., J. Marshall and H. Jones, Dynamics of Isolated convective Regions in the Ocean, J. Phys. Oceanogr., 26, 1721-1734, 1996. 


\title{
Chapter 4
}

\section{High Frequency Linear Internal Waves on a Sloping Shelf}

\begin{abstract}
The behavior of an internal wave in a continuously stratified fluid over a sloping bottom is examined by finding approximate solutions for constant bottom slope, linear bottom friction, and barotropic mean flows. These solutions are valid for frequencies higher than the frequency of critical reflection from the sloping bottom. The solutions show that internal waves propagating towards the shore are refracted so that their crests become parallel to shore as they approach the shore, and outward propagating waves are reflected back toward shore from a caustic. Inviscid solutions predict that the amplitude of a wave goes to infinity at the shore, but these infinite amplitudes are removed by even infinitesimal bottom friction.

These solutions for individual rays are then integrated for an ensemble of internal wave rays of random orientation which originate at the shelf break and propagate across the shelf. It is found that for much of the shelf the shape of the current ellipse caused by these waves is independent of the waves' frequency. The orientation of the current ellipse relative to isobaths is controlled by the redness of the internal wave spectra at the shelf break and the strength of mean currents and current shear. Friction is relatively more important on broader shelves, and consequently on broad shelves the internal wave climate is likely to be dominated by any internal waves generated on the shelf, not waves propagating in from the deep ocean.
\end{abstract}




\subsection{Introduction}

An internal wave propagating obliquely into a coast will turn into the coast, so that its crests will become more parallel to the shore as it moves inwards. A wave propagating obliquely offshore will turn so that its crests become more perpendicular to the shore. This effect, noted by Wunsch [1969] and McKee [1973], will modify the directional spectra of an internal wave field propagating across a shelf, and hence control the high frequency variability on the shelf. The analysis below will examine the evolution of the high frequency internal wave field on the shelf as it is modified by this refraction, mean barotropic currents, and bottom friction.

The study of the cross-shelf evolution of the internal wave field is begun by finding a solution for the amplitude of a progressive, linear internal wave crossing a wedge shaped bathymetry obliquely in the presence of linear bottom friction and barotropic mean alongshore currents. These solutions extend the results of McKee [1973], who gave the solution for an internal wave obliquely crossing an inviscid, quiescent wedge shaped shelf, and Wunsch [1969], who correctly described a progressive internal wave normal to the coast crossing a wedge shaped shelf. Like the solutions of McKee [1973], the solutions derived below are limited to frequencies higher than that of critical reflection from the bathymetry.

The solutions for the propagation of individual internal waves across the shelf are then used to model the evolution of an ensemble of internal waves propagating across the shelf. This ensemble is chosen to resemble the Garrett and Munk [1972] spectrum at a distance offshore chosen to represent the shelf break. It is clearly naive to assume that the spectrum at the shelf break is a Garrett and Munk spectrum [Garrett and Munk, 1972], nevertheless, observations at the shelf break and over the shelf find that the Garrett and Munk spectrum is not a very bad approximation of the internal wave climate near the shelf break (Chapter 5). More detailed modeling of the propagation of internal waves across a shelf break in the limit of a steep shelf break is given in Chapman and Hendershott [1981].

This analysis does not model the effect on internal waves of a baroclinic mean flow, nor does it attempt to explain the non-linear evolution of nearly linear waves due to wave-wave interactions as the waves move onshore. It also ignores the possible effects of alongshore variation in the mean flow and bathymetry. These are quite possibly important effects, and deserve further attention. Nevertheless, the solutions 
derived below can be used as the basis functions for scattering solutions to weakly interacting non-linear problems and the small baroclinic shear problem. The solutions are used to indicate what scales of alongshore variation are important. Because the present analysis does not model the evolution of a wave moving over a topography whose bottom slope is the same as or greater than that needed for critical reflection from the bottom, the following analysis is not valid for near-inertial waves. (For a typical west coast slope of $5 \times 10^{-3}$, the frequency must be at least $5 \%$ greater than $f$ for an $N$ of $100 \mathrm{cpd})$.

In chapter 5, the internal waves observed off the coast of California, USA as part of the 1982 CODE II experiment are analyzed.

\subsection{Plain Internal Wave Solution}

In a flat bottom ocean, the internal wave spectrum can be broken into vertical modes which are orthogonal to each other (e.g. LeBlond and Mysak [1978]). Defining $u$ and $v$ as horizontal velocities, and $w$ as the vertical velocity, the linearized inviscid system of equations on an $f$-plane is:

$$
\begin{aligned}
\frac{\partial u}{\partial t}-f v & =-\frac{1}{\rho_{0}} \frac{\partial P}{\partial \dot{x}} \\
\frac{\partial v}{\partial t}+f u & =-\frac{1}{\rho_{0}} \frac{\partial P}{\partial y} \\
\frac{\partial w}{\partial t} & =-\frac{1}{\rho_{0}} \frac{\partial P}{\partial z}-\frac{\rho g}{\rho_{0}} \\
\frac{\partial u}{\partial x}+\frac{\partial v}{\partial y}+\frac{\partial w}{\partial z} & =0
\end{aligned}
$$

and

$$
\frac{\partial \rho}{\partial t}-\frac{\rho_{0}}{g} N^{2}(z) w=0
$$

$N$ is the buoyancy frequency, $P$ the pressure, $g$ the local gravitational acceleration, $f$ the Coriolis frequency, $\rho_{0}$ the mean water density, and $\rho$ the local deviation of density from $\rho_{0}$. The coordinate system is righthanded with $z$ positive upward. This system 
admits internal wave solutions of the form

$$
\begin{aligned}
u & =\Re\left\{\left(\frac{l f}{\omega}-i k\right) \frac{d W}{d z} e^{i(k x+l y-\omega t)}\right\} \\
v & =\Re\left\{\left(i l-\frac{k f}{\omega}\right) \frac{d W}{d z} e^{i(k x+l y-\omega t)}\right\} \\
w & =\Re\left\{W(z) e^{i(k x+l y-\omega t)}\right\}
\end{aligned}
$$

where $\phi$ is an arbitrary phase, $k$ the horizontal wave number, $\omega$ the angular frequency, and $W(z)$ the vertical modal structure. $W(z)$ and $k$ are determined for a given $\omega$ by

$$
\begin{gathered}
\frac{d^{2} W}{d z^{2}}+\left(k^{2}+l^{2}\right)\left(\frac{N^{2}(z)-\omega^{2}}{\omega^{2}-f^{2}}\right) W=0, \\
w=0 \text { at the top and bottom. }
\end{gathered}
$$

If $N$ is independent of depth, $W$ has the form

$$
W=\sin \left(\frac{M \pi}{D} z\right) \quad M=1 \ldots \infty,
$$

where $D$ is the water depth and $M$ the mode number. When $N$ varies with depth, (4.3) must usually be solved numerically and the modal structure is no longer independent of $\omega$. Nonetheless, the structure does not vary strongly with changes in $\omega$ or with changes in the $N$ profile as long as $\omega^{2} \ll N^{2}$. This is because any change in

$$
\frac{N^{2}(z)-\omega^{2}}{\omega^{2}-f^{2}}
$$

only affects the solution to the extent that (4.5) varies from its depth averaged value. Any constant multiplicative change to its value is absorbed into the eigenvalue $k^{2}+l^{2}$ and does not affect $W(z)$. To illustrate this, the first and second vertical modes for two different frequencies, 2 and $40 \mathrm{cpd}$, are calculated for both a constant $N$ and an $N$ which varies realistically from $100 \mathrm{cpd}$ at the surface to $50 \mathrm{cpd}$ at the bottom (figure 4.1. This is the average profile used in chapter 5.) The modes are plotted in figure 4.2 , and it can be seen that neither changes in frequency nor a depth-dependent $N$ affect the modal structure very much. Because of this, there are only small differences in the dispersion relation for a constant $N$ and the dispersion relation for a variable $N$, as long as $\omega^{2} \ll N^{2}$. Thus, in the following, a constant $N$ is used that corresponds to the depth averaged $N$ of a real profile.

The modal solutions suppose a flat bottom in their bottom boundary condition. The true boundary condition for a sloping bottom is that the velocity normal to 


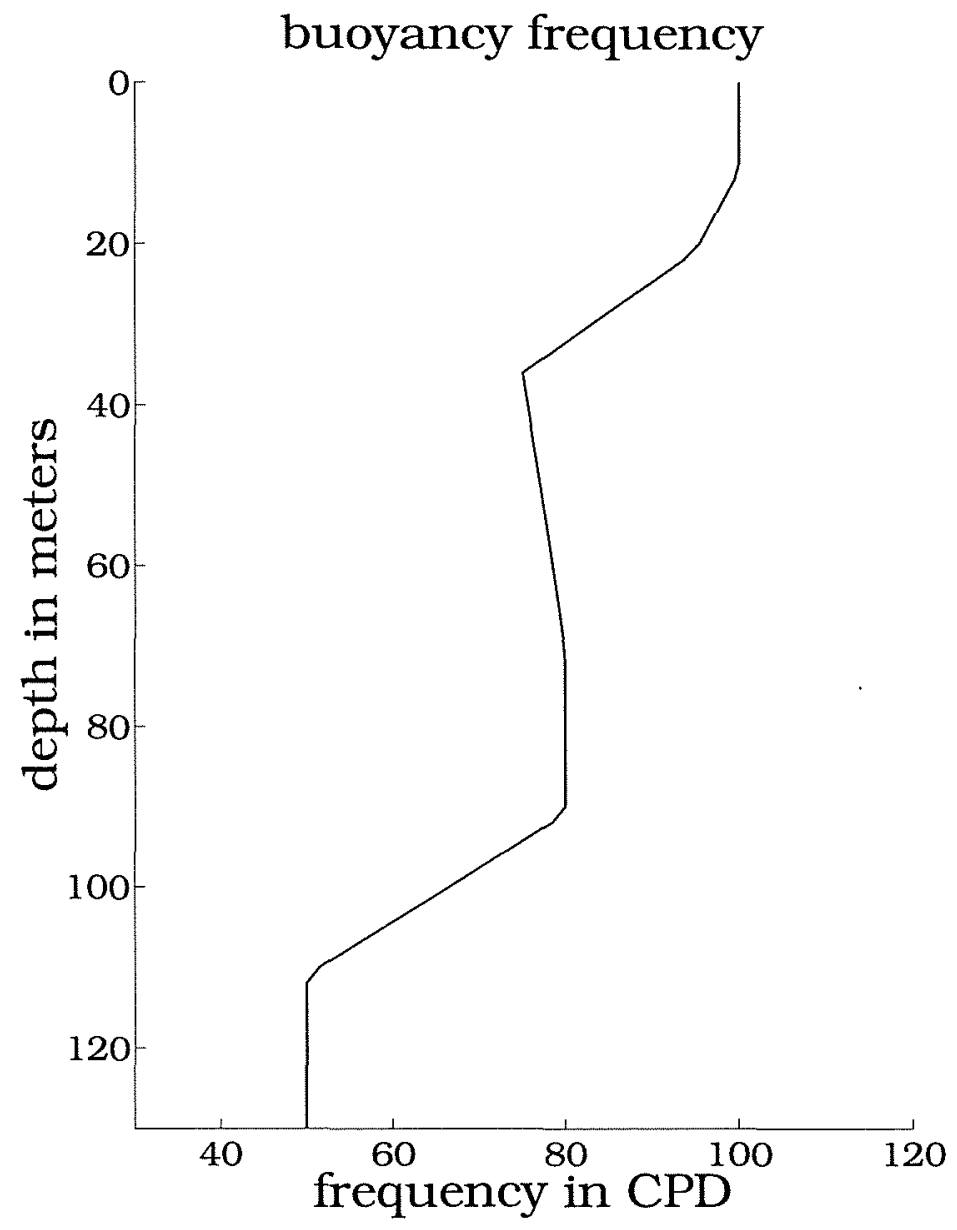

Figure 4.1: The average buoyancy profile for July, 1982 at 130 meters depth in the CODE-II region of coastal California, USA. (Chapter 5) 

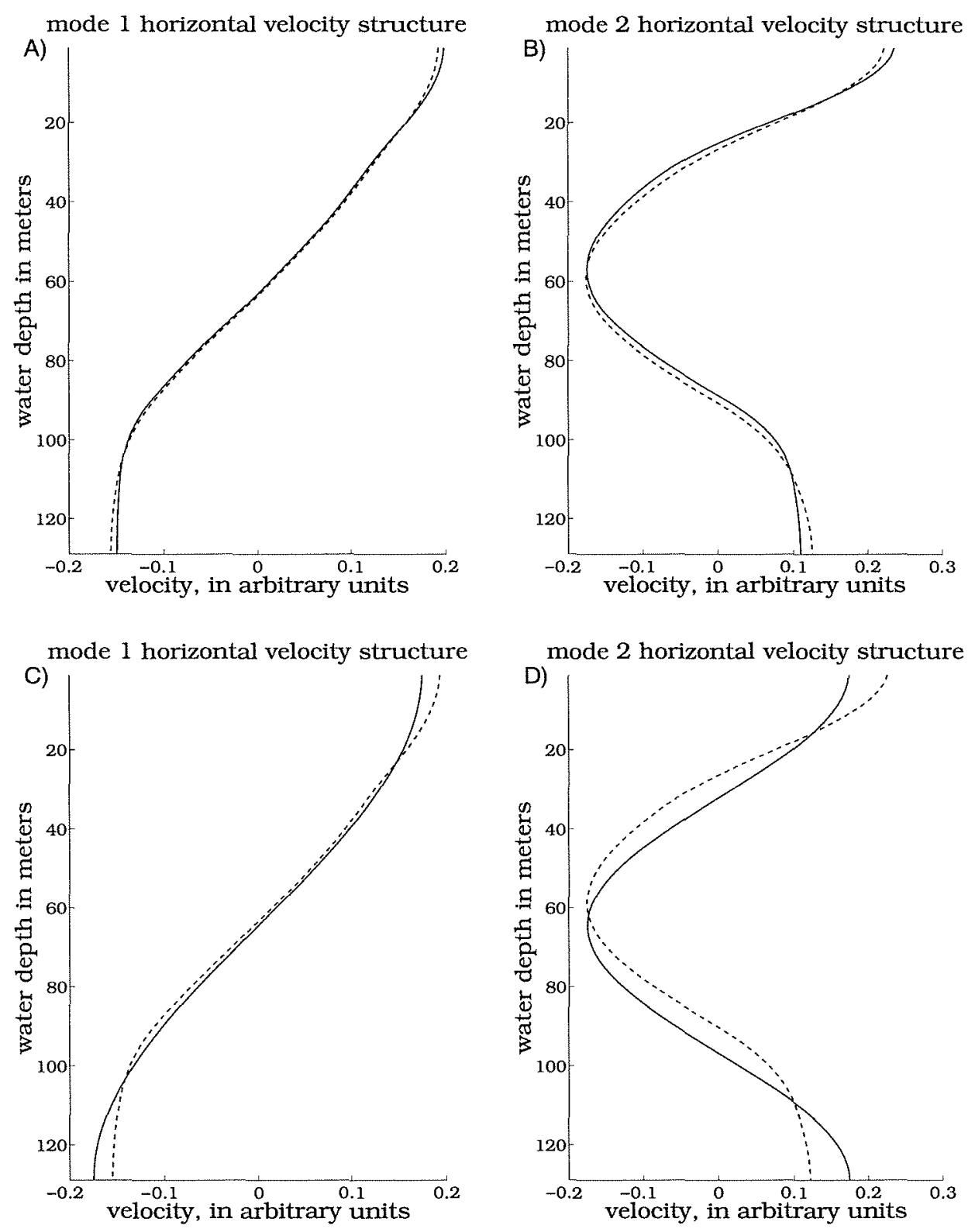

Figure 4.2: The top two panels are of mode 1, on the left, and mode 2, on the right. The dashed line is for $\omega=2 \mathrm{cpd}$, and the solid line is $40 \mathrm{cpd}$. The $N$ in the top panel is from figure 4.1. The bottom two panels plot the same modes for $N$ constant (solid line) and the $N$ profile in figure 4.1 (dashed line) for $\omega=10 \mathrm{cpd}$. 
the boundary be zero. Wunsch [1969] shows that the solution over a wedge-shaped topography limits to the flat bottom result when the slope of the wave characteristics, $c$,

$$
c=\sqrt{\frac{\omega^{2}-f^{2}}{N^{2}-\omega^{2}}},
$$

becomes more then twice the bottom slope $\alpha$. (This can be most easily seen by directly comparing equations (8) and (12) of Wunsch [1969]. The error in using the flat-bottom modal solution is less than $10 \%$ when $0.5 c>\alpha$.) The criterion for the validity of vertical modes can thus be obtained by solving equation (4.6) for frequency, and substituting twice the bottom slope for the characteristic wave slope, leading to:

$$
\omega^{2} \gg f^{2}+4 \alpha^{2} N^{2}
$$

For an $f$ of $1.24 \mathrm{cpd}$, and an $N$ of $100 \mathrm{cpd}$, this is true for frequencies $28 \%$ higher than $f$ for a slope of $5 \times 10^{-3}$, and $90 \%$ higher than $f$ for a slope of $10^{-2}$. This frequency criterion is equivalently the condition for avoiding critical reflection off the bottom, thus any wave whose vertical structure is well described by (4.3) is not subject to critical reflection from the bottom. The relation between these two facts is explored in Wunsch [1969].

\subsection{The Path of a Wave with No Mean Flow}

The first step in understanding the propagation of internal waves on the shelf is to study the behavior of a single plane wave on a shelf with no mean flow, constant $N$, and no alongshore variability in topography.

Basic ray tracing theory (e.g. Lighthill [1978]) states that if there is no variation of $N$ or $D$ in the alongshore direction, $l$ is conserved along a ray, and if the mean currents do not vary with time, the observed frequency $\omega$ is conserved along a ray. From (4.3) the dispersion relation for the modal internal waves in the absence of mean flow is, for $\alpha c^{-1} \ll 1$,

$$
\omega=\sqrt{\frac{\left(k^{2}+l_{0}^{2}\right) N^{2}+f^{2} \frac{M^{2} \pi^{2}}{D^{2}}}{k^{2}+l_{0}^{2}+\frac{M^{2} \pi^{2}}{D^{2}}}},
$$

where $l_{0}$ is the conserved alongshore wave number. Given a depth, $D_{0}$, and a cross shelf wave number, $k_{0}$, at any point on the ray path, one can find the cross shelf 
wavenumber, $k_{m}$, at any other point on the ray path where one knows the depth $D_{m}$

$$
k_{m}^{2}=\frac{D_{0}^{2}}{D_{m}^{2}}\left(k_{0}^{2}+l_{0}^{2}\right)-l_{0}^{2}
$$

(Throughout the following, the subscripts $m$ and 0 will be used denote properties of waves at two locations along the same ray.)

Because the internal wave dispersion relation (4.8) depends only on the horizontal wave vector magnitude, not its direction, the group velocity is parallel to the wave vector. It will often be useful to consider the angle the wave vector, and thus the ray path, makes with the onshore direction. This angle, $\theta$, is

$$
\theta=\arctan \left(\frac{l}{k}\right)
$$

and can be related to the depth and angle at some point with the depth at another point by substituting (4.9) into (4.10) to get

$$
\theta_{m}=\arctan \left(\frac{\sin \left(\theta_{0}\right)}{\sqrt{\frac{D_{0}^{2}}{D_{m}^{2}}-\sin ^{2}\left(\theta_{0}\right)}}\right) .
$$

Several important phenomena are revealed by (4.11) and (4.9). As $D_{m}$ decreases, $k_{m}$ increases, and so the angle the ray makes to the cross shelf direction decreases. Thus the ray path turns towards shore as the water gets shallower. Conversely, as long as $l_{0}^{2} \neq 0$ there is always some depth $D_{m}>D_{0}$ where $k \rightarrow 0$. This could be naively interpreted as the point where $\theta_{0}=90^{\circ}$, and the wave moves straight along an isobath. However, ray tracing theory breaks down when the cross shelf wavelength, $2 \pi k^{-1}$, exceeds the topographic length scale $D\left(\frac{\partial D}{\partial x}\right)^{-1}[$ Lighthill, 1978]. As appendices $A$ and $B$ detail, the ray tracing assumption remains valid until very near the location where $k$ becomes zero, at which point the energy in the wave is reflected back to shore, with $\omega$ and $l_{0}$ unchanged.

To analyze the behavior of the wave further, a specific bathymetry, $D(x)$, must be specified. For the sake of simplicity, a linear wedge-shaped topography is chosen, with the coast at $x=0$, and the sea extending to $x=-\infty$. The depth is $D=-\alpha x$. None of the results below are qualitatively affected by the choice of depth profile, as long as the bottom slope remains finite as one approaches the shore.

The ray tracing theories presented here correspond to the solutions in equation 37 of Wunsch [1969] as long as $\alpha^{-1} c \gg 1$, but are easier to work with than his Bessel 
functions of large, complex, and non-integer order. The ray theory solution allows one to trace energy propagation more easily and understand the effects of friction more readily than Wunsch's wave modes allow.

Since the group velocity is in the direction of the wave vector, and since the group velocity defines the ray path, the equation of the ray path is

$$
\frac{d y}{d x}=\frac{d y / d t}{d x / d t}=\frac{\overrightarrow{c_{g}} \cdot \hat{j}}{\overrightarrow{c_{g}} \cdot \hat{i}}=\frac{l_{0}}{k_{m}} .
$$

Equation (4.9) can be substituted for $k_{m}$ and (4.12) integrated to get the ray path

$$
y= \pm x_{0} \sqrt{\frac{k_{0}^{2}}{l_{0}^{2}}+1-\frac{x^{2}}{x_{0}^{2}}}+\left(-\frac{k_{0}}{l_{0}} x_{0}+y_{0}\right)
$$

The wave can move in either direction along this path. If $x_{0}$ is chosen as the location where $k$ would vanish, " $-x_{c}$ ", and $y_{0}$ is taken as zero, the solution has the very simple form

$$
y= \pm x_{c} \sqrt{1-\frac{x^{2}}{x_{c}^{2}}}
$$

where, from (4.8)

$$
x_{c}=\frac{M \pi c}{\alpha l_{0}} .
$$

This path is a circle whose radius is $x_{c}$. The path is plotted in figure 4.3 with the axes scaled by $x_{c}$. The ray path in (4.14) defines the path along which the internal wave energy will propagate as long as ray tracing remains valid. As is shown in appendix $B$, ray tracing remains valid except near $-x_{c}$. Ray tracing is not valid when

$$
\frac{\left(x+x_{c}\right)}{x_{c}} \leq 2^{-\frac{1}{3}}\left(\frac{M \pi c}{\alpha}\right)^{-\frac{2}{3}} .
$$

Since $c \alpha^{-1} \gg 1$ is the condition for the validity of vertical modes, the region where ray tracing fails is a small portion of the region to which the wave is trapped. For a mode 1 wave with $\omega=10 \mathrm{cpd}, N=100 \mathrm{cpd}, f=1.24 \mathrm{cpd}$, and a bottom slope of $5 \times 10^{-3}$, ray tracing is valid for $95 \%$ of the distance between the shore and $-x_{c}$. The location of the caustic is defined by (4.16), and the energy in the wave is reflected back to the coast from this region. Thus $-x_{c}$ marks the point past which the energy 
density falls rapidly to zero. This is examined in more detail in appendix $\mathrm{B}$. The only way a wave with $l \neq 0$ can travel farther offshore than $x_{c}$ is for the vertical modal structure to break down, or for the mode to "stop feeling" the bottom. The vertical mode assumption can break down if the bottom slope exceeds the critical value in (4.6). Alternatively the vertical mode can cease to feel the bottom, and hence cease to be steered by the topography, if the stratification $N$ near the bottom falls below $\omega$. Likewise, waves propagating onshore from the deep ocean will only begin to be governed by (4.14) when $N>\omega$ near the bottom and the bottom slope is less than the critical slope. Offshore of either of these points, topography will no longer govern the ray paths.

The derivation above assumes that there is no alongshore variation in the bathymetry. (4.14) shows that a wave trapped inside of $x=-x_{c}$ will travel a distance of $2 x_{c}$ along the coast before the ray intersects the coast. This implies that alongshore variation has to be small over a distance of $2 x_{c}$ for these derivations to be valid. Thus a shelf can be considered uniform in the alongshelf direction if the bathymetry and mean currents do not vary much over an alongshore distance comparable to the distance between the shelf break and the shore.

The wavenumber vector $\left(k_{0}, l_{0}, M\right)$ at a point defines an internal wave and its ray path, as does $(\theta, \omega, M)$ at a point. The quantities $\left(l_{0}, \omega, M\right)$ also define a wave, and remain the same everywhere along the wave's ray path. However, in the next several sections, $\left(x_{c}, \omega, M\right)$ will be used to label waves because $x_{c}$ can define a ray path alone. All the labels are equivalent and (4.9), (4.11) and (4.8) can be used to switch among them.

\subsection{Wave Amplitude: the Inviscid Problem}

A wave traveling in the absence of a mean current in an inviscid ocean conserves its energy, and the energy is carried at the wave's group velocity $\overrightarrow{c_{g}}[$ Lighthill, 1978, p321]. This means that the conservation of the areal energy density can be expressed as

$$
\vec{\nabla} \cdot\left(\vec{c}_{g} E\right)=0
$$




\section{internal wave ray path}

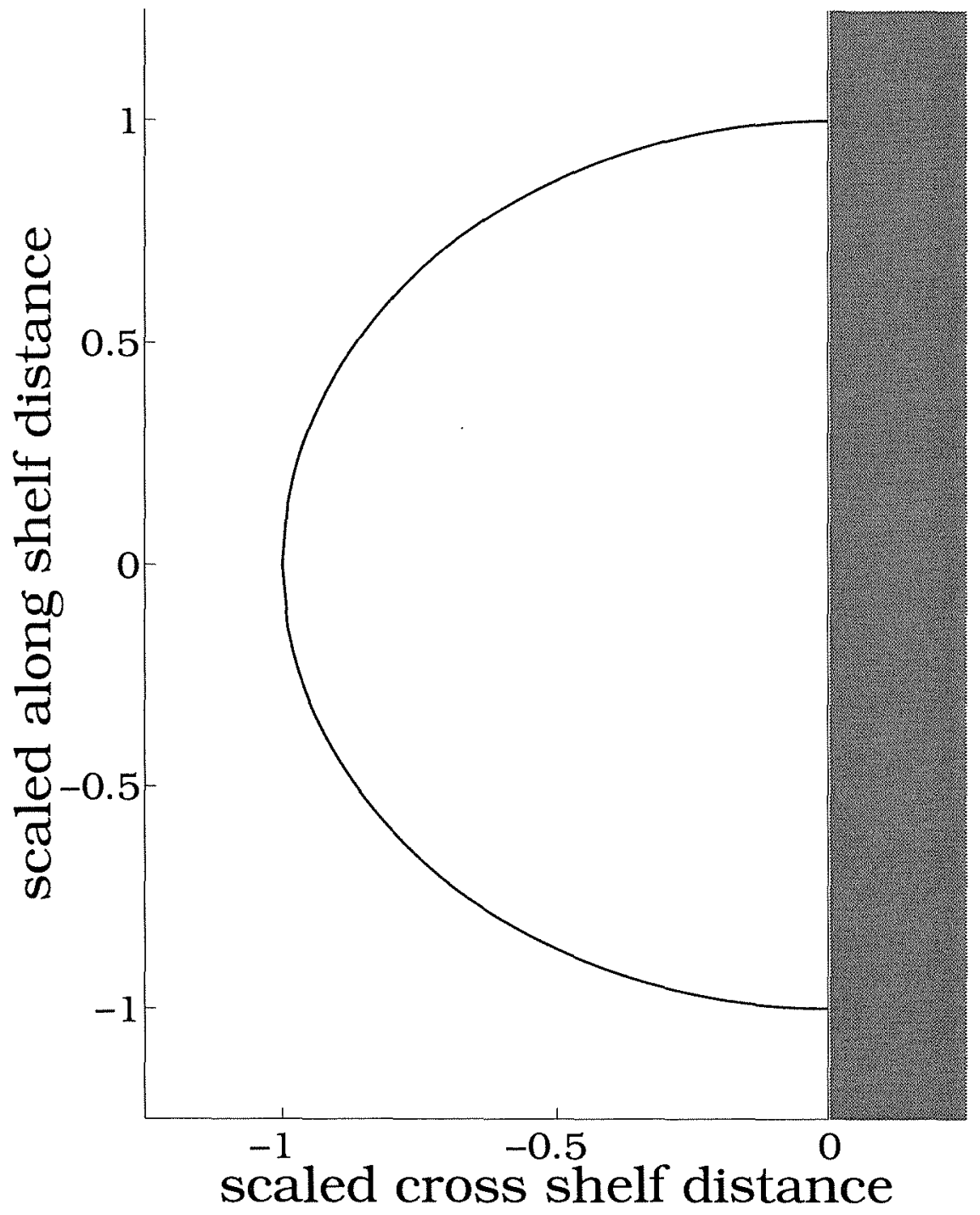

Figure 4.3: The path of an internal wave over a sloping bottom of constant slope in the absence of mean flows. The distance alongshore and cross shore have both been normalized by $x_{c}$ 
It is easier to work with the depth-averaged volume energy density $\left\langle E_{d}\right\rangle$ of the wave

$$
\left\langle E_{d}\right\rangle=\frac{1}{4} \rho_{0} A_{h}^{2}\left(\frac{N^{2}-f^{2}}{N^{2}-\omega^{2}}\right)=\frac{W_{0}^{2} \rho_{0} N^{2}}{4 \omega^{2}},
$$

where $W_{0}$ is the amplitude of the vertical velocity mode (4.3c), and $A_{h}$ is the amplitude of the horizontal velocity mode (4.3a). Using the depth averaged volume energy density, (4.17) becomes

$$
\vec{\nabla} \cdot\left(\overrightarrow{c_{g}} D\left\langle E_{d}\right\rangle\right)=0
$$

where $D$ is the water depth. Since there is no variation in the alongshore direction (4.19) can be written as

$$
\frac{\partial}{\partial x}\left(\overrightarrow{c_{g}} \cdot \hat{i} D\left\langle E_{d}\right\rangle\right)=0 .
$$

This can also be expressed in terms of the magnitude of the group velocity and the angle of the wave vector to the onshore direction:

$$
\left|\overrightarrow{c_{g}}\right| \cos (\theta) D\left\langle E_{d}\right\rangle=\text { constant }
$$

The group velocity magnitude can be found by taking the wave number derivative of (4.8) and using (4.9) to find the local $k$ in terms of the water depth $D$. This yields

$$
\left|\overrightarrow{c_{g}}\right|=S D
$$

and

$$
S=\frac{c\left(N^{2}-f^{2}\right)}{M \pi\left(1+c^{2}\right)^{\frac{3}{2}}\left(c^{2} N^{2}+f^{2}\right)^{\frac{1}{2}}},
$$

where $\mathrm{S}$ is a constant with units of time $\mathrm{e}^{-1}$ and $c$ is as defined by (4.6).

Since the wave is inviscid, the energy density only depends on the depth of the water and the initial amplitude and orientation of the ray, as can be seen from (4.21) and (4.11). Thus a wave which had at depth $D_{0}$ an angle of $\theta_{0}$ and an energy density of $\left\langle E_{d}\right\rangle_{0}$ will have an energy density of

$$
\left\langle E_{d}\right\rangle_{m}=\left\langle E_{d}\right\rangle_{0} \frac{D_{0}^{2}}{D_{m}^{2}} \frac{\cos \left(\theta_{0}\right)}{\sqrt{1-\frac{D_{2}^{2}}{D_{0}^{2}} \sin ^{2}\left(\theta_{0}\right)}}
$$


when it reaches water of depth $D_{m}$. However, to be more consistent with the following analysis, the energy density can be cast in terms of $x_{c}$ and position $x$ with the assumption of a wedge shaped topography. Thus

$$
\left\langle E_{d}\right\rangle=\frac{\mathcal{C}}{x^{2} \sqrt{1-\frac{x^{2}}{x_{c}^{2}}}}
$$

where $\mathcal{C}$ is a constant.

From (4.23) and (4.24), it can be seen that as $D_{m} \rightarrow 0,\left\langle E_{d}\right\rangle$ grows as $D_{m}^{-2}$. Equivalently, for the constant bottom slope case of (4.24), as $x \rightarrow 0,\left\langle E_{d}\right\rangle$ grows as $x^{-2}$. This amplification has two causes. First, from (4.21), as the water depth decreases the volume energy density for a given areal energy density increases as $D^{-1}$. Second, from (4.22), the magnitude of the group velocity decreases with $D$, and so the areal energy density has to increase as $D^{-1}$ to keep the energy flux constant. However, as will be shown in the next section, even the smallest amount of friction will limit the magnitude of $\left\langle E_{d}\right\rangle$ in shallow water, and $\left\langle E_{d}\right\rangle$ will fall to zero at the coast.

From (4.24) it appears that as $x \rightarrow-x_{c}$, the energy density increases to infinity because, as the wave vector, and hence the group velocity, turns to parallel the coast, the areal energy density must increase to keep the product of the cross shelf group velocity with the areal energy density a constant. However, as appendix B shows, the amplitude of the wave only increases until ray tracing theory breaks down in the caustic, at which point the amplitude falls rapidly to zero as the energy is reflected back to the coast.

\subsection{Wave Amplitude: the Frictional Problem}

The presence of friction in the ray tracing problem has several important ramifications. The first is that while the path of the ray may still be reversible, the wave's amplitude is no longer independent of the direction of propagation: the total wave energy flux always decreases in the direction of propagation. Further, as mentioned above, the addition of friction also keeps the energy density bounded as the wave approaches the coast. 
The frictional analysis is based on Brink [1988], who analyzed the effect of a linear bottom drag law

$$
\tau_{\text {bottom }}=\rho_{0} r \vec{u}_{\text {bottom }}
$$

and assumed that the friction is weak, i.e. that the time scale of friction $D / r$ is much greater then the time scale of the wave $\omega^{-1}$. The assumption that friction is weak means that the group and phase velocities are unchanged from the inviscid case. The restriction that

$$
\frac{r}{D} \ll \omega
$$

remains valid for a reasonable value of $r, r=5 \times 10^{-4} \mathrm{~m} \mathrm{~s}^{-1}$, and $\omega, 10 \mathrm{cpd}$, until the water is only five meters deep. A comparison of more complicated drag laws with the linear law is given in Sanford and Grant [1987], while a discussion of the validity of Brink [1988], along with an extension and re-derivation of the result, is given in appendix $\mathrm{A}$.

Brink [1988] shows that the energy density of a plane wave in constant stratification over a flat bottom decays as

$$
\left\langle E_{d}\right\rangle=\left\langle E_{d}\right\rangle_{0} \exp \left[-2 \frac{r}{D}\left(1+\frac{f^{2}}{\omega^{2}}\right) t\right] .
$$

The time scale of energy decay, $T$, will be approximated here as

$$
T=\frac{D}{2 r}+O\left(\frac{f^{2}}{\omega^{2}}\right) \text {. }
$$

To integrate this into a ray tracing model, it is necessary to include this energy sink term in equation (4.17):

$$
\frac{D_{\overrightarrow{c_{g}}}}{D t} D\left\langle E_{d}\right\rangle=-T^{-1}\left\langle E_{d}\right\rangle D
$$

or

$$
\frac{\partial}{\partial x}\left(\overrightarrow{c_{g}} \cdot \hat{i} D\left\langle E_{d}\right\rangle\right)=-T^{-1}\left\langle E_{d}\right\rangle D .
$$

By assuming a water depth of the form $D=-\alpha x$ this can be written in terms of the distance offshore using equations (4.22) and (4.11).

$$
\frac{\partial}{\partial x}\left(x^{2} \sqrt{1-\frac{x^{2}}{x_{c}^{2}}}\left\langle E_{d}\right\rangle\right)= \pm \frac{2 r}{S \alpha^{2}}\left\langle E_{d}\right\rangle
$$


The right hand side is positive when the wave is propagating offshore, and negative when the wave is propagating towards the coast. Equation (4.31) can be solved to obtain

$$
\left\langle E_{d}\right\rangle=\mathcal{C} \frac{\exp \left(\frac{2 r}{S \alpha^{2}} \frac{\sqrt{1-\frac{x^{2}}{x_{c}^{2}}}}{x}\right)}{x^{2} \sqrt{1-\frac{x^{2}}{x_{c}^{2}}}}
$$

for a wave traveling onshore and

$$
\left\langle E_{d}\right\rangle=\mathcal{C} \frac{\exp \left(-\frac{2 r}{S \alpha^{2}} \frac{\sqrt{1-\frac{x^{2}}{x_{c}^{2}}}}{x}\right)}{x^{2} \sqrt{1-\frac{x^{2}}{x_{c}^{2}}}}
$$

for a wave traveling offshore. These solutions contain only two length scales, $x_{c}$ and $2 r S^{-1} \alpha^{-2}$, so the solution is controlled by these two length scales. The length scale $K \equiv 2 r S^{-1} \alpha^{-2}$ is not the frictional length scale. The friction length scale, the distance defined by the group velocity times the friction time scale $2^{-1} r^{-1} D$, is

$$
L_{f}=\frac{S D^{2}}{2 r}=\frac{x^{2}}{K} .
$$

The ratio of $L_{f}$ to $x_{c}$, averaged inshore of $x_{c}$, is thus

$$
\frac{L_{f}}{x_{c}}=\frac{x_{c}}{3 K}
$$

When this ratio is large, the wave can travel over a distance of $x_{c}$ without being dissipated. If the ratio is small, dissipation claims the wave before it can travel a distance comparable to $x_{c}$. Thus if $x_{c} \gg K$ the wave is little affected by friction, and if $x_{c} \ll K$ the wave is frictionally dominated. To illustrate this, the evolution of $\left\langle E_{d}\right\rangle^{\frac{1}{2}}$ has been computed for a wave which originates at $-0.5 x_{c}$, travels to $-x_{c}$, and is reflected back to the coast. The path of this wave is plotted in figure 4.4. For simplicity, the reflection at the caustic is assumed here to occur without significant loss of energy, which is not always the case (appendix B covers the caustic in more detail). $\left\langle E_{d}\right\rangle^{\frac{1}{2}}$ is plotted in figure 4.5 for the inviscid, $x_{c} \gg K, x_{c} \approx K$, and $x_{c} \ll K$ cases. $\left\langle E_{d}\right\rangle^{\frac{1}{2}}$ is proportional to the velocity amplitude of the wave. For the inviscid case (figure $4.5 \mathrm{a}$ ), the wave has the same energy leaving and approaching the coast, 
and $\left\langle E_{d}\right\rangle^{\frac{1}{2}}$ goes to infinity as the wave approaches the coast. However, even with weak friction, $x_{c} \gg K,\left\langle E_{d}\right\rangle$ falls to zero as the wave approaches the coast. For both the $x_{c} \approx K$ and $x_{c} \ll K$ cases, $\left\langle E_{d}\right\rangle$ always decreases after being reflected at $x_{c}$. Friction causes $\left\langle E_{d}\right\rangle$ to decay rapidly before the wave reaches the coast because the group velocity decreases linearly with the depth, and thus the wave takes infinitely long to reach the coast for any bottom topography whose slope remains finite and non-zero as the shore is approached. Since bottom friction has a finite time scale, the wave will be dissipated before it reaches the coast. Because of this, wave breaking or other nonlinear effects are not required to explain the disappearance of the internal waves as they propagate towards the coast. Further, when $K \geq x_{c},\left\langle E_{d}\right\rangle$ decays monotonically as the wave moves to the coast and so internal waves are unlikely to break if $K \geq x_{c}$.

It should be noted that the effect of friction on internal waves in a continuously stratified fluid is fundamentally different than the response of surface gravity waves or interfacial waves in a two layer system. Linear surface gravity waves and internal waves on the interface of a two layer fluid reach the beach before being entirely dissipated. This is because the latter waves have group velocities that depend on the square root of the water depth, instead of linearly with the water depth, and thus reach the shore in a finite time. This difference is not caused by a fundamental difference in the physics of gravity waves. Long surface gravity waves, long interfacial waves, and internal gravity waves all have group velocities that scale with the square root of the top to bottom (or air/water) density difference and the square root of the water depth. It is just that for an internal wave in a continuously stratified ocean, the top to bottom density difference scales as the water depth, and thus the group velocity scales as depth.

For a typical buoyancy frequency of $100 \mathrm{cpd}$ and an $r$ of $5 \times 10^{-4} \mathrm{~m} \mathrm{~s}^{-1}$, the frictional length scale $\mathrm{K}$ is of order 20 to $30 \mathrm{~km}$ for a mode 1 wave of $5-70$ cycles per day over a typical United States west coast slope of $5 \times 10^{-3}$. For the same waves on a typical United States east coast slope of $1 \times 10^{-3}$ the length is 25 times longer, of order 500 to $750 \mathrm{~km}$, and thus friction more completely attenuates waves during their passage over the broader east coast shelf. The frictional length scale increases as the frequency increases because, as seen in figure 4.6, the group velocity, and hence $S$, decrease as frequency increases. However, the fractional increase is order one until the frequency reaches $0.7 N$, after which it increases rapidly. 


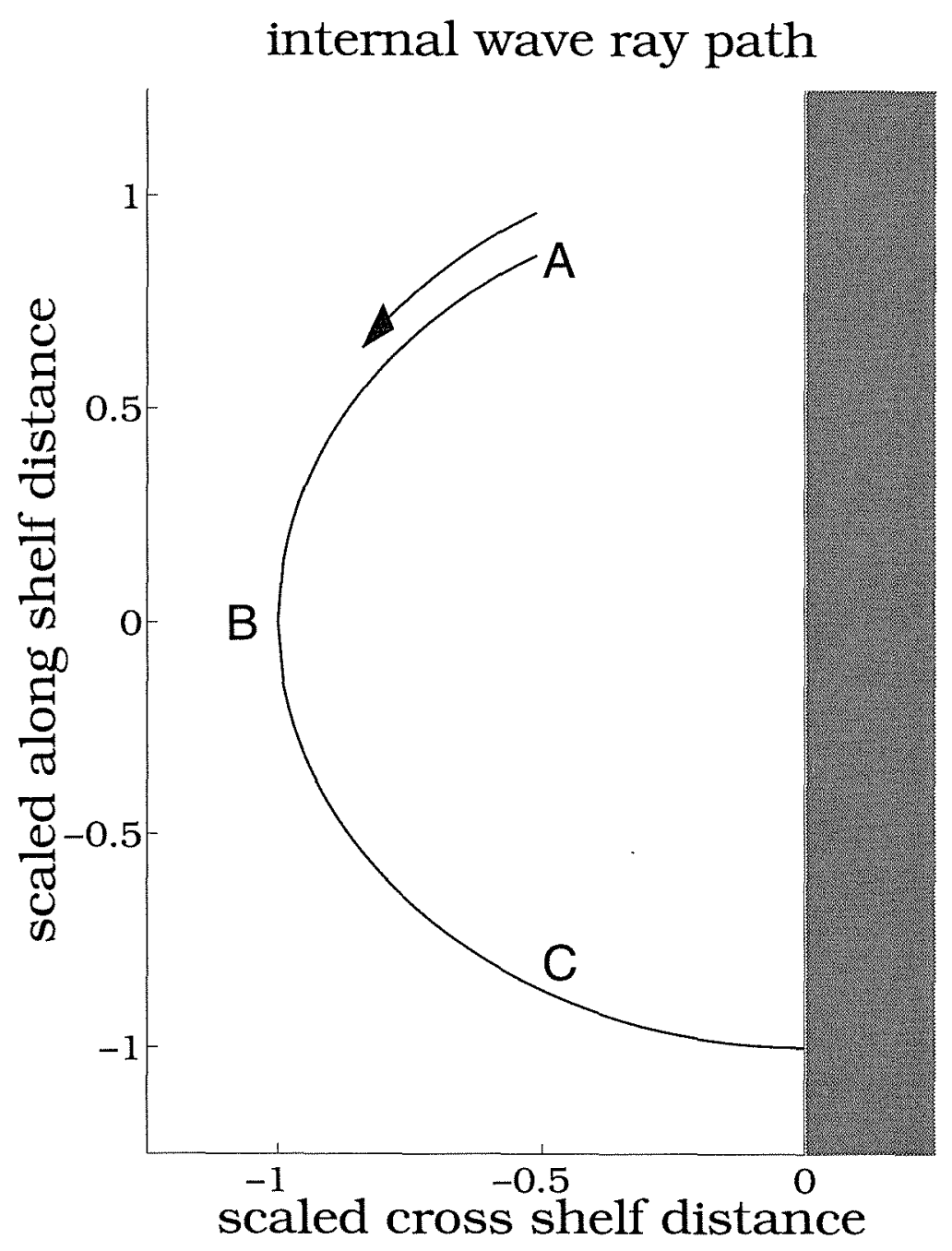

Figure 4.4: The path of an internal wave that starts at the point marked A, propagates offshore, is reflected back to shore at the caustic marked $B$, and continues into the shore past the point marked $\mathrm{C}$. Both axes have been normalized by the distance offshore of the point of reflection, $x_{c}$. 

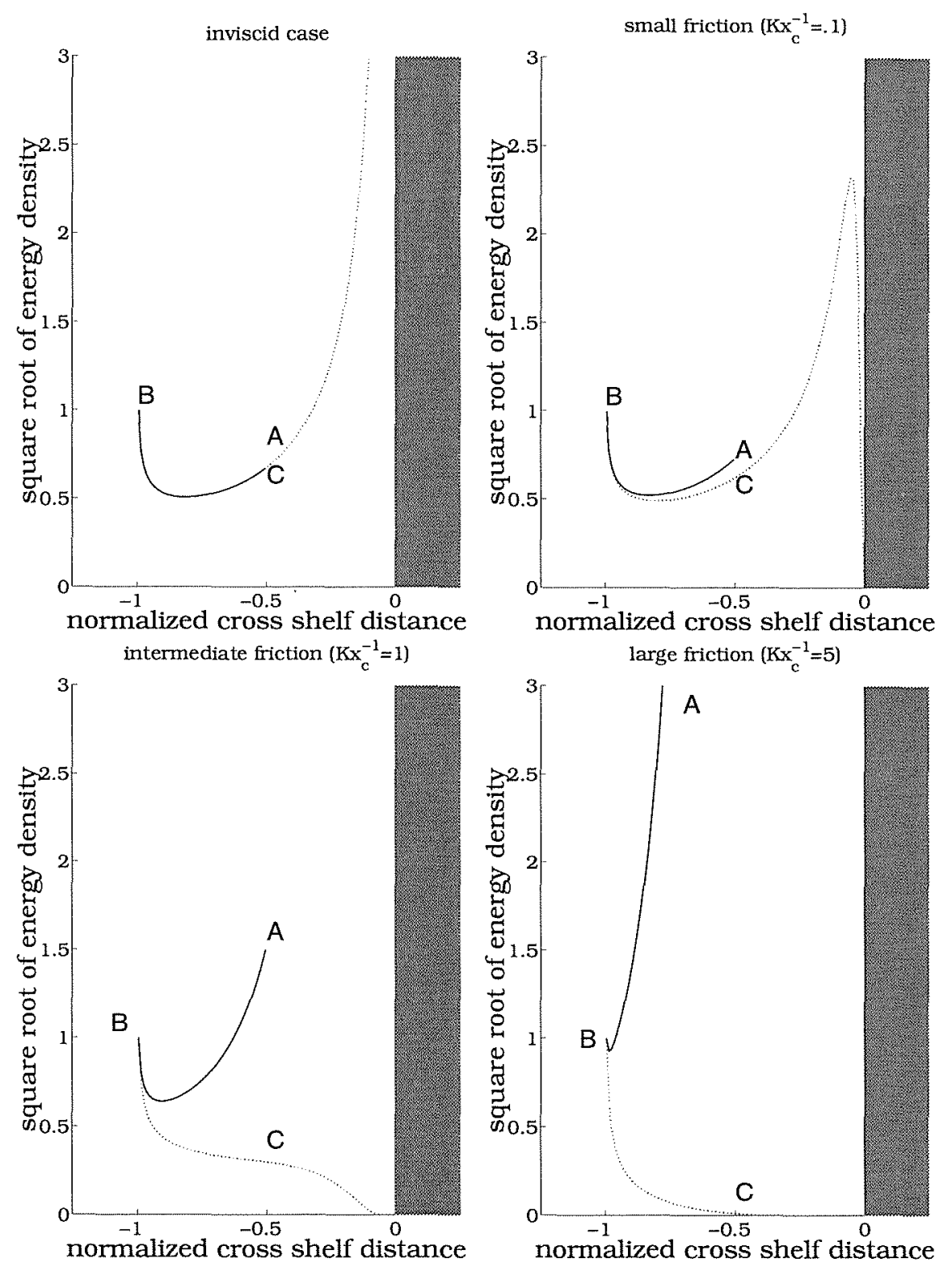

Figure 4.5: The evolution of the square root of the energy density $\left\langle E_{d}\right\rangle$ as a function of offshore distance for the internal wave whose path was traced in figure 4.4. The points marked $\mathrm{A}, \mathrm{B}$, and $\mathrm{C}$ are the points where the ray originated, was reflected back to shore, and passed by on the way to the coast. The solid line is the wave moving offshore, from points $\mathrm{A}$ to $\mathrm{B}$, and the dashed line the wave moving inshore, from points $\mathrm{B}$ to $\mathrm{C}$ to the coast. The top left plot is the inviscid case, the top right plot the nearly inviscid $x_{c} \gg K$ case, the bottom left plot where $x_{c} \approx K$, and the bottom right plot is the frictionally dominated $x_{c} \ll K$ case. 

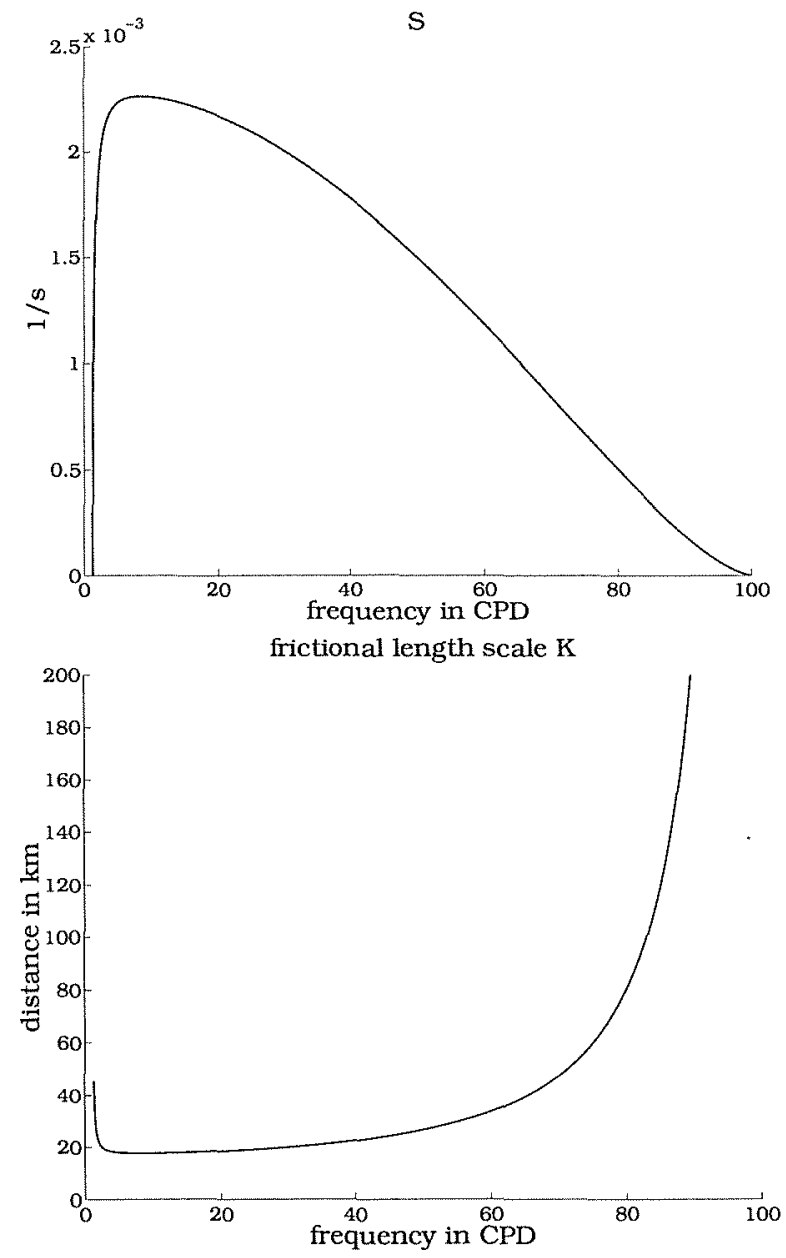

Figure 4.6: The top panel is a plot of $\mathrm{S}$, the quantity defined by (4.22) which, when multiplied by the depth, gives the group velocity of the wave for a mode one wave with $N=100 \mathrm{cpd}$ and $f=1.24 \mathrm{cpd}$. A mode two wave would have half the speed, a mode three wave a third the speed, etc. The bottom panel is the frictional length scale $K$ as a function of frequency. It is for a drag of $\mathrm{r}=5 \times 10^{-4} \mathrm{~m} \mathrm{~s}^{-1}$ and a bottom slope of $5 \times 10^{-3}$. All other parameters are as in the top plot. If the wave were mode 2 , the length scale would be twice as large because the group speed would be half as large. 


\subsection{Internal Waves in the Presence of a Barotropic Mean Flow}

Horizontal current shear causes an asymmetry in the propagation of internal waves over the shelf. To analyze this effect, the alongshore flow is idealized as a barotropic current $V(x)$ which varies in the cross shelf direction only. Olbers [1981] studied the problem of internal waves in a vertically sheared, geostrophic, and inviscid mean flow in an infinitely deep ocean, and his work illustrates the existence of critical layers in the vertical which are not possible in the present barotropic formulation.

In order to make the presentation clearer, $l_{0}$ will always be taken to be positive, which means the waves would always move in the direction of increasing $y$ in the absence of a mean flow. A mean current $V$ is then said to increase if it becomes more positive, and decrease if it becomes less positive. Only the cross-shelf shear of the mean velocity matters, for the spatial mean of the mean velocity can be removed by a change of reference frame. It is more natural to present the effects of a constant $V$ in the later sections describing what a current meter would measure, for the current meter does not translate alongshelf with the mean current.

The other major assumption, besides that of barotropic flow, is that the effective change in the local rate of rotation caused by the mean flow relative vorticity does not appreciably alter the dispersion relation for the intrinsic frequency of the wave, the frequency of the un-Doppler shifted wave given by (4.8). This amounts to an assumption that the intrinsic frequency is several times $f$, so that a change in $f$ does not affect the dispersion relation significantly, or that the mean flow has a low Rossby number. This criterion can be stated more formally by substituting the effective rotation rate of the flow from Kunze [1985], $\left(f^{2}+f V_{x}\right)^{\frac{1}{2}}$, into (4.8) and solving for the criterion for neglecting $V_{x}$. This criterion is

$$
\frac{1}{2} \frac{\partial V}{\partial x} \ll \sqrt{f^{2}+c^{2} N^{2}},
$$

and is easily met for realistic coastal flows and internal wave frequencies. Kunze [1985] studies the effect of trapping of near inertial waves by the variations in relative vorticity in a mean flow where this assumption is not met. It should also be remembered that if the wave's intrinsic frequency approaches $f$, the vertical mode assumption will break down as described by equation (4.6). 
Within these assumptions, the dispersion relation for modal internal waves can be written as

$$
\omega=\Gamma(x) l_{0}+\omega_{r}\left(k, l_{0}, M, D\right)
$$

where $\omega_{r}$ is the intrinsic frequency found from the dispersion relation for the unDoppler shifted wave (4.8). Since the mean flow does not vary in $y$ or time, $\omega, l_{0}$, and $M$ are, as before, conserved along a ray. Thus as the wave encounters shear in the mean flow, $\omega_{r}$ is constrained to vary between $N$, as $|k| \rightarrow \infty$, and $\omega_{\text {min }}$, as $|k| \rightarrow 0$, where $\omega_{\min }$ is

$$
\omega_{\text {min }}=\omega_{r}\left(k=0, l_{0}, M, D\right)=\sqrt{\frac{l_{0}^{2} N^{2}+f^{2} \frac{M^{2} \pi^{2}}{D^{2}}}{l_{0}^{2}+\frac{M^{2} \pi^{2}}{D^{2}}}} .
$$

Thus the wave can only exist when

$$
\omega_{\min } \leq \omega-V(x) l_{0} \leq N
$$

The group velocity is altered by the addition of the alongshore flow $V$ to the intrinsic group velocity:

$$
\overrightarrow{c_{g}}=\left(\hat{i} \frac{\partial}{\partial k}+\hat{j} \frac{\partial}{\partial l}\right) \omega=V(x) \hat{j}+\overrightarrow{c_{g r}}
$$

where $\overrightarrow{c_{g r}}$ is the group velocity of the wave in the absence of the flow, which is defined by (4.22).

The energy $E$ of the wave is not conserved in the presence of a mean sheared flow, for the mean flow can serve as an infinite source or sink or internal wave energy. However, Lighthill [1978] shows that the wave action

$$
E_{a}=\frac{E}{\omega_{r}}
$$

is conserved by a wave. Thus equation (4.29) becomes

$$
\frac{\partial}{\partial x}\left(D \frac{\left\langle E_{d}\right\rangle}{\omega_{r}}\left|\overrightarrow{c_{g r}}\right| \cos (\theta)\right)=-\frac{T^{-1} D\left\langle E_{d}\right\rangle}{\omega_{r}}
$$

where the time scale of dissipation $T$ is as before. The absence of $V(x)$ in $(4.42)$ is because, by definition, no component of the mean flow is in the cross shelf direction. It is not possible to get general solutions for realistic $V(x)$ profiles, because of the 
complex dependence of $\left|\overrightarrow{c_{g r}}\right|$ on $\omega_{r}$ and thus $V(x)$. It is easy, however, to solve (4.42) with standard numerical techniques. Before examining the full solutions, however, it is worth examining some of the limits of the equation.

If friction is negligible (which, as was shown earlier, is never true near the shore), then (4.42) becomes

$$
D \frac{\left\langle E_{d}\right\rangle}{\omega_{r}}\left|\overrightarrow{c_{g r}}\right| \cos (\theta)=\text { constant }
$$

For the moment, assume $\omega_{r}$ does not approach $\omega_{\min }$ or $N$ and neglect the change of depth. If a wave is entering a region of increasing currents, it can be seen from (4.37) that $\omega_{r}$ decreases. As $\omega_{r}$ decreases, $\overrightarrow{c_{g r}}$ increases unless $\omega_{r} \approx f$, as can be seen in figure 4.6. In order for $\omega_{r}$ to decrease, $k$ must decrease, thus $|\theta|$ increases and $\cos \theta$ decreases. Whether the change in $\left\langle E_{d}\right\rangle$ is dominated by the change in $\omega_{r}$ and $c_{g r}$, which tend to cause $\left\langle E_{d}\right\rangle$ to decrease, or the change in $\cos \theta$ which tends to increase $\left\langle E_{d}\right\rangle$, depends on the details of the ray.

As a wave moves into a region of decreasing currents, $\omega_{r}$ increases, $\overrightarrow{c_{g r}}$ decreases, $|\theta|$ decreases and $\cos \theta$ increases. As above, the change of $\left\langle E_{d}\right\rangle$ depends on the relative changes in the $\cos \theta$ term versus the changes in $\overrightarrow{c_{g r}}$ and $\omega_{r}$.

Friction introduces another effect. As $\omega_{r}$ increases, the friction length $K$ increases (see figure 4.6). As $K$ increases, the wave is dissipated more rapidly.

The effects of a mean current on a ray will be small if the current does not alter the intrinsic frequency $\omega_{r}$ greatly. This is true when

$$
\frac{V l_{0}}{\omega} \ll 1
$$

Solving for $l_{0}$ from the dispersion relation for un-Doppler shifted waves and assuming that $f^{2} \ll \omega^{2} \ll N^{2}$, this criterion is met if

$$
V \ll \frac{N \alpha x_{c}}{M \pi}
$$

which for $\mathrm{N}=100 \mathrm{cpd}$ and $x_{c}=30 \mathrm{~km}$, means $V \ll 30 \mathrm{~cm} \mathrm{~s}^{-1}$, and for an $x_{c}$ of $60 \mathrm{~km}$, $V \ll 60 \mathrm{~cm} \mathrm{~s}^{-1}$.

If $V$ increases to the point that $\omega_{r} \rightarrow \omega_{\min }$ then $k \rightarrow 0$, which produces a caustic of the same form as described earlier in the $x \rightarrow x_{c}$ case. As in the $x \rightarrow x_{c}$ caustic, the wave energy is reflected from whence it came. The infinite amplitude suggested by (4.43) as $\left|\overrightarrow{c_{g r}}\right|$ goes to zero does not occur, and instead there is only a weak local maximum in $\left\langle E_{d}\right\rangle$ as in the $x \rightarrow x_{c}$ caustic. 
If $V$ decreases to the point that $\omega_{r} \rightarrow N$ then $k \rightarrow \pm \infty$. Because $\omega_{r} \rightarrow N$, $\left|\overrightarrow{c_{g r}}\right| \rightarrow 0$, as illustrated in figure 4.6. The ray tracing theory does not break down, since as $k \rightarrow \pm \infty$ the horizontal scale of the wave becomes less. The ray does not get reflected back but instead asymptotically approaches the location where $\omega_{r}=N$. As in the case where the ray goes to the shore, friction dominates the cross shelf evolution of $\left\langle E_{d}\right\rangle$ as $\left|\overrightarrow{c_{g r}}\right| \rightarrow 0$, causing the amplitude to go eventually to zero instead of the infinity predicted by the inviscid equations. The power in the horizontal currents does not increase as much as $\left\langle E_{d}\right\rangle$ because the increase is offset by the change of proportionality constant between horizontal power and $\left\langle E_{d}\right\rangle$ with $\omega_{r}$. The local increase in amplitude, and the subsequent decrease in amplitude as friction begins to dominate, are illustrated in figure 4.7. This figure follows the square root of $\left\langle E_{d}\right\rangle$ along a ray launched near its $x_{c}$, where $V=0$, into a region where $V$ decreases. The steady decrease of the group velocity as the wave approaches $10 \mathrm{~km}$ offshore can be seen in the slope of the position versus time plot.

The velocity needed to force $\omega_{r} \rightarrow N$ depends on $\omega$ and $l_{0}$, while the velocity needed to force $\omega_{r} \rightarrow \omega_{\min }$ depends on $\omega, l_{0}, M$ and the depth. The velocities needed to force $\omega_{r} \rightarrow N$ have been plotted in figure 4.8 as a function of $\theta$ for a wave at the $200 \mathrm{~m}$ isobath, where $V$ is presumed to be zero. Since the velocity needed to force $\omega_{r} \rightarrow \omega_{\min }$ depends on the water depth, figure 4.9 plots this velocity for a wave at a depth of 100 meters as a function of the onshore angle $\theta$ of the wave at the $200 \mathrm{~m}$ isobath, where $V$ is again presumed to be zero.

The asymmetry with respect to the alongshore direction of the response to $\omega_{r} \rightarrow N$ and $\omega_{r} \rightarrow \omega_{m i n}$ raises the possibility of an alongshore internal waveguide for waves with alongshore intrinsic group velocity oriented so that $V(x)$ increases toward the coast. If the shear in $V(x)$ is strong enough, the wave will move inshore until $\omega_{r} \rightarrow \omega_{\min }$. It will then be reflected back to deeper water. If it then reaches $x_{c}$ before $\omega_{r} \rightarrow N$, it will be reflected back toward shore, reflecting between the two caustics until it has dissipated. A wave whose alongshore intrinsic group velocity is in the other direction will experience a $V(x)$ that decreases in the onshore direction, and will thus be dissipated at the coast or where $\omega_{r} \rightarrow N$. 

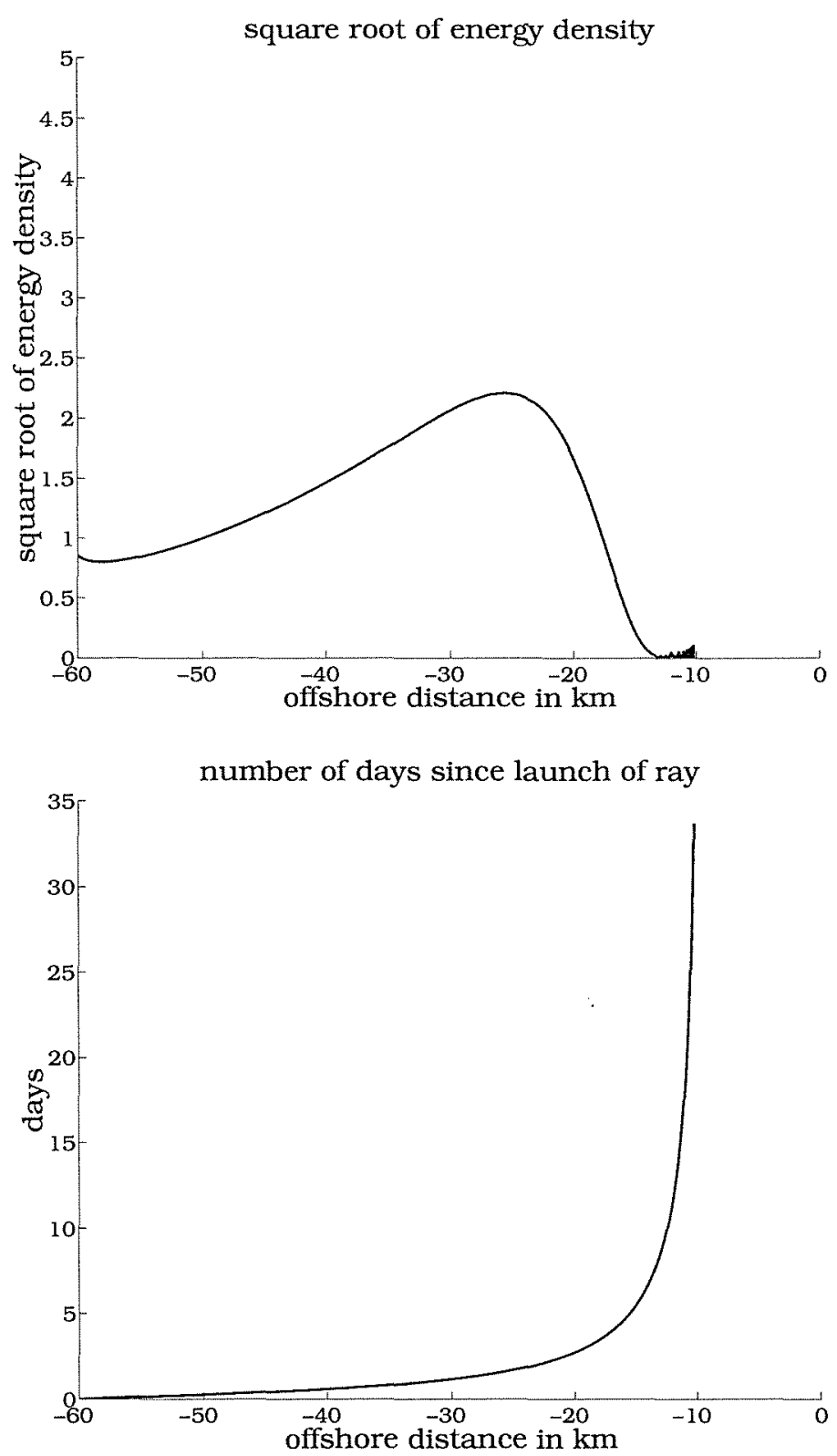

Figure 4.7: The top panel is a plot of the square root of $\left\langle E_{d}\right\rangle$ for a wave launched from $60 \mathrm{~km}$ offshore into a region where the mean currents are becoming more negative. The point where $\omega_{r}=N$ is $10 \mathrm{~km}$ offshore. The bottom panel is a plot of the number of days since launch that it has taken the ray to get to a point. $\mathrm{N}=100, f=1.24$. $\omega$ is $20 \mathrm{cpd}$ and current goes linearly from 0 at $60 \mathrm{~km}$ offshore to $-2.8 \mathrm{~m} \mathrm{~s}^{-1} 15 \mathrm{~km}$ offshore. 


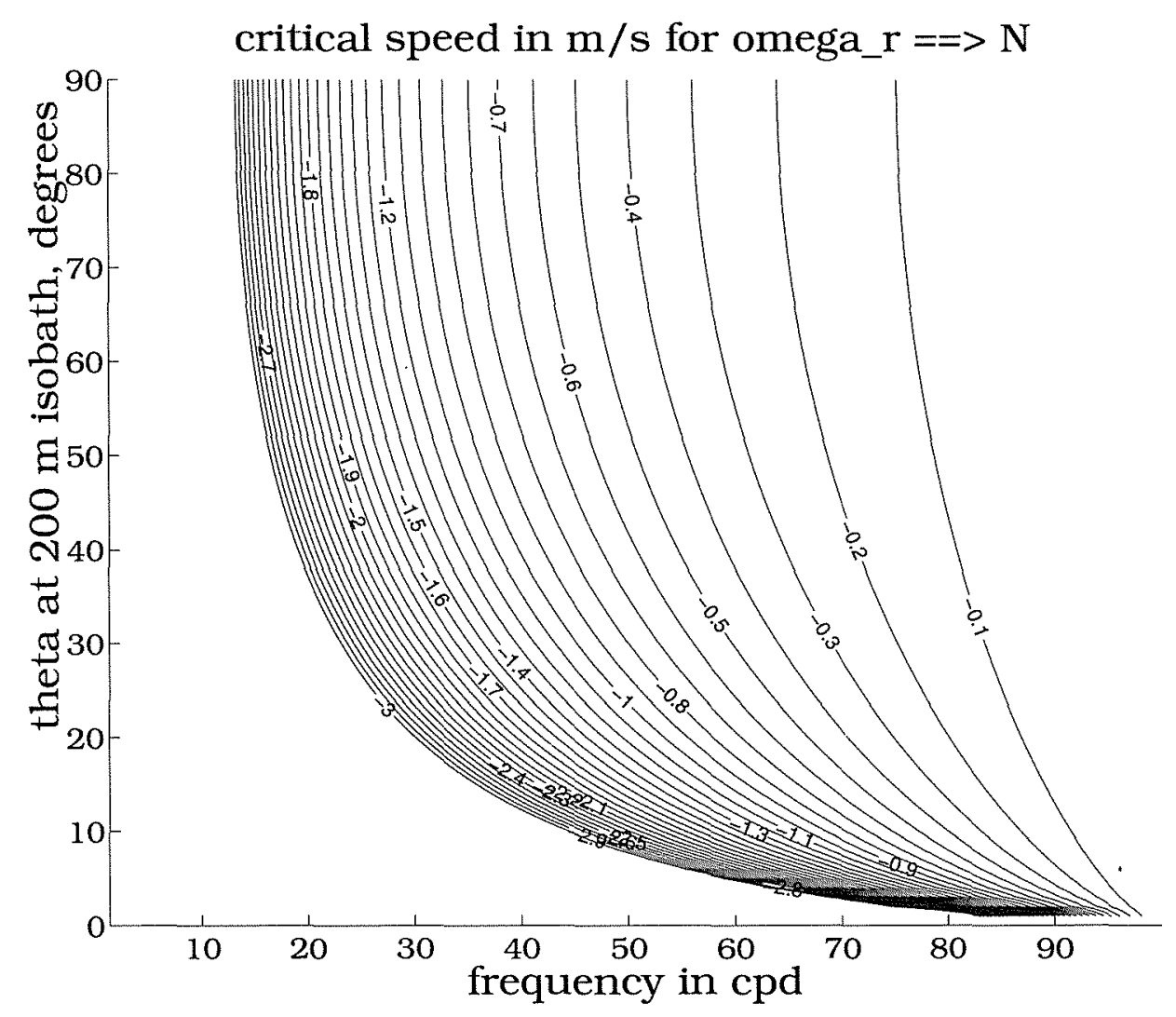

Figure 4.8: The contours of this figure are the velocities in $\mathrm{m} \mathrm{s}^{-1}$ needed to make $\omega_{r} \rightarrow N$ for a wave with a given frequency and whose angle to the coast at the 200 meter isobath was $\theta$. The water is assumed to be quiescent at the 200 meter isobath. 


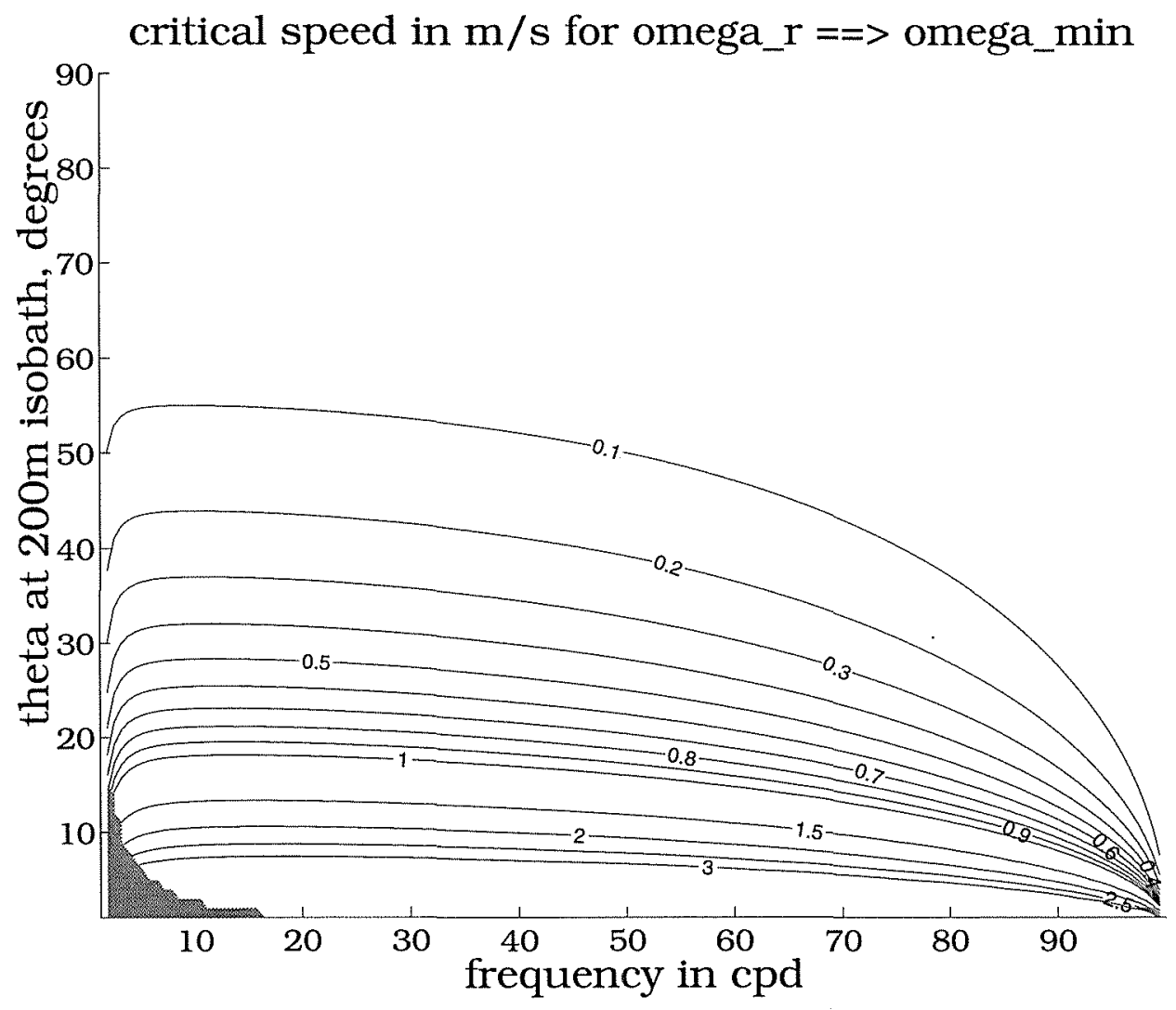

Figure 4.9: This is a plot of the velocity needed to make $\omega_{r} \rightarrow \omega_{m i n}$ at 100 meters depth in terms of the frequency and the angle the wave had to the cross shore direction at the 200 meter isobath, where $V$ is assumed to be zero. The shaded region in the lower left hand corner is where $\omega_{\text {min }}$ is close enough to $f$ that the vertical mode assumption breaks down for a bottom slope of $5 \times 10^{-3}$ 


\subsection{Current Meter Observations- The No Mean Flow Case}

The preceding sections have discussed the evolution of a single ray as it travels on- or offshore. Since most observational techniques only measure currents or temperatures at a point, it is necessary to integrate over all the rays that go through a point to predict the currents at that point. This section illustrates how a very simple model of internal waves on the shelf can predict the observations at a current meter mooring. Vertical current resolution at a point is assumed to be dense enough to resolve the vertical modes. The observables discussed are the total power at a frequency and the ellipticity of the current ellipse at that frequency (The ellipticity is the square of the ratio of the major and minor axes of the current ellipse).

The model assumes that all waves propagating onshore have the same amplitude, regardless of orientation. This assumption may not be valid in general, but, lacking a better knowledge of the internal wave climate at the shelf break, it is a useful first assumption. This spectrum begins to "feel" the bottom at a distance offshore of $x_{b}$. As discussed above, $x_{b}$ could be either where the line of $N=\omega$ intersects the bottom, where the bottom slope stops exceeding the characteristic slope of internal waves, $c$, or where the bottom becomes flat. The mean alongshore current is taken to be zero in this section, but this assumption is relaxed in the next section. The bottom inshore of $x_{b}$ has constant slope $\alpha$ as before.

By assumption the rays at $x_{b}$ all have the same amplitude for a given frequency, and are distributed over the angles $-90^{\circ} \leq \theta \leq 90^{\circ}$ where $\theta$ is the angle of the ray at $x_{b}$. To model this, the constant $\mathcal{C}$ in (4.32) has been set so that each ray has amplitude $\pi^{-1}$ at $x_{b}$. Thus (4.32), which is the energy density along a ray, becomes

$$
\left\langle E_{d}\right\rangle=\frac{1}{\pi} \frac{x_{b}^{2} \cos (\theta)}{x^{2} \sqrt{1-\frac{x^{2}}{x_{b}^{2}} \sin ^{2}(\theta)}} \exp \left[\left(\frac{\sqrt{1-\frac{x^{2}}{x_{b}^{2}} \sin ^{2}(\theta)}}{x}-\frac{\cos (\theta)}{x_{b}}\right) \frac{2 r}{S \alpha^{2}}\right] .
$$

In order to convert this into the power observed at a fixed site, it is assumed the rays have random phase. Then the observed power in a mode, $\overline{\left\langle E_{d}\right\rangle}$, is just the integral of (4.46) over $-90^{\circ} \leq \theta \leq 90^{\circ}$. This integral is simple to evaluate numerically.

To calculate ellipticity, however, it is necessary to rotate the wave velocity components in (4.2) into the angle of the ray at the current meter, $\theta_{m}$. Once this 
is done, the power in the cross shelf direction, $P_{u u}$, and the power in the alongshore direction, $P_{v v}$, can be calculated as

$$
\begin{aligned}
P_{u u} & =\left\langle E_{d}\right\rangle\left(\cos ^{2}\left(\theta_{m}\right)+\frac{f^{2}}{\omega^{2}} \sin ^{2}\left(\theta_{m}\right)\right), \\
\text { and } \quad P_{v v} & =\left\langle E_{d}\right\rangle\left(\sin ^{2}\left(\theta_{m}\right)+\frac{f^{2}}{\omega^{2}} \cos ^{2}\left(\theta_{m}\right)\right),
\end{aligned}
$$

where $\theta_{m}$ can be calculated from (4.11) and the definition of the depth $D=-\alpha x$ :

$$
\theta_{m}=\arctan \left(\frac{\sin (\theta)}{\sqrt{\frac{x_{b}^{2}}{x^{2}}-\sin ^{2}(\theta)}}\right) .
$$

The expressions for $P_{u u}$ and $P_{v v}$ must be integrated over all $\theta$ to account for all rays the current meters observe, which leads to an ellipticity of

$$
\text { ellipticity }=\frac{\int_{-90^{\circ}}^{90^{\circ}} P_{u u} d \theta}{\int_{-90^{\circ}}^{90^{\circ}} P_{v v} d \theta}
$$

As with the power, the integrals are easily solved numerically.

The limits of ellipticity and power as $x$ limits to $x_{b}$ and the coast are visible by inspection. As $x$ approaches $x_{b},\left\langle E_{d}\right\rangle$ of a ray goes to $\pi^{-1}$, because that is the initial condition. The local angle $\theta_{m}$ will limit to $\theta$ at the same time. Thus the energy density observed by the current meter $\overline{\left\langle E_{d}\right\rangle}$, which is the integral of $\left\langle E_{d}\right\rangle$ over $\theta$, will approach one as $x$ goes to $x_{b}$. The ellipticity will also go to one as $x$ goes to $x_{b}$. This recovers the initial condition of an isotropic wave field with an $\overline{\left\langle E_{d}\right\rangle}$ of one.

It was shown in section (4.5) that with even infinitesimal bottom friction, as $x$ goes to zero $\left\langle E_{d}\right\rangle$ on each ray goes to zero, and thus the power integrated over all rays goes to zero at the coast. The ellipticity, however, is the ratio of two powers and does not go to zero. As $x$ goes to zero, $\theta_{m}$ approaches $\theta x / x_{b}$. Thus $\cos \left(\theta_{m}\right)$ limits to one and $\sin \left(\theta_{m}\right)$ limits to $\theta x / x_{b}$ as $x \rightarrow 0$. (4.49) then limits to $\omega^{2} / f^{2}$. This is intuitively acceptable because as the waves approaches the shore, they becomes more tightly focused in the cross shore direction, until all of the rays are headed very nearly inshore. Thus the ellipticity asymptotes to the plane wave limit of $\omega^{2} / f^{2}$.

The strength of friction does not affect the ellipticity significantly if the only internal wave signal on the shelf is that of the internal waves arriving from offshore. As seen in figure 4.10 a factor of 16 increase in the ratio of $K$ to $x_{b}$, from 0.25 to 4 , 
greatly increases the attenuation of power going onshore but only marginally affects the ellipticity of the wave. This is because the waves are very quickly turned towards the shore by the bathymetry, and the subsequent variation in the path length between different waves is minimal. Thus friction attenuates all waves to a similar extent.

Perhaps more surprisingly, the ellipticity is not strongly dependent on frequency until $x / x_{b}$ becomes small, as can be seen in figure 4.11. Also plotted in this figure is the ellipticity of a group of waves whose individual ellipticities are infinite (this is the $f=0$ case). The independence of the ellipticity from $\omega$ holds when the ellipticity predicted for the $f=0$ case is less than $\omega^{2} / f^{2}$, the ellipticity of a single wave. In that limit, the ellipticity is dominated by the angular distribution of rays, not the ellipticity of the currents of each individual ray.

Friction does, however, play a strong role in the observed power. The higher the friction, the lower the power as one moves inshore. The plots of $\overline{\left\langle E_{d}\right\rangle}$ versus cross shelf distance in figure 4.10 have the same qualitative dependence on the ratio of $K / x_{b}$ as the individual rays had to the ratio of $K / x_{c}$ in previous sections. Since the friction length scale increases with frequency and mode (see figure 4.6), higher frequencies and higher modes are more attenuated as they move onshore. Thus, whatever the frequency spectrum at $x_{b}$, it should redden as one moves inshore, and become more dominated by low mode waves.

It is unrealistic to expect that the only internal waves on a shelf are the ones propagating from offshore. To represent the presence of waves generated on the shelf, the model has been modified to include an isotropic background noise whose amplitude is everywhere $10 \%$ of the energy density at $x_{b}$. This isotropic wave field is meant to be a representation of the waves generated on the shelf itself. It must be a poor model, if for no other reason than that the wave field generated on the shelf is unlikely to be isotropic. In the absence of some knowledge of the mechanism for the generation of internal waves on the shelf, it is unclear what would be more sensible, however. While the total internal wave energy is only slightly altered by the addition of the isotropic wave field, the observed ellipticity near the coast is dominated by the isotropic wave field, since the energy of the waves propagating from $x_{b}$ has been attenuated by the passage across the shelf. This is clearly seen in figure 4.12 , in which the ellipticity and $\left\langle E_{d}\right\rangle$ are plotted for the same three ratios of $K$ to $x_{b}$ as in figure 4.10 , but with the added isotropic wave field. All of the ellipticities now approach one near the coast because the signal is dominated by the isotropic wave field near the 

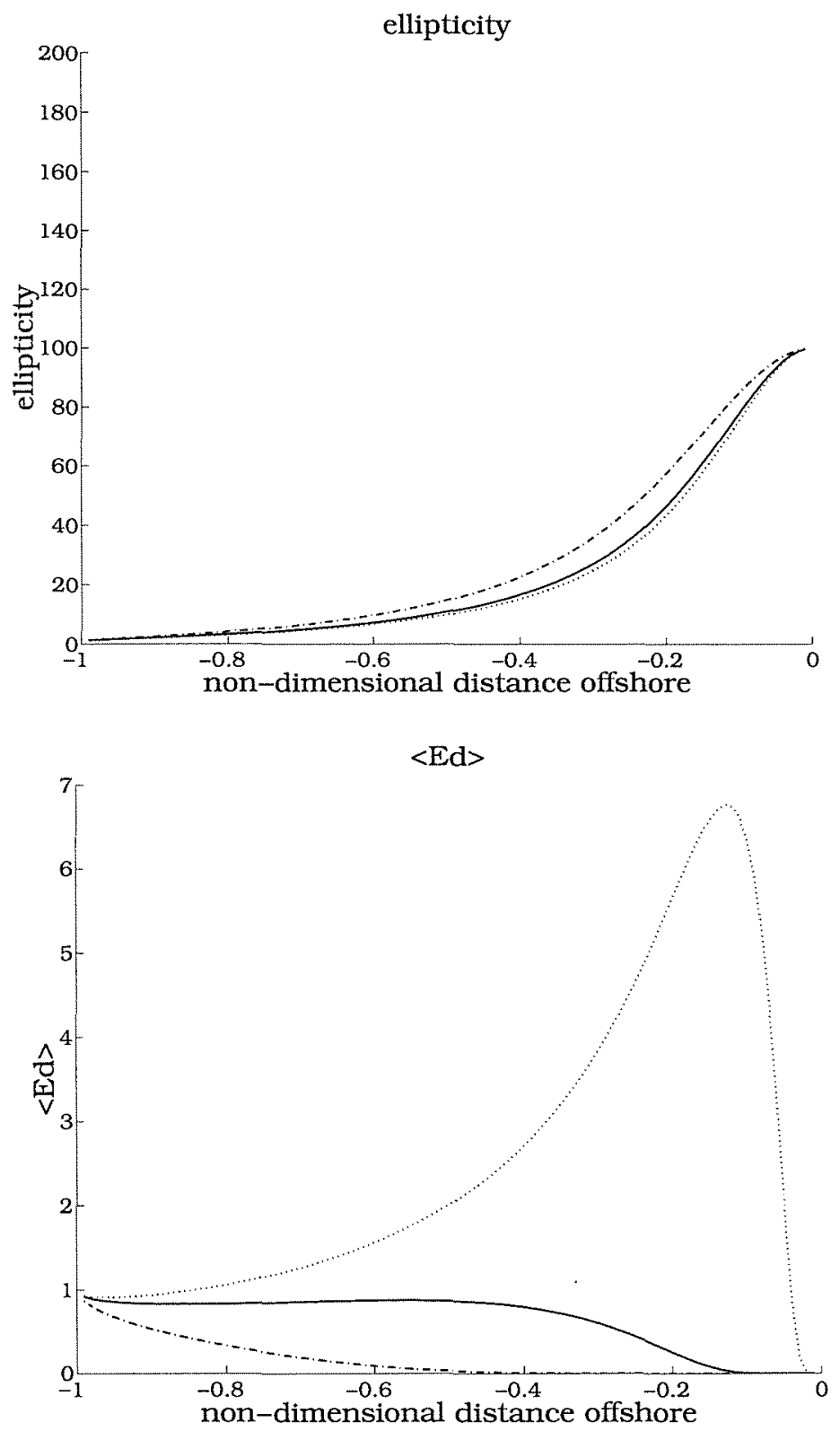

Figure 4.10: The top panel is a plot of the ellipticity observed by current meters for a frequency of $10 f$ and for three different values of the ratio $K / x_{b}$, the dotted line for 0.25 , or weak friction, the solid line for 1 , and the dashed line for 4 , or strong friction. The bottom panel is a plot of the energy density observed by a current meter for the same three ratios of $K / x_{b}$. The cross shore distance has been normalized by $x_{b}$ for both plots. 


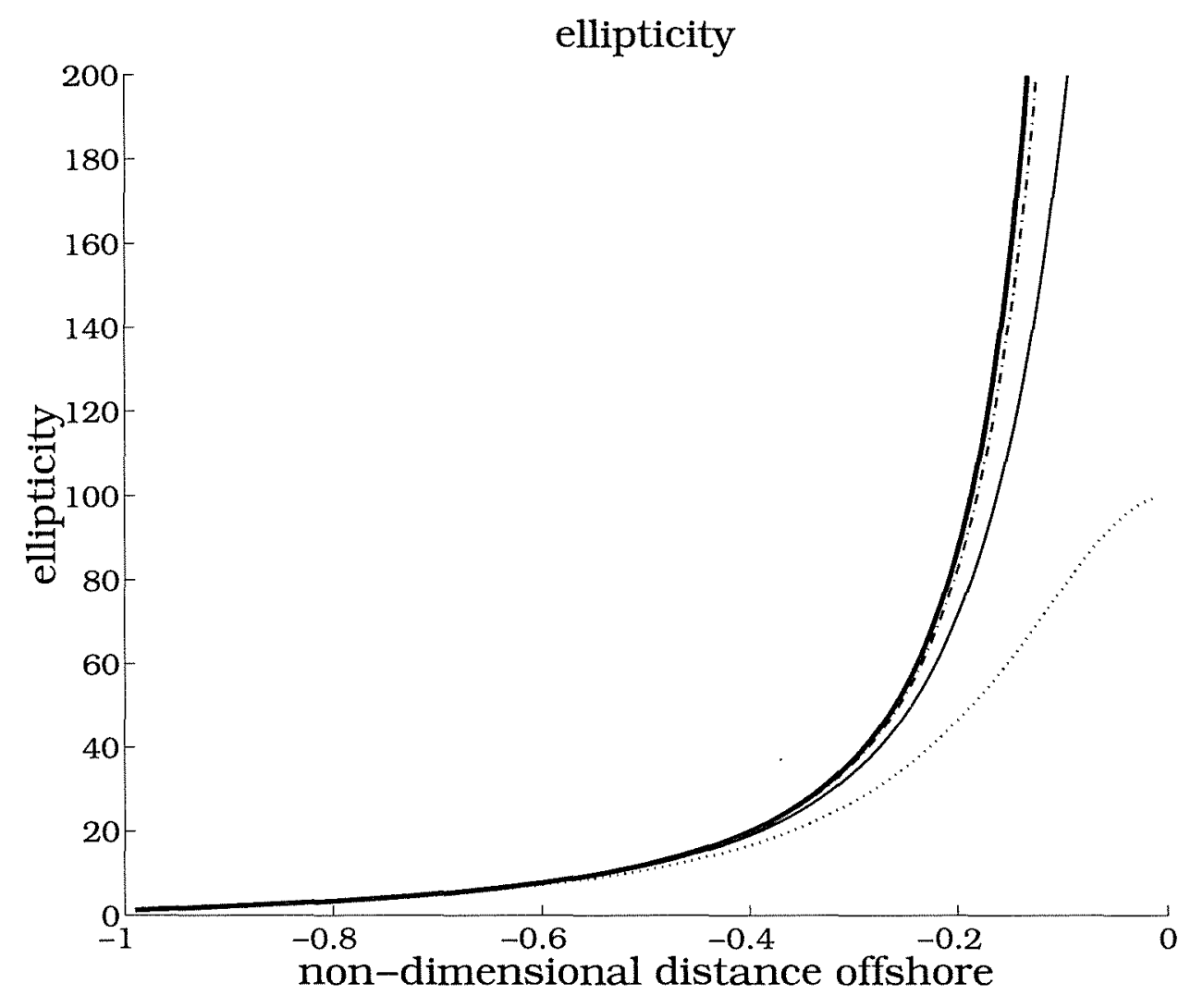

Figure 4.11: The ellipticity of the current ellipse observed at frequencies of 10, 20 and 40 times $f$ as a function of the cross shore distance normalized by $x_{b}$. The dotted, solid, and dashed lines correspond to $\omega$ equaling $10 \mathrm{f}, 20 \mathrm{f}$ and $40 \mathrm{f}$. $K=x_{b}$. The heavy line is the ellipticity observed for an ensemble of waves each of whose ellipticity is infinite. This would be the case if $f=0$. 
coast, and the greater the friction, the faster the waves from offshore are attenuated and the more the isotropic wave field dominates the ellipticity. The lesson in this is not in the exact shape of the curves in figure 4.12 , since the model of waves generated on the shelf is naive. The lesson is that the broader a shelf is, or the stronger friction is, the less the propagation of waves from the deep ocean matters and the more the generation of waves on the shelf matters to the wave climate on the shelf. Because friction is more effective for higher frequency and higher vertical modes, observations of higher frequency and higher mode waves will be more affected by the generation of waves on the shelf.

\subsection{Current Meter Observations- The Mean Flow \\ Case}

The model described above can be extended to include mean currents, so that the anisotropy introduced by the mean currents can be studied. However, the interpretation of the results becomes more difficult. The problem lies not only in the prediction of the evolution of any given ray, but the identification of which rays will have a frequency $\omega$ at the current meter, and what the initial amplitude of those rays was. Because of this a $V$ which does not vary in the cross shelf direction will have important effects that were not present in the analysis of single rays in the presence of mean flows. To make the extension of the model simpler, the shears are chosen to be small enough that $\omega_{r}$ does not go to $\omega_{\text {min }}$, so no waves are reflected back to the shelf break. This assumption places constraints on the alongshore currents that can be surmised from figure 4.9 and equation 4.38 .

The initial conditions of the rays at $x_{b}$ are now modeled as a Garrett and Munk (GM) spectrum even though the GM spectrum is not meant to be applicable near vertical or horizontal boundaries [Garrett and Munk, 1972]. The amplitude of the internal waves at $x_{b}$ is taken to depend on $\omega_{r}^{-2}$. This means the spectrum is isotropic at $x_{b}$ in the moving reference frame defined by $V\left(x_{b}\right)$, or, equivalently, the reference frame in which $V\left(x_{b}\right)=0$ is special for the whole shelf. In any other reference frame, the internal wave field at $x_{b}$ is anisotropic, for waves at a given $\omega$ which are Doppler shifted from lower $\omega_{r}$ will have greater amplitudes than those Doppler shifted from higher $\omega_{r}$. Thus waves traveling with the current ( $V$ and $l_{0}$ of the same sign) will 

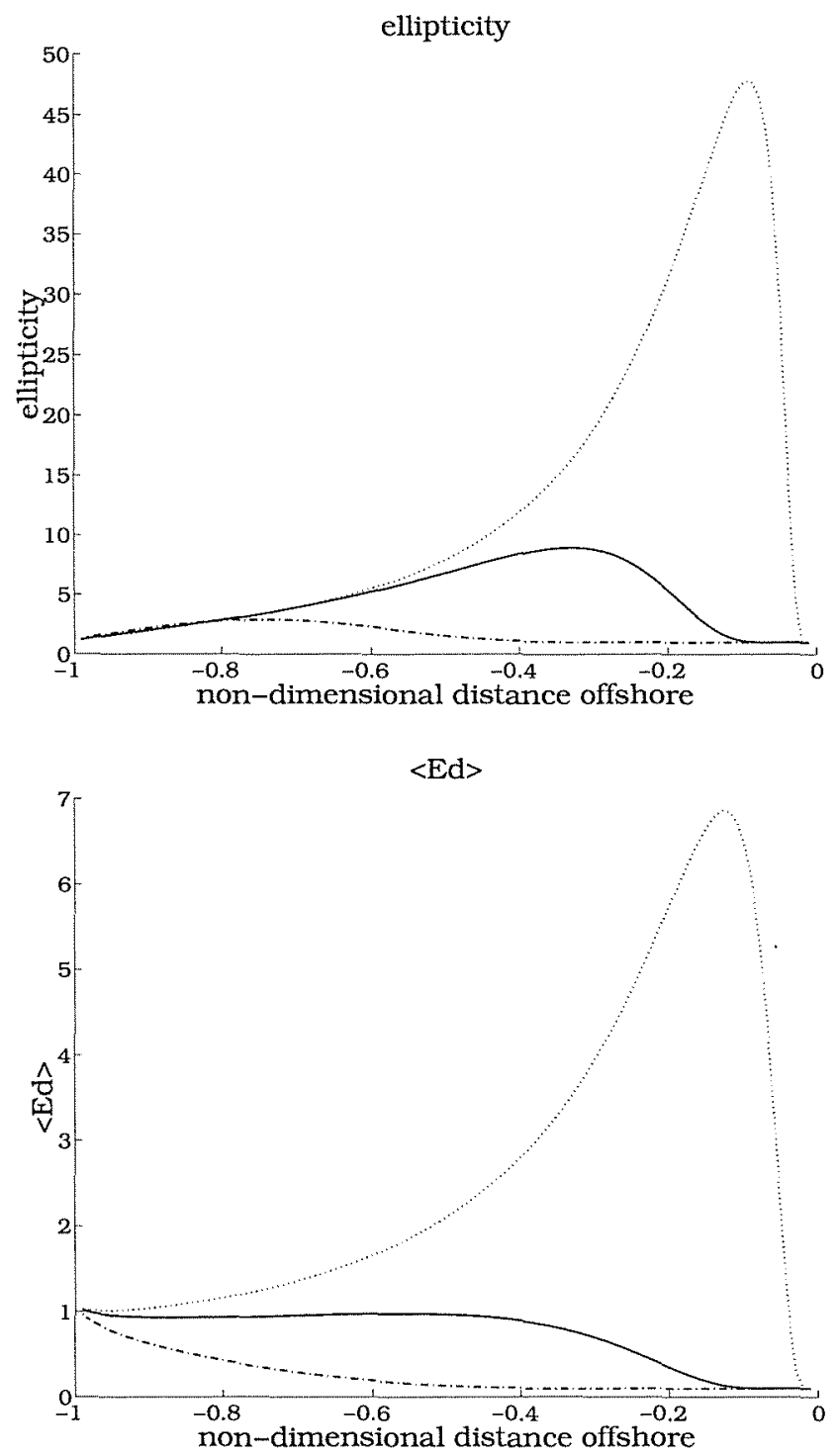

Figure 4.12: The same plot as figure 4.10 except an isotropic noise field whose $\overline{\left\langle E_{d}\right\rangle}$ is ten percent of the $\overline{\left\langle E_{d}\right\rangle}$ at $x_{b}$ has been added. The top panel is a plot of the ellipticity observed by current meters for a frequency of $10 \mathrm{f}$ and for three different values of the ratio $K / x_{b}$, the dotted line for 0.25 , the solid line for 1 , and the dashed line for 4. The bottom panel is a plot of the energy density observed by a current meter for the same three ratios of $K / x_{b}$. The cross shore distance has been normalized by $x_{b}$ for both plots. Notice that the ellipticities are so greatly reduced by the addition of the noise that the scale on the top panel is different from figure 4.10. 
be observed by a fixed observer at $x_{b}$ to have greater amplitude than those moving against the current. Whether this is a correct initial condition depends on what sets the GM spectra, and how quickly it equilibrates, questions not addressed here.

The solution for these initial conditions involves a straightforward substitution of equation (4.42) for (4.31) in the previous model with two modifications. The first is that a current meter observes not the energy density of a wave, but the power in the horizontal currents. Thus (4.47) must be multiplied by the conversion from $\left\langle E_{d}\right\rangle$ to to horizontal current power given in equation (4.18) before (4.47) is integrated over all incoming rays. This conversion factor is a function of $\omega_{r}$.

The second complication is that for a given angle $\theta$ of the horizontal wave vector to the onshore direction at $x_{b}$, there can be more than one ray with frequency $\omega$. In order to find all the rays with a frequency $\omega$ and an angle $\theta$, the dispersion relation (4.37) is written in terms of $\theta$ and the magnitude of the horizontal wave vector $|\vec{k}|$ :

$$
\pm \omega=|\vec{k}| \sin (\theta)+\sqrt{\frac{|\vec{k}|^{2} N^{2}+\frac{M^{2} \pi^{2}}{D^{2}} f^{2}}{|\vec{k}|^{2}+\frac{M^{2} \pi^{2}}{D^{2}}}}
$$

The \pm in front of $\omega$ is necessary to include all waves that a current meter would observe with a frequency $\omega . \omega_{r}$ must always be positive, as it is defined to be in (4.8), (4.37) and (4.50), so that the wavenumber vector $\vec{k}$ unambiguously indicates the direction of propagation of an un-Doppler shifted wave, but this does not constrain the sign of $\omega$. The model first solves for $|\vec{k}|$ for an evenly spaced subset of all $\theta$ onshore, and then for each $\theta$ traces the resulting rays toward the coast using (4.42). The total power and covariance are summed over all the possible waves. This is like the numerical integration over $\theta$ in the last section, but includes the possibility of multiple rays at a given $\theta$.

The anisotropy introduced by the addition of a mean current is quantified by examining the angle of the principal axis of the current ellipse to the cross shore direction. This is derived from the energy density of each ray, again assuming that the waves have random phase. Following Godin [1988] the angle of the major axis is found from

$$
\theta_{e l i p_{m}}=\frac{1}{2} \arctan \left(\frac{2 C o_{u v}}{P_{u u}-P_{v v}}\right)
$$


where $P_{u u}$ and $P_{v v}$ are the cross and alongshore powers as before, and $C o_{u v}$ is the cospectrum of the alongshore and cross shore velocities.

One source of anisotropy is the anisotropy in the initial amplitude of the rays at $x_{b}$ caused by the dependence of $\omega_{r}$ on the orientation of the ray when $V\left(x_{b}\right) \neq 0$. This will tend to tilt $\theta_{\text {elipm }}$ downstream since waves moving with the current will have been Doppler shifted from a lower $\omega_{r}$ and thus have greater amplitude.

However, even when $V\left(x_{b}\right)=0$ and the rays have the same amplitude when they leave $x_{b}$, the shear can alter the horizontal currents observed at a current meter. Not only can a mean sheared flow alter the energy density via the mechanisms outlined in section 4.6 , but as $\omega_{r}$ increases, a given $\left\langle E_{d}\right\rangle$ produces less horizontal current power (equation 4.18).

To describe the current meter observations in the presence of a mean shear flow, two mean current profiles will be examined. The first is a constant alongshore flow $V_{0}$. Since $V$ is the same everywhere, $\omega_{r}$ is constant along a ray, and since $\omega_{r}$ is constant along a ray, only the effect of the anisotropy of the initial condition at $x_{b}$, and the different $K$ for each ray, affects $\theta_{\text {elipm }}$. The other current profile varies from 0 at $x_{b}$ linearly to $V_{0}$ at the shore. Since $\omega_{r}=\omega$ at $x_{b}$, all the rays start with the same amplitude and $\theta_{\text {elip }}$ is only affected by the change in $\omega_{r}$ along the ray.

The actual parameters used in the model runs are meant to resemble those for the CODE region on the northern Californian shelf. Data from this region are examined in chapter 5 . The bottom friction is $5 \times 10^{-4} \mathrm{~m} \mathrm{~s}^{-1}$, the bottom slope is $5 \times 10^{-3}, x_{b}$ is $30 \mathrm{~km}, f$ is $1.24 \mathrm{cpd}$, and $N$ is $100 \mathrm{cpd}$. The magnitude of the current $V_{0}$ is chosen as $0.1 \mathrm{~m} \mathrm{~s}^{-1}$, and each current profile is studied with $\omega$ equal to 10 and $40 \mathrm{cpd}$. These runs were repeated with different values of $r$, and the ellipticity and the orientation of the current ellipses were found to be insensitive to friction.

The first current profile, $10 \mathrm{~cm} \mathrm{~s}^{-1}$ everywhere, does not have a symmetric distribution of energy around $\theta_{0}=0$ because the intrinsic frequency $\omega_{r}$, and hence the initial power in each ray, are asymmetric around $\theta=0$. All rays oriented downstream $\left(\theta_{0}>0\right)$ have $\omega_{r}<\omega$ and all upstream rays have $\omega_{r}>\omega$, as can be seen in figures 4.13 and 4.14. For every $\theta<0$, there are three possible $\omega_{r}$, though two are near $N$ and so contribute less than $5 \%$ of the observed currents at a given location. These rays whose $\omega_{r}$ is near $N$ have small group velocities, and thus their ray paths are obliquely incident to the coast in figures 4.13 and 4.14. The angle of the major axis to the shore moves to zero as observations are made closer to shore, again as illustrated in 

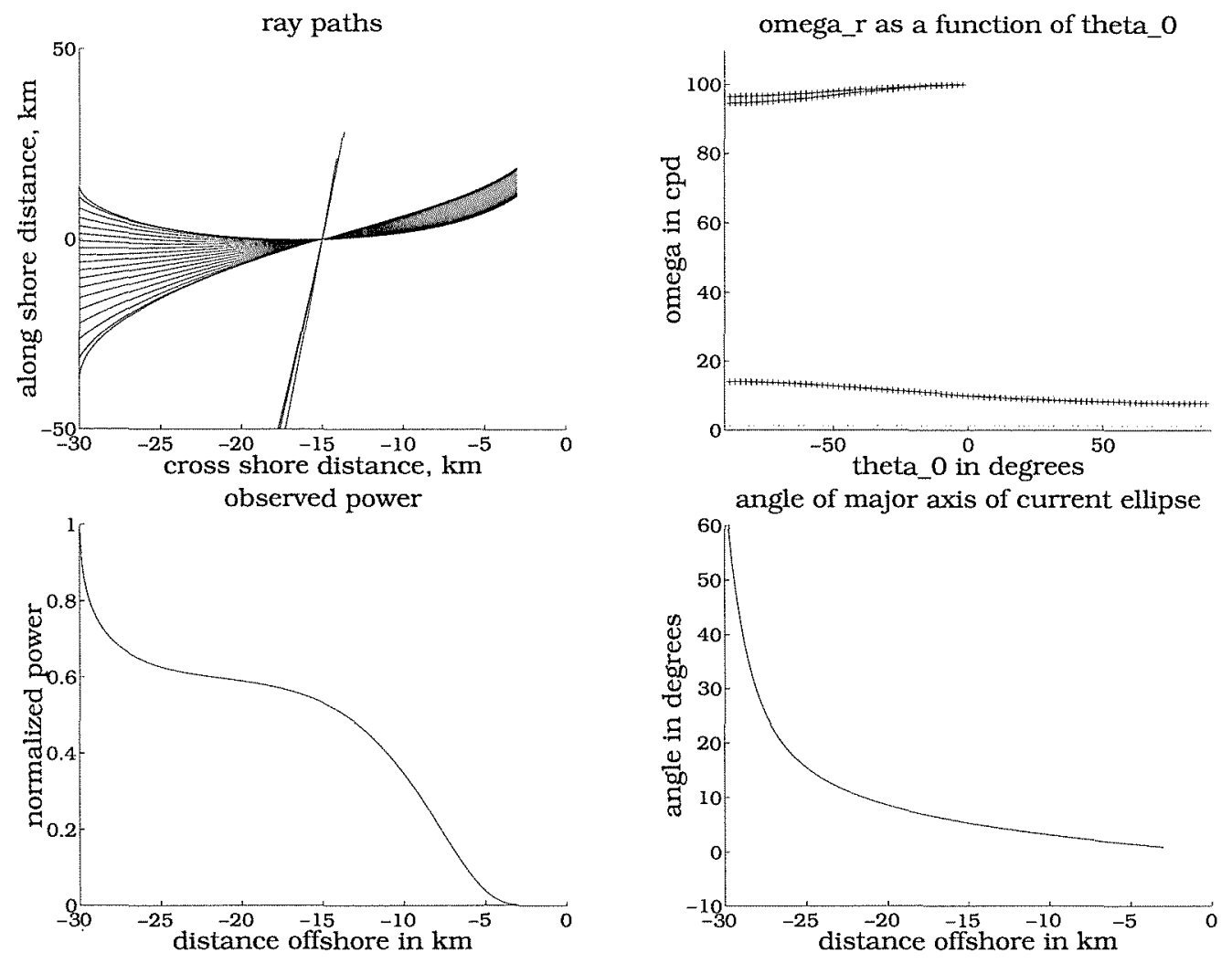

Figure 4.13: This is a plot of the current meter observation model for the $\omega=10 \mathrm{cpd}$, $V=10 \mathrm{~cm} \mathrm{~s}^{-1}$ case. The top left panel shows the ray paths for all rays which reach 15 $\mathrm{km}$ offshore within 30 days. The top right panel shows the initial $\omega_{r}$ of the rays as a function of $\theta$ at $x_{b}$. The dotted horizontal lines are $f, \omega$ and $N$. The bottom left panel is the evolution of the horizontal current power observed by a current meter as a function of cross shelf distance, while the bottom left panel is the angle that the major axis of the current ellipse makes with the cross shelf direction.

figures 4.13 and 4.14. This is because as the rays approach the shore, the angle they make to the cross shelf direction goes to zero, and thus the angle of the major axis of the current ellipse approaches zero. This occurs rapidly, with the angle becoming less than $10^{\circ}$ for the $\omega=10$ cpd case when the wave has only moved one quarter of the way toward the shore. Doubling or halving the bottom friction changes the major axis angle less than 10\%, though of course as friction increases the solution would become more sensitive to the generation of internal waves over the shelf.

There are no qualitative changes between the $\omega=10$ and 40 cpd cases, though the power decays faster in the cross shelf direction and $\theta_{\text {elip }}$ is greater in the $40 \mathrm{cpd}$ case. The faster decay in power comes about because of the greater $K$ for higher 

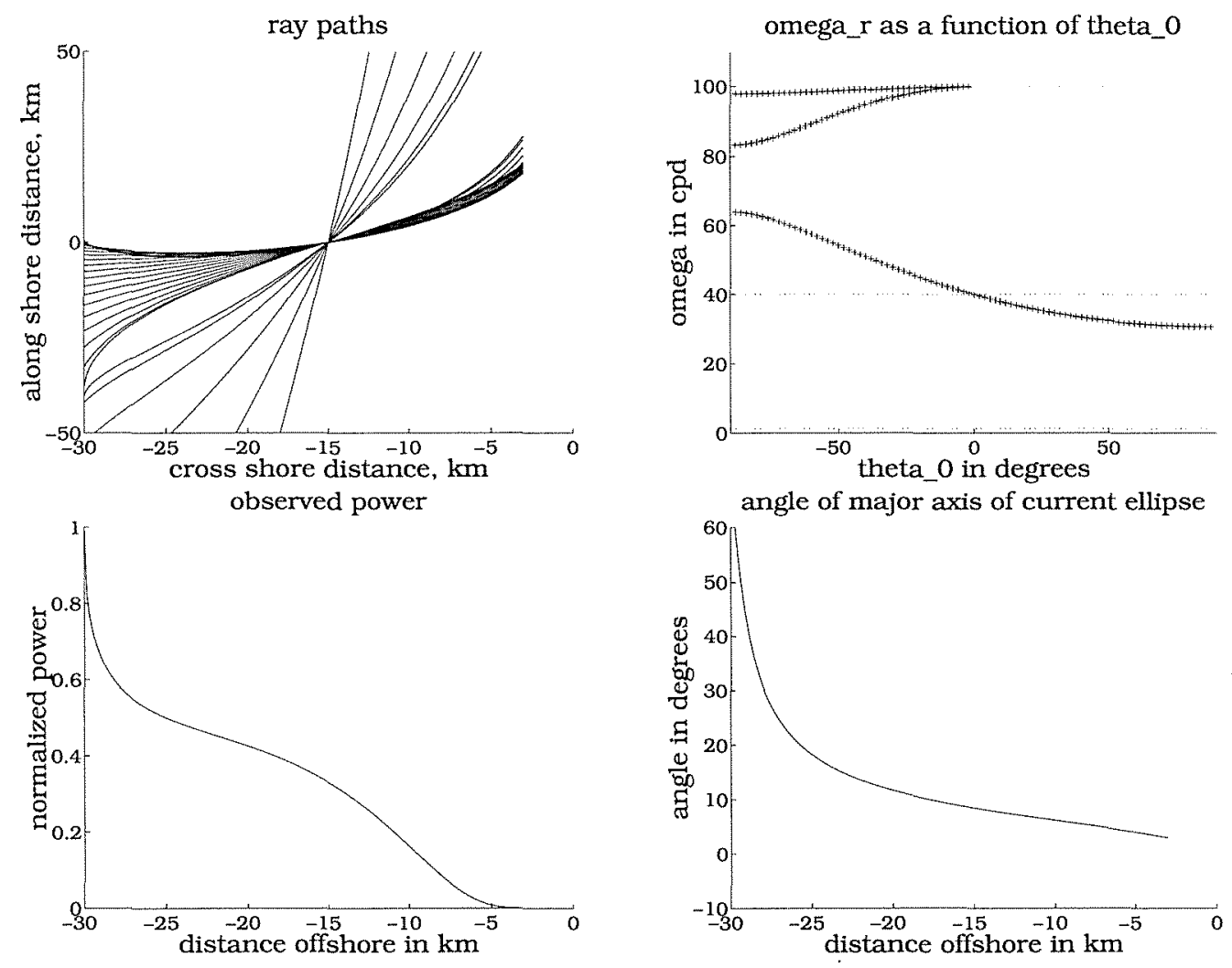

Figure 4.14: This is a plot of current meter observation model for the $\omega=40 \mathrm{cpd}$, $V=10 \mathrm{~cm} \mathrm{~s}^{-1}$ case. The panels are as in 4.13 . 
frequencies, and $\theta_{\text {elip }}$ is greater because the Doppler shifting around $\omega$ increases as because $\omega$ increased. Because of this increase in $\theta_{\text {elip }}$, the internal wave needs to move $40 \%$ of the way to the coast before the angle falls below $10^{\circ}$.

When the model is run with an alongshore current varying linearly between $V\left(x_{b}\right)=0$ and $V(0)=10 \mathrm{~cm} \mathrm{~s}^{-1}$, the angle of the major axis of the current ellipse to the cross shore direction, $\theta_{\text {elipm }}$, is much less than it was when $V$ was a constant $10 \mathrm{~cm} \mathrm{~s}^{-1}$. This is because all the rays have $\omega_{r}=\omega$ at $x_{b}$, so they all have the same initial amplitude. At $x=x_{b}, \theta_{\text {elip } \eta_{n}}$ is undefined because the current ellipse is a circle. As soon as the waves move into a region where $V$ is no longer zero and $\omega_{r}$ no longer equals $\omega$, the current ellipse becomes non-circular. This can be seen for $\omega=10 \mathrm{cpd}$ and $40 \mathrm{cpd}$ in figures 4.15 and 4.16. $\theta_{\text {elip }}$ jumps to about $35^{\circ}$ for both frequencies, but within $3 \%$ of the distance onshore, $\theta_{\text {elip }}$ falls to less than $1^{\circ}$. The initial high value of $\theta_{\text {elip }}$ is deceptive because the ellipticity is very low near $x_{b}$. As in the constant $V$ case, the effect of changing friction is minor, yielding less than a $10 \%$ change in angle. For both frequencies, $\theta_{\text {elip }}$ remains less than $3^{\circ}$ for the rest of the distance inshore. The angle is less than in the constant $V$ case until very near the coast. This result remains true as $V$ is increased to $60 \mathrm{~cm} \mathrm{~s}^{-1}$ in the sheared and non-sheared cases.

Thus the assumption that the initial energy on the ray scales as $\omega_{r}^{-2}$ dominates the solution, which is disturbing, for this assumption is based only on the GM spectrum, which is not meant to hold near the coast. This dependence on the initial condition is also disturbing because the choice of $x_{b}$ is arbitrary to the extent that it can be made greater, i.e. moved farther out into the ocean, with only minor modifications to the ray tracing theory to account for a different bottom slope. But then the $V\left(x_{b}\right)$ which sets $\omega_{r}$ and thus the initial amplitude of the ray would be different, and thus what would propagate onto the shelf would be different. This is a difficulty that can only be avoided by a knowledge of what sets the Garrett and Munk spectrum in the deep ocean or what determines the internal wave climate at $x_{b}$.

\subsection{Conclusions}

The ray tracing results for individual waves can be summarized in three points. 

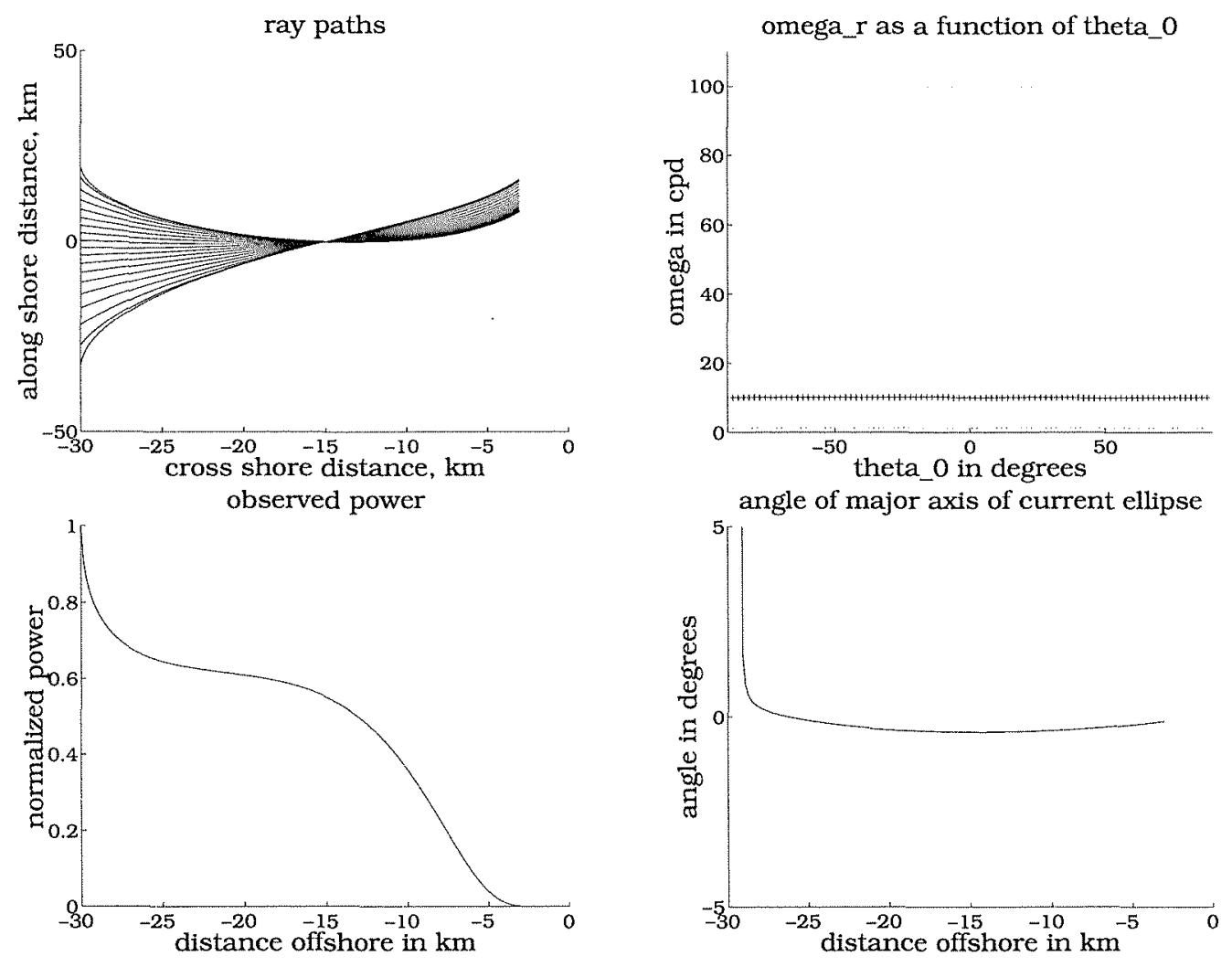

Figure 4.15: This is a plot of current meter observation model for the $\omega=10 \mathrm{cpd}$, $V\left(x_{b}\right)=10 \mathrm{~cm} \mathrm{~s}^{-1}, V(0)=0$ case. The panels are as in 4.13 except that the scale of the lower right hand plot of $\theta_{\text {elipm }}$ has been reduced. 

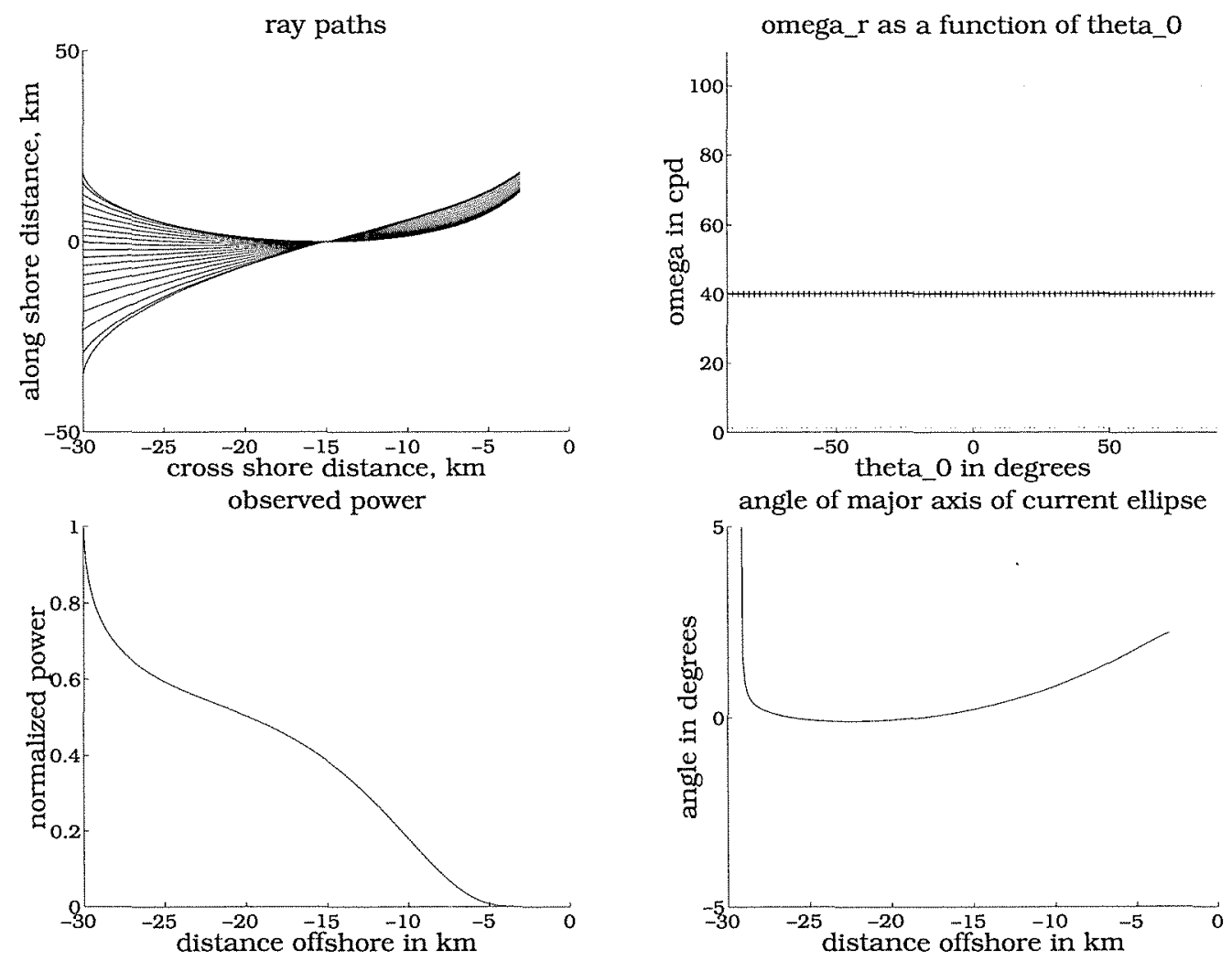

Figure 4.16: This is a plot of current meter observation model for the $\omega=40 \mathrm{cpd}$, $V\left(x_{b}\right)=10 \mathrm{~cm} \mathrm{~s}^{-1}, V(0)=0$ case. The panels are as in 4.13 except that the scale of the lower right hand plot of $\theta_{e l i p_{m}}$ has been reduced. 
- Unless the vertical mode assumption breaks down, or $\omega$ becomes greater than $N$ near the bottom, waves generated on the shelf cannot propagate into the deep ocean unless the initial wavevector points nearly directly offshore.

- In the absence of a horizontally sheared flow, all waves are dissipated as they approach the shore, and for reasonable values of friction, $x_{c} \leq K$, the amplitude of the wave never increases as the wave propagates onshore, removing the need to appeal to wave breaking in order to limit internal wave energy near the shore.

- A horizontally sheared flow can reflect waves which are traveling in one direction alongshore, while the same flow can cause a wave traveling in the opposite alongshore direction to be dissipated. A wave can thus be trapped between two isobaths by a sheared flow, while a wave moving in the other alongshore direction would be absorbed in the current or against the coast.

The results of integrating over all internal waves passing through a location can be summarized in a further four points.

- The ellipticity of a current ellipse at a given frequency is independent of the frequency for much of the shelf.

- The greater friction, or the wider the shelf, the more waves generated on the shelf will dominate the observations.

- Higher mode and higher frequency waves are more dissipated by friction across the shelf, and so the frequency spectrum of waves propagating from the shelf break should become redder onshore.

- A mean current at the shelf break will introduce an asymmetry in the internal wave field on the shelf primarily because the modeled internal wave amplitude at the shelf break depends on the wave's intrinsic frequency at the shelf break.

This analysis provides only an incomplete understanding of the propagation of linear internal waves onto a shelf. The most important phenomena that are not considered in this analysis are baroclinic mean flows, time varying mean flows, and mean flows which vary in the along shelf direction. Baroclinic flows, as shown in Olbers [1981], can cause critical layers across which internal waves can cross vertically in only one direction. This would dramatically alter a vertical modal structure. Time 
varying mean flows can change the frequency of an internal wave [Lighthill, 1978] and also lead to freely propagating waves generated by topographical irregularities,

e.g. Lamb [1994]. Alongshore variability in the mean flow would force alongshore variability in the internal waves. The waves could be focused or dispersed, leading to changes in the local internal wave power levels.

Even if the above mechanisms were perfectly understood, it would not be possible to understand the internal wave climate on the shelf without a better understanding of sources of internal waves on the shelf itself, and the nature of the internal waves propagating from the deep sea and shelf break onto the shelf.

\section{Acknowledgments}

This paper originated in Jay Austin's remark that internal waves should refract towards shore as surface gravity waves do. It was nurtured by many interesting talks with Lyn Harris, William Williams, Jay Austin, and Sarah Gille. It was greatly improved by the close readings given it by Steven Lentz. The work was funded by an Office of Naval Research fellowship and and Office of Naval Research AASERT fellowship, N00014-95-1-0746. Further support was provided through Office of Naval Research grant N00014-92-J-1528.

\subsection{Appendix A: The Internal Wave Bottom Boundary Condition}

In the derivations presented above, the bottom has been assumed to be locally flat, and it has been assumed that friction is an effect that does not make an $O(1)$ change to the modal structure of the internal wave. Ray tracing and energy conservation were used to examine the gradual evolution of the internal wave solutions as the waves shoaled and dissipated. In order to confirm these approximate solutions, and to demarcate the regions of their validity, it is useful to consider the true bottom boundary condition. The bottom boundary condition will be derived assuming that the boundary layer is "thin" in a sense described below. Then this bottom boundary condition will be used to define the sense in which friction is small, and in what 
senses the bottom in nearly flat. In appendix B, the boundary condition will be used to diagnose the energy loss in a caustic, where ray tracing breaks down.

To derive the bottom boundary conditions, it is necessary to modify equations (4.1a) and (4.1b) so that the horizontal momentum equations have vertical stress divergence terms:

$$
\begin{aligned}
& \frac{\partial u}{\partial t}-f v=-\frac{1}{\rho_{0}} \frac{\partial P}{\partial x}+\frac{\partial X}{\partial z} \\
& \frac{\partial v}{\partial t}+f u=-\frac{1}{\rho_{0}} \frac{\partial P}{\partial y}+\frac{\partial Y}{\partial z}
\end{aligned}
$$

Following the derivations of Clarke and Brink [1985], but without making any geostrophic or semi-geostrophic approximations, the volume conservation equation (4.1d) is integrated from the sea floor to the top of the boundary layer, which is defined as where the stress divergence becomes negligible. Thus

$$
\int_{-D}^{-D+\delta} \nabla \cdot \vec{u} d z=\left.w\right|_{-D} ^{-D+\delta}+\int_{-D}^{-D+\delta} \frac{\partial u}{\partial x} d z+\int_{-D}^{-D+\delta} \frac{\partial v}{\partial y} d z=0,
$$

where $\delta$ is the boundary layer thickness. No flow through the bottom implies that $w=-\frac{\partial D}{\partial x} u$ at the bottom. Using this condition and the chain rule, equation (A2) becomes

$$
\left.w\right|_{-D+\delta}+\left.\left[\frac{\partial}{\partial x}(D-\delta)\right] u\right|_{-D+\delta}+\int_{-D}^{-D+\delta} \frac{\partial u}{\partial x} d z+\int_{-D}^{-D+\delta} \frac{\partial v}{\partial y} d z=0 .
$$

This equation states that the vertical velocity at the top of the boundary layer is equal to the velocity of water flowing into the boundary layer plus the convergence and divergence of the horizontal flow in the bottom boundary layer. In order to evaluate this expression, it is necessary to assume that the pressure and its derivatives do not vary significantly through the boundary layer, so that the integrals of pressure and its derivatives through the boundary layer are the pressure and its derivatives at the bottom of the boundary layer times the boundary layer thickness, $\delta$. This is consistent with a nearly modal solution if $\delta \ll D M^{-1}$. Eliminating the velocities from (A3) for the pressure and stress evaluated at $z=-D$, assuming that $\delta$ does not vary in $x$, and substituting $P(x, z) \exp (i(l y-\omega t))$ for pressure (it is the only consistent form for a bathymetry that does not vary in $y$ ) leads to

$$
\begin{aligned}
c^{2} \frac{\partial P}{\partial z}+\alpha\left(\frac{\partial P}{\partial x}+\frac{l f}{\omega} P\right) & -\left(\delta \frac{\partial^{2} P}{\partial x^{2}}+\frac{\partial X}{\partial x}+\frac{l f}{\omega} \delta \frac{\partial P}{\partial x}-\frac{i f}{\omega} \frac{\partial Y}{\partial x}\right) \\
& -\left(-l^{2} \delta P+i l Y-\frac{l f}{\omega} \delta \frac{\partial P}{\partial x}-\frac{l f}{\omega} X\right) \quad \text { at } \quad z=-D
\end{aligned}
$$


where again $\alpha$ is the slope of the bottom. To evaluate the stress terms, a drag law must be chosen. As in Brink [1988], the drag law is chosen to be linear so that $X=\left.r u\right|_{-D}$ and $Y=\left.r v\right|_{-D}$ where $u$ and $v$ are the inviscid velocities. Thus the stress is:

$$
X=\left.\frac{-r}{\omega^{2}-f^{2}}\left(i \omega \frac{\partial P}{\partial x}+i f l P\right)\right|_{z=-D}
$$

and

$$
Y=\left.\frac{-r}{\omega^{2}-f^{2}}\left(-\omega l P-f \frac{\partial P}{\partial x}\right)\right|_{z=-D} .
$$

The stress term in equation (A4) can be shown to be larger than the terms multiplied by $\delta$ if $1 \ll r \omega^{-1} \delta^{-1}$. This is not in general the case for internal waves, and thus this derivation retains the terms containing $\delta$, unlike Brink [1988] and Clarke and Brink [1985]. The magnitude of $\delta$ will be discussed below. Equations (A4) and (A5) can be solved to give the bottom boundary condition

$$
c^{2} \frac{\partial P}{\partial z}+\alpha\left(\frac{\partial P}{\partial x}+\frac{l f}{\omega} P\right)+\frac{i r}{\omega}\left(1+\frac{i \delta \omega}{r}\right)\left(\frac{\partial^{2} P}{\partial x^{2}}-l^{2} P\right)=0 \quad \text { at } z=-D .
$$

With this boundary condition, one can determine when friction is small in the sense that the inviscid vertical modal solution is changed only slightly. Vertical modes are useful for they allow the field equation for the internal waves to be represented approximately as an ordinary differential equation, which makes the analysis in the body of the paper and in appendix B simpler.

To examine friction, the boundary condition for a flat bottom $(\alpha=0)$ is used,

$$
c^{2} \frac{\partial P}{\partial z}+\frac{i r}{\omega}\left(1+\frac{i \delta \omega}{r}\right)\left(\frac{\partial^{2} P}{\partial x^{2}}-l^{2} P\right)=0 \quad \text { at } \quad z=-D,
$$

along with a field equation for pressure outside the bottom boundary layer derived from equations (4.1)

$$
\frac{\partial^{2} P}{\partial x^{2}}-c^{2} \frac{\partial^{2} P}{\partial z^{2}}-l^{2} P=0
$$

The top boundary condition is a rigid lid, which implies no vertical pressure gradient at the top. The inviscid solutions $(r=\delta=0)$ to equation (A8) for a flat bottom are

$$
P=\cos \left(\frac{M \pi}{D} z\right) P(x) \quad M=1,2, \ldots \infty .
$$


Assuming that the solution to the problem with friction can be found by perturbing M slightly [Brink, 1988], the solution

$$
P=\cos \left(\frac{\pi}{D} M\left(1+\epsilon_{\text {fric }}\right) z\right) P(x)
$$

is substituted into equation (A7), and, using equation (A8) to evaluate $\partial^{2} P / \partial x^{2}-l^{2} P$, $\epsilon_{\text {fric }}$ is found to be

$$
\epsilon_{\text {fric }}=\frac{i r}{\omega D}\left(1+\frac{i \delta \omega}{r}\right) \quad \text { for } \quad|\epsilon| \ll 1 .
$$

$\epsilon_{\text {fric }}$ is small, and hence the modal solution approximately correct, if

$$
\frac{r}{\omega D} \ll 1 \text { and } \frac{\delta}{D} \ll 1,
$$

the latter condition already being necessary for the validity of equation (A6). Substituting the perturbed $M$ into equation (A8) leads to (again, simplifying with the assumption $\epsilon_{\text {fric }} \ll 1$ )

$$
\frac{\partial^{2} P}{\partial x^{2}}+\left[\frac{c^{2} \pi^{2} M^{2}}{D^{2}}\left(1+\frac{2 i r}{\omega D}-\frac{2 \delta}{D}\right)-l^{2}\right] P=0 .
$$

It can be seen from this equation that, to first order in $\delta D^{-1}$, the magnitude of $\delta$ only affects the horizontal wavelength of the wave, not the rate of dissipation per unit length of the wave, and that the horizontal wavelength is altered to $O\left(\delta D^{-1}\right)$ by $\delta$.

So what is $\delta$ ? It is in general less than the Ekman depth. For a constant eddy viscosity model,

$$
\delta=\sqrt{\frac{A}{\omega}},
$$

where $\mathrm{A}$ is an eddy viscosity which may depend on the mean current fields, surface waves, and many other disparate factors. For more sophisticated treatments of this issue, see Sanford and Grant [1987] and Trowbridge and Madsen [1984].

The effect of the sloping bottom is interesting, mathematically. The flat bottom solution, (A9), meets the boundary condition (A6), but only satisfies the field equation (A8) to order $\alpha c^{-1}$. Because of this, while (A8) captures the local wavelike nature of the solution, it does not capture the shoaling caused by topography. This is why section 4.4, which solves for the evolution of wave amplitude, uses energy conservation techniques to find the amplitude instead of analyzing (A8). It is possible, 
however, to cast the field equation as an ordinary differential equation for the amplitude of a flat bottom vertical mode and to include the effect of topography on the amplitude of the vertical mode in the ordinary differential equation. This is useful because in doing so one finds when the vertical structure over a sloping bottom can be approximated by the vertical modal structure for a flat bottom. In order to do this, a scattering technique will be used. Again, the bottom slope is assumed to be constant, and the depth will be of the form $D=-\alpha x$.

The field equation (A8) is converted into an ordinary differential equation for the amplitude of a flat bottom vertical mode by multiplying the equation for the flat bottom vertical mode $j, \hat{P}(z)$,

$$
\begin{array}{r}
\frac{\partial^{2} \hat{P}}{\partial z^{2}}+\frac{j^{2} \pi^{2}}{\alpha^{2} x^{2}} \hat{P}=0 \\
\frac{\partial \hat{P}}{\partial z}=0 \quad @ \quad z=0,-D
\end{array}
$$

by the full solution to (A8), $P(x, z)$, adding this to (A8) times $c^{-2} \hat{P}$, and integrating over the depth to obtain

$$
0=\int_{-D}^{0} d z\left(P \frac{\partial^{2} \hat{P}}{\partial z^{2}}+\frac{j^{2} \pi^{2}}{\alpha^{2} x^{2}} \hat{P} P+c^{-2} \hat{P} \frac{\partial^{2} P}{\partial x^{2}}-c^{-2} l^{2} \hat{P} P-\hat{P} \frac{\partial^{2} P}{\partial z^{2}}\right) .
$$

Using the boundary conditions (A15b) and (A6), (A16) can be written as

$$
0=\alpha \hat{P}\left(\frac{l f}{\omega} P-\frac{\partial P}{\partial x}\right)+\int_{-D}^{0} d z\left[\left(c^{2} \frac{j^{2} \pi^{2}}{\alpha^{2} x^{2}}-l^{2}\right) \hat{P} P+\hat{P} \frac{\partial^{2} P}{\partial x^{2}}\right] .
$$

In order to examine the evolution of a vertical mode $j$ in a wave whose energy is primarily in mode $M$, solutions for $P$ and $\hat{P}$ of the form

$$
\begin{aligned}
& \hat{P}=\cos \left(\frac{j \pi}{\alpha x} z\right) \\
& P=P^{M}(x) \cos \left(\frac{M \pi}{\alpha x} z\right)+\sum_{n \neq M} \epsilon_{n}(x) \cos \left(\frac{n \pi}{\alpha x} z\right)
\end{aligned}
$$

are chosen. If these solutions are substituted into (A17), the resulting equations can be consistently grouped only if $\epsilon_{n}$ scales as $\alpha c^{-1}$. The leading orders of the equation for the evolution of $P^{M}$ is found by setting $j=M$ :

$$
\underbrace{\frac{\partial^{2} P^{M}}{\partial x^{2}}+\left(c^{2} \frac{M^{2} \pi^{2}}{\alpha^{2} x^{2}}-l^{2}\right) P^{M}}_{O(1)}+\underbrace{\frac{1}{x} \frac{\partial P^{M}}{\partial x}}_{O\left(\alpha c^{-1}\right)}+O\left(\alpha^{2} c^{-2}\right)=0 .
$$




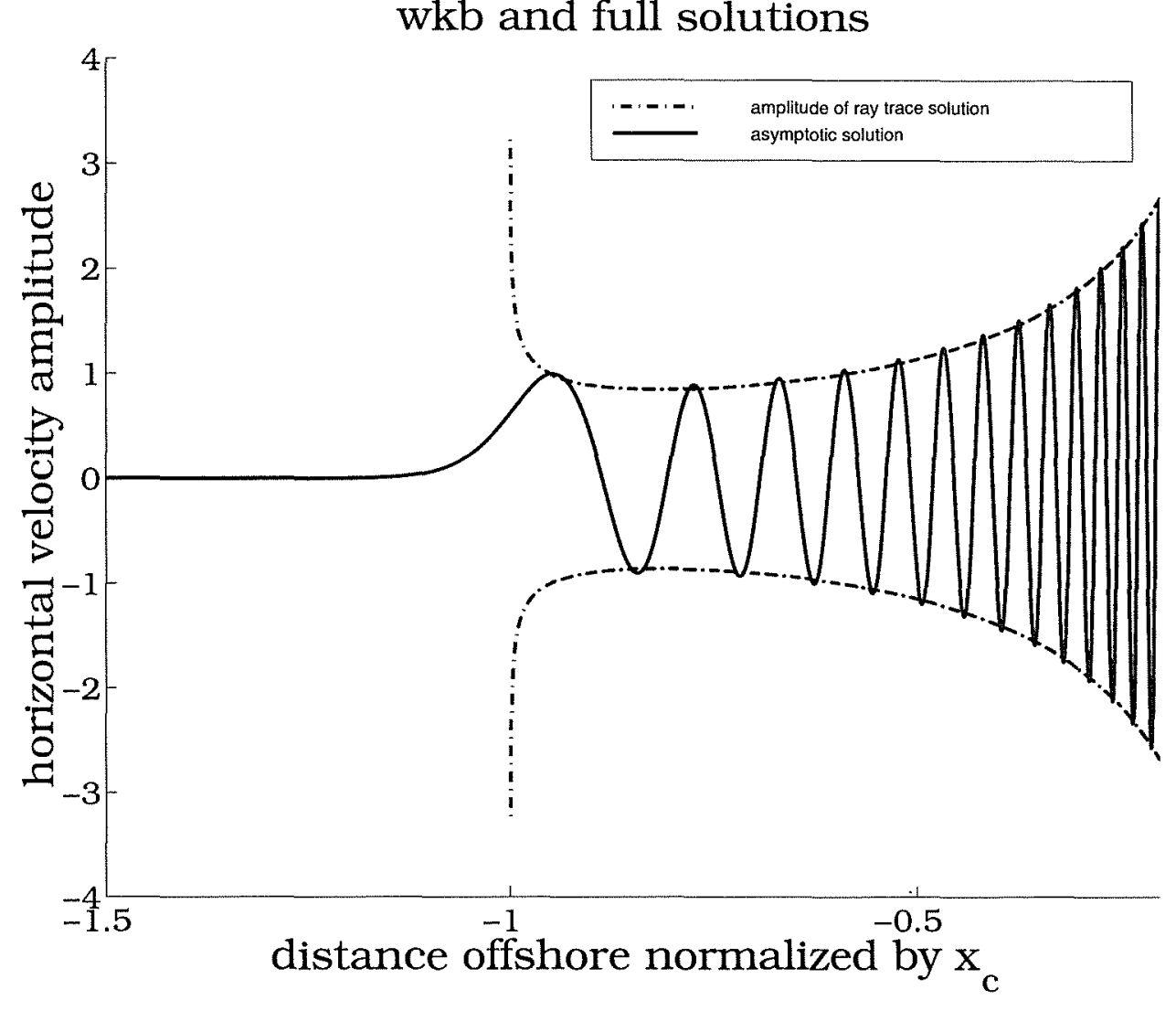

Figure A1: This is the horizontal velocity solution predicted by (A13) (solid line) and the wave amplitude predicted with WKB methods in (4.24) (dashed line). The cross shelf distance has been normalized by $x_{c}$, in this case $60 \mathrm{~km} . M=1, \omega=10 \mathrm{cpd}$, $\alpha=5 \times 10^{-3}$ and $N \approx 100 \mathrm{cpd}$.

This equation governs the growth in amplitude of the internal wave as it crosses the shelf as long as mode $M$ dominates the vertical structure of $P$. It is shown below that mode $\mathrm{M}$ dominates the vertical structure only as long as $\alpha c^{-1}$ is small, and so (A19) is valid only when $\alpha c^{-1}$ is small, but is accurate to $O\left(\alpha^{2} c^{-2}\right)$. The solution to (A19) is evaluated numerically and plotted in figure A1 along with the inviscid amplitude predicted by (4.24), and the agreement is good for $\alpha c^{-1}=0.05$, which is appropriate for a $10 \mathrm{cpd}$ wave with $\alpha=5 \times 10^{-3}$ and $N \approx 100 \mathrm{cpd}$. 
The sizes of the $\epsilon_{n}$ can be shown to be of order $\alpha c^{-1}$ by setting $j \neq M$ to obtain

$$
\begin{aligned}
& \underbrace{\frac{\partial^{2} \epsilon_{n=j}}{\partial x^{2}}+\left(c^{2} \frac{M^{2} \pi^{2}}{\alpha^{2} x^{2}}-l^{2}\right) \epsilon_{n=j}+\mathcal{C}_{1}(n, M) x^{-1} \frac{\partial P^{M}}{\partial x}+\mathcal{C}_{2}(n, M) x^{-2} P^{M}} \\
& O\left(\alpha c^{-1}\right) \\
& +O\left(\alpha^{2} c^{-2}\right)=0
\end{aligned}
$$

where $\mathcal{C}_{1}$ and $\mathcal{C}_{2}$ depend only on $n$ and $m$. (A20) is non-resonantly forced by terms containing $P^{M}$ of order $\alpha c^{-1}$, and hence it is consistent for all $\epsilon_{n}$ to be $O\left(\alpha c^{-1}\right)$. Thus the vertical structure of $P$ is dominated by mode $M$ when $\alpha c^{-1}$ is small.

\subsection{Appendix B: The Caustic at $x_{c}$}

Ray tracing fails at the caustic where the ray approaches $-x_{c}$. Thus the ray tracing theory developed in the main text cannot predict the amplitude of the wave leaving the caustic given the amplitude of the wave entering the caustic. In the absence of friction, of course, the solution is easy: all waves are reversible, thus the outgoing wave has the same energy flux as the incoming wave, but oriented away from the caustic. The calculation that follows predicts the amplitude of a ray leaving a caustic as a function of the amplitude and orientation of the incoming ray, the bathymetry, and the strength of friction.

The derivation starts with (A13). It is useful to write (A13) as

$$
k^{2}=\frac{\zeta^{2}\left(1+\frac{2 \epsilon_{c} x_{c}}{x}\right)}{x^{2}}-\frac{\zeta^{2}}{x_{c}^{2}}
$$

and

$$
\frac{\partial^{2} P}{\partial x^{2}}+k^{2} P=0
$$

where

$$
\begin{aligned}
\zeta & =\frac{M \pi c}{\alpha} \\
\epsilon_{c} & =\frac{i r}{\omega \alpha x_{c}}\left(1+\frac{i \delta \omega}{r}\right)
\end{aligned}
$$


and $x_{c}$ is as before. $\zeta$ can be thought of as a scaled characteristic slope of an internal wave, and $\epsilon_{c}$ can be thought of as the strength of friction at the caustic. Approximations in the main text limit $\zeta \gg 1$ and $\epsilon_{c} \ll 1$. Following Lighthill [1978], $k^{2}$ can be expanded as a Taylor series around the caustic in the inviscid problem, where $x=-x_{c}$ :

$$
k^{2}=\left(2+6 \epsilon_{c}\right) \frac{\zeta^{2}}{x_{c}^{3}}\left(x+x_{c}\right)+\frac{\zeta^{2} \epsilon_{c}}{x_{c}^{2}} .
$$

This implies a solution to $P$ of

$$
P=A i\left(-\left(\left(2+6 \epsilon_{c}\right) \frac{\zeta^{2}}{x_{c}^{3}}\right)^{\frac{1}{3}}\left(x+x_{c}\left(1+\epsilon_{c}\right)\right)\right)
$$

where $A i$ is the Airy function. This is a good approximation of the solution to $\mathrm{P}$ as long as the Taylor expansion of $k^{2}$ is valid, which it is when

$$
\frac{2}{3} x_{c} \gg x+x_{c}
$$

(B4) can be analyzed with WKBJ / ray tracing methods when

$$
x+x_{c}>2^{-\frac{1}{3}} \zeta^{-\frac{2}{3}} x_{c}
$$

[Lighthill, 1978]. Where (B4) (and thus (B1)) can be ray traced, the ray solutions derived earlier in the paper can be equated to the rays leaving and entering the caustic, and thus the amplitude of a wave leaving the caustic can be found from the amplitude of a wave entering a caustic. It is best to fit the rays to the Airy function approximation inside the region where the WKBJ approximation to (B4) is valid, or, from $(\mathrm{B} 6)$, when

$$
x_{\text {good }}=\beta 2^{-\frac{1}{3}} \zeta^{-\frac{2}{3}} x_{c}-x_{c}
$$

where $\beta$ is an $O(1)$ positive constant. The choice of $\beta$ is somewhat arbitrary, but as it gets larger, the WKBJ approximation to the Airy function becomes more accurate but the Airy function approximation to (B1) becomes less accurate. Both the Airy function approximation to (B1) and the WKBJ approximation to the Airy function are valid at $x_{\text {good }}$ when both $(\mathrm{B} 6)$ and (B5) are true, or when

$$
\frac{2^{\frac{4}{3}}}{3 \beta} \gg \zeta^{-\frac{2}{3}}
$$


The WKBJ/Ray Tracing approximation to (B4) is

$$
\begin{aligned}
P_{\text {airyWKBJ }}=\frac{1}{\sqrt{\pi}}\left[\left(1+6 \epsilon_{c}\right) \zeta^{2} x_{c}^{-3}\right]^{-\frac{1}{12}}\left[x+x_{c}\left(1+\epsilon_{c}\right)\right]^{-\frac{1}{4}} \\
\cos \left[\frac{2}{3}\left(1+6 \epsilon_{c}\right)^{\frac{1}{2}} \zeta x_{c}^{-\frac{3}{2}}\left(x+x_{c}\left(1+\epsilon_{c}\right)\right)^{\frac{3}{2}}-\frac{\pi}{4}\right]
\end{aligned}
$$

[Lighthill, 1978]. The ray tracing approximation to the incoming and outgoing waves is, locally,

$$
P_{\text {rays }}=P_{i n} e^{-i k x}+P_{\text {out }} e^{i k x}
$$

where $P_{\text {in }}$ is the amplitude of the wave entering the caustic, $P_{\text {out }}$ the amplitude of the wave leaving the caustic, and $k$ is the $x$ wavenumber from (B1a). (B10) can be solved for the amplitude of $P_{\text {in }}$ and $P_{\text {out }}$ as a function of $P_{\text {rays }}$ and its derivative in $x$, and then $P_{a i r y W K B J}$ can be substituted for $P_{\text {rays }}$, giving

$$
\left|P_{i n}\right|=\quad 0.5\left|P_{\text {airyWKBJ }}+i k^{-1} \frac{\partial P_{\text {airy } W K B J}}{\partial x}\right|
$$

and

$$
\left|P_{\text {out }}\right|=\quad 0.5\left|P_{\text {airyWKBJ }}-i k^{-1} \frac{\partial P_{\text {airy } W K B J}}{\partial x}\right| .
$$

Evaluating the ratio of $P_{\text {out }}$ to $P_{i n}$ at $x_{\text {good }}$ gives the amplitude lost in the caustic and between the caustic and $x_{\text {good }}$. It is sensitive to $\beta$ only insofar as some energy is lost between the caustic and $x_{\text {good }}$. A $\beta$ of 1.5 is found to work well in numerical solutions of (B1).

The expression gained from substituting (B7), (B4), and (B1a) into (B11) is unwieldy, but the amplitude loss, the ratio of $\left|P_{\text {out }}\right|$ to $\left|P_{i n}\right|$, can be easily contoured. Because $\epsilon_{c}$ contains $\alpha$ and $\omega$ in a different combination, $(\omega \alpha)$, than $\zeta$ contains $\alpha$ and $\omega$ (approximately $\left(\omega \alpha^{-1}\right)$ ), it makes no sense to hold $\epsilon_{c}$ constant as $\zeta$ is varied. Thus for figure $\mathrm{B} 1$ the amplitude loss is plotted with $\epsilon_{c}$ varying as it would when $\zeta$ varies because $\omega$ varies and $\alpha$ is constant, and in figure B2 $\epsilon_{c}$ varies as it would when $\zeta$ varies because $\alpha$ varies and $\omega$ is constant. 


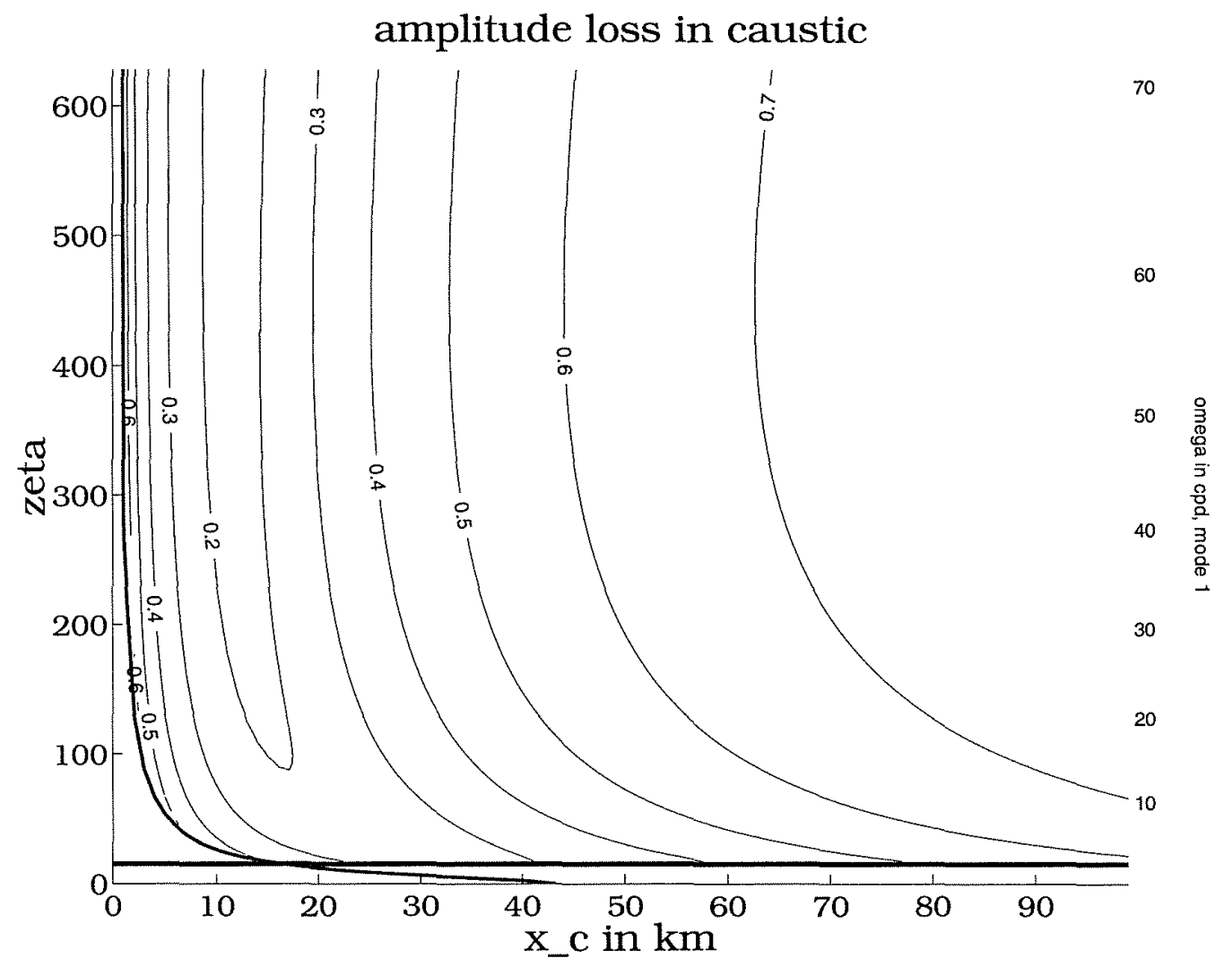

Figure B1: A plot of the amplitude lost in a caustic as given by $\left|P_{\text {out }} P_{\text {in }}^{-1}\right| . \epsilon_{c}$ is calculated as a function of $\zeta$ and $x_{c}$ assuming that $\alpha$ and $r$ are constant and $\omega$ varies. $\omega$ for a mode 1 wave is printed on the right hand side. Since $\zeta \propto M^{-1}$, a mode 2 wave has a $\zeta$ half that of a mode one wave. The thick, nearly vertical line delimits where the solution is invalid because $\epsilon_{c}$ is no longer small, and the thick horizontal line delimits where (B8) fails. 


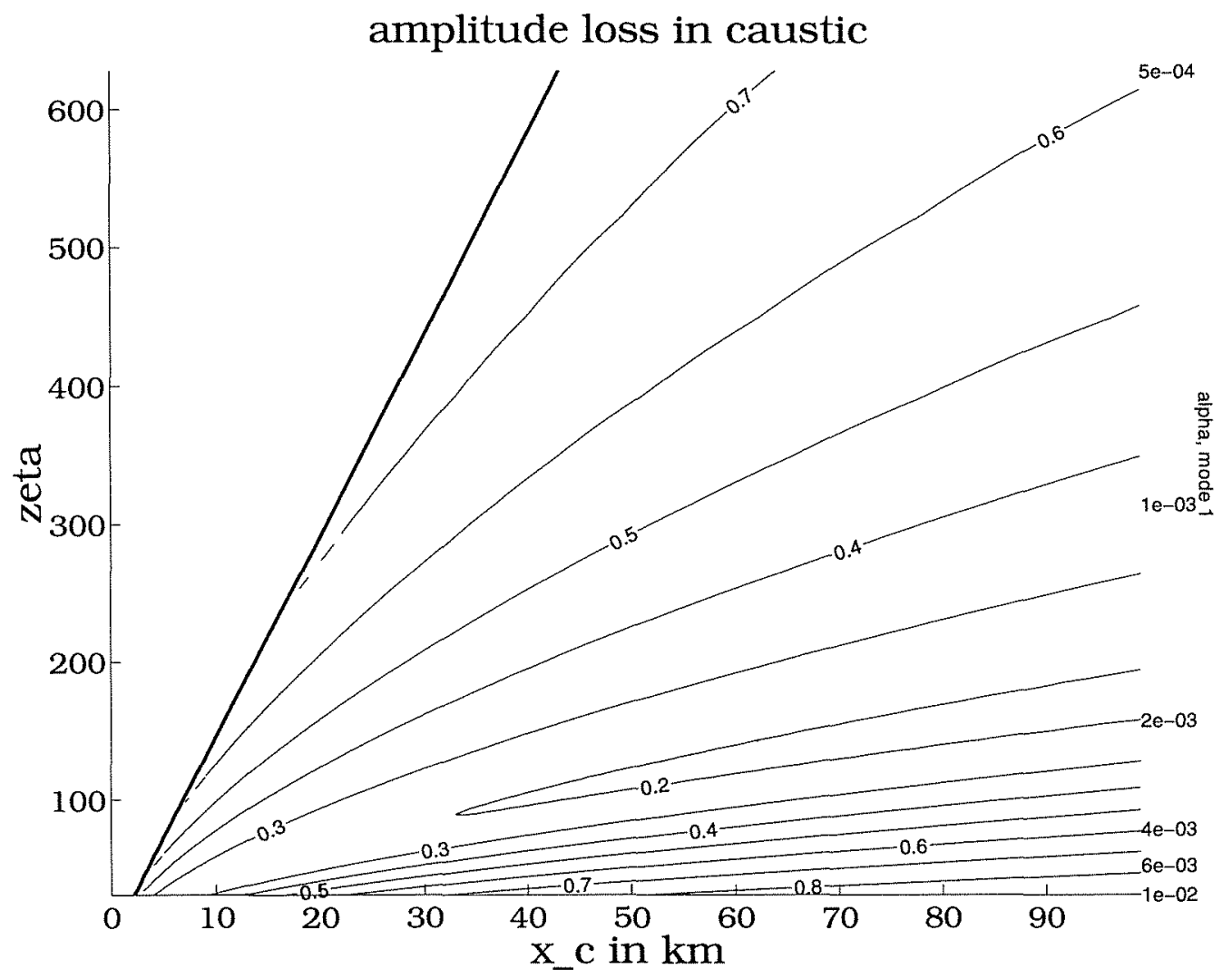

Figure B2: A plot of the amplitude lost in a caustic as given by $\left|P_{\text {out }} P_{\text {in }}^{-1}\right| . \epsilon_{c}$ is calculated as a function of $\zeta$ and $x_{c}$ assuming that $\omega$ and $r$ are constant and $\alpha$ varies. $\alpha$ for a mode 1 wave is printed on the right hand side. Since $\zeta \propto M^{-1}$, a mode 2 wave has a $\zeta$ half that of a mode one wave. The thick, line delimits where the solution is invalid because $\epsilon_{c}$ is no longer small. 


\subsection{Bibliography}

Brink, K. H., On the effect of bottom friction on internal waves, Continental Shelf Research, 8(4), 397-403, 1988.

Chapman, D. C. and M. C. Hendershott, Scattering of internal waves obliquely incident upon a step change in bottom relief, Deep Sea Res., 28A(11), 1323-1338, 1981.

Clarke, A. J. and K. H. Brink, The response of stratified, frictional flow of shelf and slope waters to fluctuating large-scale low-frequency wind forcing., J. Phys. Oceanogr., 15, 439-453, 1985.

Garrett, C. and W. Munk, Space-Time Scales of Internal Waves, Geophys. Fluid Dyn., $3,225-264,1972$.

Godin, G., Tides, Centro de Investigaciòn Cientifica y de Edudcaciòn Superior de Ensenada, Ensenada, Baja California, Mexico, 1988.

Kunze, E., Near-Inertial Wave Propagation in Geostrophic Shear, J. Phys. Oceanogr., $15,544-565,1985$.

Lamb, K. G., Numerical experiments of internal wave generation by strong tidal flow across a finite amplitude bank edge, J. Geophys. Res., 99(C1), 843-864, 1994.

LeBlond, P. H. and L. A. Mysak, Waves in the Ocean, Elsevier Scientific Publishing Co, Amsterdam, 1978.

Lighthill, J., Waves in Fluids, Cambridge University Press, Cambridge, 1978.

McKee, W. D., Internal-inertial Waves in a Fluid of Variable Depth, Proc. Camb. Phil. Soc., 73, 205-213, 1973.

Olbers, D. J., The Propogation of Internal Waves in a Geostrophic Current, J. Phys. Oceanogr., 11, 1224-1233, 1981.

Sanford, L. P. and W. D. Grant, Dissipation of Internal Wave Energy in the Bottom Boundary Layer on the Continental Shelf, J. Geophys. Res., 92(C2), 1828$1844,1987$.

Trowbridge, J. and O. S. Madsen, Turbulen Wave Boundary Layers: 1. Model formulation and First Order Solution, J. Geophys. Res., 89(C5), 7989-7997, 1984.

Wunsch, C., Progressive internal waves on slopes, J. Fluid Mech., 35, 131-144, 1969. 


\title{
Chapter 5
}

\section{Observations of High Frequency Internal Waves in the CODE}

\section{Region}

\begin{abstract}
Current meter data from the second Coastal Ocean Dynamics Experiment (CODEII) for July 1982 are analyzed for internal waves in the 6 to 40 cycles per day (cpd) frequency band. It is found that the wave field is anisotropic, and that the current ellipses are oriented in approximately the cross-isobath direction. The square of the ratio of the major to minor axes of the current ellipses (the "ellipticity") is consistent with a continuum of internal waves propagating onshore, but is not consistent with a single wave propagating onshore. The reduction of internal wave energy across the shelf is consistent with propagation from the deep ocean or shelf break, as is the correlation between vertical velocities and velocities parallel to the minor axis. However, there is evidence for the generation of additional internal wave energy on the shelf in the evolution of the current ellipses across the shelf, and in the "bluing" of the internal wave spectra across the shelf. Internal wave energy levels on the shelf are elevated relative to Garrett and Munk [1972] levels, and the first vertical mode dominates the internal wave spectra on the shelf.
\end{abstract}




\subsection{Introduction}

In the deep ocean, away from horizontal and vertical boundaries, the high frequency internal wave spectrum is well modeled by the Garrett \& Munk spectrum [Garrett and Munk, 1972, hereafter GM]. In shallow coastal regions there is no such universal description of the internal wave field. Thus it is useful and interesting to examine the internal wave climate at a particular shelf location. The current meters and thermistors deployed during the Coastal Ocean Dynamics Experiment (CODE) allow one to examine how the high frequency internal wave spectrum changes across the continental shelf. This is done for the central current meter array of the CODE II experiment for the month of July, 1982, and an accompanying study in chapter 4, (hereafter referred to as $\mathrm{PB}$ ) models linear internal wave propagation from the shelf break to the coast using a GM spectrum as the deep ocean initial condition. To the extent that the observations and theory can be compared and differ, it gives an idea of how much of the internal wave energy on the shelf is generated on the shelf, and how much propagates in from the ocean. (A detailed comparison of PB and the data is impossible because the data are unable to resolve the horizontal wave number spectrum of the internal waves, and the internal wave spectrum in the ocean adjacent to the shelf is also unknown.)

For the CODE region, high frequency waves have been defined, somewhat arbitrarily, as those with frequencies between 6 and 40 cycles per day. This range of frequencies is between the highest frequency of critical topographical reflection of internal waves $(6 \mathrm{cpd})$ and the lowest buoyancy frequency observed on the shelf for the time of the analysis ( $40 \mathrm{cpd}$ ). The month of July, 1982, was chosen because the stratification was relatively constant throughout the month, and the hydrographic structure was generally simple.

The high frequency internal wave climate on the shelf is interesting not only in its own right, but because it can affect diapycnal mixing [Sandstrom and Elliott, 1984; Sanford and Grant, 1987], and the propagation of acoustic energy on the shelf [Lynch et al., 1996]. It has been studied by several authors, including Gordon [1978] who analyzed internal waves using current meter data taken off Spanish Sahara at $21^{\circ} \mathrm{N}$, $17^{\circ} \mathrm{W}$ as part of the 1974 JOINT-1 experiment. That shelf has a bottom slope, $2 \times 10^{-3}$, between those typical of the east and west coasts of North America. He concluded from an empirical orthogonal function analysis that most of the energy is 
in the first mode internal waves, and that those waves propagated toward the shore. He claimed that energy dissipation was dominated by the effects of shoaling as the waves entered shallow water, and by the subsequent non-linear dissipative effects of wave breaking.

Another analysis of internal wave data by Howell and Brown [1985] is of interest because it was done at the same site as the present analysis. Howell and Brown analyzed six internal wave soliton events which occurred during a six day period in April of 1981. They conclude that they did observe solitons with a period of about 25 minutes, and that the events corresponded well with two layer soliton theory. Howell and Brown's conclusions would have to be included in any more complete analysis of internal waves on the shelf.

\subsection{Internal Wave Background}

Garrett and Munk [1972] codified the deep water internal wave spectrum, and their scheme has proven to be surprisingly robust away from horizontal boundaries, vertical boundaries, and the equator [Wunsch, 1976]. There is no reason to expect that it will be correct on or near the shelf. However, since deep ocean internal waves may propagate onto the shelf, and since whatever processes maintain the deep ocean at the GM spectrum may also drive the coastal internal wave spectrum locally, the GM spectrum makes a useful point of reference for any observed spectrum. In PB the propagation of a GM spectrum onto the shelf is explicitly modeled, but whatever non-linear processes equilibrate the GM spectrum in the deep ocean are not considered.

The GM spectral power density is, for horizontal currents observed by a current meter which moves with the sub-inertial current,

$$
\left\langle u^{2}\right\rangle+\left\langle v^{2}\right\rangle \text { per unit frequency }=\left(4 E b^{2} N_{0}\right) N f \omega^{-3} \frac{\omega^{2}+f^{2}}{\sqrt{\omega^{2}-f^{2}}},
$$

where $\left(4 E b^{2} N_{0}\right)$ is a scale power spectral density of $2.2 \mathrm{~m}^{2} \mathrm{~s}^{-1} \cdot\left\langle u^{2}\right\rangle$ and $\left\langle v^{2}\right\rangle$ are the horizontal current variances, and $N, f$, and $\omega$ are the local buoyancy, inertial, and internal wave frequencies. When $\omega^{2} \gg f^{2}$, the power spectrum can be simplified to

$$
\left\langle u^{2}\right\rangle+\left\langle v^{2}\right\rangle \text { per unit frequency }=\left(4 E b^{2} N_{0}\right) N f \omega^{-2}
$$


with very little error.

Wunsch and McKee examined internal waves propagating in a uniformly stratified wedge [McKee, 1973; Wunsch, 1968, 1969]. The McKee solutions show that incoming internal wave crests turn to parallel the beach in the same way that surface gravity waves do. They also derive the slope $c$ for critical internal wave reflection off the bottom:

$$
c=\sqrt{\frac{\omega^{2}-f^{2}}{N^{2}-\omega^{2}}}
$$

If the bottom slope is steeper than $c$, the wave will be reflected back to deeper water. If the slope is less than $0.5 c$, the bottom appears locally flat, and if the slope is nearly equal to $c$, a region of strong shear will exist near the bottom (c.f. PB).

\subsection{Topography and Coordinates}

The CODE region, which is described in Beardsley and Lentz [1987], is centered around $123^{\circ} 30 \mathrm{~W}$ and $38^{\circ} 30 \mathrm{~N}$, between Pt. Arena and Pt. Reyes, California. Figure 5.1 shows the location of the current meter moorings used in the present analysis. The coastline is straight for about $75 \mathrm{~km}$, the straight portion being approximately centered on the central ("C") moorings. The alongshore and cross-shelf directions are defined by the mean orientation of the coast around the central array: the crossshore axis points to $47^{\circ}$ true, and the alongshore axis points to $317^{\circ}$ true. [Beardsley, Limeburner and Rosenfeld, 1985]. The shelf break, however, is not parallel to the shore. A coordinate system defined by the $365 \mathrm{~m}$ isobath is rotated by about $17^{\circ}$ clockwise from the coordinate system defined at the shelf.

The shelf break is at about 200 meters, and the slope of the shelf averages $5 \times 10^{-3}$ between the $\mathrm{C} 4$ and $\mathrm{C} 3$ (130 and 90 meters respectively), and $5 \times 10^{-2}$ at $\mathrm{C} 5$. C5 is in 365 meters of water, beyond the shelf break at 200 meters.

Wunsch [1969] solves for the characteristic slope of an internal wave. If the bottom slope is greater then the characteristic slope of an internal wave, the wave can no longer be represented with vertical modes (PB). Since much of the analysis here depends on the existence of vertical modes, the analysis will be restricted to frequencies whose characteristic slope is everywhere greater than the bottom slope. 


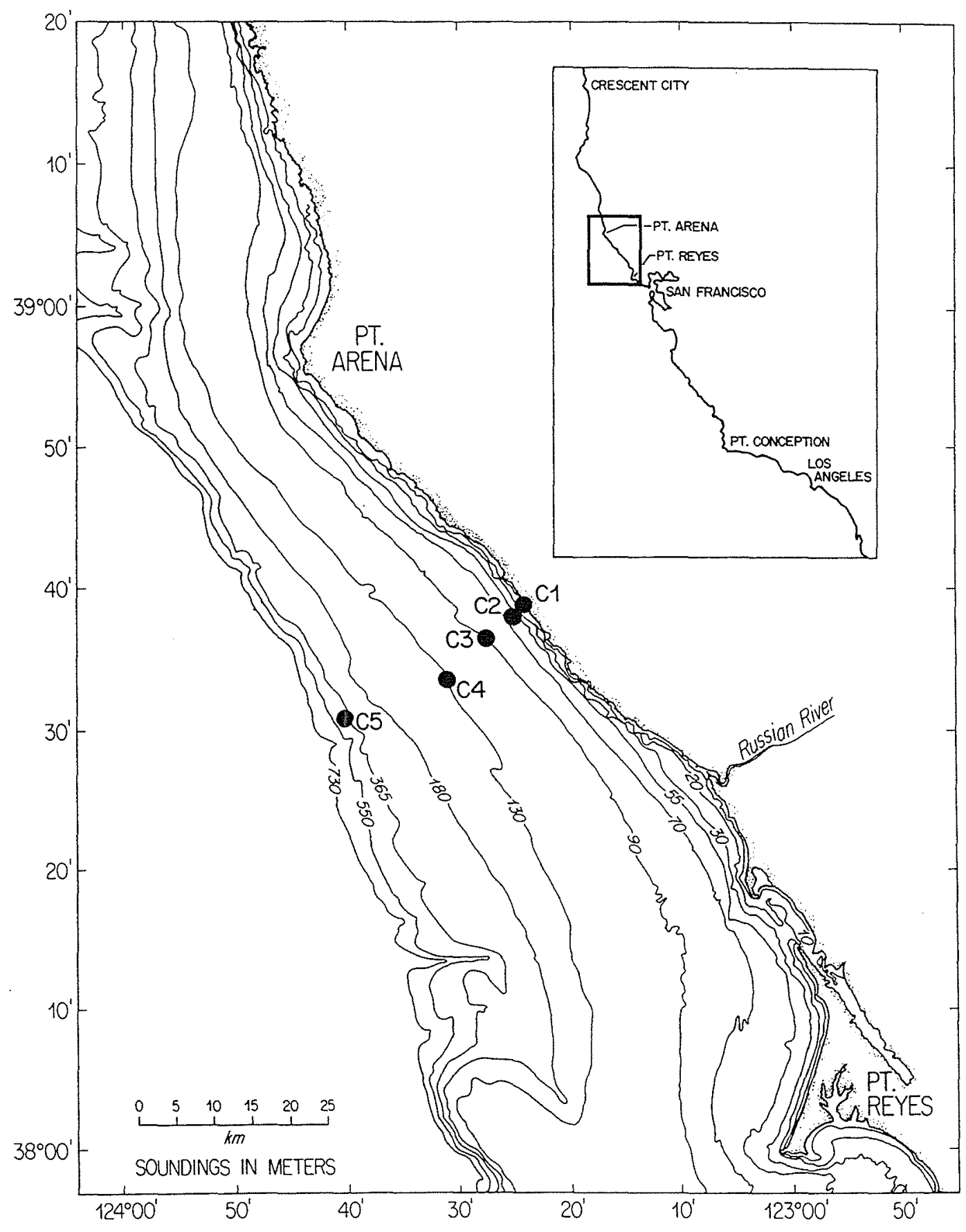

Figure 5.1: The CODE experimental region, showing the central line of moorings from the CODE II experiment. The C5, C4, and C3 moorings are used herein. 
This is true when

$$
\omega>\sqrt{\frac{f^{2}+\alpha^{2} N^{2}}{1+\alpha^{2}}} \approx \sqrt{f^{2}+\alpha^{2} N^{2}},
$$

where $\alpha$ is the bottom slope. An $\mathrm{N}$ of $100 \mathrm{cpd}$, an $f$ of $1.24 \mathrm{cpd}$, and a maximum bottom slope on the shelf break of $6 \times 10^{-2}$ restricts the analysis to frequencies greater then 6 cycles per day. This also avoids internal waves at the tidal and inertial frequencies, whose forcing mechanisms are likely to be different than the forcing mechanisms of the higher frequency internal waves. The critical frequency on the shelf, with its slope of $5 \times 10^{-3}$, is only $5 \%$ higher than $f$, and so is not near the frequencies analyzed here.

\subsection{Hydrography}

The month of July, 1982, was chosen for analysis because the hydrography had a simple relationship between temperature and density, which allows the calculation of density, $\sigma$, from the temperatures, $T$, observed at the current meters. The overall hydrography is typified by figure 5.2, an average for the April to July upwelling season. It is similar to the two sections taken in July 16th and 19th, 1982, and the many other CTD casts taken in July [Huyer, Fleischbein and Schramm, 1983].

A relation between temperature and potential density was formed from the CTD casts taken during July in the CODE region on the shelf and shelf break. Only data from less than 400 meters were used to obtain the relation

$$
\begin{aligned}
& \sigma=-0.1781 T+27 \quad T>7.74^{\circ} \mathrm{C} \\
& \sigma=-0.2633 T+28 \quad 7.74^{\circ} \mathrm{C}<T<9.212^{\circ} \mathrm{C} \\
& \sigma=-0.1975 T+28 \quad T>9.212^{\circ} \mathrm{C}
\end{aligned}
$$

This includes both the effect of the nonlinearity of the equation of state and the observed T/S relation. The RMS difference between (5.5) and the density computed with the full equation of state is $0.085 \mathrm{~kg} \mathrm{~m}^{-3}$. To provide confidence in this empirical relation, the buoyancy frequency from the CTD sections is plotted as computed from the full equation of state and as computed from equation 5.5 (figure 5.3). Though not a rigorous test, since it uses the same data as used to derive the $\sigma / \mathrm{T}$ relation, it is reassuring that there are few outliers. This $\sigma / \mathrm{T}$ relation allows 

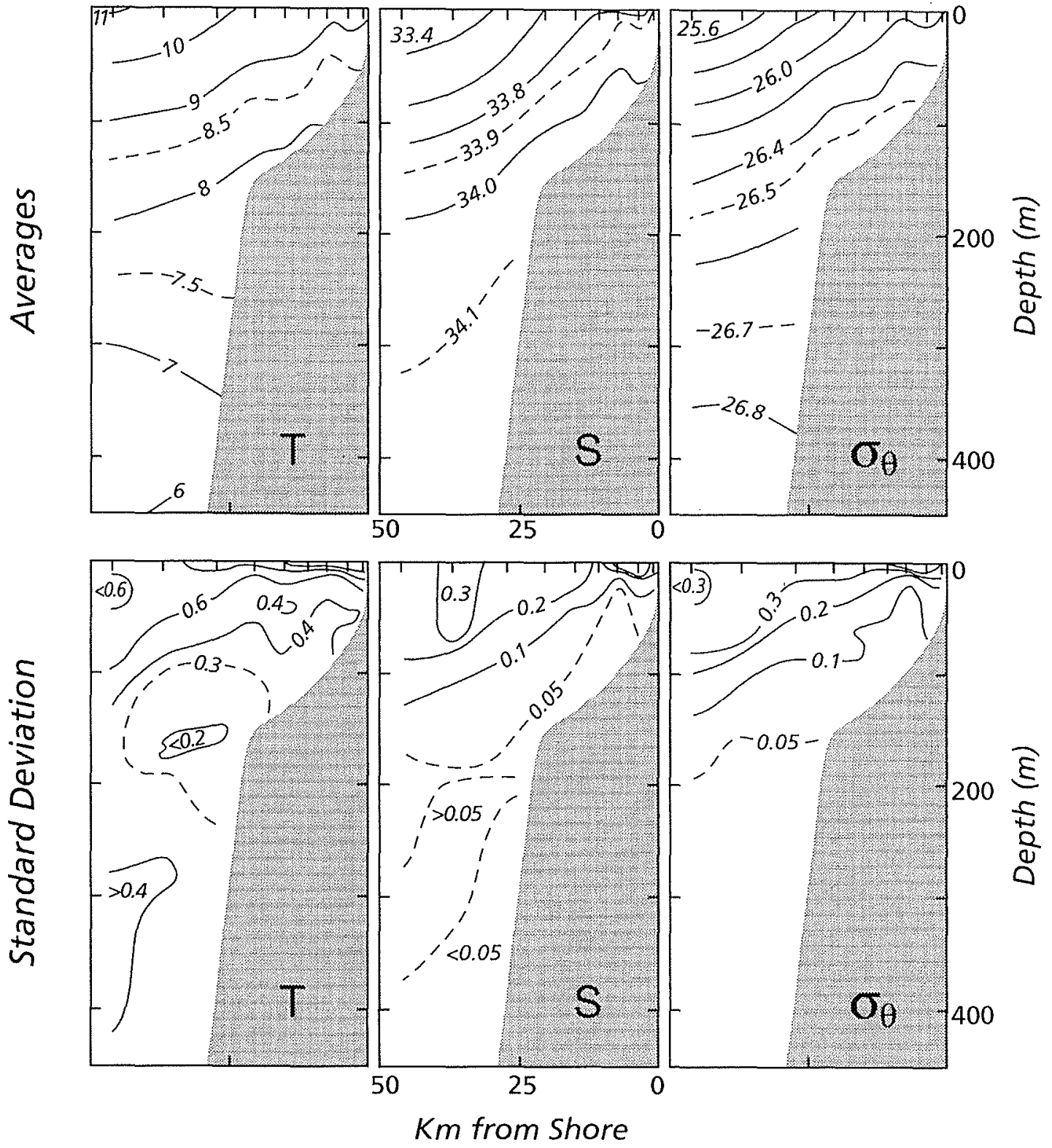

Figure 5.2: The top plots are the hydrography for the CODE region for the AprilJuly upwelling regime, while the bottom plots are the standard deviation. They are taken from Kosro and Huyer [1986]. 


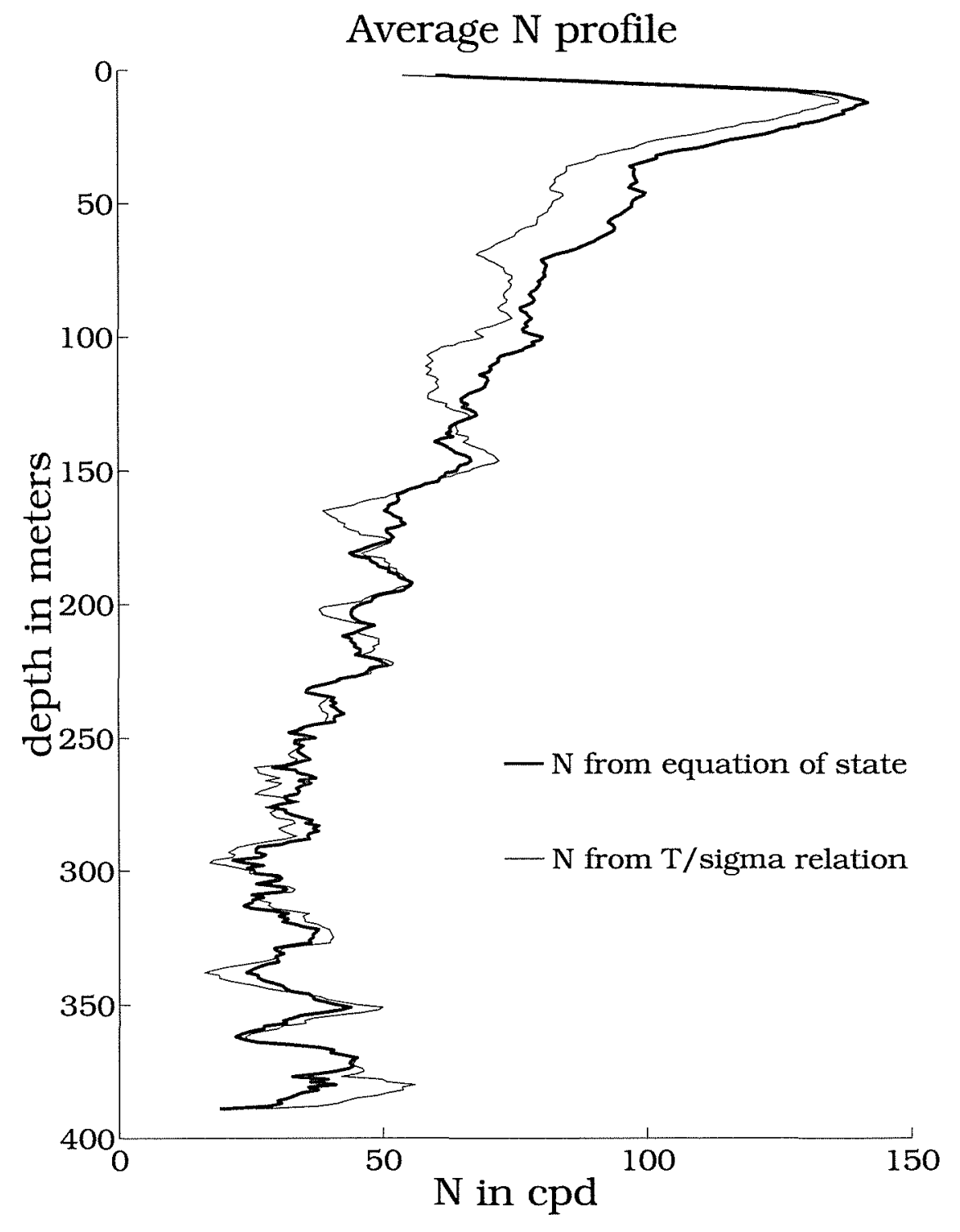

Figure 5.3: A plot of the average buoyancy frequency found from CTD casts on the shelf and shelf break in July of 1982. The thick line is the value computed with a full equation of state, while the thin line uses the empirical relation between temperature and potential density given by (5.5). The data has been smoothed by a ten meter boxcar average. 


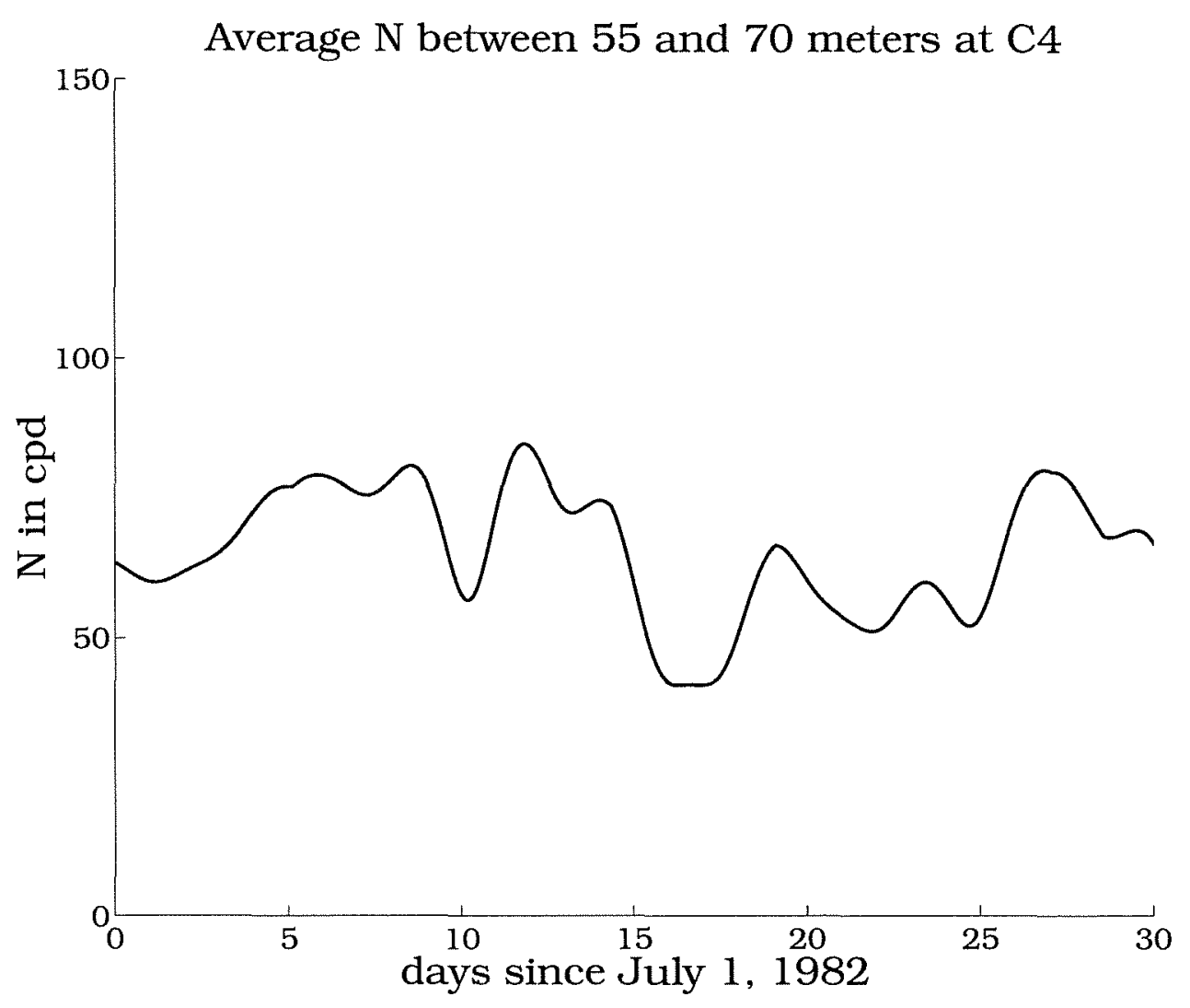

Figure 5.4: The buoyancy frequency of water between 55 and 70 meters at $\mathrm{C} 4$ as a function of time. The data was low-pass filtered with a filter which had a half amplitude pass at 3.25 times the inertial period $(\mathrm{f}=1.24 \mathrm{cpd}$ ), and a full pass at 4 times the inertial period.

one to compute densities from the temperature records at each current meter. A representative mid-depth time series of $N^{2}$ is shown for the water between 55 and 70 meters at the $\mathrm{C} 4$ mooring in figure 5.4. The temperature data used for figure 5.4 were low pass filtered with a half amplitude pass at 3.25 times the inertial period $(\mathrm{f}=1.24 \mathrm{cpd})$, and a full amplitude pass at 4 times the inertial period. This removes the displacements in the density field caused by the internal waves. 


\subsection{Low Frequency Currents}

Unfortunately, the high frequency (7.5 minute) data needed to study internal waves were only archived for the moorings at the 130 and 365 meter isobaths. There are only hourly data available for the moorings shoreward of 130 meters. Thus most of the following analysis can only be done with the $\mathrm{C} 5$ mooring in $365 \mathrm{~m}$ of water, and the $\mathrm{C} 4$ mooring in 130 meters of water. The C4 mooring had usable current meters at 10 and 20 meters on a surface mooring, and at 35, 55, 70, 90 and 121 meters on a subsurface mooring 100 meters away. The C5 mooring had instruments at 20 , 35, 55, 70, 90, 110, 150, 250, and 350 meters [Beardsley, Limeburner and Rosenfeld, $1985]$.

The alongshore sub-inertial currents during July had two main characteristics. At the 90 meter (C3) and 365 meter (C5) moorings alongshore flow was equatorward

$\left(v \approx-5\right.$ to $\left.-15 \mathrm{~cm} \mathrm{~s}^{-1}\right)$ for the first 15 days of the month, and poleward the next 16 days $\left(v \approx 5\right.$ to $15 \mathrm{~cm} \mathrm{~s}^{-1}$ ). This was also true of the alongshore currents at 90 meters and below at the 130 meter (C4) mooring. However, the currents were persistently equatorward in the water above 90 meters at C4. The cross-shelf currents were less vigorous than the alongshore currents. The barotropic current at $\mathrm{C} 4$ is equatorward for the first 15 days, and nearly zero for the next 15 days. Figure 5.5 shows plots of representative alongshore currents at $\mathrm{C} 3, \mathrm{C} 4$, and $\mathrm{C} 5$. For more information on the low frequency currents, see Winant, Beardsley and Davis [1987].

\subsection{Analysis at individual Current Meters}

The GM model makes three strong statements about the internal wave spectrum in the deep ocean. The shape of the power spectrum of horizontal currents goes roughly as $\omega^{-2}$, the energy level is fixed, and the spectrum is isotropic. However, it will be shown below that the shape of the internal wave spectrum on the shelf varies across the shelf, the internal wave energy levels vary with mooring and time, and the spectra are never isotropic.

Figure 5.6 shows spectra from the $\mathrm{C} 5, \mathrm{C} 4$ and $\mathrm{C} 3$ moorings, chosen because they illustrate well the general trends of the spectra. The spectra become less red as one moves onshore, the energy levels decrease, and the current ellipses are not the circles that GM predicts for the deep ocean. (Each of these quantities are plotted separately 
35 meters at $\mathrm{C} 3$

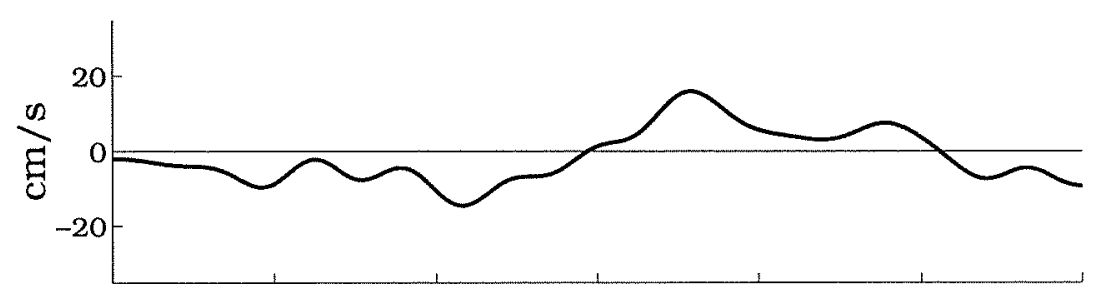

20 meters at $\mathrm{C} 4$
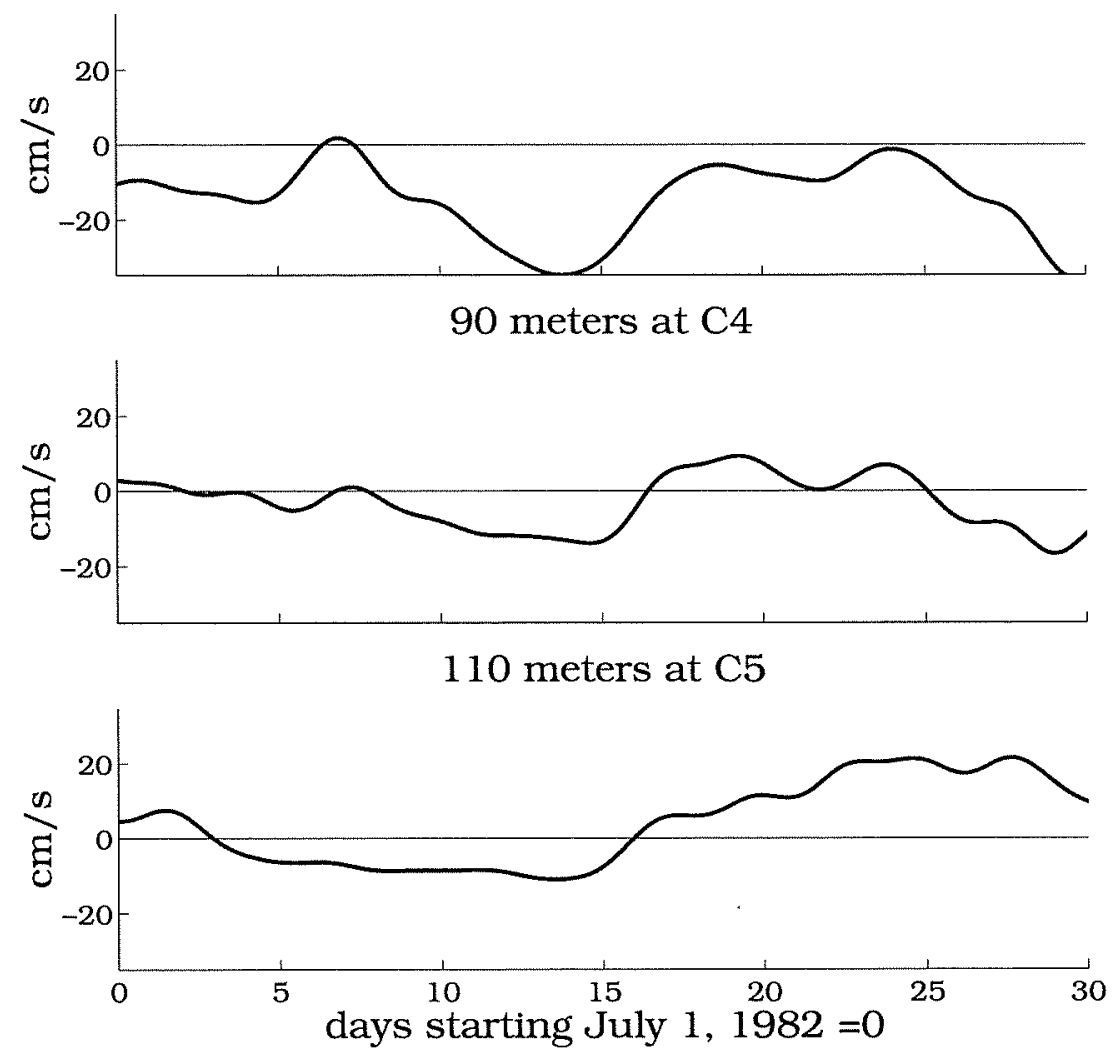

Figure 5.5: Plots of the lowpass filtered along and cross-shore currents. From top to bottom the panels are 35 meters deep at C3, 20 meters deep at C4, 90 meters deep at C4, and 110 meters deep at C5. The same lowpass filter was used as was used in 5.4 . 
latter for all the current meters.) In the section on the modal decomposition of the waves, it will be shown with energy arguments that the high frequency data are consistent with internal waves.

\subsubsection{Power}

The total mean square currents, $\left(u^{2}+v^{2}\right)$, in the 6 to $40 \mathrm{cpd}$ wave band is plotted for each $\mathrm{C} 4$ and $\mathrm{C} 5$ current meter in figure 5.7. The mean square current has been scaled by the GM spectrum for the time averaged buoyancy frequency, so a value of one would match the kinetic energy in the GM spectrum. The power at both locations is consistently larger than the GM power. The power at the C5 mooring is greater than at the $\mathrm{C} 4$ mooring, both when integrated over the water column and averaged over the water column. This reduction of power is consistent in magnitude with the frictional dissipation of shoreward propagating internal waves as described in Brink [1988] and modeled in PB, and it strongly supports the idea that most of the internal wave energy on the shelf is propagating in from the deep ocean or

shelf break and being dissipated as it moves shoreward. If energy were propagating adiabatically from offshore, both the power integrated over the water column and the power per vertical distance should increase as the waves shoal (PB). If the waves were propagating from the coast outward, the power should likewise increase shoreward, with or without friction, because both friction and shoaling work to reduce the energy in the wave as it moves offshore. Thus the reduction of internal wave power as one moves close to the shore is a robust indication that energy is propagating from the deep ocean or shelf break, and is being dissipated as it moves to the shore.

The enhanced energy near the surface and the bottom at $\mathrm{C} 4$ suggests that the internal waves there are dominated by the first baroclinic mode, which is consistent with the modal analysis presented below. The lack of a similar enhancement at C5 argues that mode one is not dominant in the deeper water.

\subsubsection{Spectral Shape}

In figure 5.6, one can see that the $\log / \log$ spectral slope becomes less steep as one moves toward the shore. This bluing of the spectra is confirmed by figure 5.8, which 

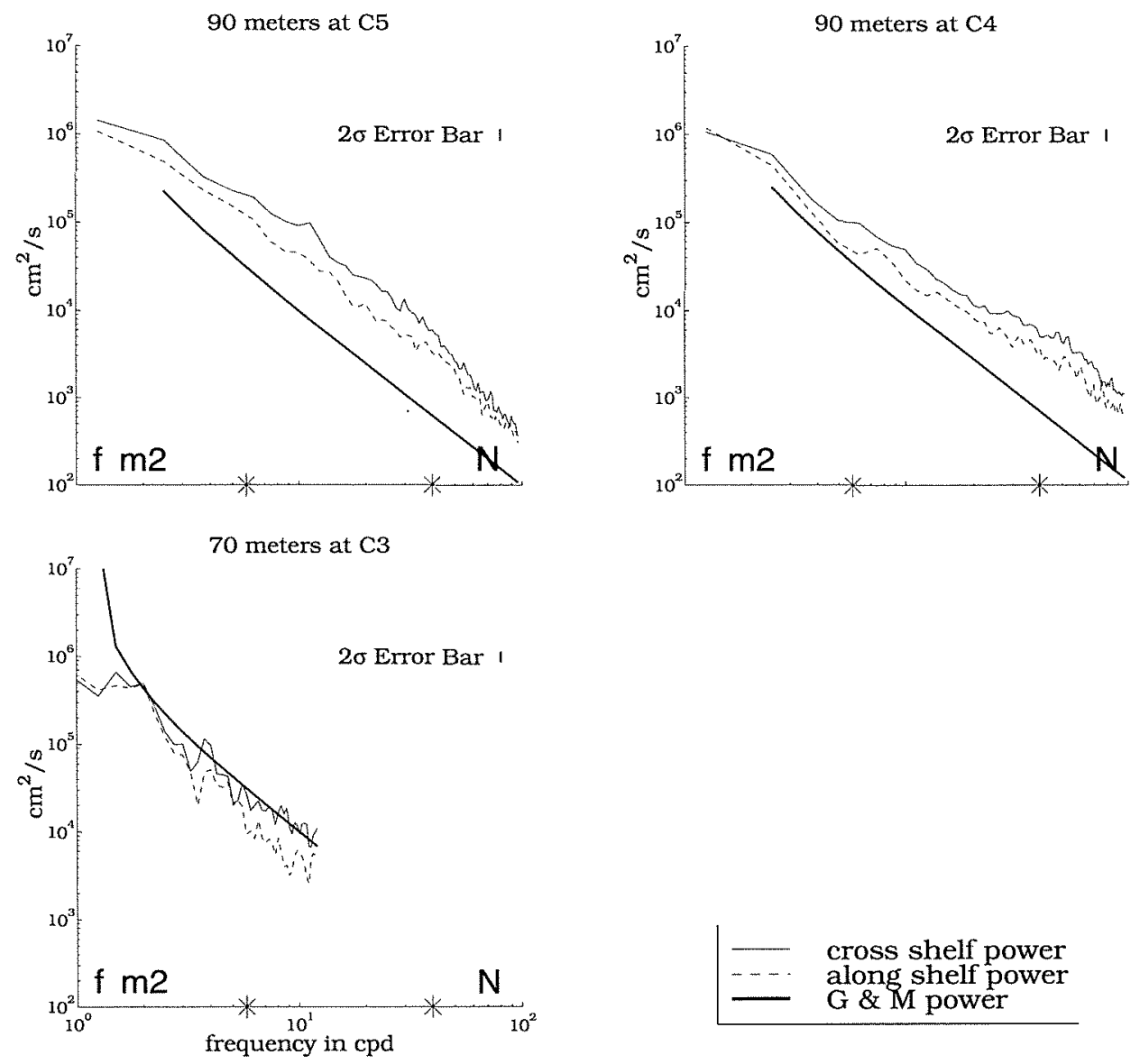

Figure 5.6: The power spectra of cross and along shelf velocity for current meters at 90 meters depth at C5, 90 meters depth at C4, and 70 meters depth at C3. The solid line is the cross-shore power, the dashed the alongshore power, and the thick solid line the GM power. The stars on the frequency axis mark the limits of the frequency range analyzed. The spectrum have units of $\mathrm{cm}^{2} \mathrm{~s}^{-1}$, frequency in cycles per day. 

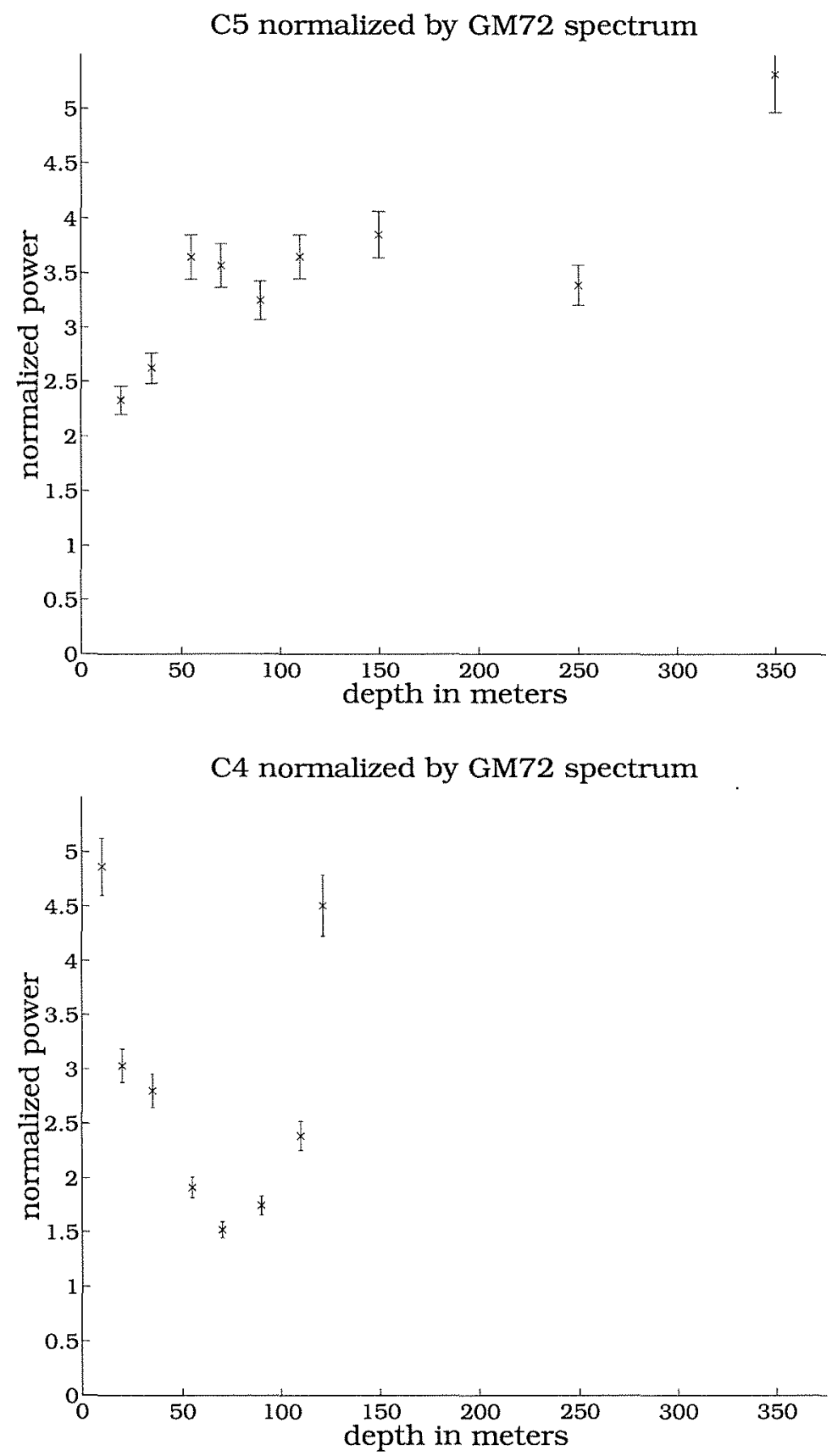

Figure 5.7: Plots of power in the $6-40 \mathrm{cpd}$ frequency band normalized by the GM spectrum. The top plot is for the C5 mooring, the second for C4 mooring. 
shows the spectral slopes at all the current meters on the 365 meter and 130 meter moorings. The slope is computed from a linear least squares fit between the log of the power and the log of the frequencies analyzed. There were not enough spectral data to analyze robustly the power laws at the $\mathrm{C} 3$ site. The slopes at the 365 meter mooring are around 1.8, except for the deepest current meter where the slope is steeper. The slopes for the $130 \mathrm{~m}$ mooring cluster around 1.5. Since the one standard deviation uncertainty of the fits is less then 0.06 , these are significantly different. This bluing of the shallow water spectra is puzzling if one views the internal waves as propagating in from the deep sea. Since the waves decay at a roughly constant rate per unit time, [Brink, 1988; Sanford and Grant, 1987, PB], one would expect the high frequency waves, which are slower, to be dissipated more strongly per unit distance as they move onshore. This would redden the spectrum by selectively dissipating the higher frequency waves. The resolution of this discrepancy may lie in a conjectural non-linear interaction which transfers energy from low frequency waves to higher frequency waves, or in the preferential generation of high frequency internal waves on the shelf or shelf break. This is the greatest disagreement between $\mathrm{PB}$ and the data.

\subsubsection{The Lack of Isotropy- Predictions from PB}

The GM spectrum is isotropic, but it is no surprise that the internal wave spectrum has a strong polarization near the coast. McKee [1973] studied internal waves propagating into a wedge-shaped topography. He noted that internal wave crests turn to parallel the coast as surface gravity waves do. This tends to focus the wave energy toward the coast.

If the internal waves start at the shelf break with no preferred orientation, those that propagate toward the coast turn so that their crests become more parallel to the coast. Since internal waves have current ellipses whose major axes are oriented in the direction the waves are propagating, this turning toward the coast tends to make the current ellipses perpendicular to the local bathymetry.

If the waves are generated near the shore and radiate outward, their orientation depends sensitively on the source location and the orientation of the waves generated at the source. Since current ellipses are symmetric around their major and minor axes, the shape of the ellipse does not indicate whether a wave is coming on or 
C5

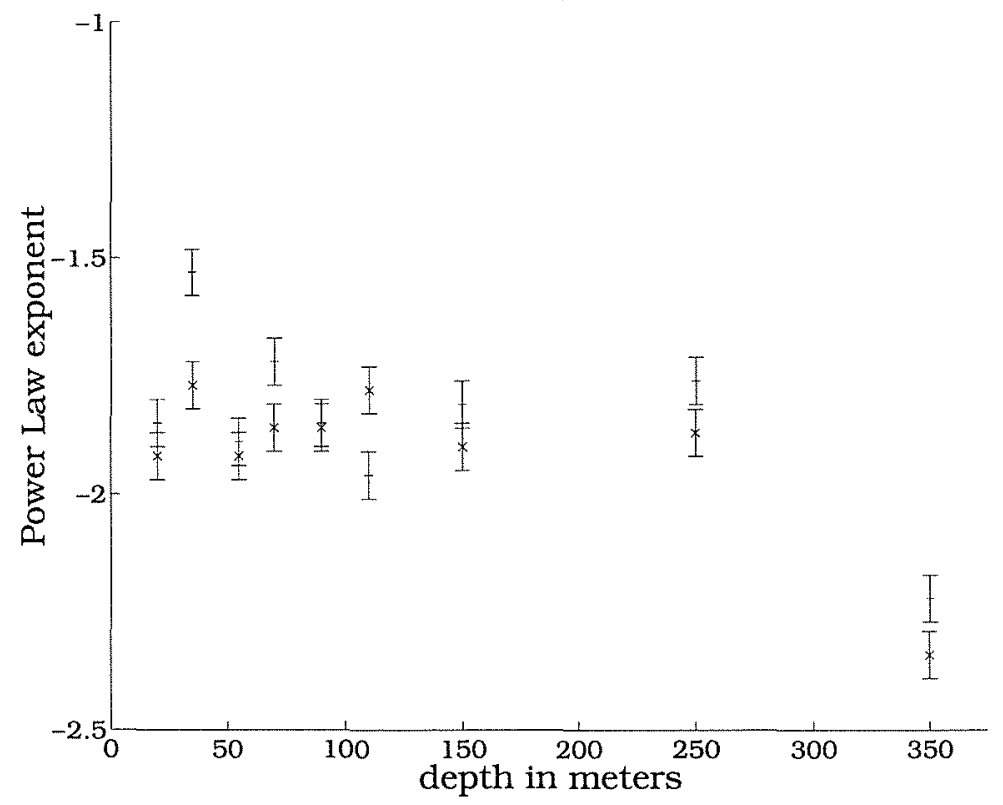

C4

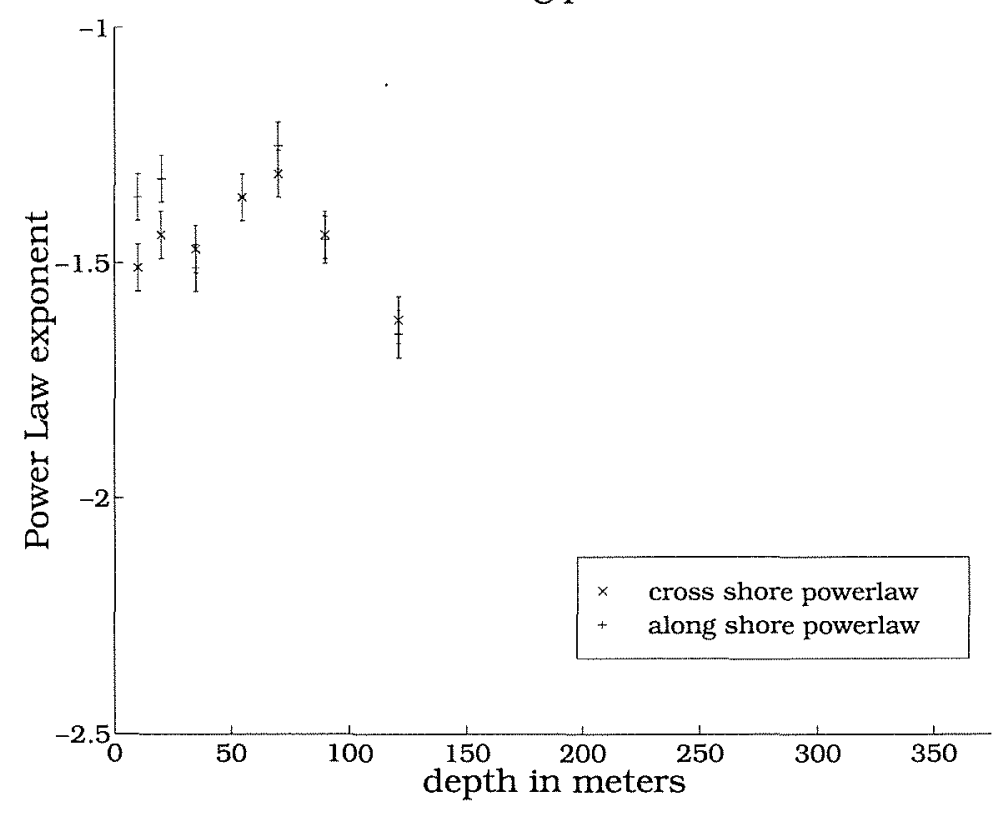

Figure 5.8: Slope of the power spectrum of each current meter in $\log / \log$ space. The top plot is from $\mathrm{C} 5$ and the bottom from $\mathrm{C} 4$. 
offshore. Waves which are generated at the shore but radiate at an angle to the shore will be trapped shoreward of a depth which decreases as the magnitude of the angle to the normal of the shore increases. The ellipse orientation of a trapped wave depends sensitively on the trapping depth and varies as the wave crosses the shelf, and so depends sensitively on the source geometry.

A mean flow can also alter the internal wave geometry. There are many possible effects. Current shear alters the local relative vorticity and hence the apparent $f$ [Kunze, 1985]. The internal wave can trade energy back and forth with the mean current [Lighthill, 1978]. The effect that is found in PB to be dominant at these frequencies and for realistic mean currents is the effect of Doppler shifting a red spectrum. A current will Doppler shift the observed wave, so waves traveling with the mean current will be observed at frequencies higher than their intrinsic frequency, and those traveling against the current will be observed at lower than their intrinsic frequency. If the spectrum of the internal waves in the reference frame of the mean current is red, as it almost certainly is, then the amplitude of the waves traveling with the current, which have lower intrinsic frequencies, will be greater. Because of this, the current ellipse will be shifted in the direction of the current.

All of these effects operate at the same time, so distinguishing the relative contributions of each in the data is hard. PB presents a model of these effects for waves of a GM spectrum at the shelf break propagating over the shelf. The model is a linear ray tracing model which assumes that near the shelf break the path of the internal waves becomes controlled by the bathymetry. This naive treatment of the shelf break, as well as the lack of any alongshore variation in currents and the disregard of any non-linear effects other than wave interaction with a mean flow are the primary weaknesses of the model. However, its simplicity has the advantage that its predictions can be easily summarized:

- Topographic refraction makes the major axis of the current ellipses perpendicular to the isobaths.

- The ellipticity of the current ellipse, which is the ratio of the horizontal current power in the major axis direction to the power in the minor axis direction, will increase closer to shore, but will not depend strongly on wave frequency. Currents of the magnitude seen in the CODE region will not greatly change the ellipticity. 
- The current will tend to shift the major axis of the current ellipse downstream, i.e. for a positive mean current, the angle of the major axis to the crossshore direction is positive (See figure 5.9). For a bathymetry like that in the CODE region, the mean currents will shift the major axis of the current ellipse of vertical mode one and two waves by only 5 to 15 degrees away from the cross-isobath direction for mean currents less than $20 \mathrm{~cm} \mathrm{~s}^{-1}$.

- The effects of Doppler shifting will be stronger for higher vertical modes and higher frequencies, because their wavelengths are shorter.

In order to disentangle the effects of topography and alongshore current in the data, two types of analysis will be performed. First, the ellipticity and orientation of the currents as a function of frequency for each current meter will be analyzed for two time subperiods, one for when the mean currents were predominantly poleward, one for when they were predominantly equatorward. Then, since the predictions of the effects of the alongshore current depend on the vertical mode structure of the wave, a similar analysis will be done on the the modally decomposed data. It is also with this decomposition that the direction of propagation of the waves can be determined, and the power in the vertical and horizontal currents can be compared to internal wave theory.

\subsubsection{Ellipticity}

One observable which indicates anisotropy is the square of the ratio of the major to minor axis of the current ellipse (the "ellipticity"). This is the ratio of the powers in the horizontal currents in a coordinate system aligned with the major axis. For a single plane wave it varies with frequency as $\omega^{2} / f^{2}$. For an isotropic spectrum on a $f$-plane it is, of course, 1 . The theory in PB predicts that the ellipticity of a continuum of waves over the shelf should not be a function of vertical mode, and only a weak function of $\omega$. The basic result of the theory is that as waves propagate in from the shelf break they are confined by topographic refraction to a wedge of angles between $-\theta_{c}$ and $\theta_{c}$. $\theta_{c}$ does not depend on mode or frequency but depends strongly on the water depth. Therefore, even in the high frequency limit where each individual wave's currents are only in the direction of propagation, the net current ellipse of all the waves has the same shape as the wedge of allowable angles. Thus, 
if waves of equal amplitude have been confined to propagate in a direction between $-45^{\circ}$ and $45^{\circ}$ from the offshore direction, the minor axis amplitude should be $\sin \left(45^{\circ}\right)$ of the major axis. Of course, as $\omega$ becomes less and the current ellipse for each wave becomes more circular, the ellipticity will drop, but for the range of frequencies considered here this only affects the ellipticity by $30-40 \%$.

In order to collapse the data at each current meter in each subperiod into a single number, the ellipticity is calculated for each frequency between 6 and $40 \mathrm{cpd}$, and those ellipticities are averaged with a constant weighting, ignoring the differences in energy at each frequency. Since the spectra are red, any power-weighted average is approximately the ellipticity at $6 c p d$, and thus not much more useful than only examining the ellipticity at $6 \mathrm{cpd}$. The averages are therefore made without power weighting. Averaging the ellipticity over a range of frequencies is a sensible thing to do for a random isotropic wave spectrum, for each frequency is an independent sample of the same quantity. Thus the tests presented below for distinguishing the observed wave field from an isotropic random wave field become more robust when the ellipticity is averaged over frequency. However, averaging the observed ellipticity over a range of frequencies is still troublesome, for in the real world the different frequencies are not independent samples of one quantity but are samples of different yet perhaps correlated quantities. However, there is no consistent variation of ellipticity with frequency for various current meters, an observation that agrees with the result in PB that ellipticity should be a weak function of $\omega$.

As an observed quantity, ellipticity is difficult to analyze, for any random time series of currents will have an ellipticity of one or greater. Thus the sample average ellipticity of an observed isotropic spectrum will be greater than one, even though the expected average will asymptote to one as the length of the record increases. However, for this analysis, the ellipticity at a given frequency is different from an isotropic spectrum with $80 \%$ confidence if its ellipticity is greater then 1.37 , and the average ellipticity over the 6 to $40 \mathrm{cpd}$ frequency band considered here is different from an isotropic spectrum at the 95\% level if the ellipticity is greater then 1.37. That both significance levels are 1.37 is a coincidence. These significance levels were found by a Monte Carlo technique: the distributions of ellipticity from $10^{5}$ synthetic isotropic time series was computed, and from this the confidence levels were obtained.

Only one of the 32 estimates of the average ellipticity of the data in table 5.1 fails 


\section{definition of current ellipse angle}
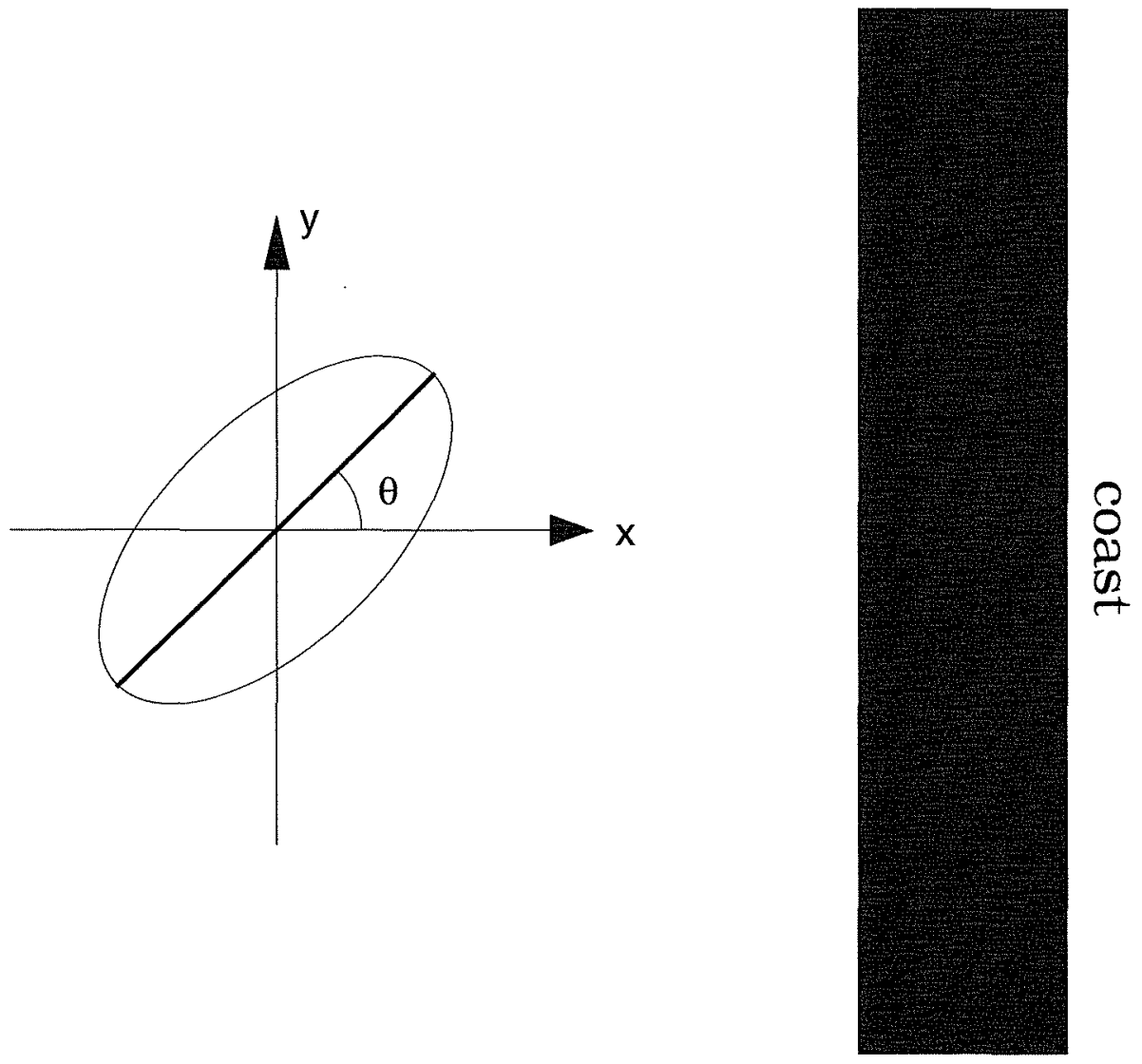

Figure 5.9: The major axis of the illustrated current ellipse is the heavy line, and $\theta$ is the angle of the major axis to the cross shore direction. $\theta$ is positive as drawn. 

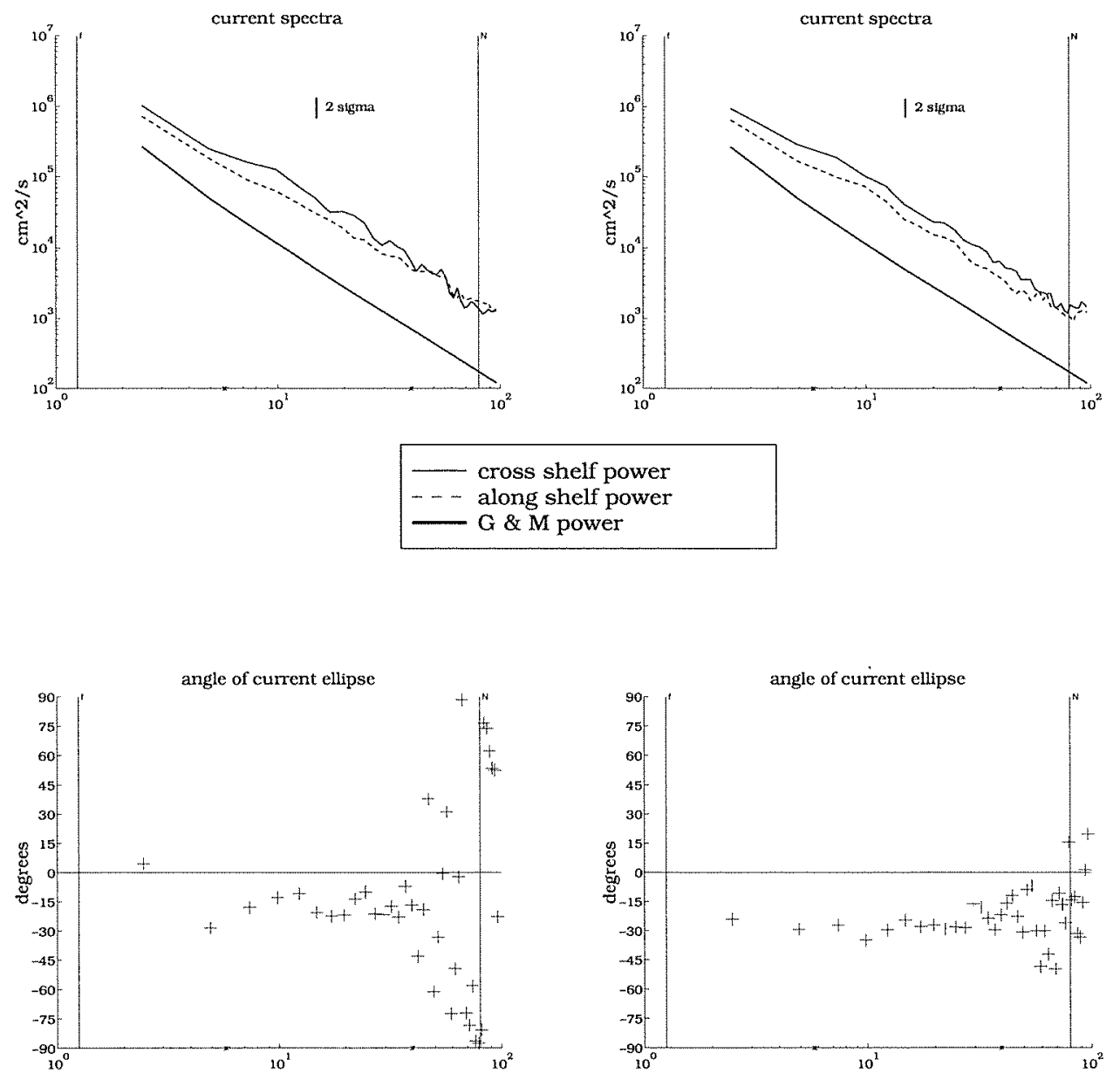

Figure 5.10: These are plots of the power spectra (top) and the angle the major axis of the current meter makes with the cross-shelf direction (bottom) for the first (left) and last (right) half of the July for the current meter in 55 meters of water at the C5 mooring. The stars on the bottom axis mark the frequency limits of the analysis. 
to be different from white noise at $95 \%$ confidence, so the data must be considered anisotropic. The average ellipticities in table 5.1 lie between 2.8 and 1.4 , except for the bottommost current meter at $\mathrm{C} 4$ whose ellipticity is $\approx 4.75$ for the whole record. At C4 the ellipticities are enhanced near the top and bottom to levels that are near the levels that will be obtained for the mode one waves in the modal decomposition below. This is consistent with the idea that the internal wave energy at the $\mathrm{C} 4$ is dominated by mode one, and suggests that this is not true for C5. None of the current records have ellipticities near the $\omega^{2} f^{-2}$ predicted for a single internal wave, so the internal wave field cannot be made up of a single wave propagating in from the shelf break, but is consistent with an ensemble of internal waves propagating across the shelf.

$\mathrm{PB}$ predicts that if all of the internal waves on the shelf had originated as an isotropic wave field in the deep ocean, the ellipticity of the current ellipses should increase as the waves move toward the coast. This is not observed, again suggesting that there is wave generation or scattering on the shelf. Waves generated on the shelf would also tend to be turned toward the coast by the topography and thus become consistent with the PB picture closer to shore. A more detailed comparison to the model in PB is not practical for there is not enough information in the current meter records to compute the horizontal wave number spectra at the current meter moorings, and thus it is impossible to compensate for the naivete of PB's internal wave boundary conditions at the shelf break, or to diagnose what waves are generated on the shelf or at the shelf break between $\mathrm{C} 5$ and $\mathrm{C} 4$.

Interestingly, 13 out of 16 current meters at C5 and C4 had greater ellipticities in the second half of the month, when the water was flowing poleward or less equatorwards. The PB model does not predict this effect, which may be caused by alongshore variation in the mean flow, or localized generation of internal waves on the shelf or shelf break.

\subsubsection{Current Ellipse Orientation}

From the angle of the major axis of the current ellipse to the cross shore direction, one can get a good constraint on the direction of propagation of energy since the current ellipse, horizontal wave vector, and intrinsic group velocity are parallel for a single wave. The mean current will Doppler shift the frequency, but not change the 
before $7 / 15$

after $7 / 15$

\begin{tabular}{|c|c|c|c|c|c|c|}
\hline Mooring & Depth & Mean Angle & ellipticity & sig & Mean Angle & ellipticity \\
\hline & 10 & $-36 \pm 6$ & $2.49 \pm 0.37$ & & $-23 \pm 5$ & $2.79 \pm 0.42$ \\
\hline & 20 & $-72 \pm 7$ & $1.83 \pm 0.39$ & & $-49 \pm 4$ & $2.83 \pm 0.28$ \\
\hline $\mathrm{C} 4$ & 35 & $-16 \pm 53$ & $1.35 \pm 0.21$ & no & $-49 \pm 21$ & $1.60 \pm 0.40$ \\
\hline & 55 & $-22 \pm 9$ & $1.64 \pm 0.25$ & & $-17 \pm 18$ & $1.53 \pm 0.23$ \\
\hline & 70 & $-20 \pm 15$ & $1.83 \pm 0.38$ & & $-2 \pm 9$ & $1.95 \pm 0.36$ \\
\hline & 90 & $-12 \pm 16$ & $1.53 \pm 0.25$ & & $4 \pm 9$ & $2.03 \pm 0.35$ \\
\hline & 121 & $-16 \pm 5$ & $4.41 \pm 1.17$ & & $-16 \pm 4$ & $5.02 \pm 0.80$ \\
\hline & 20 & $-4 \pm 27$ & $1.34 \pm 0.16$ & no & $-15 \pm 15$ & $1.91 \pm 0.34$ \\
\hline & 35 & $-8 \pm 24$ & $1.37 \pm 0.22$ & & $-10 \pm 10$ & $2.00 \pm 0.54$ \\
\hline & 55 & $-17 \pm 5$ & $1.79 \pm 0.31$ & & $-26 \pm 5$ & $2.35 \pm 0.38$ \\
\hline $\mathrm{C} 5$ & 70 & $-9 \pm 9$ & $2.27 \pm 0.47$ & & $-13 \pm 3$ & $2.42 \pm 0.39$ \\
\hline & 90 & $-13 \pm 11$ & $1.93 \pm 0.27$ & & $-9 \pm 6$ & $2.49 \pm 0.31$ \\
\hline & 110 & $-20 \pm 7$ & $2.31 \pm 0.43$ & & $-22 \pm 7$ & $3.21 \pm 0.62$ \\
\hline & 150 & $-12 \pm 6$ & $2.81 \pm 0.69$ & & $-20 \pm 10$ & $2.06 \pm 0.63$ \\
\hline & 250 & $-26 \pm 37$ & $1.53 \pm 0.33$ & & $-34 \pm 21$ & $1.47 \pm 0.31$ \\
\hline & 350 & $-14 \pm 7$ & $2.73 \pm 0.79$ & & $-22 \pm 8$ & $2.62 \pm 0.68$ \\
\hline
\end{tabular}

Table 5.1: The angle that the average current ellipse makes with the cross-shore direction, and the ellipticity of the current ellipse, for the C4 and C5 moorings for the two week periods before and after July 15, 1982. The averages are made by calculating the ellipticity and angle at each frequency in the 6 to $40 \mathrm{cpd}$ frequency range, and then averaging the results with an even weighting. The number after the \pm is the standard deviation of the data, not the expected error in the estimation of the mean. The significance column says no if either the ellipticity or the angle of the current ellipses is not significant at the $95 \%$ level from white noise, using the tests described in the text. 
current ellipse angle, of a single wave. However, the orientation of the major axis is ambiguous to the direction of wave propagation by $180^{\circ}$, so the final determination of the direction of energy propagation must use other methods, such as the evolution of cross-shelf power presented above, or the correlation between velocities presented later.

The orientation of the major axis of the current ellipse is given as the angle the major axis makes to the cross-shore direction. The angle is constrained to be between $-90^{\circ}$ and $90^{\circ}$, and is defined in figure 5.9 as being positive in the counter clockwise sense.

Figure 5.10 includes plots of the ellipse orientation angle for the $55 \mathrm{~m}$ current meter at the C5 mooring. It illustrates a surprisingly constant major axis angle over the 6-40 cpd range of the analysis. The scatter of the angles in figure 5.10 is smaller than in most other records, but none of the other records show a trend in the ellipse orientation with frequency. Since the observed angles show no consistent relation to frequency, they have been averaged over the usual range of frequencies. The averages are in table 5.1. The significance estimate was again formed with a Monte Carlo technique. $10^{5}$ synthetic isotropic random time series were formed, and ellipse orientations as a function of frequency were formed as with the data. The mean angle was defined as the angle which is closest to all the angles in a least squared distance sense. Angular distance was defined as being between $-\pi / 2$ and $\pi / 2$ because ellipse angle is ambiguous to a $\pi$ radian rotation. The standard deviation of angles around this mean was found for each synthetic isotropic series, and a distribution of these standard deviations was found. The standard deviation of the angles in the data was then compared to the distributions of the synthetic data. All but one of the current meter records have angles whose distributions are significantly ( $>95 \%$ confidence) different from an isotropic wave field. This again shows that the wave field must be considered anisotropic

Several conclusions can be drawn from the angles in table 5.1. 31 of 32 averaged angles are negative, and many are near the $-17^{\circ}$ value consistent with bathymetric steering and thus are consistent with linear internal wave energy propagating to the shore. (The shelf break is rotated relative to the shore by $-17^{\circ}$.) The angles at $\mathrm{C} 4$ are more negative when the bottom half of the water column flows to the equator, as the Doppler shift theory in PB would predict. Conversely, the angles at C5 are less negative when the currents are flowing equatorward. This is puzzling, for it is not 
what $\mathrm{PB}$ would predict, and the difference is in the sign of the effect, not just the magnitude. Perhaps some alongshore variability in the currents is focusing internal waves. There is one other puzzling thing in the table: the $20 \mathrm{~m}$ current meter at $\mathrm{C} 4$ has current ellipses with angles of $\approx-60^{\circ}$ to the cross-shelf direction with very little scatter with frequency. It is unclear what could cause this.

\subsection{Modal Decomposition}

From a modal decomposition of the data, the direction of wave propagation can be found, the vertical structure of the waves can be found, and more detailed comparisons can be made to the PB theory. The power in the horizontal and vertical currents can also be shown to be consistent with linearized internal waves. Unfortunately, decomposition into vertical modes can only be done at $\mathrm{C} 4$, for the current meters at C5 are badly spaced for a decomposition, and the hourly data at the shallower site make it difficult to compute robust statistics. The details of the decomposition have been left to the appendix, but a summary follows. Using the mean buoyancy profile for the month, figure 5.11, the vertical modal structures are calculated for a frequency of $10 \mathrm{cpd}$, so $f^{2} \ll \omega^{2} \ll N^{2}$. These modes, also illustrated in figure 5.11, are shown in PB not to vary substantially with frequency for frequencies greater than the critical frequency of reflection and lower than the buoyancy frequency. It is these restrictions which define the 6 to $40 \mathrm{cpd}$ frequency range to which the gathering of statistics is confined. The modes are fit at each time step to the current meter data, with $u, v$, and $w$ fit independently. The fit is optimal in a least squares sense. $w$ is determined from the time derivative of temperature and the vertical gradients of temperature.

For this decomposition, only the first two modes are estimated with confidence. These two modes account for $71 \%$ of the cross-shore current variance, $50 \%$ of the alongshore variance, and $66 \%$ of the vertical velocity variance in the frequency range analyzed. Table 5.2 breaks this down by variable and mode. The angle of the current ellipses and the ellipticity of the current ellipse for the mode 1 wave are shown in figure 5.12 .

The ratio of the power in the horizontal currents to that in the vertical currents for a given mode can be used to check if the high frequency data are consistent with internal waves. The ratio of the horizontal current power to the vertical current 


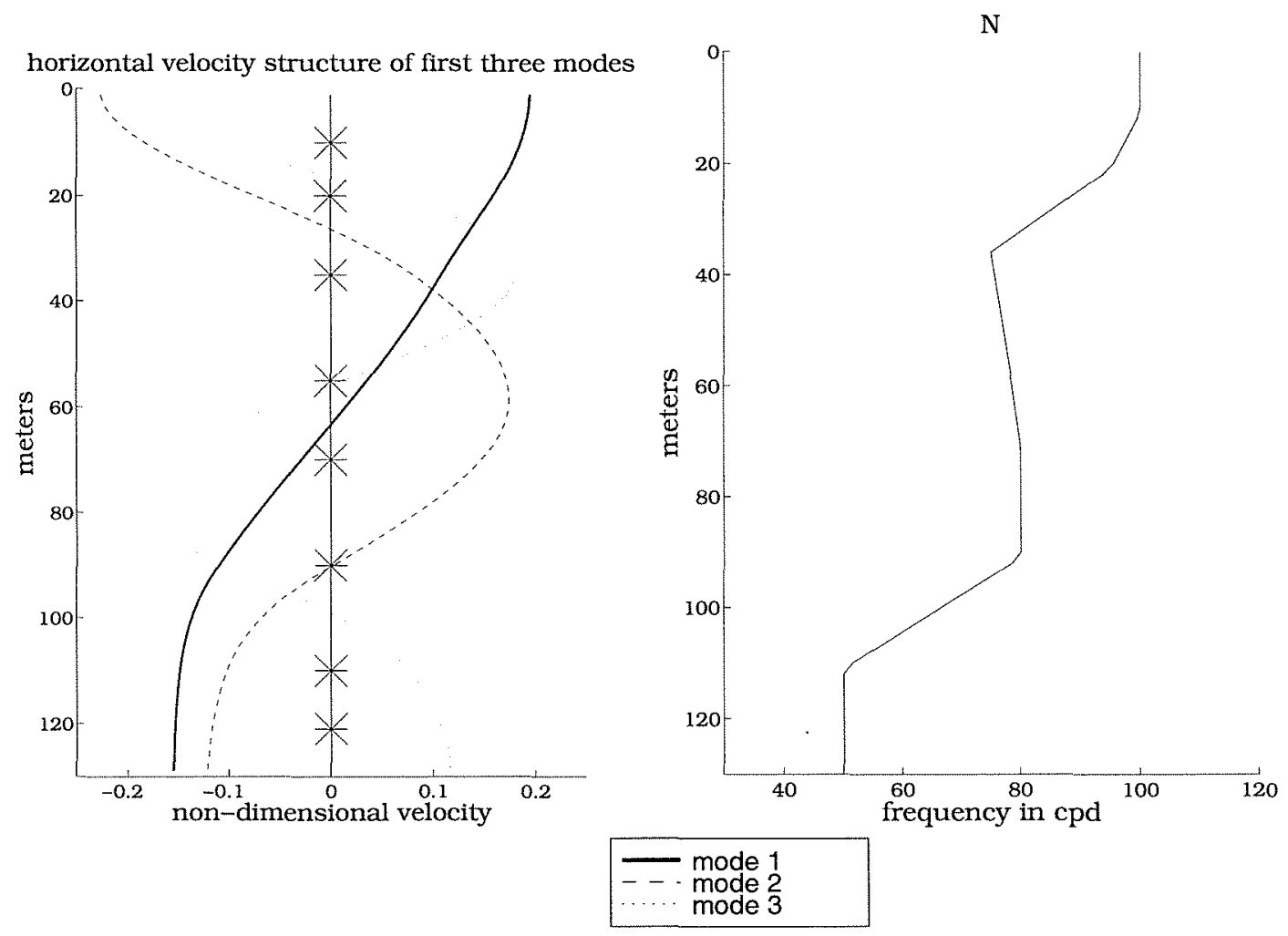

Figure 5.11: The left hand panel is a plot of the horizontal velocity structure of the first three modes, while the right hand plot is a plot of the buoyancy frequency profile. The stars on the vertical axis mark the position of the current meters at $\mathrm{C} 4$. 

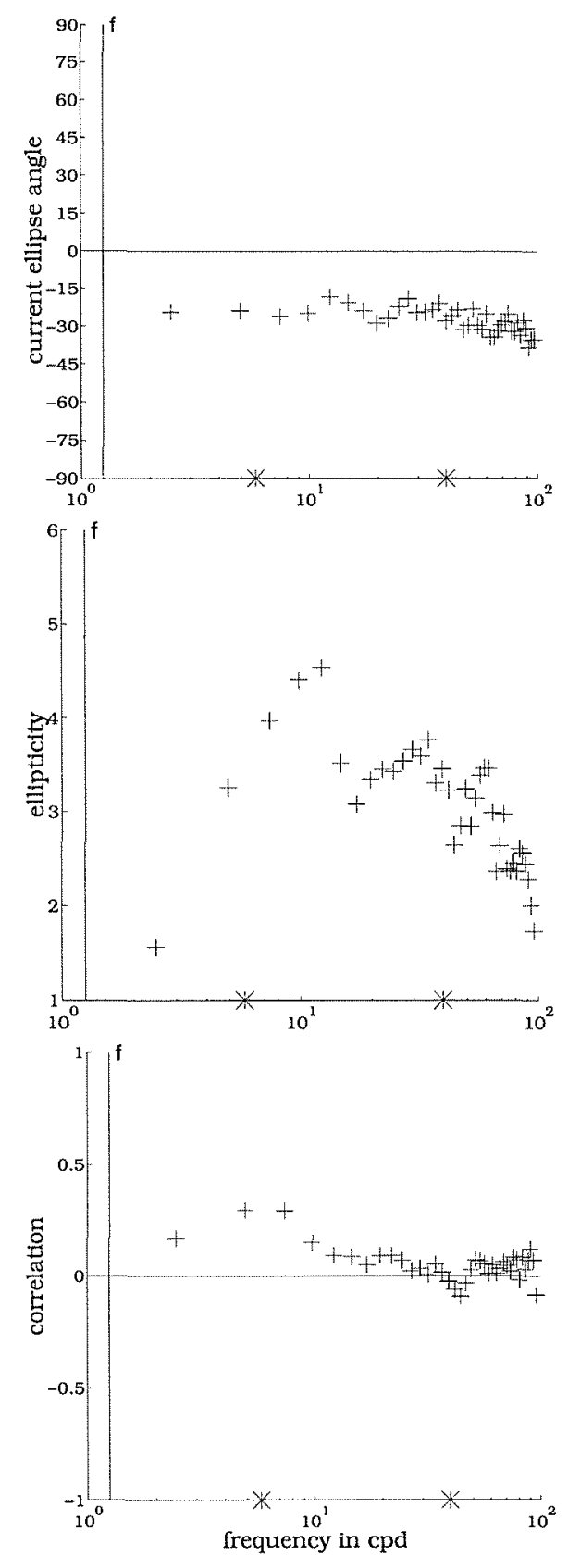

Figure 5.12: Plots of the angle of the current ellipses (top), the ellipticity of the current ellipse (middle), and the correlation between the currents parallel to the minor axis of the ellipse and the vertical velocity (bottom) for mode 1 . The stars on the horizontal axis mark the frequency limit of analysis. 


$\begin{array}{clll}\text { Mode } & \text { Alongshore velocity } & \text { Cross-shore velocity } & \text { Vertical velocity } \\ 1 & 35 \% & 53 \% & 53 \% \\ 2 & 15 \% & 17 \% & 15 \% \\ \text { 3/Barotropic } & 11 \% & 4 \% & 10 \% \\ \text { Residual } & 32 \% & 27 \% & 22 \% \\ \text { Total Variance } & 94 \% & 101 \% & 100 \%\end{array}$

Table 5.2: The percent variance explained by mode for the modal decomposition. The third mode for the horizontal velocities is the barotropic mode, not the real third mode. It is fit to reduce the crossing of any barotropic mode into the first and second modes. The total variance does not sum to $100 \%$ because the discretely sampled modes are not orthogonal. Details are in the appendix.

power for a given mode should be [Fofonoff, 1969, PB]

$$
\frac{\left\langle v^{2}\right\rangle+\left\langle u^{2}\right\rangle}{\left\langle w^{2}\right\rangle}=\frac{N^{2}-\omega_{r}^{2}}{\omega_{r}^{2}-f^{2}}\left(1+f^{2} \omega_{r}^{-2}\right),
$$

for an ocean with a constant $N$, where $\omega_{r}$ is the frequency of the wave in a frame of reference moving with the barotropic water velocity. $\mathrm{PB}$ demonstrates that replacing the constant $N$ with a depth averaged $N$ makes little difference if $\omega_{r}^{2} \ll N_{\min }^{2}$, where $N_{\text {min }}$ is the smallest $N$ in the water column, in this case about $50 \mathrm{cpd} . \omega_{r}$ differs from $\omega$ by $(\mathrm{PB})$

$$
\omega_{r}=\omega-\sin (\theta) V \frac{M \pi}{D} c
$$

where $V$ is the alongshelf barotropic current, $M$ the mode number, $D$ the water depth, $\theta$ the orientation of the internal wave to the cross-shelf direction, and $c$ is the characteristic slope of an internal wave, from (5.3). $V$ varies with time, and at any given frequency there is an ensemble of waves with varying orientations and amplitudes. In figure 5.13, the results for modes one and two are plotted with the curves predicted by (5.6) and (5.7) for $V= \pm 10 \mathrm{~cm} \mathrm{~s}^{-1}$ and $\theta= \pm 24^{\circ}$, the latter being the current ellipse angle for mode one, described below. The data agree well with the curves from (5.6), especially with the curve in which $\theta$ and $V$ are oriented in the same direction, as they are in the data.

The most robust result of the decomposition is that the first mode is dominant at $\mathrm{C} 4$. In the cross-shelf velocity, the first mode explains $53 \%$ of the variance, the second $17 \%$. In the alongshelf, the numbers are $35 \%$ for the first mode, $15 \%$ for 

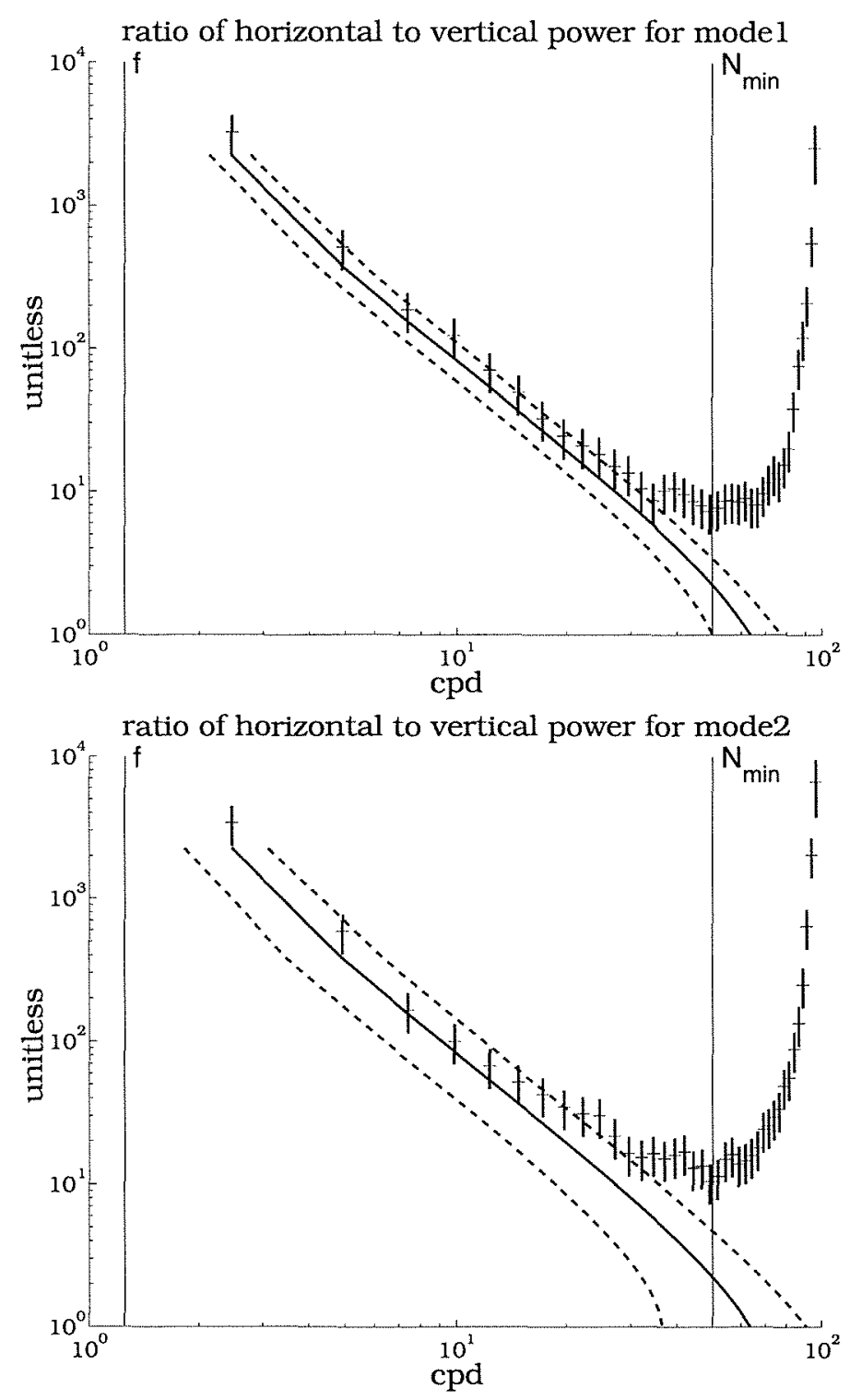

Figure 5.13: The ratio of the horizontal and vertical current powers for mode 1, top, and mode 2 , bottom. The solid line is the solution to (5.6) when $V=0$ and/or $\theta=0$, while the dashed lines are for $V= \pm 10 \mathrm{~cm} \mathrm{~s}^{-1}$ and $\theta= \pm 24^{\circ}$. The lower curve is for when the sign of $V$ and $\theta$ differ, the upper for when they are of the same sign. The error bars on the data are \pm one standard deviation. 
the second, and in the vertical velocity, $53 \%$ in the first mode, $15 \%$ in the second. The lower power in the first mode alongshore currents compared to the cross-shelf and vertical velocities suggests that waves trapped to the coast are less dominated by the first mode. The higher power in mode one is consistent with Brink [1988], which predicts that modal internal waves decay at a constant rate per time. Thus, if the waves are coming from the shelf break, those which travel faster will be the least dissipated when they reach a mooring on the shelf. Since second mode waves have group velocities half as large as first mode waves, they ought to be, and appear to be, more attenuated than first mode waves. Of course, there may have simply been less mode 2 energy entering the shelf from the deep ocean or generated at the shelf break. A more detailed comparison between model and theory is thwarted by a lack of knowledge of the relative strength of the modes at the shelf break.

The orientation of the current ellipses is nearly cross-isobath, though the orientation changes somewhat with time and mean currents. The depth mean current is equatorward for the first 15 days of July, and nearly zero subsequently [Winant, Beardsley and Davis, 1987]. Nevertheless, the first mode angle is hardly changed, remaining about $-24^{\circ}$ as the current changes, while the slower second mode changes its angle from $-33^{\circ}$ when the bottom current is equatorward to $-13^{\circ}$ when the current is poleward. This $20^{\circ}$ change in angle is comparable to the $10^{\circ}$ swing predicted by $\mathrm{PB}$ for a $10 \mathrm{~cm} / \mathrm{s}$ barotropic current and an isotropic GM spectrum at a $400 \mathrm{~m}$ shelf break. In the end, however, the magnitude of the predicted effect of mean current on angle is small, and the uncertainties in the predictions and the data analysis are large. Thus while the second mode data are not inconsistent with the Doppler shift model in PB, nor are the data inconsistent with the simpler model that ignores currents and says that the waves will be perpendicular to the local bathymetry and propagating towards the shore.

PB predicts that the internal wave field propagating in from the shelf break will be focused into a narrow range of angles, and thus the ellipticity of the currents will become independent of frequency and mode. Unfortunately, the predictions of what the ellipticity will be in $\mathrm{PB}$ are sensitive to even small amounts of wave energy generated on the shelf. Any waves generated on the shelf can reduce the ellipticity by an order of magnitude if the maximum offshore extent of those waves coincides with the position of the mooring, even if the energy generated on the shelf is much less than the incoming internal waves. The ellipticity of the observed mode 
one waves is about 3.6 while that of the second mode is 2.3 , with neither showing an obvious frequency dependence. This suggests there is relatively more alongshore propagating energy in the second mode than in the first. Also, for both modes, the ellipticity increases in the second half of the month, though only in the first mode is this increase significant, from 3.29 to 4.34 . This suggests a decrease in alongshore energy in the second part of the month. The tentativeness of this analysis indicates the difficulty of understanding the internal wave field without some measure of the horizontal wave number spectrum.

The modal data can help to determine the direction of internal wave propagation. To do this, it is necessary to define the "spectral correlation coefficient." This is the normalized cospectrum of two time series at a given frequency, defined as

$$
\langle v w\rangle=\frac{C o_{v w}}{\sqrt{P_{v v} P_{w w}}},
$$

where $C_{o_{w}}$ is the cospectrum of $v$ and $w$, and $P_{v v}$ and $P_{w w}$ are the power at a given frequency in $v$ and $w$ respectively. The structure of an internal wave propagating in the $x$ direction is, when represented modally

$$
\begin{aligned}
& u=-\frac{1}{k} \frac{d W}{d z} \sin (k x-\omega t+\phi), \\
& v=\frac{f}{k \omega} \frac{d W}{d z} \cos (k x-\omega t+\phi),
\end{aligned}
$$

and

$$
w=W(z) \cos (k x-\omega t+\phi)
$$

where $k$ is the wave number, $\omega$ the angular frequency, and $\phi$ is a phase (PB). From (5.9a) it is easy to see that for a single plane wave traveling in the positive $x$ direction, the correlation $\langle v w\rangle$ is one, and the major axis of the internal wave is oriented along the $x$ axis. Thus, for a single wave, once the angle of the major axis of the current ellipse has been determined for a given frequency, the mean direction of wave propagation along the major axis can be determined from the sign of the correlation of the vertical velocity and the current in the minor axis direction. Unfortunately, for a set of internal waves propagating onto the coast with a range of angles, the computation of the correlation is more complicated. As a simplified version the the model in $\mathrm{PB}$, the correlation is calculated assuming waves of equal 
amplitude and random phase propagating at angles from $-\theta_{c}$ to $\theta_{c}$ around the major axis. Computing the theoretical correlation of the minor axis velocities and the vertical velocities for modally decomposed waves of random phase and constant amplitude, one obtains

$$
\langle v w\rangle=\frac{\frac{2 f}{\omega} \sin \left(\theta_{c}\right)}{\sqrt{2 \theta_{c}\left(1+\frac{f^{2}}{\omega^{2}}\right)+\theta_{c} \sin \left(2 \theta_{c}\right)\left(\frac{f^{2}}{\omega^{2}}-1\right)}},
$$

where $\theta_{c}$ is computed in $\mathrm{PB}$ as a function of $\delta$, the ratio of the water depth at the observation site to the depth where an internal wave first "feels" the bottom:

$$
\theta_{c}=\arctan \left(\frac{1}{\sqrt{\delta^{-1}-1}}\right)
$$

$\theta_{c}$ is shown as a function of $\delta$, and $\langle v w\rangle$ as a function of $\theta_{c}$, in figure 5.14. Assuming $\delta \approx 0.3$ to 0.4 for the 130 meter site, the correlation should lie between 0.1 and 0.4 . Correlations of 0.1 to 0.4 for a single frequency are hard to distinguish from white noise. However, it becomes possible to test for propagation onshore if the test is whether the correlation is positive. This test distinguishes waves going onshore from a random wave field, whose correlation would be randomly distributed around zero, and also from waves propagating offshore. It cannot test between various models of the distribution of energy with angle. The test is made by rotating the data at each frequency so that $u$ is in the direction of the major axis that points onshore, and $v$ is parallel to the minor axis which makes the coordinate system righthanded. Then the correlation $\langle v w\rangle$ is formed, and plotted in figure 5.12. Table 5.3 gives the percentage of frequencies whose correlation is positive and the confidence that this percentage is distinguishable from an isotropic internal wave field. Since $u$ is rotated so that the onshore component of a positive $u$ is positive, when $\langle v w\rangle$ is positive the waves are propagating onshore. All of the mode one data are consistent with onshore propagation at a better than $93 \%$ level, while the mode 2 data are only consistent with onshore propagation before July 15 . The mode 2 correlations after July 15 are indistinguishable from white noise.

There are some caveats to this method. The correlation is very sensitive to the range of angles over which the waves are propagating. Thus, if an ensemble of waves propagated onshore at a range of angles from $-10^{\circ}$ to $10^{\circ}$ and the same amount of energy went offshore spread between $-30^{\circ}$ and $30^{\circ}$, this method would indicate that 

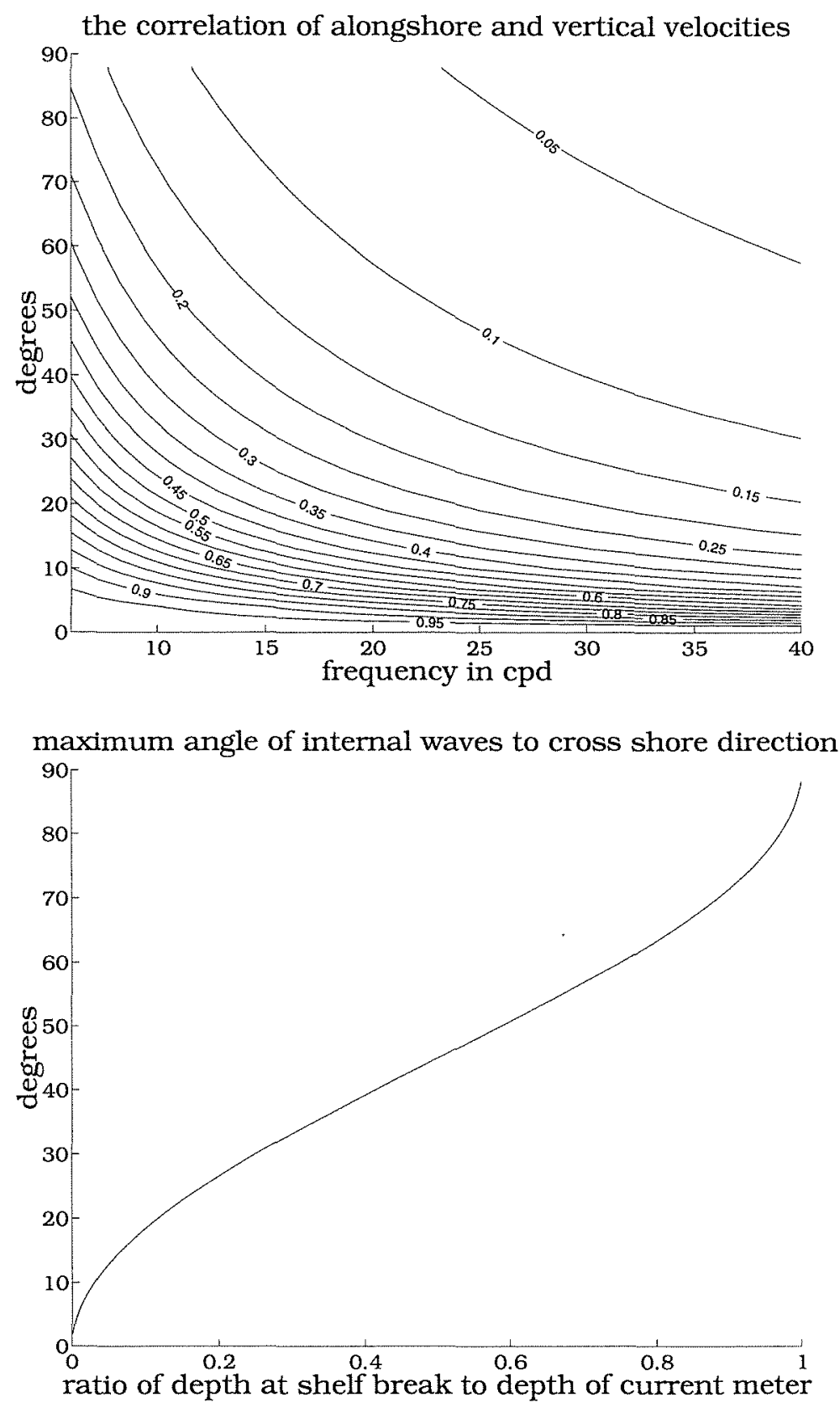

Figure 5.14: The top panel is a plot of the correlation between the vertical velocity and the horizontal velocity parallel to the minor axis as a function of angular beam width and wave frequency. The bottom panel plot is the beam width as a function of the ratio of depth at which the waves feel the bottom to the depth of the current meter. 


$\begin{array}{rcccc} & \text { Mode 1 } & \text { Confidence } & \text { Mode 2 } & \text { Confidence } \\ \text { before July 15 } & 100 \% & >95 \% & 71 \% & 93 \% \\ \text { after July 15 } & 71 \% & 93 \% & 45 \% & \text { none }\end{array}$

Table 5.3: The percentages are the percentages of frequency bins whose vertical velocity versus minor axis velocity correlation is greater than zero. For waves traveling onshore, this should be $100 \%$, for a isotropic wave field it should be $50 \%$. The confidence level is the confidence that it is the former, not the later.

energy was propagating into the shore. These are limitations of not measuring the wave number spectrum directly.

\subsection{Variation in Power with Space and Time}

Inherent in the GM spectrum is an assumption that the energy levels of the internal wave spectrum are constant with time. This is not true for the internal waves on the shelf in the CODE region. The determination that the energy levels are not stationary is tricky, for any finite length moving average of a truly random time series will fluctuate. These fluctuations are larger for a red spectrum. Figure 5.15 is a plot of a two day moving average of the power over the 6 to $40 \mathrm{cpd}$ frequency band from the 35, 70, 90 and 121 meter current meters at $\mathrm{C} 4$, along with one standard deviation error bars computed from the periodograms. The error bars account for the redness of the spectrum. The mean energy is also plotted. The standard deviations of the data are small enough, and the degrees of freedom large enough, that the chi square distributions can be considered Gaussian. The observations from 35 meters were more than one standard deviation from the month-long mean $32 \%$ of the time, at 70 meters $68 \%$ of the time, at 90 meters $67 \%$ of the time, and at 121 meters $80 \%$ of the time. For a stationary Gaussian distributed time series, this should only be true $34 \%$ of the time, indicating that the power levels are not stationary.

Neither the fluctuations in $N$ plotted in figure 5.4 or the fluctuations in the alongshore wind in figure 5.16 show any correlation with the power fluctuations. This is also true for the time series of $N$ at the other depths, the time series of winds at other nearby locations, and the time series of alongshore current and current shear. 
current meter at $35 \mathrm{~m}$

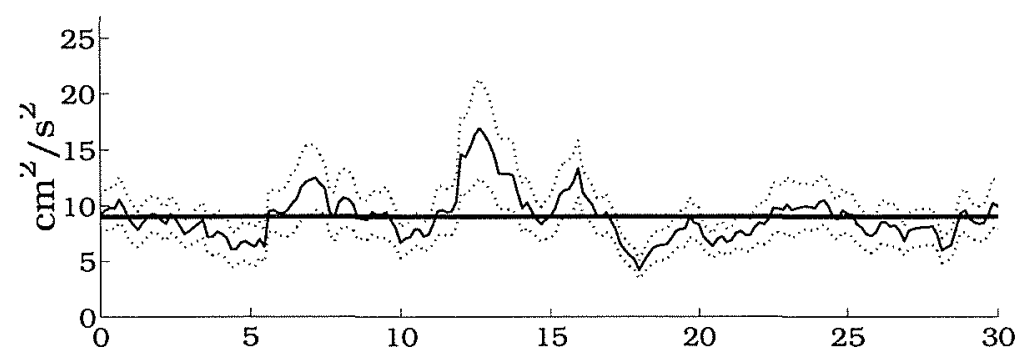

current meter at $70 \mathrm{~m}$

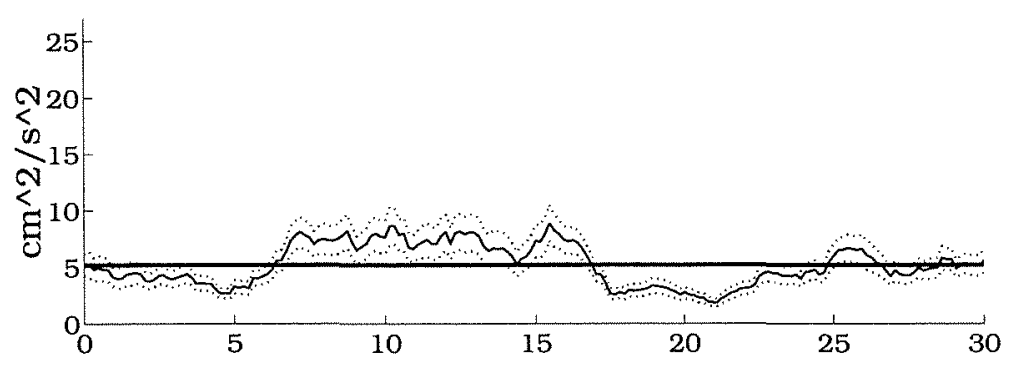

current meter at $90 \mathrm{~m}$

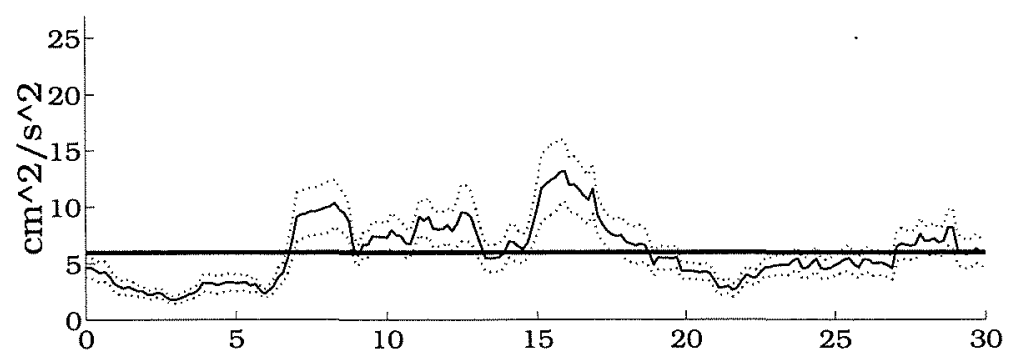

current meter at $121 \mathrm{~m}$

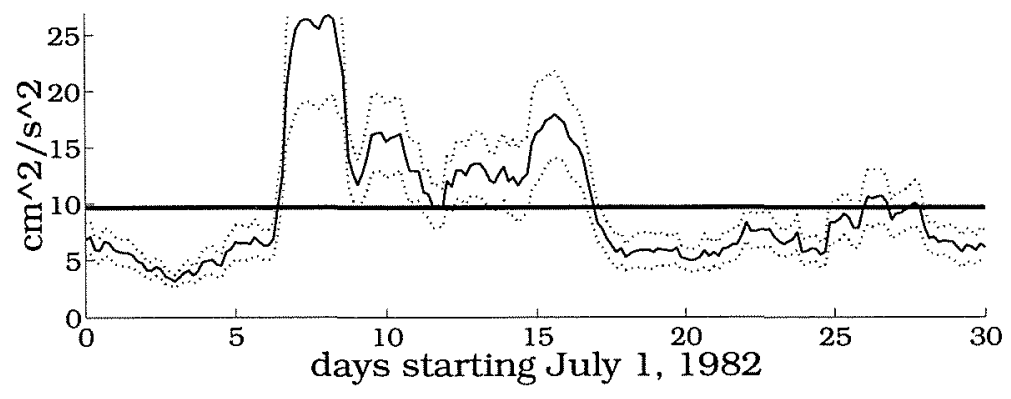

Figure 5.15: Plots of a two day moving average of the power from the 35, 70,90 and 121 meter current meters at the 130 meter site, along with one standard deviation error bars. The mean power is plotted as the heavy horizontal line. 


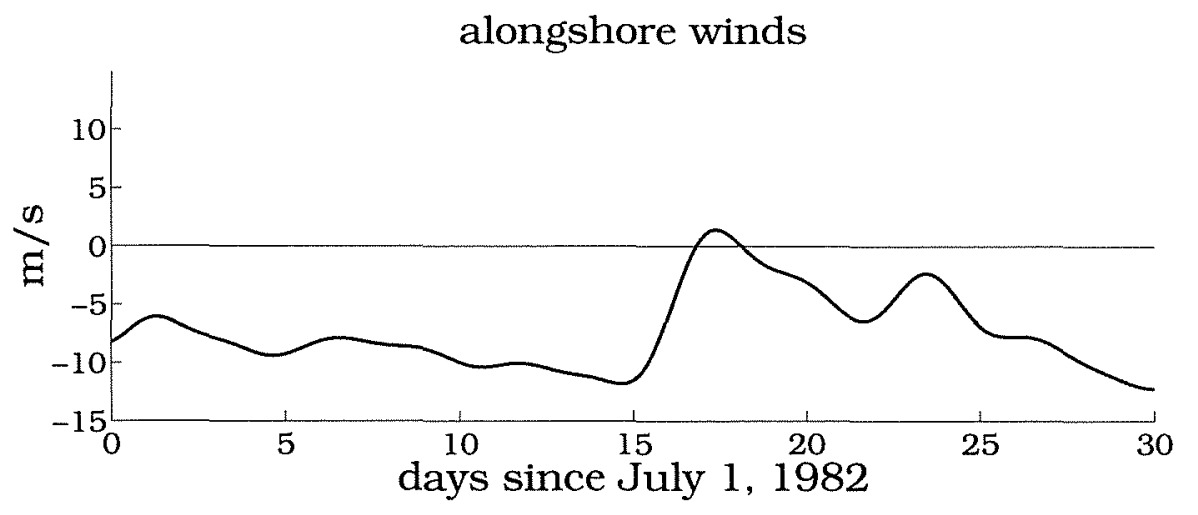

Figure 5.16: This is a plot of the along shelf winds at $\mathrm{C} 4$ for the month of July. The winds have been lowpassed filtered with the same filter as used for figures 5.4 and 5.5 .

Perhaps the fluctuations in internal wave power are the results of the focusing of internal waves by alongshore variations in the current field.

\subsection{Discussion and Conclusion}

The observations can be summarized by contrasting those results that are consistent with internal wave energy existing in the deep ocean as a GM spectrum and propagating onto the shelf, and those results that suggest that waves must also be generated, or at least severely modified from the predictions of a linear theory, on the shelf.

The decline of total energy levels approaching the shore strongly supports the model of waves originating in deep water or at the shelf break and being eroded by friction as they propagate onshore. In a scenario with no friction, or any scenario for generation of outward propagating waves near the shore, the energy levels would be expected to increase near the coast.

The orientation and ellipticity of the current ellipses is consistent with an ensemble of internal waves propagating in from the deep ocean, though the failure of the ellipticity to increase shoreward (as predicted by PB) suggests that waves generated on the shelf are also propagating onshore, thus broadening the angular distribution of the waves. Propagation toward the coast is also supported by the sign of the 
correlation of the vertical and minor axis velocities of mode one and two waves.

The dominance of mode 1 waves at the $\mathrm{C} 4$ site is again consistent with the frictional model in $\mathrm{PB}$, since mode 1 waves are the least affected by friction. This argument cannot be made more convincing, however, without a better knowledge of how deep water modes penetrate onto the shelf. It seems that mode 1 does not dominate at $\mathrm{C} 5$.

There are facts that conflict with the picture in PB and indicate that while a GM spectrum propagating in from the deep ocean or shelf break is probably the dominant source of internal waves on the shelf, it is certainly not the only one. It may be that a significant portion of the internal wave energy on the shelf is generated on the shelf. It may also be that the theory is too naive in its linearity, in its simple assumption that the alongshore current is barotropic, or in its assumption of no alongshore variability in topography on frequency .

The most puzzling finding is that the spectrum becomes less red closer to shore. The slower high frequency waves should be dissipated more rapidly than the low frequency waves. The observed bluing of the spectrum means that either energy is being generated preferentially at higher frequencies on the shelf, or that non-linear interactions are shifting the energy in the spectrum to the higher frequencies.

Thus the most plausible qualitative synthesis of observations is of a GM spectrum propagating onto the shelf accompanied by shoreward propagating waves generated on the shelf. In order to clarify this possibility it would be necessary to have observations of the modal structure of the waves at several different locations, and ideally some knowledge of the directional spectrum of the waves.

It is also worth observing that on continental shelves broader than at the CODE region, $\mathrm{PB}$ indicates that it is likely that dissipation would eliminate all waves from the deep ocean before the shore is reached, which would considerably alter the internal wave climate.

\subsection{Acknowledgments}

I would like to thank the people who helped me get the data for this paper, especially Susan Tarbell and Carol Alessi, both of whom helped me find the current meter data and tutored me in the interpretation of mooring logs. The data came from the CODE programs and so thanks go to all who collected the data and planed the 
programs. This paper benefited greatly from Steve Lentz's close reading, and would have been impossible without Ken Brink's many readings and numerous pieces of scientific advice. The work was funded by an Office of Naval Research fellowship and and Office of Naval Research AASERT fellowship, N00014-95-1-0746.

\subsection{Appendix: The Modal Decomposition}

In a flat bottom ocean, the internal wave spectrum can be broken into vertical modes which are orthogonal to each other (e.g. LeBlond and Mysak [1978]). Given $u$ and $v$ as horizontal velocities, and $w$ as the vertical velocity, the linearized system of equations on an $f$-plane is

$$
\begin{aligned}
\frac{\partial u}{\partial t}-f v & =-\frac{1}{\rho_{0}} \frac{\partial P}{\partial x}, \\
\frac{\partial v}{\partial t}+f u & =-\frac{1}{\rho_{0}} \frac{\partial P}{\partial y}, \\
\frac{\partial w}{\partial t} & =-\frac{1}{\rho_{0}} \frac{\partial P}{\partial z}-\frac{\rho g}{\rho_{0}}, \\
\frac{\partial u}{\partial x}+\frac{\partial v}{\partial y}+\frac{\partial w}{\partial z} & =0
\end{aligned}
$$

and

$$
\frac{\partial \rho}{\partial t}-\frac{\rho_{0}}{g} N^{2}(z) w=0 .
$$

This system admits internal wave solutions of the form:

$$
\begin{aligned}
& u=-\frac{1}{k} \frac{d W}{d z} \sin (k x-\omega t+\phi), \\
& v=\frac{f}{k \omega} \frac{d W}{d z} \cos (k x-\omega t+\phi),
\end{aligned}
$$

and

$$
w=W(z) \cos (k x-\omega t+\phi),
$$

where $\phi$ is a phase, $k$ the horizontal wave number, $\omega$ the angular frequency, and $W(z)$ the vertical modal structure. $W(z)$ is determined by

$$
\frac{d^{2} W}{d z^{2}}+k^{2}\left(\frac{N^{2}(z)-\omega^{2}}{\omega^{2}-f^{2}}\right) W=0
$$


and

$$
W=0 \text { at the top and bottom. }
$$

If $N$ is constant, $W$ has the form

$$
W=\sin \left(\frac{M \pi}{D} z\right) \quad M=0 \ldots \infty
$$

If $N$ depends on depth, the structure of the mode is no longer independent of $\omega^{2}$. However, as shown in PB, if $N^{2} \gg \omega^{2}$, as is true of this analysis, the modal structure is independent of $\omega^{2}$ to a very good approximation. Thus the modal decomposition will be done for the $\omega=10 \mathrm{cpd}$ modes. The results are insensitive to the choice of $\omega$.

This analysis is only true for a flat bottom, however, since the boundary condition is $W=0$ at the bottom. However, Wunsch [1969] showed that the vertical modal solution is approximately valid for waves whose frequencies are much less than the frequency of critical reflection, which is true for this analysis. This approximation is justified in greater detail in PB.

The actual decomposition of the data was done on the horizontal velocities and vertical velocities. The vertical velocities were estimated by assuming the temperature balance

$$
T_{t}+w T_{z}=0
$$

and computing $T_{z}$ from low pass filtered current meter data. The modal functions were computed for the mean hydrography. This is less critical than it may appear, for the low modes are not very sensitive to changes in $N$. The amplitude of each mode was found by fitting the computed modes to the current meter data at each time record, fitting $u, v$, and " $w$ " independently. The data were depth weighted, but this made little difference because the current meters were nearly evenly spaced. The fit was optimal in a least squares sense.

This straightforward method obscures several subtle issues in the decomposition. The first problem is that, while the internal wave modes are orthogonal, a set of discrete measurements in the water column are not in general orthogonal. Unfortunately, to speak meaningfully of power in a mode, that mode must be orthogonal to all others. In order that all of the modes that are fit are 
nearly orthogonal to each other (in the sense that for two modes $W_{1}$ and $W_{2}$, $W_{1} \cdot W_{2} / \sqrt{\left(W_{1} \cdot W_{1}\right)\left(W_{2} \cdot W_{2}\right)} \ll 1$ where $W_{1} \cdot W_{2}$ is the inner product of $W_{1}$ and $W_{2}$ evaluated at the measurement points), only 5 modes can be fit to the seven current meters. The fit is judged successful because the sum of the power in each mode and the residual (as shown in table 5.2) is never more than 6\% different from the total power. If the data from the top two current meters are discarded, the power in the modes explains $180 \%$ of the variance, with most of the duplication of power being in the lowest two modes. Thus discarding the top two current meters is untenable.

It is unfortunate that the need for orthogonality forces one to use the top two current meters, since they are on a different mooring. This mooring is described as nominally being one hundred meters to the northwest of the subsurface mooring, and was certainly not more than $200 \mathrm{~m}$ away [Beardsley, Limeburner and Rosenfeld, 1985, and S. J. Lentz, pers. com.]. The horizontal decorrelation length scale for horizontal currents will be half a wavelength for waves with a finite beam width.

Figure 5.17 is a plot of the wavelengths of the first 5 modes as a function of frequency. From this it can be seen that if the separation between the first two moorings is $100 \mathrm{~m}$, mode 2 becomes nearly decorrelated at 40 cycles per day. In the worst case, with a separation of $200 \mathrm{~m}$, the second mode becomes decorrelated at about 23 cycles per day.

The amount of power in a real mode that is spuriously transfered to another mode because of any decorrelation of the top two meters from the lower current meters can be calculated. This calculation shows that, at the worst case of $200 \mathrm{~m}$ separation, $20 \%$ of mode 1 horizontal current power will leak into mode 2 , while 20 $\%$ of the horizontal current power in mode 2 can leak into mode 1 . However, only $5 \%$ percent of the vertical current power in mode 2 can leak into mode 1 vertical current power and vice versa. Little mode 1 and 2 power would be leaked to the higher modes. The vertical power is $53 \%$ in the first mode and $15 \%$ in the second mode, and the cross-shore power is $53 \%$ in the first mode and $17 \%$ in the second (table 5.2). This partition remains true for frequencies in the 25-40 cpd range. If the two moorings were so far apart that mode 1 would decorrelate, the cross-shore mode 2 power would be greater than the vertical mode 2 power, since more of the horizontal mode 1 power would leak into horizontal mode 2 than vertical mode 1 power into vertical mode 2. Likewise, if only mode 2 decorrelated, the fraction of cross-shore power in mode 2 would be less than the fraction of vertical power in 


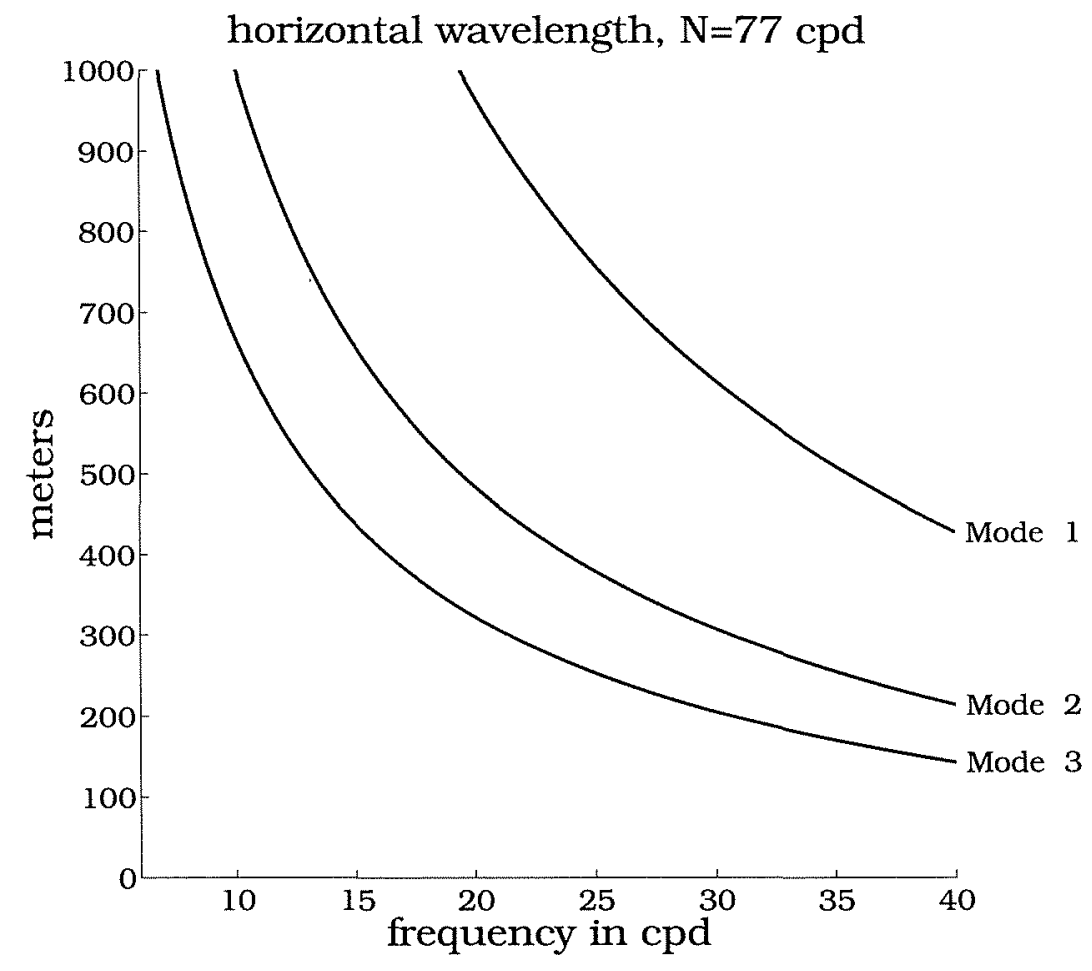

Figure 5.17: This is a plot of the wavelength of the first three internal wave modes in 130 meters of water as a function of frequency. 
mode 2 since more mode 2 power would leak in the horizontal modes than vertical modes. Thus the the moorings are probably $100 \mathrm{~m}$ or less apart.

Even with only a hundred meters between the two current meter moorings, it must be assumed that modes higher than the second are decorrelated. Similar calculations to the ones above show that it becomes impossible to sort out the energy in the higher but resolvable modes, but that the partition of about $30 \%$ of the energy to modes higher than the second mode is robust.

The possibility of modes higher than the theoretically observable fifth mode being aliased into the low modes is impossible to rule out, but seems improbable because the energy decreases monotonically for the first five modes. 


\subsection{Bibliography}

Beardsley, R. C. and S. J. Lentz, The Coastal Ocean Dynamics Experiment Collection: An Introduction, J. Geophys. Res., 92(C2), 1455-1463, 1987.

Beardsley, R. C., R. Limeburner and L. K. Rosenfeld, Introduction to CODE-2 Moored Array and Large-Scale Data Report in CODE-2: Moored Array and Large-Scale Data Report, Woods Hole Oceanographic Institute, Technical Report 85-35, Nov. 1985.

Brink, K. H., On the effect of bottom friction on internal waves, Continental Shelf Research, 8(4), 397-403, 1988.

Fofonoff, N. P., Spectral Characteristics of Internal Waves in the Ocean, Deep Sea Res., 16, 59-71, 1969.

Garrett, C. and W. Munk, Space-Time Scales of Internal Waves, Geophys. Fluid Dyn., 3, 225-264, 1972.

Gordon, R. L., Internal wave climate near the coast of northwest Africa during JOINT-1, Deep Sea Res., 25, 625-643, 1978.

Howell, T. L. and W. S. Brown, Nonlinear Internal Waves on the California Continental Shelf, J. Geophys. Res., 90(C4), 7256-7264, 1985.

Huyer, A., J. Fleischbein and R. Schramm, Hydrographic Data From The Second Coastal Ocean Dynamics Experiment: R/V Wecoma, Leg 9, 6-27 July 1982, College of Oceanography, Oregon State University, Data Report 109, Ref. 84-7, Apr., 1983.

Kosro, P. M. and A. Huyer, CTD and Velocity Surveys of Seaward Jets Off Northern California, July 1981 and 1982, J. Geophys. Res., 91(C6), 7680-7690, 1986.

Kunze, E., Near-Inertial Wave Propagation in Geostrophic Shear, J. Phys. Oceanogr., $15,544-565,1985$.

LeBlond, P. H. and L. A. Mysak, Waves in the Ocean, Elsevier Scientific Publishing Co, Amsterdam, 1978.

Lighthill, J., Waves in Fluids, Cambridge University Press, Cambridge, 1978. 
Lynch, J. F., G. Jin, R. Pawlowicz, D. Ray, C. -S. Chiu, J. H. Miller, R. H. Bourke, A. R. Parsons and R. Muench, Acoustic travel-time perturbations due to shallow-water internal waves and internal tides in the Barents Sea Polar Front: Theory and experiment, Journal of the Acoustical Society of America, 99(2), 289-297, 1996.

McKee, W. D., Internal-inertial Waves in a Fluid of Variable Depth, Proc. Camb. Phil. Soc., 73, 205-213, 1973.

Sandstrom, H. and J. A. Elliott, Internal Tide and Solitons on the Scotian Shelf: A Nutrient Pumps at Work, J. Geophys. Res., 89(C4), 6415-6426, 1984.

Sanford, L. P. and W. D. Grant, Dissipation of Internal Wave Energy in the Bottom Boundary Layer on the Continental Shelf, J. Geophys. Res., 92(C2), 1828$1844,1987$.

Winant, C. D., R. C. Beardsley and R. E. Davis, Moored Wind, Temperature, And Current Observations Made During Coastal Ocean Dynamics Experiments 1 and 2 Over the Northern California Continental Shelf and Upper Slope, J. Geophys. Res., 92(C2), 1569-1604, 1987.

Wunsch, C., Geographical Variability of the Internal Wave Feild; a Search for Sources and Sinks, J. Phys. Oceanogr., 6, 471-485, 1976.

Wunsch, C., On the propagation of internal waves up a slope, Deep Sea Res., 15, $251-258,1968$.

Wunsch, C., Progressive internal waves on slopes, J. Fluid Mech., 35, 131-144, 1969. 


\section{Chapter 6}

\section{Conclusion}

The preceding chapters are only the beginnings of what need to be studied in order to understand the effect of winter time cooling on the coastal ocean and the internal wave climate in the coastal ocean. This conclusion section presents what I think would be the most useful next steps to understanding these phenomena better.

\subsection{Winter Time Cooling and the Coastal Ocean}

The second chapter describes the transition between a flow dominated by the vertical mixing driven by surface cooling and a turbulent geostrophic flow. In order to understand the transition, the vertical mixing driven by convective overturning was parameterized with a scheme based, like Mellor and Yamada [1982], on laboratory results which neglect rotation and background horizontal density gradients. This is troubling and hard to justify a priori. It should be, however, just within the grasp

of modern computers to resolve all scales from convective to geostrophic arrest in one computer simulation and directly simulate the transition from vertically convecting flows to nearly geostrophic turbulent flows. This would be an ambitious project. Intellectually it spans the space from geostrophic turbulence to convection. Computa- 
tionally it would require a non-hydrostatic simulation capable of resolving convective scales, i.e. horizontal scales of order the water depth, yet with a domain large enough to contain geostrophic turbulence, i.e. a domain size of the same order or larger than the relevant turbulence arrest scale. Even with a turbulence submodel to span the range of scales from sub-convective to Kolmogorov, this implies a computational task similar in size to a large global ocean general circulation model, which makes it just within the reach of a graduate student starting a thesis in the late 1990's.

The third chapter uses simple models of geostrophic turbulence to scale for the eddy flux of heat across-the shelf. These scalings assume that the velocity anomaly of the geostrophic turbulence scales as the product of the mean thermal wind shear and the water depth. It is difficult to justify this rigorously, though it is a common assumption [Stone, 1972; Visbeck, Marshall and Jones, 1996]. Attempts to find a better scale for the velocity multiply the thermal wind velocity scale by a function of the Burger number [Held and Larichev, 1995]. Extending the work in chapter 3 to include scales for the stratification would allow one to use these more sophisticated scales for the velocity anomaly, and perhaps explain why the product of the length of the instabilities and the velocity anomaly are predicted more accurately by the scalings than either quantity alone.

Chapter 3 also ignores wind forcing, which can both change the eddies which move heat offshore and drive flows which transport heat offshore. An alongshore wind will set up ageostrophic cross-shelf flows in the surface and bottom boundary layers. These flows would shear the top and bottoms of the eddies, and it would be useful to understand how and if this affects the eddies. The wind-forced boundary layer flows will also transport heat on- or offshore and, in so doing, alter the depth averaged stratification which controls the static and baroclinic stability of the fluid. It would thus be useful to understand both the wind driven heat flux and the wind's effect on the mean stratification. 
It is also assumed in the above work that temperature is the sole determinant of density. There is, however, a mean cross-shelf salinity gradient over most continental shelves created by riverine runoff, and this salinity gradient tends to make the near shore waters less dense than the offshore waters. Further, as the temperature of the water falls, the importance of the salinity in the equation of state of seawater increases $[$ Gill, 1982]. In the Nantucket Shoals Flux Experiment the density field at the end of the 1979-1980 winter was temperature-dominated to the 100 meter isobath, at which point the density increased offshore due to the salinity gradient (figure 1.1, Beardsley et al. [1985]). It is an open theoretical question whether an eddy could propagate through a region where the cross-shelf density gradient reverses, and it is further unclear whether a mixing formulation as given in chapter 3 would be appropriate in such a case. This question is further complicated on the Mid-Atlantic Bight by the existence of the shelf break front nearby.

The shelf break and the shelf break front further complicate applying chapter 3 to the actual ocean by making it difficult to estimate the heat flux from the deep ocean onto the shelf. It is assumed in chapter 3 that this heat flux is known, but it is even unclear to what extent the heat transport across the shelf break is set by the water properties on the shelf, meteorological events, or external forcing from the deep ocean (such as warm core rings).

Despite these uncertainties, chapters two and three begin to explain how an icefree coastal ocean responds to winter time cooling. It is found that the strong vertical mixing driven by cooling need not always mix the entire water column, and when it does not cross-shelf eddy fluxes of heat can be sufficient to balance surface cooling. This mechanism is likely to be important in establishing the hydrography of a winter time coastal ocean. 


\subsection{Observations and Theory of High Frequency Internal Waves over the Continental Shelf}

The study of internal waves in the coastal ocean can be advanced most rapidly by new observations. The non-linear interactions between internal waves on the shelf, the mechanisms of generation of internal waves on the shelf, and the response of internal waves on the shelf to vertically sheared flows are all important areas of theoretical interest. Nonetheless, the almost complete lack of knowledge of the evolution of the horizontal wave number spectrum of internal waves crossing the shelf makes it hard to know which of the outstanding theoretical questions to concentrate on.

Given a horizontal wave number spectrum of internal waves at a locations on the shelf, one could trace the waves back past their origin, though the location of the point of generation along the ray path would be unknown. By observing the evolution of the spectrum across the shelf, the location of wave generation on the shelf can be pinned down. This would give valuable information about the non-linear processes and generation mechanisms of the internal waves created on the coast.

Observations of the horizontal wave number spectrum would also help to define the internal wave flux across the shelf break. Chapter four assumes that the internal wave spectrum at the shelf break can be modeled as a Garrett and Munk spectrum, despite the explicit disavowal in Garrett and Munk [1972] of any applicability of the spectrum near horizontal or vertical boundaries. Since understanding what the spectrum should be at the shelf break requires understanding what sets the Garrett and Munk internal wave spectrum, and since this has not yet been done convincingly, observations may be the best way to proceed.

The horizontal wave number spectrum is a difficult quantity to observe because of the broad temporal and spatial bandwidth of the internal waves over the shelf. Because spatially broad band processes have decorrelation length scales of the order 
of the wavelength being observed, any observation of the spatial spectrum would need to be done with an instrument or instruments capable of taking measurements spaced much less than a wavelength apart. Making the task somewhat easier, waves of small spatial scale have high frequencies, and can thus be prevented from contaminating the measurements with temporal filters.

The difficulty of this measurement task can be seen by estimating the horizontal wavelength of a vertical mode one internal wave, $\lambda_{h}$

$$
\lambda_{h} \approx \frac{N D}{2 \omega},
$$

where $N$ is the buoyancy frequency, $D$ is the water depth, and $\omega$ the frequency of the wave. For a quarter daily internal wave in a hundred meter deep ocean with a buoyancy period of a quarter hour, the wave length of the wave would be $1.2 \times 10^{3}$ meters. Thus to find the horizontal wave number spectrum of waves of this frequency, an array of independent measurements must be taken within $1.2 \times 10^{3}$ meters of each other over a time short compared to the wave period. The design of this array could be optimized by creating artificial data sets with the solutions given in chapter 4 and "observing" the waves with the proposed measuring system.

However, even without measurements of the horizontal wave number spectrum of internal waves over a shelf, the solutions in chapter four allow one to make better assumptions about the internal wave climate in the coastal ocean: Friction can be assumed to be important in dissipating the waves near the coast. The internal waves forced by the deep ocean are more important over narrow shelves. The current ellipse forced by an ensemble of internal waves of a given frequency may have an ellipticity considerably less than a single wave, and the orientation of the current ellipse will be controlled primarily by the bathymetry and to a lesser extent by mean currents.

The observations analyzed in chapter five, though insufficient to determine the horizontal wave number spectrum, do allow one to make several conclusions about 
the internal wave climate in July of 1982 at the Coastal Ocean Dynamics Experiment II site. The internal wave energy at the moorings analyzed came mainly from the deep ocean, and was being dissipated as it moved onshore. Some internal wave energy must be generated on the shelf, and the internal wave energy levels are not stationary in time. Energy levels are elevated from the Garrett and Munk spectrum, but the spectral shape remains similar, though less red closer to shore. 


\subsection{Bibliography}

Beardsley, R. C., D. C. Chapman, K. H. Brink, S. R. Ramp and R. Schlitz, The Nantucket Shoals Flux Experiment (NSFE79). Part I: A Basic Description of the Current and Temperature Variability, J. Phys. Oceanogr., 15(6), 713-748, 1985.

Garrett, C. and W. Munk, Space-Time Scales of Internal Waves, Geophys. Fluid Dyn., $3,225-264,1972$.

Gill, A. E., Atmosphere-Ocean Dynamics, Academic Press, Inc., New York, 1982.

Held, I. M. and V. D. Larichev, A Scaling Theory for Horizontally Homogeneous, Baroclinically Unstable Flow on a Beta Plane, J. Atmos. Sci., 53(7), 946-952, 1995.

Mellor, G. L. and T. Yamada, Devolopment of a Turbulance Closure Model for Geophysical Fluid Problems, Rev. Geophys., 20, 851-875, 1982.

Stone, P. H., A Simplified Radiative-Dynamical Model for the Static Stability of Rotating Atmospheres, J. Atmos. Sci., 29(3), 405-418, 1972.

Visbeck, M., J. Marshall and H. Jones, Dynamics of Isolated convective Regions in the Ocean, J. Phys. Oceanogr., 26, 1721-1734, 1996. 